\title{
Theoretical and experimental constraints on large electroweak scalar multiplets
}

\author{
by
}

\author{
Kathryn A. D. Hartling
}

A thesis submitted to the

Faculty of Graduate and Postdoctoral Affairs

in partial fulfillment of the requirements

for the degree of

Doctor of Philosophy in Physics

\author{
Department of Physics \\ Carleton University \\ Ottawa-Carleton Institute of Physics \\ Ottawa, Canada
}

October 22, 2015

Copyright (C) 2015 Kathryn A. D. Hartling 


\section{Abstract}

We apply theoretical and experimental constraints to models that extend the Standard Model (SM) with scalar electroweak $S U(2)_{L}$ multiplets of isospin $T \geq 1$. Using tree-level perturbative unitarity, we determine that such multiplets may have a maximum weak isospin $T \leq 7 / 2$ (4) if they are complex (real). We also determine an upper bound on the hypercharge of a scalar $S U(2)_{L}$ multiplet as a function of its size $n=2 T+1$. Using these bounds, we study two classes of models which extend the SM by a single scalar $S U(2)_{L}$ multiplet of isospin $T \geq 2$ and which preserve an additional $U(1)$ or $Z_{2}$ symmetry. By applying bounds from precision electroweak measurements, Higgs decays to two photons, and dark matter direct-detection, we determine that the $U(1)$ models with isospin $T=5 / 2,3,7 / 2$ are excluded. The $Z_{2}$ models remain viable, but can account for at most $1 \%$ of the dark matter in the universe at masses below $1 \mathrm{TeV}$.

We also study the most general scalar potential of the Georgi-Machacek model, which extends the SM with two electroweak scalar triplets. We show that this model possesses a decoupling limit, and that in this limit the deviations of the Higgs couplings to vector bosons from their SM values may be larger and of opposite sign to those of the Two Higgs Doublet Model. We apply constraints from perturbativity, vacuum stability, electroweak precision observables, and B-physics, and demonstrate their effects on the model parameter space with numerical scans. We show that subject to these constraints - the Georgi-Machacek (GM) model can simultaneously enhance all of the Higgs couplings to Standard Model particles by up to $18 \%$, corresponding to a 39\% enhancement of all Higgs production modes. Finally, we derive general formulas for loop decays of the GM scalars to $\gamma \gamma, Z \gamma$, and $W^{+} \gamma$, and consider their effects on the scalar branching ratios in a benchmark case. 


\section{Acknowledgments}

I would like to sincerely thank all of my family, friends, and colleagues, each of whom has contributed time, knowledge, patience, and enthusiasm to this thesis. Whether at work or at play, their support and encouragement have greatly influenced my personal and professional development throughout my graduate studies.

In particular, I am truly grateful to my supervisor, Heather Logan, who is everything that I respect and admire as a physicist. I am incredibly thankful for the opportunity to learn from her wisdom and expertise, and for her unwavering support of all of my varied projects. I am further indebted to our collaborators - Kevin Earl, Kunal Kumar, Andrea Peterson, Terry Pilkington, and Marco Zaro - for their hard work and commitment to our research projects, as well as to my thesis committee, who have generously donated their time and experience to guide the progress of my thesis. I have also appreciated the help of Eva Lacelle and Joanne Martin in navigating the administrative side of my doctorate program.

A great many members of the wider physics community have also contributed to my research and professional development. I would like to thank everyone in the Carleton Theory Group for stimulating discussions, advice, and feedback. I am especially grateful to Pat Kalyniak and Steve Godfrey, who have both written many reference letters on my behalf. Thanks also to the many wonderful teachers and physicists who have guided and challenged my understanding of physics both in the classroom and at conferences throughout the years.

I am very thankful for the institutions that have financially supported my research, including Carleton University, the National Science and Engineering Research Council of Canada, and the government of Ontario. I also appreciate the efforts of 
the many laboratories and universities that have extended their hospitality to me at conferences throughout the years, particularly LPC Fermilab for their invitation to speak at the 2014 BSM Higgs Workshop.

Finally, a loud and resounding thank you to my parents, Ann and Dave, and my wonderful husband Ben for their unending love and understanding. Their support and patience have kept me going throughout the highs and lows of this undertaking, and I could not have done it without them. 


\section{Statement of Originality}

Chapters 2 and 3 of this thesis primarily review background and methods from the literature, as referenced. Chapters 4-7 contain the new results and analysis of this thesis. These chapters are based on the author's publications, as indicated in the following list. Results that were produced by collaborators are also noted.

- The results of Chp. 4 were published in Ref. [1]. All results were independently derived by both the author and collaborators.

- The results presented in Chp. 5 were published in Ref. [2].

- The results presented in Secs. 5.1-5.3.2 are the independent work of the author (although some were separately derived or verified by collaborators).

- Sec. 5.3.3 summarizes arguments based on collider constraints that were computed by collaborator Heather Logan.

- The dark matter constraints in Sec. 5.3.4 (see Figs. 5.2-5.4) were performed by collaborator Terry Pilkington; they are summarized to clarify the basis for excluding these models. However, the $h \rightarrow \gamma \gamma$ bound used in Sec. 5.3.4 (see Fig. 5.5) was applied by the author.

- The results presented in Chp. 6 were published in Ref. [3], and were presented by the author at the 2013 BNL Forum at Brookhaven National Laboratory.

- Similarly to the Chp. 5 case, the results presented in sections 6.1-6.3.3 are the work of the author, but the dark matter constraints in Sec. 6.3.5 were computed by Terry Pilkington and are summarized for completeness.

- The Sec. 7.1-7.3 results were published in Ref. [4], and were presented by the author at Pheno 2014 (Pittsburgh) and 'BSM at LPC 2014' (Fermilab). 
- The unitarity constraints in Sec. 7.2.1 have been independently derived by the author, but were first presented by Aoki and Kanemura in Ref. [5].

- The constraints from the boundedness of the potential presented in Sec. 7.2.2 were first derived by collaborator Kunal Kumar, but were independently verified by the author.

- The Sec. 7.2.3 bounds from alternative vacua were produced by collaborator Heather Logan, and are summarized for completeness.

- A bound from the oblique parameter $S$ was applied to the Georgi-Machacek (GM) model in Ref. [6], but an explicit formula was not provided. The Sec. 7.4.1 result was independently derived by the author, and is consistent with the results of Ref. [6].

- The results of Sec. 7.4 have been published in Ref. [7] and were presented by the author at 'BSM at LPC 2014' (Fermilab).

- We have adapted many results from the literature in this section, as referenced. We are the first to apply many of these results to the GM model (as noted), but these formulas are not original and have not been re-derived.

Apart from the reservations listed above, this research is entirely new and original (to our knowledge) and has not been submitted as the final project of another degree. In the appendices we present the Feynman rules of the relevant models, as well as some supplemental formulas for scalar decays used in the Georgi-Machacek model analysis. 


\section{Contents}

1 Introduction 1

2 The Standard Model and Beyond $\quad 5$

2.1 The known spectrum of elementary particles $\ldots \ldots \ldots \ldots$

2.2 The Standard Model . . . . . . . . . . . . . . . . . . . . . 7

2.2 .1 The gauge sector $\ldots \ldots \ldots \ldots \ldots$

2.2.2 The fermion sector . . . . . . . . . . . . . . 11

2.2.3 The scalar sector and the Higgs Mechanism . . . . . . . 12

2.3 Beyond the Standard Model . . . . . . . . . . . . . . . . 17

2.3.1 Standard Model difficulties and open questions _. . . . . . 17

2.3.2 BSM scalar extensions . . . . . . . . . . . . . . . 20

2.3.3 Current experimental status of scalar particles _ . . . . . 22

3 Constraints on models with scalar multiplet extensions 24

3.1 Theoretical constraints . . . . . . . . . . . . . . . 25 
3.1.1 Tree-level perturbative unitarity . . . . . . . . . . 25

3.1.2 Bounded-from-below constraints . . . . . . . . . . . . 32

3.1.3 Alternative vacua ................... 34

3.2 Experimental constraints . . . . . . . . . . . . 35

3.2.1 Direct collider searches . . . . . . . . . . . . 36

3.2.2 Constraints from the $\rho$ parameter at tree level . . . . . . . . . 39

3.2.3 Oblique parameters ................ 40

3.2.4 Loop couplings of the Higgs to photons . . . . . . . . . . . 46

3.2.5 Interactions of charged scalars with fermions . . . . . . . 52

3.2.6 Dark matter candidates . . . . . . . . . . . . . . 62

4 Constraints on the maximum isospin and hypercharge of a scalar $\begin{array}{ll}\text { electroweak multiplet } & 66\end{array}$

4.1 A model with an extra scalar multiplet . . . . . . . . . . . 67

4.2 General constraints from unitarity . . . . . . . . . . 69

4.2.1 $\mathrm{U}(1)_{Y}$ interactions .................... 69

4.2.2 $\mathrm{SU}(2)_{L}$ interactions . . . . . . . . . . . . . . 71

4.2.3 Combined electroweak gauge interactions . . . . . . . . . . . 73

5 Models with an accidental U(1) symmetry $\quad 77$

5.1 Model description . . . . . . . . . . . . . . . . . 79

5.2 Theoretical constraints . . . . . . . . . . . . . . . 80 
5.2.1 Constraints from tree-level perturbative unitarity . . . . . . . 81

5.3 Experimental constraints . . . . . . . . . . . . 85

5.3.1 Constraints from oblique parameters . . . . . . . . . 85

5.3.2 Constraints from $h \rightarrow \gamma \gamma$ decay . . . . . . . . . . . . . 87

5.3.3 Constraints from direct searches . . . . . . . . . . . 88

5.3.4 Dark matter constraints . . . . . . . . . . . . . 89

6 Models with a $\mathrm{Z}_{2}$ symmetry $\quad 95$

6.1 Model description . . . . . . . . . . . . . . . . 96

6.1.1 Particle spectrum and masses . . . . . . . . . . . . . . . 97

6.1.2 Degeneracy and clustering in the mass spectrum . . . . . . . . 100

6.1.3 Model summaries . . . . . . . . . . . . . . . . . . . 101

6.2 Theoretical constraints . . . . . . . . . . . . . . . . . 104

6.2.1 Constraints from tree-level perturbative unitarity . . . . . . . 104

6.3 Experimental constraints . . . . . . . . . . . . 106

6.3.1 Direct-detection constraints ............. 106

6.3.2 Constraints from oblique parameters . . . . . . . . . . . 106

6.3.3 Constraints from $h \rightarrow \gamma \gamma$ decay . . . . . . . . . . . . . . 112

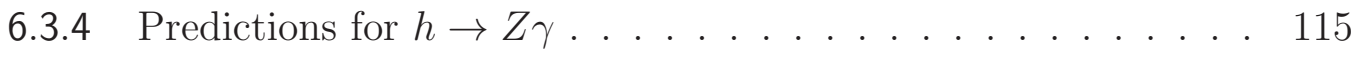

6.3.5 Dark matter constraints . . . . . . . . . . . . . . 117 
7.1 Model description . . . . . . . . . . . . . . . . . 127

7.2 Theoretical constraints . . . . . . . . . . . . . . . . . 132

7.2.1 Constraints from tree-level perturbative unitarity . . . . . . 132

7.2.2 Bounded-from-below constraints . . . . . . . . . . . . . 140

7.2.3 Avoiding alternative vacua . . . . . . . . . . . . . 148

7.3 The decoupling limit . . . . . . . . . . . . . . . . 150

7.3.1 The decoupling behaviour of the vevs and mixing angles _. . 153

7.3.2 The decoupling behaviour of the scalar masses . . . . . . . 156

7.3.3 The decoupling of tree-level Higgs couplings . . . . . . . . 157

7.3.4 The decoupling of loop-level Higgs decays _ . . . . . . . . . 161

7.4 Indirect experimental constraints . . . . . . . . . . . . . . 164

7.4.1 Constraints from oblique parameters . . . . . . . . . . . 164

7.4.2 Constraints on fermion interactions . . . . . . . . 165

7.4.3 Numerical results from theoretical and indirect experimental constraints . . . . . . . . . . . . . . 174

7.5 Constraints from direct collider searches . . . . . . . . . . . 179

7.6 Loop-decays in the Georgi-Machacek model f . . . . . . . . . . 181

7.6.1 Scalar decays to photons . . . . . . . . . . . . . . . 184

7.6.2 Scalar decays to a photon and a $Z \ldots \ldots$. . . . . 185 
7.6.3 Scalar decays to a photon and a $W \ldots \ldots$. . . . . . . . . 187

7.6.4 Decay branching ratios of the $H_{3}$ and $H_{5}$ scalars . . . . . . . . 190

8 Conclusions and Summary

A Feynman rules

A.1 $U(1)$ Models . . . . . . . . . . . . . . . . . . . . . . . . . . . . 199

A.2 $Z_{2}$ Models . . . . . . . . . . . . . . . . . . 200

A.2.1 Higgs boson couplings to scalar pairs . . . . . . . . . . . . 200

A.2.2 Gauge boson couplings to scalar pairs . . . . . . . . . . . . 201

A.3 Georgi-Machacek . . . . . . . . . . . . . . . . 204

A.3.1 Triple-scalar couplings . . . . . . . . . . . . . . . . . . 204

A.3.2 Scalar couplings to gauge bosons . . . . . . . . . . . . 207

A.3.3 Scalar couplings to fermions . . . . . . . . . . . . 209

B Scalar decays in the GM model $\quad 211$

B.1 Direct decays to $W$ and $Z$ vector bosons . . . . . . . . . . . . . . 211

B.2 Decays to off-shell vector bosons . . . . . . . . . . . . . . . . . 211

B.3 Decays to gluons . . . . . . . . . . . . . . . . . . . . . 212

B.4 Decays to one scalar and one vector boson . . . . . . . . . . . . 213

B.5 Decays to fermions . . . . . . . . . . . . . . . . . . . 214

$\begin{array}{lr}\text { References } & 214\end{array}$ 


\section{List of Tables}

2.1 Left-handed fermions and their properties . . . . . . . . . . . 7

2.2 Gauge boson properties. . . . . . . . . . . . . . . . . . . 8

2.3 Left-handed and right-handed representations. . . . . . . . . . . 11

3.1 Measurements of the signal strengths for $h \rightarrow \gamma \gamma$ and $h \rightarrow Z \gamma$ relative to the SM predictions. . . . . . . . . . . . . . . . 48

4.1 Upper limit on the hypercharge $Y$ allowed by perturbative unitarity for a complex $n$-plet of $\mathrm{SU}(2)_{L} \ldots \ldots \ldots \ldots 75$

5.1 Upper limits on $\left|\lambda_{2}\right|$ and $\left|\lambda_{3}\right|$ from unitarity, for $Y=2 T=n-1 . \quad . \quad 84$

5.2 The $95 \%$ C.L. lower limit on $\lambda_{3}$ from the $S, T$, and $U$ constraints for $5 \leq n \leq 8$ and $m_{\chi^{0}}=m_{h} / 2=62.5 \mathrm{GeV} \ldots \ldots . . . . .$.

6.1 Upper limits on $\left|\lambda_{2}\right|,\left|\lambda_{3}\right|$ and $\left|\lambda_{4}\right|$ from perturbative unitarity for the

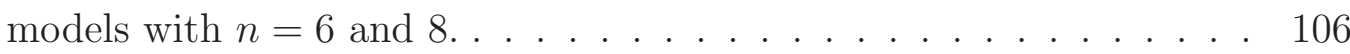

7.1 Basis states and eigenvalues of the $2 \rightarrow 2$ scalar scattering matrix in the $G M$ model. . . . . . . . . . . . . . . . ... 137 
7.2 Maximal ranges allowed to the variables used to parametrize the GM scalar potential. . . . . . . . . . . . . . . . . . . 144

7.3 Theoretical bounds on the scalar potential parameters $\lambda_{i}$ and $M_{i}$. . . 148

7.4 Values of coupling parameters for the two GM decoupling scenarios. . 152

7.5 The power law behavior of the heavy scalar masses, triplet vev, custodial singlet mixing angle, and light Higgs couplings. . . . . . . . . . . 153

7.6 Decoupling rates in the GM and 2HDM. . . . . . . . . . . . . 160

7.7 Combinations of propagating particles for the $W^{+} \gamma$ decay calculations. 190

7.8 Benchmark point for $H_{3}$ and $H_{5}$ branching ratios. . . . . . . . . . . . 191 


\section{List of Figures}

2.1 The current experimental status of Higgs decay modes. . . . . . . . . 23

3.1 Feynman diagrams contributing to $s_{1} s_{2} \rightarrow s_{3} s_{4}$ and $s s^{*} \rightarrow V_{1} V_{2}$. . . 26

3.2 Diagrams involving scalars that contribute to oblique parameters. . . 41

3.3 Diagrams contributing to $h \rightarrow V \gamma . \ldots \ldots . \ldots . \ldots . . \ldots 47$

3.4 Sample diagrams of charged scalar contributions to $R_{b}$. . . . . . . . 54

3.5 Sample diagrams of charged scalar contributions to $B_{s, d}^{0}-\bar{B}_{s, d}^{0} . \quad \ldots \quad$. . 55

3.6 Sample diagrams of charged scalar contributions to $B_{s, d}^{0} \rightarrow \mu^{+} \mu^{-}$. . $\quad 58$

3.7 Sample diagrams of charged scalar contributions to $b \rightarrow s \gamma \ldots \ldots$. . . 60

3.8 Summary of direct detection limits on the WIMP-nucleon interaction cross section. . . . . . . . . . . . . . . . . . . 64

5.1 Constraints from oblique parameters $S, T$ and $U$ in the $\mathrm{U}(1)$ model. . $\quad 86$

5.2 The fractional relic density for $m_{\chi^{0}}$ in the $U(1) n=6$ model. . . . . . 91

5.3 The same as Fig. 5.2 but for $n=7$ (left) and 8 (right). . . . . . . . . 91

5.4 Fractional relic density as a function of $\Lambda_{0}$ for $m_{\chi^{0}} \simeq m_{h} / 2 \ldots \ldots$. . . 93 
5.5 The $h \rightarrow \gamma \gamma$ partial width as a function of $\Lambda_{0}$ for $m_{\chi^{0}}=m_{h} / 2$. . .

6.1 Sample mass spectra for the $Z_{2} n=6$ model as a function of $\lambda_{4}$. . . 101

6.2 Values of $\lambda_{3}$ and $\lambda_{4}$ allowed at 95\% C.L. by $S, T, U$ in the $Z_{2}$ model. 109

6.3 Unitarity-allowed $U$ and $T$ values in the $Z_{2}$ model. . . . . . . . . . . 110

6.4 Unitarity-allowed $S$ and $T$ values in the $Z_{2}$ model. . . . . . . . . . . . 111

6.5 Unitarity-allowed $U$ and $S$ values in the $Z_{2}$ model. . . . . . . . . . . . 112

6.6 Allowed range for the first mass splitting as a function of $m_{\zeta^{0, r}}$. . . 113

6.7 Allowed range for a larger mass splitting as a function of $m_{\zeta^{0, r}} \ldots \ldots$. . 114

6.8 Allowed range of the $Z_{2}$ model $\lambda_{2}$ as a function of $m_{\zeta^{0, r}} \ldots \ldots \ldots$

6.9 Allowed range of $R_{Z \gamma}$ versus $R_{\gamma \gamma}$ in the $Z_{2}$ model. . . . . . . . . 116

6.10 The $Z_{2}$ relic density fraction as a function of $m_{\zeta^{0, r}}$. . . . . . . . . 118

6.11 Predictions for the $Z_{2}$ direct detection cross section as a function of the mass of the dark matter candidate $m_{\zeta^{0, r}} \ldots \ldots$. . . . . . . . . . . 120

7.1 Constraints on the $\left(\lambda_{3}, \lambda_{4}\right)$ and $\left(\lambda_{5}, \lambda_{2}\right)$ planes from unitarity. . . . . . 140

7.2 The boundary of the GM parameter space in the $(\zeta, \omega)$ plane. . . . . 146

7.3 Bounds on the $\left(\lambda_{3}, \lambda_{4}\right)$ plane from unitarity and BFB constraints. . . 147

7.4 Decoupling of the GM vacuum expectation values. . . . . . . . . . . . 154

7.5 Decoupling of the GM mixing angles. . . . . . . . . . . . . . 156

7.6 Decoupling of GM masses and mass splittings. . . . . . . . . . . . . 157

7.7 Decoupling of GM Higgs couplings to fermions and gauge bosons. . . 158 
7.8 Numerical scan of Higgs couplings to fermions and gauge bosons. . . 159

7.9 Decoupling of GM Higgs couplings to $\gamma \gamma \ldots \ldots$. . . . . . . . . . . . . 162

7.10 Decoupling of GM Higgs couplings to $Z \gamma$. . . . . . . . . . . . . 163

7.11 Constraints on $m_{3}$ and $v_{\chi}$ in the GM model from $R_{b}, B_{s}^{0}-\bar{B}_{s}^{0}$ mixing, $\overline{\mathrm{BR}}\left(B_{s}^{0} \rightarrow \mu^{+} \mu^{-}\right)$, and $\mathrm{BR}(b \rightarrow s \gamma) . \ldots \ldots . \ldots . . \ldots 167$

7.12 Effect of the experimental bounds on $v_{\chi}$ in the GM model. . . . . . . 175

7.13 Effect of the experimental bounds on $S$ and $m_{3}$ as a function of $m_{5}$. . 176

7.14 Effect of the experimental bounds on the GM Higgs couplings. . . . . 177

7.15 Correlations between $\kappa_{V}$ and $\kappa_{f}$ in the GM model. . . . . . . . . . . 178

$7.16 \kappa_{V}$ as a function of the mass of the lightest new scalar. . . . . . . . 179

7.17 Diagrams contributing to $h \rightarrow V \gamma . \ldots \ldots . \ldots . \ldots 183$

7.18 Branching ratios of the $H_{3}^{0}, H_{3}^{+}, H_{5}^{0}$ and $H_{5}^{+}$. . . . . . . . . . . 192 


\section{Chapter 1}

\section{Introduction}

The Standard Model (SM) of particle physics provides a very successful description of the interactions and phenomenology of all experimentally discovered elementary particles. In particular, the predictions of the SM electroweak theory have been tested to a very high precision at many experiments, including high-energy electron-positron colliders such as Large Electron-Positron (LEP) collider and the Stanford Linear Collider (SLC). Furthermore, in 2012 a scalar particle consistent with a Higgs boson - the last SM particle charged under the electroweak symmetry - was discovered at the Large Hadron Collider (LHC). The Higgs boson is responsible for breaking the $S U(2)_{L} \times U(1)$ electroweak symmetry, a mechanism that generates mass for the other elementary particles. To date, the measured properties of this newly discovered scalar are also in good agreement with the predictions of the SM.

Despite the success of the SM, there still remain many open questions. For example, this theory does not account for the experimental observations of neutrino masses and dark matter. These unexplained phenomena have prompted the development of many beyond-the-SM (BSM) theories that include additional $S U(2)_{L}$ scalar 
multiplets under the electroweak symmetry. These extra scalar particles may provide dark matter candidates or participate in electroweak symmetry breaking (EWSB) as additional Higgs bosons. Most of these models are minimalist in their extensions, introducing only a new electroweak singlet or doublet (multiplets of size $n=1,2$ ); examples of these include the Two Higgs Doublet Model (2HDM) [8], the Minimal Supersymmetric Standard Model (MSSM) [9-11], the Inert Doublet model [12], and singlet dark matter [13].

However, multiplets larger than doublets $(n \geq 3)$ have become more popular in recent years, as they present certain advantages when it comes to model building. Models with a scalar electroweak multiplet of isospin $T \geq 2$ often have an accidental symmetry in the scalar potential that leads to a dark matter candidate [14-16]. Larger representations in $S U(2)_{L}$ also allow for higher-dimension operators that are helpful in models of neutrino mass generation [17-29]. Higgs models where the SM Higgs doublet mixes with a large $S U(2)_{L}$ scalar multiplet have also been considered [3033]; these models have the ability to enhance the couplings of the SM-like Higgs to other SM particles, unlike Higgs models with only singlets and doublets. Such an enhanced-coupling mechanism could have important implications for Higgs physics, as the corresponding enhancement to Higgs production modes could mask BSM effects at the LHC [34].

As such, models which extend the SM with large scalar electroweak multiplets are able to account for a very wide range of phenomenology, which may be limited by considering a variety of constraints. Some bounds arise from theoretical considerations, such as the requirement that perturbative unitarity be preserved at tree level, or that the scalar potential have an appropriate global minimum in order to achieve EWSB. Others arise from experimental measurements and searches for BSM 
physics. A variety of electroweak precision observables (e.g. $\rho$, the oblique parameters, $R_{b}$ ), Higgs decays (e.g. $h \rightarrow \gamma \gamma$ ), and B-physics processes (e.g. $B_{s}^{0}-\bar{B}_{s}^{0}$ meson mixing, $B_{s}^{0} \rightarrow \mu^{+} \mu^{-}$, and $b \rightarrow s \gamma$ ) will be altered at loop-level by electroweak scalar multiplets. As these observables have been measured at a variety of collider experiments (e.g. LEP, LHC, CLEO, Belle, BaBar), deviations in the model predictions from the measurements of these processes can be used to constrain the properties of BSM scalars. Models which include a dark matter candidate are further subject to limits from the observed relic abundance of dark matter, as well as constraints from dark matter direct-detection experiments [35]. Limits from theoretical, indirect, and dark matter direct-detection constraints can be combined with those from direct searches at colliders to significantly constrain the model parameter space.

Throughout this thesis, we apply these constraints to several models which extend the SM with a large scalar electroweak multiplet, and investigate the phenomenology of the viable parameter space. We begin by determining the maximal values of isospin $T$ and hypercharge $Y$ that an electroweak scalar multiplet may have based on tree-level constraints from perturbative unitarity. We then use these bounds to consider the limits upon inert scalar electroweak multiplets that do not participate in the Higgs mechanism. We investigate two general classes of models with scalar multiplets of isospin $T \geq 2(n \geq 5)$ that respectively preserve an additional $U(1)$ or $Z_{2}$ symmetry in the scalar potential. We apply a series of theoretical and experimental constraints to each of these models, including bounds from tree-level perturbative unitarity, the oblique parameters, $h \rightarrow \gamma \gamma$, and dark matter direct-detection limits. After determining the allowed parameter space of each model, we discuss their viability as a description of dark matter.

We then consider a Higgs model with a large scalar multiplet. The Georgi- 
Machacek (GM) model [30] extends the SM by adding two electroweak triplets to the scalar sector, both of which mix with the SM Higgs doublet. However, this model can also be generalized to quartets, quintets, or sextets [36], can be incorporated into models of Little Higgs [37,38] or Supersymmetry [39-41], and can accommodate the seesaw mechanism of neutrino mass generation [42]. Due to this range of applications, the GM model provides a valuable benchmark for investigations of the enhancement of Higgs couplings and their ability to hide the effects of BSM physics at the LHC.

In our analysis of the GM model, we begin by constraining the parameters of the scalar potential using requirements of tree-level perturbative unitarity and vacuum stability. Under these theoretical constraints, we consider the behaviour of the couplings, masses, and mixing angles in the decoupling limit of the model. We then apply a series of indirect experimental bounds from electroweak precision observables and B-physics processes. We illustrate the effects of these constraints by performing a numerical scan of the parameter space, and consider the ability of the model to enhance the couplings of the SM-like Higgs. Finally, we derive the formulas for loop-decays of the GM scalars to $\gamma \gamma, Z \gamma$, and $W \gamma$, and consider the effect of these decay modes on the branching ratios in a benchmark case.

This thesis is outlined as follows. In Chp. 2 we review the SM and discuss some of the motivations for considering BSM scalar extensions. Chapter 3 outlines the general theoretical and experimental constraints that may be applied to models with scalar electroweak multiplets. In Chp. 4 we determine the maximal values of isospin $T$ and hypercharge $Y$ for a scalar $S U(2)_{L}$ multiplet. In Chps. 5, 6, and 7 we consider the limits and phenomenology of the $U(1), Z_{2}$, and GM models respectively. Finally, we summarize our conclusions in Chp. 8. The Feynman rules and some scalar decay formulas for the various models may be found in the appendices. 


\section{Chapter 2}

\section{The Standard Model and Beyond}

\subsection{The known spectrum of elementary particles}

To date, particle physics experiments have discovered 17 types of particles which are believed to be elementary. These particles are categorized by their mass, electric charge, and quantum spin (see Tables 2.1-2.2 for summaries of the particle properties). For each particle of non-zero electric charge there is also an anti-particle which has opposite electric charge; neutral particles generally function as their own anti-particle. ${ }^{1}$

Twelve of the known elementary particles fall into the category of fermions, or particles with half-integer spin. ${ }^{2}$ These fermions are the building-blocks of matter, as they may bind to form composite particles and atoms. Six of the fermions are

\footnotetext{
${ }^{1}$ It is currently unknown whether the observed neutrinos are in fact their own anti-particles (i.e. whether they are Majorana or Dirac neutrinos). However, a discussion of this issue is beyond the scope of this thesis.

${ }^{2}$ Spin is an intrinsic quantum number that is mathematically similar to the classical concept of angular momentum. Fermions (1/2-integer spin) obey Fermi-Dirac statistics and the Pauli Exclusion Principle.
} 
leptons, which are divided into three classes called generations (see Table 2.1). Each generation contains a lepton — the electron $e$, the muon $\mu$, or the tau $\tau$ - with electric charge $^{3} Q=-1$ and a corresponding neutrino of charge $Q=0\left(\nu_{e}, \nu_{\mu}\right.$, $\left.\nu_{\tau}\right)$. The masses of the charged leptons increase with generation number, while the neutrinos have tiny masses that are negligible for our purposes [43].

There are also six quarks - up $(u)$, down $(d)$, charm $(c)$, strange $(s)$, top $(t)$, and bottom $(b)$ — which are similarly paired into three generations by charge and mass. The up, charm, and top quarks all have charge $Q=2 / 3$ (and thus are frequently referred-to as 'up-type' quarks), while the down, strange, and bottom quarks have $Q=-1 / 3$ ('down-type' quarks). Unlike leptons, quarks are never found alone in nature; they are always bound to other quarks in configurations that yield a total integer electric charge.

These matter particles are known to interact through the electromagnetic, weak, and strong forces. Such interactions occur through the exchange of a vector (spin 1) gauge boson ${ }^{4}$ associated with the force. To date, experiments have discovered four vector bosons: photons carry the electromagnetic force, $W$ and $Z$ bosons carry the weak force, and gluons carry the strong force. Only the $W$ bosons carry electric charge $(Q=1)$, while the other gauge bosons are neutral. The weak bosons $W$ and $Z$ are massive, while gluons and photons are massless (see Table 2.2).

Finally, a scalar (spin-0) particle has also been discovered, and is known as the Higgs boson. It is believed that the mass of any fundamental particle is determined by its interactions with the Higgs. The Higgs is electrically neutral $(Q=0)$ and has a mass of $125 \mathrm{GeV}$ (about 133 times the mass of a proton) [44].

\footnotetext{
${ }^{3}$ In units of the magnitude of the charge of the electron.

${ }^{4}$ The term boson refers to any particle with integer spin. Bosons obey Bose-Einstein statistics.
} 
Table 2.1: Left-handed fermions and their properties (see Sec. 2.2.2 for right-handed).

\begin{tabular}{|c|c|c|c|c|c|c|c|}
\hline Type & Generation & Name & Symbol & Mass $(\mathrm{MeV})$ [43] & $Q$ & $Y$ & $T^{3}$ \\
\hline \multirow{6}{*}{ Leptons } & \multirow[t]{2}{*}{1} & $\begin{array}{l}\text { Electron } \\
\text { neutrino }\end{array}$ & $\nu_{e}$ & $\leq 2 \times 10^{-6}$ & 0 & -1 & $1 / 2$ \\
\hline & & Electron & $e$ & $\begin{array}{l}0.510998928 \\
\pm 1.1 \times 10^{-8}\end{array}$ & -1 & -1 & $-1 / 2$ \\
\hline & \multirow[t]{2}{*}{2} & $\begin{array}{c}\text { Muon } \\
\text { neutrino }\end{array}$ & $\nu_{\mu}$ & $\leq 2 \times 10^{-6}$ & 0 & -1 & $1 / 2$ \\
\hline & & Muon & $\mu$ & $\begin{array}{l}105.6583715 \\
\pm 3.5 \times 10^{-6}\end{array}$ & -1 & -1 & $-1 / 2$ \\
\hline & \multirow[t]{2}{*}{3} & $\begin{array}{c}\text { Tau } \\
\text { neutrino }\end{array}$ & $\nu_{\tau}$ & $\leq 2 \times 10^{-6}$ & 0 & -1 & $1 / 2$ \\
\hline & & Tau & $\tau$ & $1776.82 \pm 0.16$ & -1 & -1 & $-1 / 2$ \\
\hline \multirow{6}{*}{ Quarks } & \multirow{2}{*}{1} & Up & $u$ & $2.3 \pm 0.7$ & $2 / 3$ & $1 / 3$ & $1 / 2$ \\
\hline & & Down & $d$ & $4.8 \pm 0.5$ & $-1 / 3$ & $1 / 3$ & $-1 / 2$ \\
\hline & \multirow[t]{2}{*}{2} & Charm & $c$ & $\begin{array}{c}1.275 \times 10^{3} \\
\pm 0.075 \times 10^{3} \\
\end{array}$ & $2 / 3$ & $1 / 3$ & $1 / 2$ \\
\hline & & Strange & $s$ & $95 \pm 5$ & $-1 / 3$ & $1 / 3$ & $-1 / 2$ \\
\hline & \multirow[t]{2}{*}{3} & $\begin{array}{c}\text { Top } \\
\text { quark }\end{array}$ & $t$ & $\begin{array}{l}173.21 \times 10^{3} \\
\pm 0.87 \times 10^{3}\end{array}$ & $2 / 3$ & $1 / 3$ & $1 / 2$ \\
\hline & & Bottom & $b$ & $\begin{array}{l}4.18 \times 10^{3} \\
\pm 3 \times 10^{3}\end{array}$ & $-1 / 3$ & $1 / 3$ & $-1 / 2$ \\
\hline
\end{tabular}

\subsection{The Standard Model}

The Standard Model (SM) of particle physics is the most complete theory to date that describes the fundamental interactions and behaviour of the known elementary particles $[43,45]$. It is an effective quantum field theory, meaning that it describes the subatomic world in terms of fields. These fields are quantized, and their quanta correspond to elementary particles. The theory is an effective field theory (EFT) in 
Table 2.2: Gauge boson properties.

\begin{tabular}{|c|c|c|c|c|c|}
\hline Force & Name & Field Symbol & Mass $(\mathrm{GeV})[43]$ & $Q$ & Colored? \\
\hline \hline Electromagnetic & Photon & $A_{\mu}$ & 0 & 0 & No \\
\hline \multirow{2}{*}{ Weak } & $\mathrm{Z}$ & $Z_{\mu}$ & $91.1876 \pm 0.0021$ & 0 & No \\
\cline { 2 - 6 } & $\mathrm{W}$ & $W_{\mu}^{ \pm}$ & $80.385 \pm 0.015$ & \pm 1 & No \\
\hline \multirow{2}{*}{ Strong } & Gluon & $G_{\mu}$ & 0 & 0 & Yes \\
\hline
\end{tabular}

that it is accurate only up to some cut-off energy scale, and describes only the physics that is relevant over this energy range. Above the cut-off scale the SM is expected to break down due to the emergence of physics that is not included within the theory. It is possible that the SM is valid all the way up to the Planck scale $\left(M_{\text {Planck }} \approx 10^{19}\right.$ $\mathrm{GeV}$ ), where gravity becomes relevant ${ }^{5}$ and classical physics begins to dominate over quantum effects. However, there are many compelling reasons to believe that the SM cutoff is in fact much lower $(\approx 1 \mathrm{TeV})$, and that some form of new physics - as yet undetected - manifests at this scale (as will be further discussed in Sec. 2.3.1).

\subsubsection{The gauge sector}

The SM is also a non-abelian gauge theory, meaning that the Lagrangian which describes the interactions of the subatomic particles is locally gauge invariant ${ }^{6}$ under a certain symmetry group, $S U(3)_{c} \times S U(2)_{L} \times U(1)_{Y}[45,46]$. It is non-abelian because the generators of the $S U(3)$ and $S U(2)$ sectors are non-commutative. The

\footnotetext{
${ }^{5}$ The SM does not include gravity. Gravity is an extremely weak force and is negligible at the relevant scales for particle interactions.

${ }^{6}$ The Lagrangian is invariant under local gauge transformations of the form $\phi \rightarrow U(x) \phi$ for matter fields $\phi$ (spin 0 or $\pm \frac{1}{2}$ ) and $\tau^{a} A_{\mu}^{a} \rightarrow U(x) \tau^{a} A_{\mu}^{a} U^{\dagger}(x)-\frac{i}{g}\left(\partial_{\mu} U(x)\right) U^{\dagger}(x)$ for gauge fields $A_{\mu}^{a}$ (spin 1) respectively [46-48]. Here $U(x)$ is an element of the symmetry group of the Lagrangian, $\tau^{a}$ are the generators of the symmetry group, and $g$ is the gauge coupling.
} 
properties of an elementary particle are determined by the quantum numbers that define how it transforms under this symmetry. For each generator of the group there is a corresponding gauge field, the quanta of which correspond to the force-carrying vector gauge bosons.

The $S U(3)_{c}$ symmetry describes the strong force sector, and its generators correspond to gluons. As $S U(3)$ has eight generators, there are eight types of gluons that are distinguished by the quantum number called color. There are three colors red, green, and blue — and gluons are identified by the eight possible combinations of a color and an anti-color (i.e. red-antigreen or green-antiblue). This symmetry remains unbroken after the introduction of the Higgs field, and as a result gluons do not interact with the Higgs boson and remain massless. This strong sector of the SM is described by an EFT within the SM known as Quantum Chromodynamics (QCD) [49].

The $S U(2)_{L} \times U(1)_{Y}$ symmetries together describe the electromagnetic and weak (electroweak) interactions in the SM. The quantum number conserved by the $S U(2)_{L}$ symmetry is known as weak isospin ${ }^{7}$, while the $U(1)_{Y}$ symmetry conserves hypercharge. However, these symmetry groups cannot be treated distinctly, as this electroweak symmetry is spontaneously broken by the introduction of a Higgs field. This is known as electroweak symmetry breaking (EWSB), and the Higgs mechanism which causes it is responsible for the generation of mass (further details in Sec. 2.2.3).

Before EWSB there are four gauge fields corresponding to the combined $3+1$ generators of the $S U(2)$ and $U(1)$ symmetries: $W_{\mu}^{1,2,3}$ are the gauge fields of weak isospin $S U(2)_{L}$ and $B_{\mu}$ is the gauge field of hypercharge $U(1)_{Y}$. However, after EWSB only a $U(1)_{\text {EM }}$ symmetry is conserved. This symmetry is associated with the elec-

\footnotetext{
${ }^{7}$ Not to be confused with intrinsic quantum spin, or strong isospin (isobaric spin).
} 
tromagnetic force, and is described by an EFT known as Quantum Electrodynamics (QED). It conserves electric charge and the gauge field associated with its single generator corresponds to the photon. This photon is a linear combination of the $S U(2)_{L} \times U(1)_{Y}$ gauge fields $W_{\mu}^{3}$ and $B_{\mu}$,

$$
A_{\mu}=\sin \theta_{W} W_{\mu}^{3}+\cos \theta_{W} B_{\mu}
$$

while the electric charge $Q=T^{3}+Y / 2$ is similarly a linear combination of hypercharge $(Y)$ and the third component of weak isospin $\left(T^{3}\right)$. The remaining three linear combinations of the original fields correspond to the $W^{+}, W^{-}, 8$ and $Z$ gauge bosons of the weak force,

$$
\begin{aligned}
W_{\mu}^{ \pm} & =\frac{1}{\sqrt{2}}\left(W_{\mu}^{1} \mp i W_{\mu}^{2}\right), \\
Z_{\mu} & =\cos \theta_{W} W_{\mu}^{3}-\sin \theta_{W} B_{\mu} .
\end{aligned}
$$

The rotation angle $\theta_{W}$ of these linear combinations is known as the Weinberg angle, and has an empirical value of $\theta_{W}=28.75$ degrees [43].

The covariant derivative associated with these gauge fields determines how they interact with scalars $\phi$ and fermion spinors $\psi$ through Lagrangian terms including factors of $\mathcal{D}_{\mu} \phi$ and $\mathcal{D}_{\mu} \psi$. The covariant derivative $\mathcal{D}_{\mu}$ can be equivalently written in different field bases as

$$
\begin{aligned}
\mathcal{D}_{\mu} & =\partial_{\mu}-i g T^{a} W_{\mu}^{a}-i \frac{g^{\prime}}{2} Y B_{\mu}, \\
& =\partial_{\mu}-i \frac{g}{\sqrt{2}}\left(W_{\mu}^{+} T^{+}+W_{\mu}^{-} T^{-}\right)-i g W_{\mu}^{3} T^{3}-i g^{\prime} B_{\mu} \frac{Y}{2}, \\
& =\partial_{\mu}-i \frac{g}{\sqrt{2}}\left(W_{\mu}^{+} T^{+}+W_{\mu}^{-} T^{-}\right)-i \frac{e}{s_{W} c_{W}} Z_{\mu}\left(T^{3}-s_{W}^{2} Q\right)-i e A_{\mu} Q,
\end{aligned}
$$

where $a=1,2,3, T^{a}$ are the generators for $S U(2), e$ is the electric coupling strength of the photon, $c_{W}=\cos \theta_{W}, s_{W}=\sin \theta_{W}$, and $g$ and $g^{\prime}$ are gauge coupling constants

\footnotetext{
${ }^{8} W^{-}$is the anti-particle of the positively charged $W^{+}$.
} 
Table 2.3: Left-handed and right-handed representations.

\begin{tabular}{|c|c|c|}
\hline & Left-handed & Right-handed \\
\hline \hline Leptons & $L_{L}=\left(\nu_{\ell}, \ell_{L}\right)^{T}$ & $\ell_{R}$ \\
\hline Quarks & $Q_{L}=\left(u_{L}, d_{L}\right)^{T}$ & $u_{R}, d_{R}$ \\
\hline$T^{3}$ & $(1 / 2,-1 / 2)^{T}$ & 0 \\
\hline
\end{tabular}

for $S U(2)$ and $U(1)_{Y}$ respectively. The hypercharge operator $Y$ is defined in the convention that the electric charge is $Q=T^{3}+Y / 2$. The gauge couplings $g$ and $g^{\prime}$ are related to $e$ and the Weinberg angle $\theta_{W}$ through $e=g \sin \theta_{W}=g^{\prime} \cos \theta_{W}$, and the generators $T^{ \pm}$are related to the $S U(2)$ generators through

$$
T^{ \pm}=T^{1} \pm i T^{2}
$$

\subsubsection{The fermion sector}

Leptons and quarks are placed in representations of the SM symmetry group. As quarks interact via the strong force, they are placed in 3 representations of the $S U(3)_{c}$ color group, indicating that they are color-charged; they may be a red, green, or blue particle. The fermion fields $\Psi$ may be polarized into states of left or right-handed chirality according to

$$
\Psi_{L, R}=P_{L, R} \Psi, \quad \text { where } \quad P_{L, R}=\left(1 \mp \gamma_{5}\right) / 2
$$

Because the weak force is known to differentiate between fermions of different chirality, right- and left-handed fermions are placed in different representations of the $S U(2)_{L} \times U(1)_{Y}$ symmetry. As the weak force operates only on left-handed 
fermions, these are placed in $S U(2)$ doublets. ${ }^{9}$ These doublets correspond to the fermion pairs found in each generation. The upper component has $T^{3}=1 / 2$ and the lower component has $T^{3}=-1 / 2$. An appropriate choice of hypercharge $Y$ ( -1 for leptons and $1 / 3$ for quarks) then leads to the correct electric charge $Q=T^{3}+Y / 2$ for both fermions in each doublet. In contrast, right-handed fermions are placed in singlets of $S U(2)$, with $T^{3}=0$ and $Y=2 Q$. The SM includes right-handed leptons and quarks, but no right-handed neutrinos.

\subsubsection{The scalar sector and the Higgs Mechanism}

In the SM, the Higgs field is embedded in a scalar doublet of $S U(2)_{L}$ with hypercharge $Y=1$,

$$
\Phi=\left(\begin{array}{c}
\phi^{+} \\
\phi^{0}
\end{array}\right)=\frac{1}{\sqrt{2}}\left(\begin{array}{c}
\phi_{1}+i \phi_{2} \\
\phi_{3}+i \phi_{4}
\end{array}\right),
$$

where $\phi^{+}$is a charged complex scalar, $\phi^{0}$ is a neutral complex scalar, and $\phi_{1,2,3,4}$ are real scalar degrees of freedom. The most general renormalizable and $\mathrm{SU}(2)_{L}$ invariant scalar Lagrangian is

$$
\mathcal{L}_{\text {scalar }}=\left|\mathcal{D}_{\mu} \Phi\right|^{2}-V(\Phi)=\left|\mathcal{D}_{\mu} \Phi\right|^{2}-\mu^{2}|\Phi|^{2}-\lambda|\Phi|^{4},
$$

where $V(\Phi)$ is a potential energy term that describes the self-interactions of $\Phi$, and the covariant derivative $D_{\mu}$ is given by Eq. (2.4).

The scalar potential $V(\Phi)$ will have a minimum value at

$$
\left\langle\Phi^{\dagger} \Phi\right\rangle=\frac{-\mu^{2}}{2 \lambda}=\frac{v^{2}}{2}
$$

when $-\mu^{2}>0$. As this defines only the expectation value of $\Phi^{\dagger} \Phi$, we are free to choose the expectation value of $\Phi$ to be

\footnotetext{
${ }^{9}$ This is why the $S U(2)$ is commonly subscripted by $L$.
} 


$$
\langle\Phi\rangle=\frac{1}{\sqrt{2}}\left(\begin{array}{l}
0 \\
v
\end{array}\right)
$$

which corresponds to $\left\langle\phi_{1,2,4}\right\rangle=0$ and $\left\langle\phi_{3}\right\rangle=v$. We may then rewrite $\phi_{3}=h+v$. When inserted into the scalar potential of Eq. (2.10), the vacuum expectation value (vev) $v$ leads to the following terms

$$
V(\Phi) \supset v^{2} \lambda h^{2}+\lambda v h^{3}+\frac{\lambda}{4} h^{4}
$$

where we have used Eq. (2.11) to replace $\mu$. The first of these terms is a mass term for $h, m_{h}^{2}=2 v^{2} \lambda$. As we will see below, the vev will similarly generate mass terms of form $\frac{1}{2} m^{2} V_{\mu} V^{\mu}$ for the weak gauge fields. As these terms are not gauge invariant, the vev has spontaneously broken the electroweak symmetry. However, as no mass term is generated for the photon the Lagrangian preserves electric charge symmetry, such that $S U(2)_{L} \times U(1) \rightarrow U(1)_{\mathrm{EM}}$.

This $3+1 \rightarrow 1$ symmetry-breaking pattern must generate three massless particles according to Goldstone's theorem: "for every spontaneously broken continuous symmetry, the theory must contain a massless particle $[48,50,51] . "$ These Goldstone bosons correspond to $\phi_{1}, \phi_{2}$, and $\phi_{4}$. With an appropriate gauge choice - known as unitarity gauge - the $\phi_{1,2,4}$ fields may be rotated away so that they disappear from the Higgs doublet. Their degrees of freedom are 'eaten' by the weak gauge bosons $W^{ \pm}$and $Z$; they become longitudinal polarizations for these bosons, causing them to become massive. We may therefore write the scalar doublet in the unitary gauge as

$$
\Phi=\frac{1}{\sqrt{2}}\left(\begin{array}{c}
0 \\
h+v
\end{array}\right)
$$

where $h$ is a scalar field called the Higgs boson.

The terms involving $W_{\mu}^{a}$ and $B_{\mu}$ in the covariant derivative result in the fol- 
lowing interaction terms between the scalar $h$ and the vector bosons,

$$
\begin{aligned}
& \left|\mathcal{D}_{\mu} \Phi\right|^{2}=\left|\partial_{\mu} \Phi-i \frac{g}{2} \sigma^{a} W_{\mu}^{a} \Phi-i \frac{g^{\prime}}{2} Y B_{\mu} \Phi\right|^{2} \\
& =\frac{1}{2} \partial_{\mu} h \partial^{\mu} h+\frac{1}{8}(v+h)^{2}\left[g^{2}\left|W_{\mu}^{1}-i W_{\mu}^{2}\right|^{2}+\left(-g W_{\mu}^{3}+g^{\prime} B_{\mu}\right)^{2}\right] \\
& \supset \frac{v^{2}}{8}\left[\left(\begin{array}{ll}
W_{\mu}^{-} & W_{\mu}^{+}
\end{array}\right)\left(\begin{array}{cc}
g^{2} & 0 \\
0 & g^{2}
\end{array}\right)\left(\begin{array}{c}
W_{\mu}^{+} \\
W_{\mu}^{-}
\end{array}\right)+\left(\begin{array}{cc}
W_{\mu}^{3} & B_{\mu}
\end{array}\right)\left(\begin{array}{cc}
g^{2} & -g g^{\prime} \\
-g g^{\prime} & g^{\prime 2}
\end{array}\right)\left(\begin{array}{c}
W_{\mu}^{3} \\
B_{\mu}
\end{array}\right)\right],
\end{aligned}
$$

where $T^{a}=\sigma^{a} / 2\left(\sigma^{a}\right.$ are the Pauli matrices $)$. We have also used Eq. (2.2) and the fact that $Y=1$ for the Higgs doublet $\Phi$.

Eq. (2.17) demonstrates that the vev $v$ of the Higgs field leads to mass terms of form $\frac{1}{2} m^{2} V_{\mu} V^{\mu}$ for the gauge fields. The couplings for these terms form mass matrices, which may be diagonalized to identify the mass states that one would observe in nature. One will note that $W_{\mu}^{ \pm}$are mass states, as their mass matrix is diagonal. However, $W_{\mu}^{3}$ and $B_{\mu}$ are not, as indicated by the off-diagonal terms in the second mass matrix; these correspond to mixed mass terms of form $c B_{\mu} W^{3 \mu}$ in the Lagrangian. By diagonalizing this second mass matrix we obtain the physical mass states $Z_{\mu}$ and $A_{\mu}$ as defined by Eqs. (2.1) and (2.3). Eq. (2.16) then reduces to

$$
\left|\mathcal{D}_{\mu} \Phi\right|^{2}=\frac{1}{2} \partial_{\mu} h \partial^{\mu} h+\frac{1}{8}\left(v^{2}+2 v h+h^{2}\right)\left[2 g^{2} W_{\mu}^{+} W^{\mu-}+\left(g^{2}+g^{\prime 2}\right) Z_{\mu} Z^{\mu}\right]
$$

where we have defined

$$
\cos \theta_{W}=\frac{g}{\sqrt{g^{2}+g^{\prime 2}}} \quad \text { and } \quad \sin \theta_{W}=\frac{g^{\prime}}{\sqrt{g^{2}+g^{\prime 2}}} .
$$

The masses of the bosons may be read off the Lagrangian in Eq. (2.18) as their squares are merely the mass term couplings. ${ }^{10}$ We therefore obtain

$$
m_{W}^{2}=\frac{g^{2} v^{2}}{4}, \quad m_{Z}^{2}=\frac{v^{2}}{4}\left(g^{2}+g^{\prime 2}\right)=\frac{g^{2} v^{2}}{4 \cos ^{2} \theta_{W}}, \quad M_{A}^{2}=0 .
$$

\footnotetext{
${ }^{10}$ Recall that when reading couplings from Lagrangian terms we multiply by $i$ and a symmetry factor to account for all possible permutations of identical fields; for example, if we have a term $c Z^{\mu} Z_{\nu}$ the Feynman rule is $2 i c g_{\mu \nu}$ as the two $Z_{\mu}$ fields are interchangeable.
} 
The $W_{\mu}^{ \pm}$and $Z_{\mu}$ bosons gain mass terms due to the non-zero Higgs vev $v$. As the photon does not couple to the Higgs it remains massless, as does the gluon, indicating that the electromagnetic $U(1)_{\mathrm{EM}}$ and strong $S U(3)_{c}$ gauge symmetries are preserved. Note that the experimentally measured mass $m_{W}$ of the $W$ boson and the sine of the Weinberg angle $\sin \theta_{W}$ may be used to set the gauge couplings $g$ and $g^{\prime}$.

The relationship between the $W$ and $Z$ gauge boson masses $m_{W} \simeq m_{Z} c_{W}$ is an important feature of the SM, as it has been experimentally tested to very high precision. This relationship arises from an additional approximate symmetry in the SM known as custodial symmetry. As seen in Eq. (2.10), the SM scalar potential preserves a global $S U(2)_{L} \times S U(2)_{R}$, as it only involves terms with $\left|\Phi^{\dagger} \Phi\right|^{2}$. However, the $S U(2)_{R}$ symmetry is weakly broken by the gauging of hypercharge, and so this symmetry is only approximate. After EWSB, the $S U(2)_{L} \times S U(2)_{R}$ symmetry is broken to $S U(2)_{c}$, its diagonal part. This $S U(2)_{c}$ custodial symmetry preserves an approximate rotation symmetry between electroweak gauge bosons $W^{1} \leftrightarrow W^{2} \leftrightarrow W^{3}$; note in Eqs. (2.17-2.20) that if $g^{\prime}=0\left(c_{W}=1\right)$ then $m_{W}=m_{Z}$. When this symmetry is weakly broken by the gauging of hypercharge $\left(g^{\prime} \neq 0\right)$, it requires the familiar relationship $m_{W} \simeq m_{Z} c_{W}$ between the electroweak gauge boson masses.

Eq. (2.18) also allows us to read off the couplings of two $W$ or $Z$ bosons to one or two Higgs fields $h$. These $V_{\mu} V_{\nu} h^{n}$ couplings - where $n=1,2$ is the number of Higgs bosons and $V=W^{ \pm}, Z$ - have the form $i e^{2} C_{V V h^{n}} g_{\mu \nu}{ }^{11}$ with coupling coefficients $C_{V V h^{n}}=g_{V}^{2} v^{2-n} / 2 e^{2}\left(\right.$ where $g_{W}=g$ and $\left.g_{Z}=g \sec \theta_{W}\right)$.

Furthermore, the defined $W$ boson mass of Eq. (2.20) permits the Higgs vev $v$ to be experimentally determined. As $v$ is related to the $W$ mass in terms of the

\footnotetext{
${ }^{11}$ Here we define the metric $g_{\mu \nu}$ in the convention $(+,-,-,-)$. Throughout this work we will also define all couplings without overall factors of $e$ (see Appendix A).
} 
weak coupling constant $g$, it may also be related to the Fermi constant $G_{F} \cdot{ }^{12}$ This contact interaction between fermions describes an electroweak process (i.e. $\propto g^{2}$ ) that is mediated by the $W$ boson (i.e. $\propto 1 / m_{W}^{2}$ ). The experimental value of $G_{F}=$ $1.16639 \times 10^{-5} \mathrm{GeV}^{-2}[43]$ may therefore be used to set $v$,

$$
v=\left(\sqrt{2} G_{F}\right)^{-\frac{1}{2}}=\left(\frac{g^{2}}{4 m_{W}^{2}}\right)^{-\frac{1}{2}}=246 \mathrm{GeV} .
$$

This vev can then be used with the known $125 \mathrm{GeV}$ Higgs mass to set the parameters $\mu$ and $\lambda$ in the scalar potential by using Eq. (2.11) and $m_{h}^{2}=2 v^{2} \lambda$ from Eq. (2.13). The remaining two terms in Eq. (2.13) then define the Higgs self-couplings $-i C_{h h h^{n}}$ $(n=1,2)$, where $C_{h h h^{n}}=6 \lambda v^{2-n}=3 m_{h}^{2} / v^{n}$.

Fermions may also acquire mass due to the Higgs mechanism through Yukawa terms in the Lagrangian [48]. A Yukawa term $y_{f} \bar{\Psi} \phi \Psi$ describes the coupling of a Dirac fermion $(\Psi)$ to a scalar $(\phi)$. When the electroweak symmetry is broken $\left(\phi_{3} \rightarrow h+v\right)$, we obtain a fermion mass term $-m_{f} \bar{\Psi} \Psi$. For example, in the case of the leptons $L_{L}$, $\ell_{R}$ defined in Table 2.3, we have the following

$$
L_{\text {yuk }} \supset-y_{\ell}\left(\bar{\ell}_{R} \Phi^{\dagger} L_{L}+\bar{L}_{L} \Phi \ell_{R}\right)=-\frac{y_{\ell}}{\sqrt{2}}(h+v)\left(\bar{\ell}_{R} \ell_{L}+\bar{\ell}_{L} \ell_{R}\right)=-\frac{y_{\ell} v}{\sqrt{2}} \bar{\ell} \ell-\frac{y_{\ell}}{\sqrt{2}} h \bar{\ell} \ell,
$$

where the first term in the final equation is a fermion mass term for the unpolarized Dirac spinor $\ell$ (see Eq. (2.8)). Similar terms may be generated for the quarks. The fermion masses of Table 2.1 can therefore be reproduced with an appropriate choice of the Yukawa coupling constants $y_{f}=\sqrt{2} m_{f} / v$. Note that the Higgs must arise in a scalar electroweak doublet in order to form such Yukawa terms with the left-handed fermion doublets. The Yukawa terms also contribute to the breaking of the custodial symmetry, as this coupling of different electroweak representations does not preserve $S U(2)_{L} \times S U(2)_{R}$

\footnotetext{
${ }^{12}$ The Fermi constant $G_{F}$ is the coupling strength of the Fermi interaction, a four-fermion interaction proposed by Enrico Fermi in 1933 as part of his description of beta-decay [47].
} 


\subsection{Beyond the Standard Model}

The SM is an incredibly successful theory that has stood up to over five decades of rigorous experimental tests. However, there are still several questions and problems that it cannot successfully resolve. These issues have prompted particle theorists to move beyond the SM (BSM) and investigate models which incorporate new particles or mechanisms. Some of these BSM models simply introduce new particles to the theory under the same SM symmetry group, while others change the structure of the theory by using additional symmetries or changing the space-time structure of the theory (e.g. models with extra dimensions). However, as the predictions of the SM have been verified by decades of experimental measurements, most BSM theories must reduce to the SM in an appropriate low-energy limit.

\subsubsection{Standard Model difficulties and open questions}

A few of the more prominent difficulties and remaining questions with respect to the SM are briefly summarized below.

\section{The Hierarchy Problem}

A description of the gravitational force is conspicuously absent from the SM framework. Despite its ubiquity in our macroscopic lives, gravity is actually the weakest of the known fundamental forces, with an interaction strength 39 orders of magnitude smaller than that of the strong force. In comparison, the electromagnetic and weak forces are only smaller by 2 and 5 orders of magnitude respectively. As a result, the gravitational force is negligible at the electroweak scale (on the order of $100 \mathrm{GeV}$ ), 
and becomes relevant only at the Planck scale of $10^{19} \mathrm{GeV}$.

This large hierarchy in the strengths of the fundamental forces is unexplained by the SM, which is assumed to be an effective low-energy theory (as discussed in Sec. (2.2)). However, the hierarchy becomes problematic if the SM remains valid all the way up to the Planck scale. In this case, it is expected that the Higgs field would experience quantum fluctuations on the order of the Planck scale. If so, it is very odd that the Higgs vacuum expectation value and mass are so much smaller $(v=246$ $\mathrm{GeV}$ and $m_{h}=125 \mathrm{GeV}$ ), as this would imply that they are unnaturally fine-tuned to roughly 34 orders of magnitude.

As a result, it is expected that new physics must appear near to the $\mathrm{TeV}$ scale in order to suppress large quantum corrections to the Higgs mass. Many different BSM mechanisms have been designed to address this hierarchy problem. For

example, Supersymmetry (SUSY) [9, 10] and Little Higgs [52] models both implement additional symmetries that protect the size of the Higgs mass. Other models suppress the quantum corrections with the strongly-coupled dynamics of a composite Higgs boson [53-57] or by introducing extra dimensions of space-time [58-60].

\section{The mass spectrum and generations of the fermion sector}

There are also several unanswered questions in the fermion sector of the SM. Firstly, there is the large range of different fermion masses, which spans over five orders of magnitude (see Table 2.1). The SM can describe most of these masses by including Yukawa terms with an appropriate choice of coupling (see Eq. (2.22)), but it cannot explain why the fermion masses are so different.

Secondly, the SM cannot account for the non-zero — though tiny - masses 
of the neutrinos [61]. As there are no right-handed neutrinos in the SM (see Table 2.3), one cannot write a neutrino Yukawa term similar to the other fermions. If right-handed neutrinos exist, they may be much heavier than the left-handed neutrinos. The see-saw mechanism is a popular theoretical framework to describe this possibility [43]. In this mechanism neutrinos may receive mass from both Dirac mass terms - as in Eq. (2.22) — and Majorana mass terms of form $y_{m a j}\left(\bar{\nu}_{R} \nu_{R}^{c}+\bar{\nu}_{R}^{c} \nu_{R}\right){ }^{13}$ This leads to a mass matrix for the neutrinos with off-diagonal terms such that the right-handed neutrinos must become heavier as the left-handed neutrinos become lighter.

Finally, the SM does not provide a justification for the observed number of fermion generations. Although in can incorporate the three known generations of fermions, it does not explain why there should be exactly three. As the fermions increase in mass with generation, many physicists have questioned whether there might still be undiscovered generations of fermions. Alternatively, the number of generations may be a consequence of some larger BSM symmetry group (which would also generally require the existence of new particles).

\section{Dark matter}

Many cosmological measurements indicate that the known SM particles make up only a small fraction (less than 5\%) of the matter in the universe. While studying the Coma galaxy cluster in 1933, Franz Zwicky observed from the velocities of the galaxies that the mass of the cluster was much higher than one would expect from the emitted luminosity. He concluded that the cluster must contain a large amount of 'dark matter' that was not emitting visible radiation. Since then, many other

\footnotetext{
${ }^{13}$ Here the superscript $c$ denotes charge conjugation.
} 
cosmological measurements have produced experimental evidence for dark matter, including studies of other clusters, supernovae, galactic distributions, rotation curves, gravitational lensing, and the cosmic microwave background [62]. It is currently estimated that the universe contains five times as much dark matter as visible matter.

Many different explanations for dark matter have been proposed. The most popular theory is that dark matter is a BSM form of weakly-interacting massive particle (WIMP). Because the evidence for dark matter is indirect, very little is known about its properties; for example, the particles could be scalars, fermions, or axions. ${ }^{14}$ However, WIMP candidates must be massive and electrically neutral, as dark matter does not emit electromagnetic radiation. They must also be stable, as they would not be able to reproduce the observed abundance of dark matter in the universe if they could easily decay.

\subsubsection{BSM scalar extensions}

Many of these issues are intimately related to the Higgs mechanism. The difficulties involving the Higgs and the fermions both require explanations of particular mass values, while one of the few known properties of Dark Matter is that it is massive (suggesting that it may interact with a Higgs boson). As a result, many BSM models extend the scalar sector of the SM with new scalar particles or a larger electroweak symmetry group. The new scalar particles may be additional Higgs bosons if they have a vacuum expectation value, or they may be Dark Matter candidates if they are neutral and stable.

For example, a popular scalar extension is the Two Higgs Doublet Model (for

\footnotetext{
${ }^{14}$ Axions are hypothetical particles that were proposed as a solution to the strong CP problem.
} 
a recent review, see [8]). As the name suggests, this model simply includes two copies of the SM Higgs doublet, both of which acquire vacuum expectation values. As before, three of these degrees of freedom are rotated away to become the longitudinal polarizations of the electroweak gauge bosons. The remaining scalar particle spectrum then consists of two CP-even neutral scalars $h$ and $H,{ }^{15}$ a CP-odd neutral scalar $A^{0}$, and charged scalars $H^{ \pm}$. When extended with a supersymmetry, this model forms the base of many popular SUSY models, including the Minimal Supersymmetric Standard Model (MSSM).

In comparison, the Inert Doublet model also adds an electroweak scalar doublet to the usual SM scalar sector [12]. However, in this model the additional doublet is inert and does not acquire a vacuum expectation value. As a result, if the lightest state in the doublet is neutral, it will be a stable Dark Matter candidate.

Similarly, many other models have been developed using different sizes $(n)$ of electroweak scalar multiplets for a variety of purposes. In the interest of simplicity, historically most BSM models have added the minimal number of particles necessary to achieve a desired mechanism. As a result, many BSM models involve only electroweak $S U(2)$ singlets $[13,63,64]$, and doublets $[8,12]$. However, scalar triplet models $[17,30,64-69]$ have recently grown in popularity, and models with larger electroweak multiplets have also been developed. Scalar quadruplets $(n=4)$, quintuplets $(n=5)$ and sextets $(n=6)$ have all been used in models for neutrino mass generation [18-29], while scalar septets $(n=7)$ have been considered in a Higgs model [31-33]. Quintuplets [14,15] and septets [16] have also made appearances in dark matter models.

This thesis will focus on models that extend the SM scalar sector only by one

\footnotetext{
${ }^{15}$ The $h$ state is usually assumed to be the SM-like $125 \mathrm{GeV}$ scalar state.
} 
or more electroweak scalar multiplets of size $n \geq 3$. In particular, it will consider two classes of models with large inert multiplets of size $n \geq 5[2,3]$, as well as the Georgi-Machacek model (GM), which includes scalar triplets [30,70]. We will focus on the ability of these models to provide explanations and benchmarks for dark matter, exotic scalars, and altered Higgs couplings. These mechanisms and the signatures of simplified scalar extensions can be incorporated into more complex and complete theories. As we are not focusing on mechanisms for a unified theory or explanations for the Hierarchy problem, we will not explicitly consider models with extended symmetry groups $[9,10,52]$, other types of additional particles (i.e. fermions, gauge bosons), composite particles [53-57], or extra dimensions [58-60].

\subsubsection{Current experimental status of scalar particles}

In 2012, the ATLAS and CMS experimental collaborations announced the discovery of a new $125 \mathrm{GeV}$ particle at CERN's Large Hadron Collider [71,72]. Further analysis of this particle by both collaborations lead to the conclusion that it is a CP-even scalar particle consistent with the SM Higgs boson $[73,74]$.

In the subsequent years, many interactions and decays of this Higgs particle have been tested, and to date all of the experimental data is consistent with the $\mathrm{SM}[44,73,74,76] \cdot{ }^{16}$ A summary of the experimental status of these measurements is illustrated in Fig. (2.1). These plots show the $1 \sigma$ range of the measured signal for each process as a ratio to the SM prediction. One will note that all of the measurements are well within $2 \sigma$ of the SM prediction of 1 .

In other words, no additional scalar particles have been discovered to date and

\footnotetext{
${ }^{16}$ In this context, 'consistent' means the SM predictions are within two standard deviations of the experimental measurements.
} 

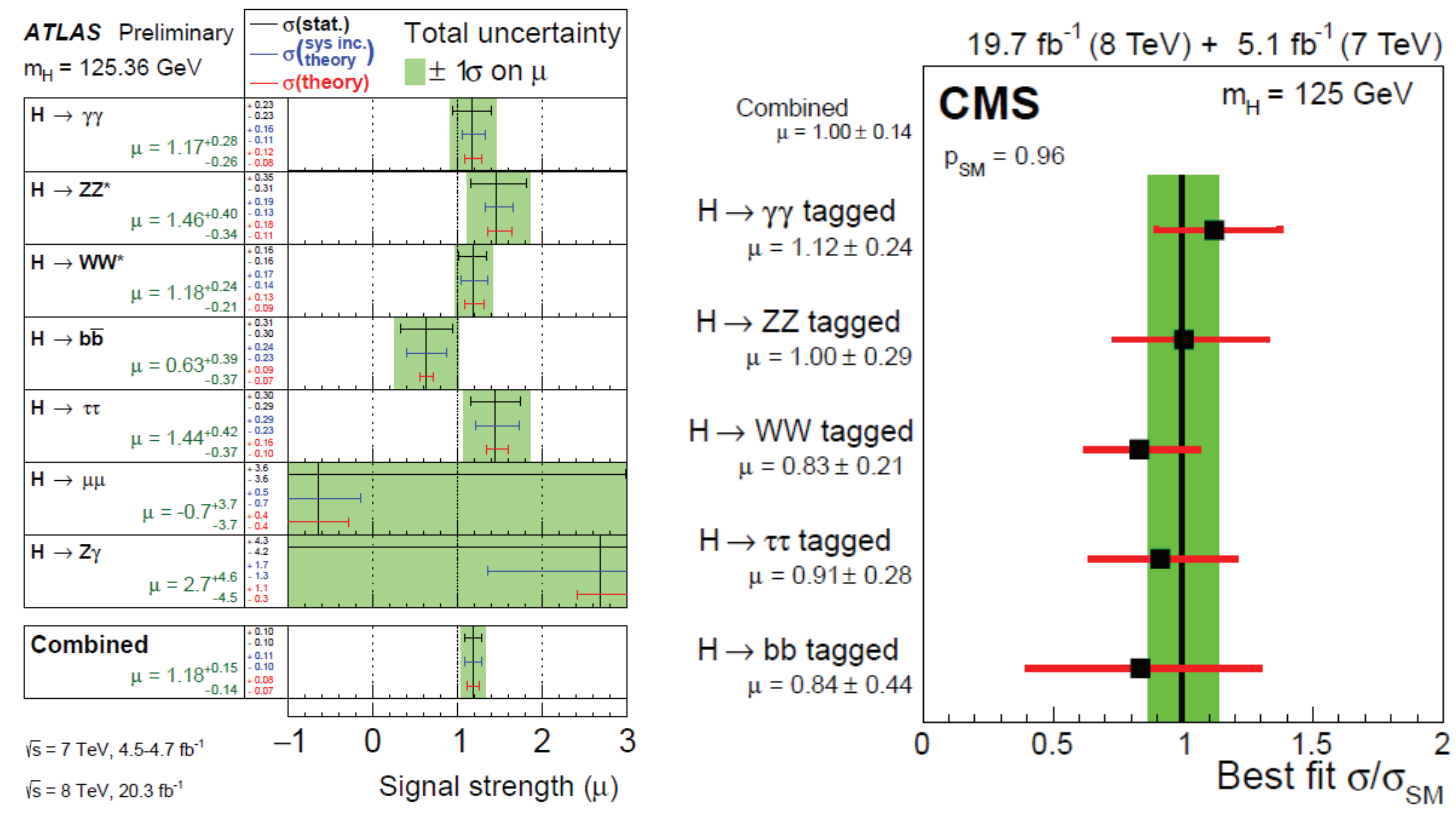

Figure 2.1: The current experimental status of Higgs decay modes. The cross section for each final state is normalized to its SM prediction. Left: ATLAS results [75]. Right: CMS results. [76]. These plots are reproduced unaltered from the cited references under the CC-BY-3.0 license [C 2015 CERN].

no indications of new physics have been observed in the Higgs interactions. These SM-like results can place considerable constraints on the masses and couplings of BSM scalar particles. However, as most BSM models either reduce to or approximate the SM predictions in an appropriate limit or region of parameter space, they generally cannot be entirely excluded while the SM remains viable. As a result, investigations of the model phenomenology as one approaches these SM-like limits are necessary in order to identify techniques for differentiating BSM models from the SM. Given the varied motivations for moving beyond the SM, it is also very important that both theorists and experimentalists carefully consider additional mechanisms that could hide the effects of BSM physics within experimental results from colliders. 


\section{Chapter 3}

\section{Constraints on models with scalar multiplet extensions}

In analysing beyond-the-Standard-Model (BSM) models with extensions of the scalar sector, it is important to consider several categories of questions:

- Can this model be constrained by theoretical considerations (i.e. does the model break down in certain regions of parameter space)?

- What constraints can be placed upon this model from experimental data?

- Does this model reduce to the Standard Model (SM) in some limit? Is there a conclusive method of differentiating it from the SM?

The relevant constraints and considerations required for the purposes of this thesis are discussed in general terms in this chapter. Detailed discussions of the application of these constraints in the context of particular models can be found in Chapters 4-7. 


\subsection{Theoretical constraints}

\subsubsection{Tree-level perturbative unitarity}

In working with BSM scalar theories, we will require that the models obey perturbative partial-wave unitarity at tree level. This translates into an assumption that all particles interact weakly (i.e. all coupling strengths are $\leq 1$ ), as a violation of unitarity indicates that the model becomes strongly coupled at some scale. If unitarity is violated the perturbation theory required to perform calculations breaks down, as a fixed order of Feynman diagrams cannot be used to calculate the model predictions if higher-order Feynman diagrams become large. The application of a strongly-coupled scalar theory would be in a composite Higgs boson model; as this is not the purpose of the models considered below, we will assume that tree-level unitarity is preserved.

In order to evaluate the unitarity limits on a model with large scalar multiplet, we compute the $2 \rightarrow 2$ tree-level scattering amplitudes for scalar pair annihilation into scalars or electroweak gauge bosons. The partial wave amplitudes are related to the scattering matrix elements according to

$$
\mathcal{M}=16 \pi \sum_{J}(2 J+1) a_{J} P_{J}(\cos \theta),
$$

where $J$ is the orbital angular momentum of the final state and $P_{J}(\cos \theta)$ is the corresponding Legendre polynomial. Tree-level partial wave unitarity dictates that

$$
\left|\operatorname{Re} a_{0}\right| \leq 1 / 2
$$

We will use only the zeroth partial wave amplitude, $a_{0}$, to set our unitarity limits. As a result, we need only calculate the contributions to the amplitude $\mathcal{M}$ that are constant in the angle $\theta, \mathcal{M}_{0}$. We define

$$
\mathcal{M}_{0}=16 \pi a_{0}
$$




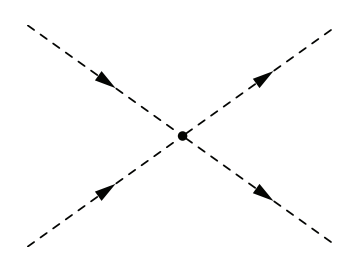

(a)

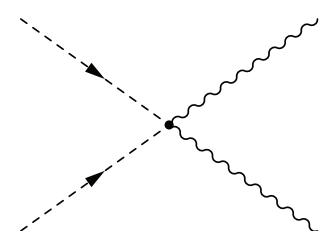

(b)

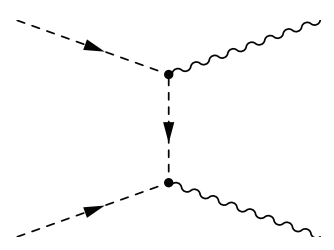

(c)

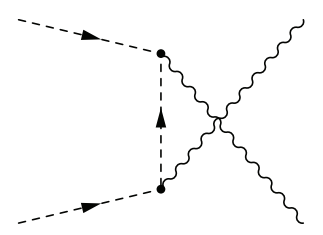

(d)

Figure 3.1: Feynman diagrams contributing to $s_{1} s_{2} \rightarrow s_{3} s_{4}$ and $s s^{*} \rightarrow V_{1} V_{2}$.

We compute these amplitudes in the high-energy limit, as the unitarity bound must be preserved regardless of energy scale. As a result, we neglect gauge boson masses, and replace the longitudinal degrees of freedom in the gauge bosons with scalar Goldstone bosons using the Goldstone Equivalence Theorem [48]. The highenergy limit is useful in that it allows us to neglect many diagrams which are suppressed by the collision energy. It further allows us to neglect the effects of electroweak symmetry breaking; if desired, we may choose to work in the unmixed $S U(2)_{L} \times U(1)_{Y}$ basis of $W^{a}$ and $B(a=1,2,3)$ gauge bosons so that the $S U(2)_{L}$ interactions are distinct from the $U(1)_{Y}$ hypercharge interactions.

In applying these unitarity limits we will consider two categories of amplitudes: the scattering of two scalars to two scalars and the scattering of two scalars to two vector bosons. Amplitudes with only vector bosons in the initial and final states may be neglected in the cases we consider; those with scalar propagators will be suppressed by the collision energy, while those with vector boson propagators will be suppressed by the weak coupling $g^{2}$ without being enhanced by the number of scalars available.

\section{Unitarity bounds from scalar-to-scalar scattering amplitudes}

In the first case of scalar-to-scalar scattering $\left(s_{1} s_{2} \rightarrow s_{3} s_{4}\right)$ the dominant diagrams that contribute to $a_{0}$ are quartic scalar interactions, as shown in Fig. 3.1a. Diagrams 
with scalar propagators will be suppressed by the collision energy and neglected in the high-energy limit, while diagrams with vector boson propagators are suppressed by $g^{2}$ and may be neglected in comparison with the quartic couplings in the models that we consider. Additionally, diagrams with s-channel vector boson propagators will be proportional to $\cos \theta$, and therefore will contribute only to $a_{1}$ in Eq. (3.1), not $a_{0}$. Therefore the scalar-to-scalar amplitudes will be given by

$$
\mathcal{M}_{0}\left(s_{1} s_{2} \rightarrow s_{3} s_{4}\right)=-C_{s_{1} s_{2} s_{3} s_{4}}
$$

where $C_{s_{1} s_{2} s_{3} s_{4}}$ is the quartic coupling coefficient of the four scalars.

\section{Unitarity bounds from scalar-to-vector scattering amplitudes}

In the case of scalar-to-vector scattering, we consider only neutral processes in the work that follows. As a result, we consider only scalar anti-scalar pairs of the same species $\left(s s^{*} \rightarrow V_{1} V_{2}\right)$, as the high-energy limit allows us to neglect the effects of electroweak symmetry breaking, including any mixing between the scalar states. Three different forms of Feynman diagrams are relevant to these calculations. They consist of a quartic coupling (Fig. 3.1b), a t-channel interaction with a scalar propagator (Fig. 3.1c), and the associated u-channel interaction with a scalar propagator (Fig. 3.1d). Diagrams with vector boson or s-channel scalar propagators will either be suppressed by the collision energy or proportional to $\cos \theta$ (i.e. contribute to $a_{1}$ ).

Working in the high-energy limit, we consider only the transverse polarizations of the gauge bosons as the longitudinal components will be replaced by scalar Goldstone bosons. There are four distinct polarization combinations: both gauge bosons polarized in the scattering plane, both out of the scattering plane, $V_{1}$ in and $V_{2}$ out, and $V_{2}$ in and $V_{1}$ out. When the two gauge bosons have different polarizations, the 
matrix element is zero. However, the two cases where the polarizations are the same give identical matrix elements in the high-energy limit.

Specifically, let us define the scattering process to be in the $(x, z)$ plane, with the following four-momenta:

$$
\begin{array}{ll}
p_{1}^{\mu}=\left(E_{p_{1}},\left|\vec{p}_{1}\right| \sin \theta, 0,\left|\vec{p}_{1}\right| \cos \theta\right), & k_{1}^{\mu}=\left(E_{k_{1}}, 0,0,\left|\vec{k}_{1}\right|\right), \\
p_{2}^{\mu}=\left(E_{p_{2}},-\left|\vec{p}_{2}\right| \sin \theta, 0,-\left|\vec{p}_{2}\right| \cos \theta\right), & k_{2}^{\mu}=\left(E_{k_{2}}, 0,0,-\left|\vec{k}_{2}\right|\right),
\end{array}
$$

where $p_{1}$ and $p_{2}$ are the incoming four-momenta of $s$ and $s^{*}, k_{1}$ and $k_{2}$ are the outgoing four-momenta of $V_{1}$ and $V_{2}$, respectively, and $\theta$ is the scattering angle. When both vector bosons are polarized out of the scattering plane, with polarization vectors

$$
\epsilon_{\text {out }}^{\mu}\left(k_{1}\right)=(0,0,1,0) \quad \text { and } \quad \epsilon_{\text {out }}^{\mu}\left(k_{2}\right)=(0,0,-1,0) \text {, }
$$

only the diagram of Fig. 3.1b is non-zero. Therefore the total amplitude contribution to $a_{0}$ in the case of out-of-plane-polarized vector bosons is

$$
\mathcal{M}_{0}^{\text {ssVV,out }}=\mathcal{M}_{\text {quartic }}=e^{2} C_{s s^{*} V_{1} V_{2}}
$$

where $e^{2} C_{s s^{*} V_{1} V_{2}}$ is the quartic coupling coefficient between the two scalars and two vector bosons (as defined in Appendix A), and $\mathcal{M}_{\mathrm{t}-\text { channel }}=\mathcal{M}_{\mathrm{u}-\text { channel }}=0$. Similarly, when the bosons are polarized in the scattering plane, with polarization vectors

$$
\epsilon_{\mathrm{in}}^{\mu}\left(k_{1}\right)=(0,1,0,0) \quad \text { and } \quad \epsilon_{\mathrm{in}}^{\mu}\left(k_{2}\right)=(0,1,0,0),
$$

all three diagrams are non-zero. In this case the amplitudes are given by

$$
\begin{aligned}
\mathcal{M}_{\text {quartic }} & =-e^{2} C_{s s^{*} V_{1} V_{2}}, \\
\mathcal{M}_{\mathrm{t}-\text { channel }} & =e^{2} C_{s s_{1}^{*} V_{1}} C_{s_{1} s^{*} V_{2}}(1+\cos \theta), \\
\mathcal{M}_{\mathrm{u}-\text { channel }} & =e^{2} C_{s s_{1}^{*} V_{2}} C_{s_{1} s^{*} V_{1}}(1-\cos \theta),
\end{aligned}
$$


where $s_{1}$ is the propagating scalar in the $t$ and $u$-channel diagrams. As the parts of $\mathcal{M}$ that are proportional to $\cos \theta$ in the t-channel and $u$-channel cases contribute to the partial wave $a_{1}$ (see Eq. (3.1)), the total amplitude contribution to $a_{0}$ is given by

$$
\mathcal{M}_{0}^{\mathrm{ssVV}, \text { in }}=e^{2}\left(-C_{s s^{*} V_{1} V_{2}}+C_{s s_{1}^{*} V_{1}} C_{s_{1} s^{*} V_{2}}+C_{s s_{1}^{*} V_{2}} C_{s_{1} s^{*} V_{1}}\right) .
$$

However, due to symmetries in the couplings for neutral interactions of scalars with gauge bosons, in general $2 C_{s s^{*} V_{1} V_{2}}=C_{s s_{1}^{*} V_{1}} C_{s_{1} s^{*} V_{2}}+C_{s s_{1}^{*} V_{2}} C_{s_{1} s^{*} V_{1}}$. Therefore the total in-plane-polarized contribution is $\mathcal{M}_{0}^{\text {in }}=e^{2} C_{s s^{*} V_{1} V_{2}}$, and we find in general that

$$
\mathcal{M}_{0}\left(s s^{*} \rightarrow V_{1} V_{2}\right)=e^{2} C_{s s^{*} V_{1} V_{2}}
$$

for gauge bosons with identical polarizations. These amplitudes will be altered by a symmetry factor of $1 / \sqrt{2}$ when the incoming or outgoing particles are identical.

\section{Unitarity bounds from coupled amplitudes}

When nonzero amplitudes exist that couple the same initial (final) state to multiple final (initial) states, the individual amplitudes are not linearly independent. In this case applying Eq. (3.2) to an individual amplitude will not necessarily give an appropriate bound, as it neglects contributions that could cause an increase or decrease to the amplitude in another basis of states. The strongest constraint will come from applying the bound to the largest linearly independent amplitude. We therefore construct a matrix of the amplitudes $\mathcal{M}_{0}$ - in the basis of the initial and final states and apply Eq. (3.2) to the largest eigenvalue of that matrix.

As electric charge is always conserved, distinct matrices can be constructed by collecting all amplitudes of a common total electric charge in the initial or final state. In general the highest-dimensional unitarity matrix of amplitudes will be that 
of interactions that are neutral overall; this neutral matrix will therefore be the most important for applying constraints based on unitarity. In the models we consider, the matrices of charged amplitudes duplicate the relevant constraints from the matrix of neutral amplitudes.

\section{Unitarity bounds involving scalar multiplets of various sizes}

In the following work we consider constraints on models where the size of the scalar multiplet may vary. As a result, it will be helpful to place constraints from unitarity on a scalar multiplet of generic size $n$, where the size is defined in terms of the isospin $T$ by $n=2 T+1$ (and $n$ is the number of isospin components).

As described in the previous section, when applying these bounds we would normally calculate the individual neutral amplitudes and then assemble a matrix in the basis of the initial and final states. Without a definite number of scalars, this neutral amplitude matrix will have an indeterminate size, making it difficult to assemble explicitly.

However, since Eqs. (3.4) and (3.11) depend only on coupling coefficients, amplitudes involving different scalar species from the multiplet will differ only in their isospin components, $T^{ \pm}$and $T^{3}$. These quantum numbers can be expressed in

terms of the total isospin $T=\frac{n-1}{2}$ and the placement $k$ of the scalar in the multiplet, i.e. $s_{k}$ is the $k^{\text {th }}$ component of the generic multiplet

$$
\mathcal{S}^{T}=\left(s_{n}, \ldots, s_{1}\right)
$$

Furthermore, because Eqs. (3.4) and (3.11) depend only on quartic couplings, we need only consider the operator combinations $T^{3}$ and $\left[T^{+} T^{-}+T^{-} T^{+}\right]$. Operating on a scalar multiplet $\mathcal{S}$ these operators give the following results, 


$$
\begin{gathered}
T^{3} \mathcal{S}=T_{k}^{3} s_{k}=(k-1-T) s_{k}=\left(k-\frac{n+1}{2}\right) s_{k} \\
{\left[T^{+} T^{-}+T^{-} T^{+}\right] \mathcal{S}=2\left[T(T+1)-\left(T^{3}\right)^{2}\right] \mathcal{S}=2\left[T(T+1)-\left(k-\frac{n+1}{2}\right)^{2}\right] s_{k}}
\end{gathered}
$$

In the high-energy limit, scattering processes conserve isospin as we neglect the effects of electroweak symmetry breaking. Using Eqs. (3.13) and (3.14), the neutral amplitudes can be grouped by total isospin using the following normalized linear combinations of scalar fields

$$
\begin{aligned}
& {\left[s^{*} s\right]_{0}=\frac{1}{\sqrt{n}} \sum_{k} s_{k}^{*} s_{k},} \\
& {\left[s^{*} s\right]_{1}=\frac{\sum_{k} s_{k}^{*} T^{3} s_{k}}{\left[\sum_{k}\left(T^{3}\right)^{2}\right]^{1 / 2}}=\frac{\sum_{k} s_{k}^{*} T^{3} s_{k}}{\left[n\left(n^{2}-1\right) / 12\right]^{1 / 2}},} \\
& {\left[s^{*} s\right]_{2}=\frac{\sum_{k} s_{k}^{*}\left[T(T+1)-3\left(T^{3}\right)^{2}\right] s_{k}}{\left[\sum_{k}\left\{T(T+1)-3\left(T^{3}\right)^{2}\right\}\right]^{1 / 2}}=\frac{\sum_{k} s_{k}^{*}\left[T(T+1)-3\left(T^{3}\right)^{2}\right] s_{k}}{\left[n\left(n^{2}-1\right)\left(n^{2}-4\right) / 20\right]^{1 / 2}},}
\end{aligned}
$$

where the subscripts $0,1,2$ denote the total isospin, and the summation index $k=$ $1, . ., n .^{1}$ These combinations are by definition orthogonal to each other.

The vector boson fields can similarly be grouped into $B B / \sqrt{2}$ (isospin 0), $B W^{3}$ (isospin 1), and $W$ combinations of

$$
\begin{aligned}
& {[W W]_{0}=\frac{1}{\sqrt{3}}\left[\sqrt{2}\left(W^{+} W^{-}\right)+\left(W^{3} W^{3} / \sqrt{2}\right)\right],} \\
& {[W W]_{2}=\frac{1}{\sqrt{3}}\left[\left(W^{+} W^{-}\right)-\sqrt{2}\left(W^{3} W^{3} / \sqrt{2}\right)\right] .}
\end{aligned}
$$

Using Eqs. (3.13)-(3.19), we can calculate the amplitudes corresponding to initial and final states that are linear combinations with the same isospin, e.g. $M_{0}\left(\left[s^{*} s\right]_{0} \rightarrow[W W]_{0}\right)$ or $M_{0}\left(\left[s^{*} s\right]_{1} \rightarrow W^{3} B\right)$. We may then construct three

\footnotetext{
${ }^{1}$ Eq. (3.17) uses $\sum_{j=1}^{N} j^{2}=N(N+1)(2 N+1) / 6$ and $\sum_{j=1}^{N} j^{4}=N(N+1)(2 N+1)\left(3 N^{2}+3 N-1\right) / 30$.
} 
distinct matrices based on isospin, in the bases of the linear combinations of fields. This procedure therefore reduces our original neutral amplitude matrix of varying size $n$ to three manageable matrices of definite size, greatly simplifying the process of determining the eigenvalues.

In Chp. 4, the size and hypercharge of an $S U(2)$ scalar multiplet will be bounded through an application of tree-level perturbative unitarity to the neutral interactions of scalars and electroweak gauge bosons (see Sec. 4.2). Unitarity will further be used to constrain the parameter space of $U(1)$ and $Z_{2}$-symmetric models with large scalar multiplets in Chps. 5-6 (see Secs. (5.2.1 and 6.2.1 respectively), as well as the Georgi-Machacek (GM) model in Chp. 7 (see Sec. 7.2.1).

\subsubsection{Bounded-from-below constraints}

In order to implement the Higgs mechanism, the scalar potential of a model must have a non-zero minimum that breaks the electroweak symmetry. As such, the scalar potential must be bounded from below, a requirement which constrains the parameters of the scalar potential $\lambda_{i}$.

In the following, we will generally write down scalar potential terms that are up to quartic order in the scalar fields; higher-order terms will be omitted, as such terms are non-renormalizable. In this case, the constraints that must be satisfied at tree level for the scalar potential to be bounded from below can be determined by considering only the terms in the scalar potential that are quartic in the fields, as these terms dominate at large field values. By applying an appropriate parametrization of the fields, the potential may often be written in a simplified way, ideally in a quadratic or quartic form where the requirements for boundedness are well known. This allows 
us to more easily assess the limits imposed on the parameters by the requirement of boundedness. Of course, the exact form of this parametrization will depend on the potential of the model in question.

In general the scalar potential may be parametrized as follows [69]:

- Define a dimensionful variable $r^{2}$ as the sum of the fields in quadrature; for example, for two multiplets $S_{1,2}$ we would define $r^{2}=\operatorname{Tr}\left(S_{1}^{\dagger} S_{1}\right)+\operatorname{Tr}\left(S_{2}^{\dagger} S_{2}\right)$.

- Define the absolute square of each field using the variable $r$ and an angular variable, e.g. $\operatorname{Tr}\left(S_{1}^{\dagger} S_{1}\right)=r^{2} \cos \alpha$.

- Define any remaining terms in the potential which cannot be parametrized by $r$ and $\alpha$ alone in terms of additional dimensionless variables. This can be achieved by dividing the term by a factor $\sqrt{\operatorname{Tr}\left(S_{i}^{\dagger} S_{i}\right)}$ for each field $S_{i}$ that appears within the term. For example, the term $\operatorname{Tr}\left(S_{1}^{\dagger} S_{1} S_{1}^{\dagger} S_{1}\right)$ can be parametrized as $\omega r^{4} \cos ^{2} \alpha$ using the dimensionless variable $\omega=\operatorname{Tr}\left(S_{1}^{\dagger} S_{1} S_{1}^{\dagger} S_{1}\right) /\left(\operatorname{Tr}\left(S_{1}^{\dagger} S_{1}\right)\right)^{2}$ and the definitions of $r$ and $\alpha$ above.

- As the only dimensionful variable is $r$ - and all potential terms must have the same dimension of $\left[r^{4}\right]$ — the quartic scalar potential should then be expressible in a form proportional to $r^{4}$ and some combination of the potential parameters $\lambda_{i}$, the angular variables $\alpha_{i}$, and the dimensionless variables $\omega_{i}$, e.g. $V_{\text {quartic }}=$ $r^{4} A\left(\lambda, \alpha_{i}, \omega_{i}\right)$. This potential will be bounded from below when $A\left(\lambda_{i}, \alpha_{i}, \omega_{i}\right)>$ 0. Ideally $A$ will have a simple form (e.g. quadratic, biquadratic) where the conditions for $A$ to be positive are known and may easily be applied to constrain combinations of $\lambda_{i}, \alpha_{i}$, and $\omega_{i}$.

- By scanning over all possible field values of the multiplet scalars, one may deter- 
mine the parameter ranges for the dimensionless variables $\alpha_{i}$ and $\omega_{i}$. These will in turn allow us to extract the maximum allowed ranges of the scalar potential parameters $\lambda_{i}$, as desired.

Constraints from the boundedness of the potential will be applied to the GM model in Chp. 7 (see Sec. 7.2.2).

\subsubsection{Alternative vacua}

Ideally, the electroweak-symmetry-breaking (EWSB) minimum of the scalar potential should also be the global minimum. If the minimum is only local, the EWSB vacuum may be meta-stable as the deeper global minimum will be a preferred state for the physical system. If the true global minimum does not preserve the appropriate EWSB mechanism — for example, if it breaks important additional symmetries such as the $U(1)_{\mathrm{EM}}$ electromagnetic symmetry - then the resulting model may only be functional for a finite lifetime (if at all).

In order to check that the desired EWSB vacuum is the global minimum of the scalar potential, one must minimize the scalar potential $V$ and determine the corresponding value of $V$ for the desired vacuum. One then repeats the process for every possible combination of scalar field values in the model. If the value of $V$ at the desired vacuum is lower than that at any other field configuration, then we are assured that the desired vacuum is the true global minimum of the potential.

In some cases this requirement may be used to obtain analytic bounds on the parameters of the scalar potential. However, in general it will be most easily implemented in the form of a numeric scan over a model's parameter space. This can be done straightforwardly by parametrizing the full scalar potential in a manner 
similar to the procedure outlined in the previous section:

- Parametrize all terms in the scalar potential using a dimensionful variable $r$, angular variable $\alpha_{i}$, and dimensionless variables $\omega_{i}$ as described in Sec. (3.1.2). Determine the allowed ranges of all variables by scanning over all field values.

- Determine the values of the variables that correspond to the desired electroweaksymmetry-breaking vacuum.

- Choose a set of values for the scalar potential parameters $\lambda_{i}$. Minimize $V$ using the values of $r, \alpha_{i}$, and $\omega_{i}$ that correspond to the desired vacuum.

- With the same $\lambda_{i}$, minimize $V$ for all other allowed values of $r, \alpha_{i}$, and $\omega_{i}$.

- If the potential minimum $V_{\min }^{\mathrm{EW}}$ corresponding to the electroweak vacuum is lower than all other alternatives $V_{\min }^{\text {alt }}$, then the chosen set of $\lambda_{i}$ values is safe from the possibility of alternative vacua.

These constraints will be applied to the GM model in Chp. 7 (see Sec. 7.2.3).

\subsection{Experimental constraints}

BSM theories with additional scalar particles can additionally be constrained by a variety of experimental data. Direct collider constraints arise from the fact that collider searches have yet to turn up direct evidence of such particles. Models which include a dark matter candidate may further be constrained by data from cosmological and direct-detection dark matter experiments. However, the majority of the experimental constraints considered in this thesis fall into the category of indirect constraints. These are constraints from measured observables that would be indirectly affected by 
the presence of new scalar particles (but do not require the direct production of such particles). These indirect constraints typically come from virtual corrections to SM processes due to new scalars in a quantum loop.

\subsubsection{Direct collider searches}

As summarized in the preceding chapter, to date no BSM scalars have been directly observed in experiments. In some cases, this absence of direct observation can be translated into constraints on scalar properties. In general, these limits are very model-dependent, as they depend upon the ability of a particular experiment to isolate and identify model-specific signatures. Most direct search constraints can be evaded by particular types of phenomenology or clever model-building. However, some limits are general enough in their scope to be applicable to most BSM theories. We will use these bounds to guide the lower limits of the BSM scalar masses we consider in all of the following work.

\section{$Z$ boson decay}

With respect to BSM scalars, the most general direct collider constraint arises from $Z$ decay, which has been very precisely measured at the Large Electron-Positron collider (LEP-I) and the Stanford Linear Collider (SLC) [43,77]. The total $Z$ decay width was measured directly from the resonance line shape and was found to agree with the SM prediction to an extremely high precision. Furthermore, the $Z$ branching ratios were also found to be in close agreement with SM predictions; in particular, the measured rate of $Z$ decays to invisible ${ }^{2}$ particles closely matches the expected rate of $Z \rightarrow \nu \bar{\nu}$.

\footnotetext{
${ }^{2}$ Decays to particles that remain undetected by the experiment - usually because they are electrically neutral - can introduce a large amount of uncertainty into decay measurements.
} 
The precision of the LEP measurements requires that new particles which couple to the $Z$ must be heavier than about $m_{Z} / 2$ so that they cannot contribute to $Z$ decay. This places a strict lower bound of $45 \mathrm{GeV}$ on the masses of most BSM scalars. ${ }^{3}$

\section{Higgs decay}

Scalars with masses below $m_{h} / 2 \simeq 63 \mathrm{GeV}$ can further be constrained by measurements of the Higgs decay width at the Large Hadron Collider (LHC). The contribution to this width from $h \rightarrow s s^{*}$ is given by

$$
\Gamma\left(h \rightarrow s s^{*}\right)=\frac{v^{2} C_{h s s^{*}}}{16 \pi m_{h}} \sqrt{1-\frac{4 m_{s}^{2}}{m_{h}^{2}}} \text { for } m_{s} \leq m_{h} / 2,
$$

where $C_{h s s^{*}}$ is the coupling coefficient for the coupling of a BSM scalar particle $s$ and anti-particle $s^{*}$ to the $125 \mathrm{GeV}$ Higgs $h$. As the Higgs has not been observed to decay to new stable charged particles, charged BSM scalars must either be heavier than this mass limit or must decay very quickly. In the case that $s$ is neutral, this contribution is constrained by the current upper limit of $B R(h \rightarrow$ invisible $) \leq 0.295$ [78] on the branching ratio of the Higgs to invisible particles, where the branching ratio for Higgs decay to a final state $X$ is defined by ${ }^{4}$

$$
B R(h \rightarrow X)=\frac{\Gamma(h \rightarrow X)}{\Gamma(h \rightarrow \text { all })}
$$

This limit arises from direct searches for $p p \rightarrow Z h$ at the LHC.

Certain limits may also be obtained from the fact that the current measurements of Higgs decay rates are consistent with the Standard Model. The rate at which

\footnotetext{
${ }^{3}$ A lone CP-even or CP-odd neutral scalar may be lighter than $m_{Z}$ as the kinematic structure of the $Z$ coupling to scalars prevents it from coupling to two identical particles.

${ }^{4}$ Here $\Gamma(h \rightarrow X)$ is the Higgs partial decay width to a final state $X$ and $\Gamma(h \rightarrow$ all $)$ is the total Higgs decay width. The total decay width of a particle is the sum of all partial decay widths, and is inversely proportional to its lifetime, $\Gamma_{t o t}=1 / \tau$.
} 
one would observe the Higgs decay to a final state $X(h \rightarrow X)$ in a given experiment is proportional to the cross section for Higgs production $\sigma_{h}$ (i.e. how many Higgs bosons will be produced for a given luminosity) times the $h \rightarrow X$ branching ratio (i.e. the probability that a Higgs will decay to $X$ ). As a ratio to the SM, this rate or signal strength - is defined as

$$
\mu_{X}=\frac{\sigma_{h} B R(h \rightarrow X)}{\sigma_{h}^{S M} B R(h \rightarrow X)_{S M}},
$$

In many BSM models the effect of the BSM scalar states on certain Higgs interactions can be negligible if the new states contribute only at loop-level in comparison with tree-level SM contributions. If both the Higgs production modes and the Higgs decay width $h \rightarrow X$ are SM-like - i.e. $\sigma_{h} \approx \sigma_{h}^{S M}$ and $\Gamma(h \rightarrow X) \approx \Gamma(h \rightarrow X)_{\mathrm{SM}}$ - then the branching ratio of the Higgs to new particles can be related to the signal strength of any Higgs decay channel $\mu_{X}$,

$$
\begin{aligned}
\mu_{X} & \simeq \frac{B R(h \rightarrow X)}{B R(h \rightarrow X)_{S M}}=\frac{\Gamma(h \rightarrow X)}{\Gamma_{\text {tot }}^{S M}+\Gamma_{\text {new }}} \frac{\Gamma_{\text {tot }}^{S M}}{\Gamma(h \rightarrow X)_{\mathrm{SM}}} \\
& \simeq 1-\frac{\Gamma_{\text {new }}}{\Gamma_{\text {tot }}^{S M}+\Gamma_{\text {new }}}=1-\operatorname{BR}(h \rightarrow \text { new }),
\end{aligned}
$$

where we have assumed that $\Gamma_{\text {new }}$ is small in comparison with $\Gamma_{\text {tot }}^{S M} \simeq 4.1 \mathrm{MeV}$ for $m_{h}=125 \mathrm{GeV}$ [79]. Using Eq. (3.23), a rough lower bound on $\mathrm{BR}(h \rightarrow$ new) can be obtained from Higgs decays to $W W$ and $Z Z$, where $\mu_{X} \gtrsim 0.41[75,76]$.

\section{Electron positron scattering}

Finally, CERN's LEP-II experiment performed a variety of direct searches for BSM particles produced by electron-positron scattering [80-83]. The experimental limits on these scattering processes can be translated into lower bounds of up to about $100 \mathrm{GeV}$ on some BSM scalar masses, depending on the preferred decay modes. Furthermore, 
the original searches were often designed for particular models (e.g. SUSY, 2HDM). Consequently, the resulting limits are model-dependent and are therefore best viewed as very rough guidelines when applied to other BSM scalars in the absence of a model-specific collider study.

\subsubsection{Constraints from the $\rho$ parameter at tree level}

One of the most stringent constraints on scalar multiplets under the $S U(2)_{L}$ symmetry is that of the $\rho$-parameter. This parameter is defined as the ratio of the charged and neutral electroweak currents. It effectively represents the relationship between the masses of the $W^{ \pm}$and $Z$ bosons,

$$
\rho=\frac{m_{W}^{2}}{m_{Z}^{2} \cos ^{2} \theta_{W}} .
$$

It is therefore intimately related to custodial symmetry, the approximate $S U(2)_{c}$ symmetry which results in the $m_{W}=m_{Z} c_{W}$ relationship of the SM. Models which preserve this symmetry in the scalar potential predict that $\rho=1$ at tree-level.

The contribution to the observable $\rho$ from electroweak scalar multiplets is

$$
\rho=\frac{\sum_{k} 2 v_{k}^{2}\left[T_{k}\left(T_{k}+1\right)-Y_{k}^{2} / 4\right]}{\sum_{k} Y_{k}^{2} v_{k}^{2}} .
$$

Here $v_{k}, T_{k}$, and $Y_{k}$ are the vacuum expectation value (vev), weak isospin, and hypercharge of the $k$ th multiplet in the model. Note that the contributions to $\rho$ depend on the vev $v_{k}$ of the multiplet. Scalar multiplets without vevs - such as may be included in a model of dark matter, for example - will not contribute to the $\rho$-parameter. However, in the SM the scalar doublet carries a non-zero vev; note that with the Higgs values of $v_{k}=v_{S M}, T_{k}=1 / 2$, and $Y=1$, Eq. (3.25) becomes $\rho=1$. This is true for any model that includes only scalar doublets with $Y=1$. Eq. (3.25) is 
similarly equal to one for a septet with $Y=4$, while a singlet with $Y=0$ will not constribute to either the numerator or denominator.

Experiments have confirmed that the $\rho$ parameter is in extremely close agreement with the SM prediction of 1, with an experimental value of [43]

$$
\rho=1.00040 \pm 0.00024
$$

The high precision on this measurement places stringent constraints on BSM electroweak scalars, as most scalar multiplets with isospin $T \geq 1(n \geq 3)$ will contribute to the $\rho$ parameter if they have a non-zero vev.

The $U(1)$ and $Z_{2}$-symmetric models with large scalar multiplets that are considered in Chps. 5-6 are not subject to constraints from the $\rho$-parameter as the additional multiplets in these model do not acquire vevs. However, the $\rho$-parameter played a key role in the construction of the GM model, as will be discussed in Chp. 7.

\subsubsection{Oblique parameters}

The oblique — or Peskin-Takeuchi — parameters [84,85] are defined as

$$
\begin{gathered}
S=\frac{4 s_{W}^{2} c_{W}^{2}}{\alpha_{\mathrm{em}} m_{Z}^{2}}\left[\Pi_{Z Z}\left(m_{Z}^{2}\right)-\Pi_{Z Z}(0)-\frac{c_{W}^{2}-s_{W}^{2}}{s_{W} c_{W}} \Pi_{Z \gamma}\left(m_{Z}^{2}\right)-\Pi_{\gamma \gamma}\left(m_{Z}^{2}\right)\right], \\
T=\frac{1}{\alpha_{\mathrm{em}}}\left[\frac{\Pi_{W W}(0)}{m_{W}^{2}}-\frac{\Pi_{Z Z}(0)}{m_{Z}^{2}}\right], \\
U=\frac{4 s_{W}^{2}}{\alpha_{\mathrm{em}}}\left[\frac{\Pi_{W W}\left(m_{W}^{2}\right)-\Pi_{W W}(0)}{m_{W}^{2}}-c_{W}^{2}\left(\frac{\Pi_{Z Z}\left(m_{Z}^{2}\right)-\Pi_{Z Z}(0)}{m_{Z}^{2}}\right)\right. \\
\left.\quad-2 s_{W} c_{W} \frac{\Pi_{Z \gamma}\left(m_{Z}^{2}\right)}{m_{Z}^{2}}-s_{W}^{2} \frac{\Pi_{\gamma \gamma}\left(m_{Z}^{2}\right)}{m_{Z}^{2}}\right],
\end{gathered}
$$

where $s_{W}=\sin \theta_{W}\left(c_{W}=\cos \theta_{W}\right)$ are the sine (cosine) of the Weinberg angle, and $\alpha_{\mathrm{em}}=e^{2} / 4 \pi$ is the electromagnetic fine structure constant. They receive contributions from new physics due to loop contributions to the gauge boson self-energies, 


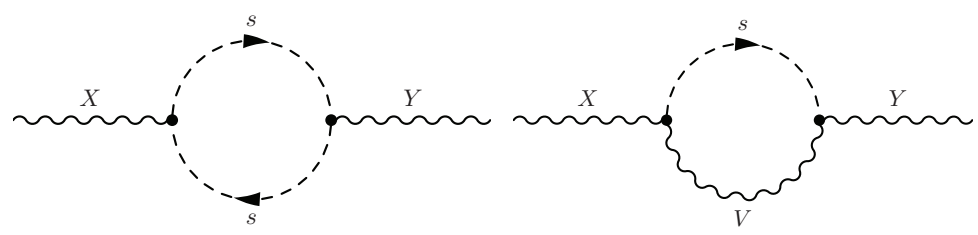

(a)

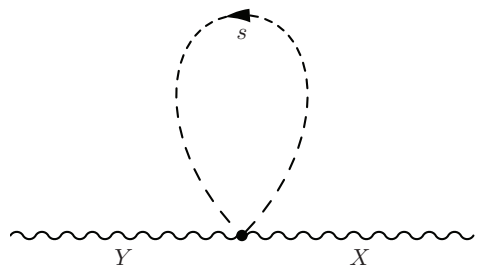

(c)

Figure 3.2: Diagrams involving scalars that contribute to oblique parameters.

$$
\Pi_{X Y}^{\mu \nu}=\Pi_{X Y}\left(p^{2}\right) g^{\mu \nu}+B_{X Y} p^{\mu} p^{\nu}
$$

which correspond to the amplitudes of the diagrams in Fig. $3.2\left(X, Y=W^{ \pm}, Z, \gamma\right)$. Here $p^{2}$ is the four-momentum of the incoming (outgoing) vector boson $X(Y)$. Note that $T$ (Eq. (3.28)) parametrizes the difference between the $W$ and $Z$ boson selfenergies at $q^{2}=0$; it is related to the $\rho$ parameter by the relation $\rho=1+\alpha_{e m} T$.

The oblique parameters of Eqs. (3.27-3.29) are defined under the assumption that the new physics is heavier than the electroweak scale (i.e. $M_{n e w}>m_{W}$ ); in the limit that any new states are a great deal heavier than the weak scale (i.e. $M_{n e w} \gg$ $\left.m_{W}\right){ }^{5}$ these definitions may be expanded about zero to find:

$$
\begin{aligned}
S & \simeq \frac{4 s_{W}^{2} c_{W}^{2}}{\alpha_{\mathrm{em}}}\left[\Pi_{Z Z}^{\prime}(0)-\frac{c_{W}^{2}-s_{W}^{2}}{s_{W} c_{W}} \Pi_{Z \gamma}^{\prime}(0)-\Pi_{\gamma \gamma}^{\prime}(0)\right], \\
T & =\frac{1}{\alpha_{\mathrm{em}}}\left[\frac{\Pi_{W W}(0)}{m_{W}^{2}}-\frac{\Pi_{Z Z}(0)}{m_{Z}^{2}}\right], \\
U & \simeq \frac{4 s_{W}^{2}}{\alpha_{\mathrm{em}}}\left[\Pi_{W W}^{\prime}(0)-c_{W}^{2} \Pi_{Z Z}^{\prime}(0)-2 s_{W} c_{W} \Pi_{Z \gamma}^{\prime}(0)-s_{W}^{2} \Pi_{\gamma \gamma}^{\prime}(0)\right] .
\end{aligned}
$$

Here the derivative is with respect to $p^{2}, \Pi_{X Y}^{\prime}=\partial \Pi_{X Y} / \partial\left(p^{2}\right)$.

\section{Self-energy diagram with two propagating scalars}

The contribution to $\Pi_{X Y}\left(p^{2}\right)$ from a diagram with two propagating scalar states $s_{1}$

\footnotetext{
${ }^{5}$ If new states arise near the electroweak scale, three additional oblique parameters called $V, X$, and $Y$ are required [86-88].
} 
and $s_{2}$ (as in Fig. 3.2a) is

$$
\Pi_{X Y}\left(p^{2}\right)=\frac{\alpha_{\mathrm{em}} C_{X s_{2} s_{1}^{*}} C_{Y s_{1} s_{2}^{*}}}{2 \pi} \int_{0}^{1} d x \Delta(\Upsilon+1-\log \Delta)
$$

where $C_{X s_{2} s_{1}^{*}}$ and $C_{Y s_{1} s_{2}^{*}}$ are the coefficients for the couplings between the two scalars and the vector bosons $X$ and $Y$ (defined as in Appendix A without a factor of $e$ ), $\Upsilon=2 / \epsilon-\gamma_{M}+\log 4 \pi+O(\epsilon), \epsilon=4-d \rightarrow 0, \gamma_{M}=0.5772$ is the Euler-Mascheroni constant, and

$$
\Delta=-x(1-x) p^{2}+x m_{1}^{2}+(1-x) m_{2}^{2} .
$$

Therefore the contribution for $p^{2} \rightarrow 0$ is

$$
\Pi_{X Y}(0)=\frac{\alpha_{\mathrm{em}} C_{X s_{2} s_{1}^{*}} C_{Y s_{1} s_{2}^{*}}}{2 \pi}\left[\frac{m_{1}^{2}+m_{2}^{2}}{2}(\Upsilon+1)-f_{2}\left(m_{1}, m_{2}\right)\right],
$$

where (assuming $m_{1} \neq m_{2}$ in the second equality)

$$
\begin{aligned}
f_{2}\left(m_{1}, m_{2}\right) & =\int_{0}^{1} d x\left(x m_{1}^{2}+(1-x) m_{2}^{2}\right) \log \left[x m_{1}^{2}+(1-x) m_{2}^{2}\right] . \\
& =-\frac{1}{4}\left(m_{1}^{2}+m_{2}^{2}\right)+\frac{1}{m_{1}^{2}-m_{2}^{2}}\left[m_{1}^{4} \log m_{1}-m_{2}^{4} \log m_{2}\right] .
\end{aligned}
$$

Similarly, the derivative with respect to $p^{2}$ at $p^{2} \rightarrow 0$ is

$$
\Pi_{X Y}^{\prime}(0)=\frac{\alpha_{\mathrm{em}} C_{X s_{2} s_{1}^{*}} C_{Y s_{1} s_{2}^{*}}}{2 \pi}\left[-\frac{\Upsilon}{6}+f_{1}\left(m_{1}, m_{2}\right)\right]
$$

where

$$
\begin{aligned}
f_{1}\left(m_{1}, m_{2}\right)= & f_{1}\left(m_{2}, m_{1}\right)=\int_{0}^{1} d x x(1-x) \log \left[x m_{1}^{2}+(1-x) m_{2}^{2}\right] \\
= & \frac{1}{36\left(m_{1}^{2}-m_{2}^{2}\right)^{3}}\left[5\left(m_{2}^{6}-m_{1}^{6}\right)+27\left(m_{1}^{4} m_{2}^{2}-m_{1}^{2} m_{2}^{4}\right)\right. \\
& \left.+12\left(m_{1}^{6}-3 m_{1}^{4} m_{2}^{2}\right) \log \left(m_{1}\right)+12\left(3 m_{1}^{2} m_{2}^{4}-m_{2}^{6}\right) \log \left(m_{2}\right)\right]
\end{aligned}
$$

for $m_{1} \neq m_{2}$. If $m_{1}=m_{2}$, then

$$
f_{1}(m, m)=\frac{1}{6} \log m^{2}, \quad \text { and } \quad f_{2}(m, m)=m^{2} \log m^{2},
$$


and the self-energies reduce to

$$
\begin{aligned}
& \Pi_{X Y}(0)=\frac{\alpha_{\mathrm{em}} C_{X s_{2} s_{1}^{*}} C_{Y s_{1} s_{2}^{*}}}{2 \pi} m^{2}\left[\Upsilon+1-\log \left[m^{2}\right]\right] \\
& \Pi_{X Y}^{\prime}(0)=\frac{\alpha_{\mathrm{em}} C_{X s_{2} s_{1}^{*}} C_{Y s_{1} s_{2}^{*}}}{12 \pi}\left[\log \left[m^{2}\right]-\Upsilon\right] .
\end{aligned}
$$

\section{Self-energy diagram with a propagating scalar and vector boson}

The contribution to $\Pi_{X Y}\left(p^{2}\right)$ from a diagram with one propagating scalar $s$ and one propagating vector boson $V$ (as in Fig. 3.2b) is

$$
\Pi_{X Y}\left(p^{2}\right)=-\alpha_{\mathrm{em}}^{2} C_{s^{*} X V} C_{s V^{*} Y^{*}} \int_{0}^{1} d x\left[\left(1-\frac{\Delta}{2 M_{V}^{2}}\right)(\Upsilon-\ln \Delta)-\frac{\Delta}{2 M_{V}^{2}}\right]
$$

where couplings $C_{s^{*} X V}$ and $C_{s V^{*} Y^{*}}$ are defined without a factor of $e^{2}, \Delta$ is the same as in Eq. (3.35); $m_{1}=M_{V}$ is the mass of the vector boson, and $m_{2}=m_{s}$ is the mass of the scalar.

Therefore the contribution for $p^{2} \rightarrow 0$ is

$\Pi_{X Y}(0)=-\alpha_{\mathrm{em}}^{2} C_{s^{*} X V} C_{s V^{*} Y^{*}}\left[(\Upsilon+1)\left(1-\frac{M_{V}^{2}+m_{s}^{2}}{4 M_{V}^{2}}\right)+f_{4}\left(M_{V}, m_{s}\right)+\frac{f_{2}\left(M_{V}, m_{s}\right)}{2 M_{V}^{2}}\right]$,

where we recall Eq. (3.37) and define

$$
f_{4}\left(m_{1}, m_{2}\right)=f_{4}\left(m_{2}, m_{1}\right)=\frac{m_{2}^{2} \ln m_{2}^{2}-m_{1}^{2} \ln m_{1}^{2}}{m_{1}^{2}-m_{2}^{2}} .
$$

The derivative at $p^{2} \rightarrow 0$ is then given by

$$
\Pi_{X Y}^{\prime}(0)=-\alpha_{\mathrm{em}}^{2} C_{s^{*} X V} C_{s V^{*} Y^{*}}\left[\frac{\Upsilon}{12 M_{V}^{2}}+f_{3}\left(m_{s}, M_{V}\right)+\frac{f_{1}\left(M_{V}, m_{s}\right)}{2 M_{V}^{2}}\right]
$$

where we recall Eq. (3.39) and define

$$
f_{3}\left(m_{1}, m_{2}\right)=f_{3}\left(m_{2}, m_{1}\right)=\frac{m_{1}^{4}-m_{2}^{4}+2 m_{1}^{2} m_{2}^{2}\left(\log m_{2}^{2}-\log m_{1}^{2}\right)}{2\left(m_{1}^{2}-m_{2}^{2}\right)^{3}} .
$$




\section{Self-energy diagram with a single propagating scalar}

The contribution to $\Pi_{X Y}\left(p^{2}\right)$ from a one-point diagram with one propagating scalar state $s$ (as in Fig. 3.2c) is

$$
\Gamma_{X Y}\left(p^{2}\right)=-\frac{\alpha_{\mathrm{em}} C_{s s^{*} X Y^{*}}}{4 \pi} m^{2}\left(\Upsilon+1-\log m^{2}\right)
$$

where the quartic coupling $C_{s s^{*} X Y^{*}}$ is defined without a factor of $e^{2}$. This is the contribution to the self-energy for $p^{2} \rightarrow 0$, as there is no $p$ dependence; i.e. $\Gamma_{X Y}\left(p^{2}\right)=$ $\Gamma_{X Y}(0)$. Because of this, $\Gamma_{X Y}^{\prime}\left(p^{2}\right)=0$.

\section{Applying constraints from oblique parameters}

$S, T$, and $U$ are calculated by considering all diagrams that contribute to the selfenergies pertinent to the oblique parameter in question, as defined by Eqs. (3.313.33). ${ }^{6}$ One will note that the results from individual diagrams still contain a divergent piece $\Upsilon$. In most models these divergences will cancel between the different scalar contributions within a multiplet. If so, a correlated 3 -observable $\chi^{2}$ constraint may be applied at 95\% confidence level (CL) using

$$
\begin{aligned}
\chi^{2}= & \frac{1}{\left(\rho_{\mathrm{ST}}^{2}+\rho_{\mathrm{TU}}^{2}+\rho_{\mathrm{SU}}^{2}-1-2 \rho_{\mathrm{ST}} \rho_{\mathrm{TU}} \rho_{\mathrm{SU}}\right)}\left[\frac{\left(\rho_{\mathrm{TU}}^{2}-1\right)\left(S-S_{\exp }\right)^{2}}{\Delta S_{\exp }^{2}}+\frac{\left(\rho_{\mathrm{SU}}^{2}-1\right)\left(T-T_{\exp }\right)^{2}}{\Delta T_{\exp }^{2}}\right. \\
& +\frac{\left(\rho_{\mathrm{ST}}^{2}-1\right)\left(U-U_{\exp }\right)^{2}}{\Delta U_{\exp }^{2}}+2\left(\rho_{\mathrm{ST}}-\rho_{\mathrm{TU}} \rho_{\mathrm{SU}}\right) \frac{\left(S-S_{\exp }\right)\left(T-T_{\exp }\right)}{\Delta S_{\exp } \Delta T_{\exp }} \\
& \left.+2\left(\rho_{\mathrm{TU}}-\rho_{\mathrm{ST}} \rho_{\mathrm{SU}}\right) \frac{\left(T-T_{\exp }\right)\left(U-U_{\exp }\right)}{\Delta T_{\exp } \Delta U_{\exp }}+2\left(\rho_{\mathrm{SU}}-\rho_{\mathrm{TU}} \rho_{\mathrm{ST}}\right) \frac{\left(S-S_{\exp }\right)\left(U-U_{\exp }\right)}{\Delta S_{\exp } \Delta U_{\exp }}\right] \\
\leq & \chi_{0.05}^{2} .
\end{aligned}
$$

\footnotetext{
${ }^{6}$ Diagrams with two distinct propagating particles in the loop must be counted twice if the particle is distinct from its anti-particle; this is to distinguish contributions of type $X \rightarrow A^{Q} B^{-Q} \rightarrow Y$ from the similar $X \rightarrow A^{-Q} B^{Q} \rightarrow Y$ contributions. Diagrams where the particles in the loop are their own anti-particles do not need to be doubled as a reversal of their direction of propagation will not result in a distinct diagram.
} 
where $\rho_{O_{i} O_{j}}$ is the correlation between observables $O_{i}, O_{j}, \delta O_{i}$ is the experimental error associated with observable $O_{i}(O=\{S, T, U\})$, and $\chi_{0.05}^{2}$ is the appropriate $\chi_{\alpha}^{2}$ value for $95 \%$ CL and the specific number of degrees of freedom,

$$
\chi_{0.05}^{2}=\left\{\begin{array}{l}
5.991 \text { for } 2 \text { degrees of freedom } \\
7.815 \text { for } 3 \text { degrees of freedom }
\end{array}\right.
$$

The combined experimental measurements for the oblique parameters are currently given by [89]

$$
\begin{array}{ll}
S_{\text {exp }}=0.05 \pm 0.11, & \rho_{\mathrm{ST}}=+0.90, \\
T_{\exp }=0.09 \pm 0.13, & \rho_{\mathrm{TU}}=-0.83, \\
U_{\exp }=0.01 \pm 0.11, & \rho_{\mathrm{SU}}=-0.59 .
\end{array}
$$

In applying constraints from oblique parameters it is often valid to assume that $U=0$ (as contributions to $U$ are negligible in many models). Under this assumption the $S$ and $T$ parameters have experimental values of $S_{\exp }^{U=0}=0.06 \pm 0.09$ and $T_{\exp }^{U=0}=$ $0.10 \pm 0.07$ with a relative correlation of $\rho_{\mathrm{ST}}^{U=0}=0.91$ [89]. In this case the $\chi^{2}$ reduces to a 2-observable constraint,

$$
\begin{aligned}
\chi^{2} & =\frac{\left(S-S_{\exp }^{U=0}\right)^{2}}{\left(\Delta S_{\exp }^{U=0}\right)^{2}\left[1-\left(\rho_{\mathrm{ST}}^{U=0}\right)^{2}\right]}-\frac{2 \rho_{\mathrm{ST}}^{U=0}\left(S-S_{\exp }^{U=0}\right)\left(T-T_{\exp }^{U=0}\right)}{\Delta S_{\exp }^{U=0} \Delta T_{\exp }^{U=0}\left[1-\left(\rho_{\mathrm{ST}}^{U=0}\right)^{2}\right]}+\frac{\left(T-T_{\exp }^{U=0}\right)^{2}}{\left(\Delta T_{\exp }^{U=0}\right)^{2}\left[1-\left(\rho_{\mathrm{ST}}^{U=0}\right)^{2}\right]} \\
& \leq \chi_{0.05}^{2} .
\end{aligned}
$$

In certain models Eqs. (3.50) and (3.53) cannot be applied due to divergences in the oblique parameters at next-to-leading order (NLO). In particular, the $T$ parameter is divergent at NLO in models which violate custodial symmetry at the loop level, such as the Georgi-Machacek model. A calculation of $T$ then requires a renormalization of the theory. In order to avoid this complication, in such cases we follow the lead 
of Ref. [6] and marginalize over $T$ in the 2-observable $\chi^{2}$; for each value of $S$ we use the value of $T$ that minimizes the 2 -observable $\chi^{2}$, resulting in a conservative bound from $S$ alone. We require that

$$
\left.\frac{\partial \chi^{2}}{\partial T}\right|_{T_{\min }}=0
$$

and so we use

$$
T \equiv T_{\min }=T_{\exp }+\rho_{\mathrm{ST}}\left(S-S_{\exp }\right) \frac{\Delta T_{\exp }}{\Delta S_{\exp }},
$$

in the 2-parameter $\chi^{2}$ formula, Eq. (3.53).

The oblique parameters will be used to constrain the parameter space of $U(1)$ and $Z_{2}$-symmetric models with large scalar multiplets in Secs. 5.3.1 and 6.3.2 respectively, as well as the Georgi-Machacek model in Sec. 7.4.1.

\subsubsection{Loop couplings of the Higgs to photons}

The Higgs boson, as a neutral particle, does not couple directly to photons. However, it may decay to two photons or $Z \gamma$ through loop processes similar to those shown in Fig. 3.3. The $h \rightarrow \gamma \gamma$ and $h \rightarrow Z \gamma$ decay widths are sensitive to charged BSM particles that can propagate in these loops, and measurements of these branching ratios can constrain the properties of such particles.

As discussed in Sec. 3.2.1, the rate at which one would experimentally observe $h \rightarrow V \gamma(V=\gamma, Z)$ as a ratio to the $\mathrm{SM}$ is given to good approximation by

$$
\mu_{V \gamma}=\frac{\sigma_{h} B R(h \rightarrow V \gamma)_{B S M}}{\sigma_{h}^{S M} B R(h \rightarrow V \gamma)_{S M}} \approx \frac{\Gamma(h \rightarrow V \gamma)_{B S M}}{\Gamma(h \rightarrow V \gamma)_{S M}}=R_{V \gamma}
$$

as long as the effects of BSM physics on the Higgs production cross section $\sigma_{h}$ and the Higgs total width $\Gamma_{\text {tot }}$ are negligible (please refer to Eq. (3.21) for a definition of the branching ratios). 


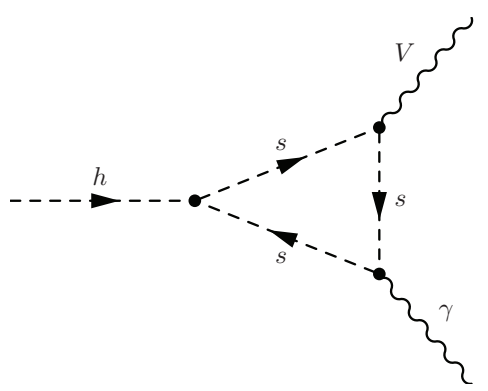

(a)

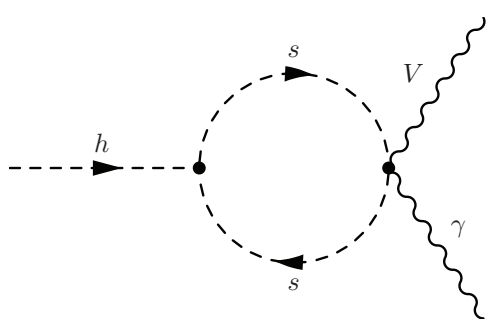

(b)

Figure 3.3: Diagrams contributing to $h \rightarrow V \gamma$.

The signal strength $\mu_{\gamma \gamma}$ has been measured by both the ATLAS and CMS detectors at the LHC $[75,90]$. To date both experiments have placed only an upper bound on the $\mu_{Z \gamma}$ signal $[75,91]$, as the signal rate for this process is much smaller than that of $h \rightarrow \gamma \gamma$ and is difficult to isolate from background at a proton-proton collider such as the LHC. The current results for both processes are listed in Table 3.1. In applying bounds to $R_{V \gamma}$ using the measured $\mu_{V \gamma}$, we conservatively require that the $R_{\gamma \gamma}$ signal predicted by our models be within two standard deviations of either measurement, and that $R_{Z \gamma}$ be below the strongest upper limit. With the current limits, this means that $h \rightarrow \gamma \gamma$ is bounded by the $2 \sigma$ result from ATLAS - i.e. $R_{\gamma \gamma} \sim$ $[0.63,1.71]$, as the CMS $2 \sigma$ bounds are within this range - while the $h \rightarrow Z \gamma$ will be constrained by the CMS limit of $R_{Z \gamma}<10$.

\section{Higgs decay to two photons}

In a scalar extension of the SM, only SM fermions $f$, the SM $W$ boson, and any new charged scalars $(s)$ may propagate in the loop of the Higgs $h \rightarrow \gamma \gamma$ decay. In this case the prediction for the total width of Higgs decay to $\gamma \gamma$ - where both outgoing 


\begin{tabular}{ccc}
\hline \hline Observable & ATLAS & CMS \\
\hline$\mu_{\gamma \gamma}$ & $1.17_{-0.27}^{+0.27}[75]$ & $1.14_{-0.23}^{+0.26}[90]$ \\
$\mu_{Z \gamma}$ & $<11[75]$ & $<10[91]$ \\
\hline \hline
\end{tabular}

Table 3.1: 2014 measurements of the signal strengths for $h \rightarrow \gamma \gamma$ and $h \rightarrow Z \gamma$ relative to the SM predictions. For $h \rightarrow Z \gamma$ we quote the $95 \%$ confidence level upper bounds for $M_{h}=125 \mathrm{GeV}$.

photons are on-shell — is described by

$$
\Gamma(h \rightarrow \gamma \gamma)=\frac{m_{h}^{3}}{64 \pi}\left|A_{t o t}^{h \gamma \gamma}\right|^{2}
$$

where the form factor

$$
A_{t o t}^{h \gamma \gamma}=\frac{\alpha_{\mathrm{em}} g}{4 \pi m_{W}}\left(\kappa_{f} A_{F}^{h \gamma \gamma}+\kappa_{W} A_{W}^{h \gamma \gamma}+A_{S}^{h \gamma \gamma}\right)
$$

is related to the matrix element by $\mathcal{M}_{\gamma \gamma}=A_{t o t}^{\gamma \gamma}\left(g^{\mu \nu} k \cdot q-k^{\mu} q^{\nu}\right) \varepsilon_{\mu}^{*}(k) \varepsilon_{\nu}^{*}(q)$, where $k_{\nu}$ and $q_{\mu}$ are the outgoing four-momenta of the photons [92]. Here $A_{F, W, S}^{\gamma \gamma}$ respectively correspond to contributions from any charged fermions, vector bosons, or scalars that are permitted to propagate in the loop. The functions $A_{F, W, S}^{h \gamma \gamma}$ are defined as [92]

$$
\begin{aligned}
& A_{S}^{h \gamma \gamma}=\sum_{s} Q_{s}^{2} C_{h s s^{*}} \frac{v}{2 m_{s}^{2}} \tau_{s}\left[1-\tau_{s} f\left(\tau_{s}\right)\right], \\
& A_{W}^{h \gamma \gamma}=2+3 \tau_{W}+3 \tau_{W}\left(2-\tau_{W}\right) f\left(\tau_{W}\right), \\
& A_{F}^{h \gamma \gamma}=-2 \sum_{f} N_{c, f} Q_{f}^{2} \tau_{f}\left[1+\left(1-\tau_{f}\right) f\left(\tau_{f}\right)\right] .
\end{aligned}
$$

Here $Q$ denotes electric charge (in units of $e$ ), $N_{c, f}$ is the color multiplicity of fermion $f, s$ and $f$ respectively run over all charged scalars and fermions, $C_{h s s^{*}}$ is the coupling between the Higgs $h$ and a scalar anti-scalar pair of species $s$, and

$$
\tau_{k}=\frac{4 m_{k}^{2}}{m_{h}^{2}},
$$


is a ratio involving the parent scalar mass $m_{h}$ and the mass $m_{k}$ of the propagating particle $k$. The function $f(\tau)$ is given by

$$
f(\tau)=\left\{\begin{array}{cc}
{\left[\arcsin \left(\sqrt{\frac{1}{\tau}}\right)\right]^{2}} & \text { if } \tau \geq 1, \\
-\frac{1}{4}\left[\ln \left(\frac{\eta_{+}}{\eta_{-}}\right)-i \pi\right]^{2} & \text { if } \tau<1,
\end{array}\right.
$$

where $\eta_{ \pm}=1 \pm \sqrt{1-\tau}$ [92]. We can see from these formulas that in a BSM model which only contains new scalar states, the $h \rightarrow \gamma \gamma$ decay rate will constrain the scalar masses $m_{s}$ and the scalar couplings to the Higgs, $C_{h s s^{*}}$.

The factors $\kappa_{f}$ and $\kappa_{W}$ in Eq. (3.58) are ratios that describe any differences in the couplings between the Higgs boson $h$ and the SM fermions and $W$ boson. Changes in these couplings may be due to differences in the particle states themselves (e.g. from mixing with BSM particles), differences in the Higgs mechanism, or due to loop-corrections to the coefficients from new particles. Eq. (3.58) assumes that $\kappa_{f}$ is the same for all species of fermions $f .{ }^{7}$ This is the case for all of the models we consider in this thesis, as all of the fermions and gauge bosons are SM particles and fermion interactions with scalars are mediated by a single electroweak scalar doublet. As a result, any differences in the Higgs couplings arise from differences in the Higgs mechanism such that couplings to fermions $h \bar{f} f$ are all be affected by a common factor $\kappa_{f}$ and tree-level gauge boson couplings $h V V$ are all altered by a common factor $\kappa_{V} \cdot{ }^{8}$ These factors are defined as $[73,74]$

$$
\kappa_{f}=\frac{C_{h \bar{f} f}^{\mathrm{BSM}}}{C_{h \bar{f} f}^{\mathrm{SM}}} \quad \text { and } \quad \kappa_{V}=\frac{C_{h V V}^{\mathrm{BSM}}}{C_{h V V}^{\mathrm{SM}}},
$$

in accordance with the notation used by collider experiments [93]. Therefore the SM prediction for the $h \rightarrow \gamma \gamma$ decay width is given by Eqs. (3.57-3.61) with $A_{S}=0$, $\kappa_{f}=\kappa_{W}=1$.

\footnotetext{
${ }^{7}$ In models where $\kappa_{f}$ is different for different species of fermion $f$, this factor would have to be included under the sum of Eq. (3.61).

${ }^{8}$ Loop-effects to these couplings will be neglected for our purposes.
} 
As the fermionic contribution $A_{F}$ is proportional to the fermion mass through the ratio $\tau_{f}$, this contribution will be dominated by the top quark. In practice, it is often convenient to neglect contributions from the light quarks,

$$
A_{F}^{h \gamma \gamma} \simeq-\frac{8}{3} \tau_{t}\left[1+\left(1-\tau_{t}\right) f\left(\tau_{t}\right)\right],
$$

where $\tau_{t}=4 m_{t}^{2} / m_{h}^{2}$ as defined by Eq. (3.62) and $m_{t}$ is the mass of the top quark. We will employ this approximation throughout the work that follows.

The $h \rightarrow \gamma \gamma$ coupling may also be expressed as a ratio to the SM coupling in analogy to $\kappa_{V, f}$ - by taking a ratio of the amplitudes $A_{F, W, S}$ defined in Eqs. (3.593.61). As the SM contribution is given by $A_{W}^{h \gamma \gamma}+A_{F}^{h \gamma \gamma}$, the coupling ratio is

$$
\kappa_{\gamma} \equiv \frac{A_{t o t}^{h \gamma \gamma, \mathrm{BSM}}}{A_{t o t}^{h \gamma \gamma, \mathrm{SM}}}=\frac{\kappa_{W} A_{W}^{h \gamma \gamma}+\kappa_{f} A_{F}^{h \gamma \gamma}+A_{S}^{h \gamma \gamma}}{A_{W}^{h \gamma \gamma}+A_{F}^{h \gamma \gamma}} .
$$

One will note that there are two kinds of contributions that may alter this coupling. The first category consists of any differences in the SM fermion or vector boson couplings, which are parametrized by $\kappa_{f}$ or $\kappa_{V}$. The second consists of contributions from new particles propagating in the loop, which will be new scalar contributions to $A_{S}$ in the models we consider. In order to separate these two BSM influences on $h \rightarrow \gamma \gamma$, it will also be useful to define

$$
\Delta \kappa_{\gamma}=\frac{A_{S}^{h \gamma \gamma}}{A_{W}^{h \gamma \gamma}+A_{F}^{h \gamma \gamma}},
$$

which includes only the effects from new scalar particles propagating in the loop.

\section{Higgs decay to a photon and a $Z$ boson}

The prediction for the $h \rightarrow Z \gamma$ decay width is similar in structure to that of $h \rightarrow \gamma \gamma$. However, it is complicated by the fact that one of the outgoing bosons is now massive, 
so certain simplifications (i.e. $m_{\gamma}^{2}=0$ ) no longer apply in the loop diagram calculation. The decay width therefore becomes,

$$
\Gamma(h \rightarrow Z \gamma)=\frac{m_{h}^{3}}{32 \pi}\left|A_{t o t}^{h Z \gamma}\right|^{2}\left(1-\frac{m_{Z}^{2}}{m_{h}^{2}}\right)^{3},
$$

where the form factor

$$
A_{t o t}^{h Z \gamma}=-\frac{\alpha_{\mathrm{em}} g}{4 \pi m_{W}}\left(\kappa_{f} A_{F}^{h Z \gamma}+\kappa_{W} A_{W}^{h Z \gamma}+A_{S}^{h Z \gamma}\right)
$$

is related to the matrix element $\mathcal{M}_{Z \gamma}$ in an analogous way to the $\gamma \gamma$ case (see below Eq. (3.58)). Here the contributions from scalars $S$, SM fermions $F$, and the SM $W$ boson are respectively given by

$$
\begin{aligned}
A_{S}^{h Z \gamma}= & \frac{2 m_{W}}{g} \frac{C_{h s s^{*}} C_{Z s s^{*}} Q}{m_{s}^{2}} I_{1}\left(\frac{4 m_{s}^{2}}{M_{h}^{2}}, \frac{4 m_{s}^{2}}{m_{Z}^{2}}\right), \\
A_{W}^{h Z_{\gamma}}= & -\cot \theta_{W}\left\{4\left(3-\tan ^{2} \theta_{W}\right) I_{2}\left(\tau_{W}, \lambda_{W}\right)\right. \\
& \left.+\left[\left(1+\frac{2}{\tau_{W}}\right) \tan ^{2} \theta_{W}-\left(5+\frac{2}{\tau_{W}}\right)\right] I_{1}\left(\tau_{W}, \lambda_{W}\right)\right\}, \\
A_{F}^{h Z \gamma}= & \sum_{f} N_{c f} \frac{-2 Q_{f}\left(T_{f}^{3 L}-2 Q_{f} \sin ^{2} \theta_{W}\right)}{\sin \theta_{W} \cos \theta_{W}}\left[I_{1}\left(\tau_{f}, \lambda_{f}\right)-I_{2}\left(\tau_{f}, \lambda_{f}\right)\right], \\
\simeq & \frac{2\left(8 / 3 \sin ^{2} \theta_{W}-1\right)}{\sin \theta_{W} \cos \theta_{W}}\left[I_{1}\left(\tau_{t}, \lambda_{t}\right)-I_{2}\left(\tau_{t}, \lambda_{t}\right)\right] .
\end{aligned}
$$

Here $Q$ and $T^{3}$ denote electric charge and third component of weak isospin respectively, $C_{h s s^{*}}$ and $C_{Z s s^{*}}$ are the couplings of a Higgs or $Z$ to a scalar-anti-scalar pair (without a factor of $e$ ), and we make use of $g^{2} / m_{W}^{2}=4 / v^{2}$. In Eq. (3.73) we keep only the contribution from the top quark. We define $\tau_{k}$ and $f(\tau)$ according to Eqs. (3.62) and (3.63) respectively, $\lambda_{k}=4 m_{k}^{2} / m_{Z}^{2}$ for a propagating particle $k$, and

$$
g(\tau)=\left\{\begin{array}{cl}
\sqrt{\tau-1} \arcsin \left(\sqrt{\frac{1}{\tau}}\right) & \text { if } \tau \geq 1, \\
\frac{1}{2} \sqrt{1-\tau}\left[\ln \left(\frac{\eta_{+}}{\eta_{-}}\right)-i \pi\right] & \text { if } \tau<1,
\end{array}\right.
$$




$$
\begin{aligned}
& I_{1}(a, b)=\frac{a b}{2(a-b)}+\frac{a^{2} b^{2}}{2(a-b)^{2}}[f(a)-f(b)]+\frac{a^{2} b}{(a-b)^{2}}[g(a)-g(b)], \\
& I_{2}(a, b)=-\frac{a b}{2(a-b)}[f(a)-f(b)] .
\end{aligned}
$$

Note that the scalar contribution $A_{S}$ considers only loops that involve a single type of scalar particle, as mixed $h \rightarrow Z \gamma$ loops that include more than one scalar species are not relevant to the models considered in this work. ${ }^{9}$ One will further observe that Eq. (3.68) differs by a factor of 2 when compared with the corresponding $h \rightarrow \gamma \gamma$ formula of Eq. (3.57). This is due to the additional symmetry factor for identical particles in the $\gamma \gamma$ case. If the $m_{Z} \rightarrow 0$ limit is taken and the couplings involving $Z$ are replaced by the corresponding photon couplings, it can be shown that $A_{F, W, S}^{Z \gamma} \rightarrow$ $-A_{F, W, S}^{\gamma \gamma}$ and $\Gamma(h \rightarrow Z \gamma) \rightarrow 2 \Gamma(h \rightarrow \gamma \gamma)$, as one would expect [92].

As in the $\gamma \gamma$ case, the ratio $\kappa_{Z \gamma}$ of the $h \rightarrow Z \gamma$ coupling in a BSM model compared to the SM is given by

$$
\kappa_{Z \gamma}=\frac{\kappa_{W} A_{W}^{h Z \gamma}+\kappa_{f} A_{F}^{h Z \gamma}+A_{S}^{h Z \gamma}}{A_{W}^{h Z \gamma}+A_{f}^{h Z \gamma}},
$$

and we also define

$$
\Delta \kappa_{Z \gamma}=\frac{A_{S}^{h Z_{\gamma}}}{A_{W}^{h Z \gamma}+A_{f}^{h Z \gamma}},
$$

to isolate the effect from loop-propagating scalar particles.

\subsubsection{Interactions of charged scalars with fermions}

If the BSM scalars couple to fermions, they can also contribute to a variety of fermionic processes at loop-level. Four such processes — which have been extensively studied

\footnotetext{
${ }^{9}$ However, in Chp. 7 we will consider the decay to $Z \gamma$ of a non-SM-like neutral scalar which does involve loops with mixed species of scalars.
} 
at various colliders - are $Z \rightarrow b \bar{b}$ decay, $B_{s, d}^{0}-\bar{B}_{s, d}^{0}$ meson mixing, $B_{s, d}^{0} \rightarrow \mu^{+} \mu^{-}$decay, and $b \rightarrow s \gamma$. Figures 3.4-3.7 illustrate typical contributions.

In all four processes, singly-charged BSM scalar particles may contribute by effectively replacing $W$ bosons in the corresponding SM processes. In the case of both $Z \rightarrow b \bar{b}$ and $B_{s, d}^{0} \rightarrow \mu^{+} \mu^{-}$— which both proceed through similar penguin diagrams - neutral BSM scalars may also participate. However, contributions from neutral scalars are usually very small compared to those from singly-charged scalars. This is because the charged scalar processes can involve top quarks, and will be dominated by the large top quark mass. All processes involving lighter fermions will be suppressed by their relatively small masses. Furthermore, if the scalar couplings to fermions arise from a single electroweak scalar doublet, the scalar couplings to light fermions cannot be enhanced without similarly enhancing the top quark coupling (which will eventually hit a unitarity bound). In this situation, the contributions involving the top quark will always dominate these processes.

This is the case for the GM model, the only model we consider that may be constrained by these processes (see Sec. 7.4.2). The BSM scalars in the other models we consider do not couple to fermions.

\section{$Z$-decay to $b \bar{b}$}

The observable $R_{b}$, defined as

$$
R_{b}=\frac{\Gamma(Z \rightarrow b \bar{b})}{\Gamma(Z \rightarrow \text { hadrons })},
$$

is sensitive to new physics contributions to the left- and right-handed $Z b \bar{b}$ couplings. Following Ref. [94], the correction to $R_{b}^{\mathrm{SM}}$ is

$$
\delta R_{b}=-0.7785 \delta g_{L}+0.1409 \delta g_{R}
$$




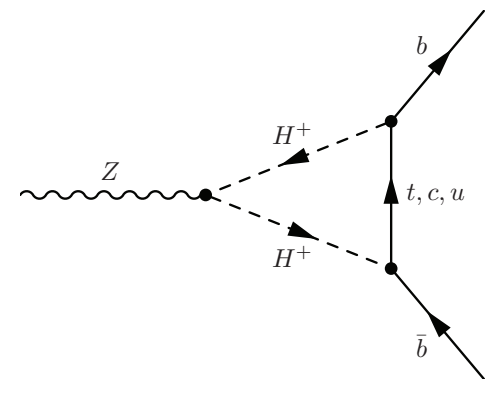

(a)

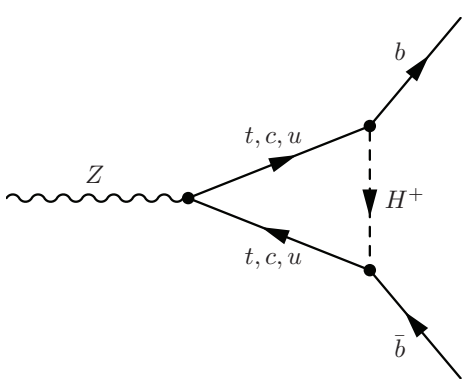

(b)

Figure 3.4: Sample diagrams of charged scalar contributions to $R_{b}$.

where $\delta g_{L, R}$ are the scalar loop contributions to the left-handed and right-handed $Z b \bar{b}$ couplings respectively. ${ }^{10}$ The particular form of the $\delta g_{L, R}$ will depend on the model under consideration. In general both neutral and singly charged scalars can contribute to these $Z b \bar{b}$ couplings, respectively corresponding to propagating down-type or uptype quarks in Fig. 3.4. We will take the uncertainty on the BSM prediction for $R_{b}$ to be the same as that of the SM prediction.

The measured value of $R_{b}$ is [89]

$$
R_{b}^{\exp }=0.21629 \pm 0.00066
$$

which is currently less than $0.8 \sigma$ from the SM prediction [89] of

$$
R_{b}^{\mathrm{SM}}=0.21577 \pm 0.00011
$$

Therefore the $2 \sigma$ bound of

$$
R_{b}^{\mathrm{BSM}}=R_{b}^{\mathrm{SM}}+\delta R_{b}^{\mathrm{BSM}}>0.21495
$$

will constrain the masses and coupling parameters of any scalars propagating in the loop, where we have combined the experimental and SM prediction uncertainties in

\footnotetext{
${ }^{10}$ The coefficient -0.7785 depends on the $Z b \bar{b}$ couplings and the bottom quark mass. Updated values of these quantities have a very small effect on the coefficient. For example, the change in the coefficient is $0.1 \%$ when using more recent values from Ref. [95].
} 
quadrature. Substantial improvement in the $R_{b}$ experimental measurement is unlikely in the near future, as this would require better $Z$-pole measurements using a nextgeneration $e^{+} e^{-}$collider like the International Linear Collider (ILC) with the GigaZ option [96].

\section{B-meson mixing}

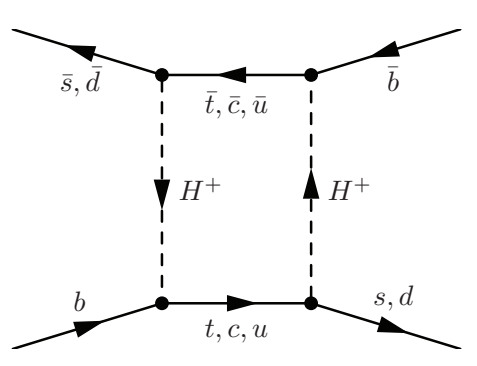

(a)

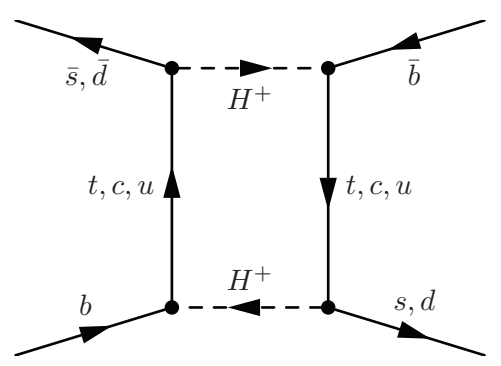

(b)

Figure 3.5: Sample diagrams of charged scalar contributions to $B_{s, d}^{0}-\bar{B}_{s, d}^{0}$.

$B_{s}^{0}$ and $B_{d}^{0}$ mesons are known to oscillate between their particle and antiparticle states $\left(B_{s, d}^{0}-\bar{B}_{s, d}^{0}\right)$ via box diagrams. While in the SM this oscillation is mediated purely by $W$ gauge bosons, singly-charged BSM scalars can also contribute to this process by replacing one or both of the propagating $W$ bosons, as shown in Fig. 3.5. The oscillation frequency is determined by the mass splitting between the particle and anti-particle states, $\Delta m_{B_{s, d}}$, and is given at next-to-leading order by $[97,98]$

$$
\Delta m_{B_{q}}=\frac{G_{F}^{2} m_{t}^{2}}{24 \pi^{2}}\left|V_{t q}^{*} V_{t b}\right|^{2} f_{B_{q}}^{2} B_{B_{q}} m_{B_{q}} \eta_{b} I
$$

Here $q=s, d, \eta_{b}$ is a QCD correction factor, $f_{B_{q}}$ is the weak decay constant, $B_{B_{q}}$ is the bag parameter, $m_{B_{q}}$ is the meson mass, and $V_{i j}$ are the relevant CKM matrix elements. The function $I$ is a linear combination of Inami-Lim loop functions that 
is model-dependent [99]. For example, in the SM — where only the $W$ boson may mediate the process - the function $I$ is given by

$$
I_{S M}=I_{W W}\left(\frac{\bar{m}_{t}^{2}\left(\mu_{t}\right)}{m_{W}^{2}}\right) \text {, where } I_{W W}(x)=1+\frac{9}{1-x}-\frac{6}{(1-x)^{2}}-\frac{6 x^{2} \ln (x)}{(1-x)^{3}}
$$

and the $\overline{\mathrm{MS}}$ top quark mass $\bar{m}_{t}\left(\mu_{t}\right)$ is renormalized at the scale of the top pole mass $\mu_{t}=m_{t}$. However, in BSM models new singly charged particles can contribute additional terms to $I$.

If the new particle states in a BSM model do not substantially contribute to QCD corrections - as is the case with electroweak scalars - the BSM model prediction for $\Delta m_{B_{q}}$ can be calculated as a ratio to the Standard Model result,

$$
\Delta m_{B_{q}}^{\mathrm{BSM}}=\Delta m_{B_{q}}^{S M} \cdot R_{\Delta m}^{\mathrm{BSM}} \quad \text { where } \quad R_{\Delta m}^{\mathrm{BSM}}=\frac{I_{\mathrm{BSM}}}{I_{\mathrm{SM}}}
$$

The uncertainty on this prediction can similarly be obtained by scaling the SM prediction uncertainty by $R_{\Delta m}^{\mathrm{BSM}}$.

Currently the strongest constraints for this observable are given by $B_{s}^{0}$ mixing, as the uncertainties on both the experimental measurement and SM prediction are tighter than those for $B_{d}^{0}$ mixing. The measured value for the $B_{s}^{0}-\bar{B}_{s}^{0}$ mass difference is given by [43]

$$
\Delta m_{B_{s}}^{\exp }=17.719 \pm 0.036(\text { stat }) \pm 0.023 \text { (syst) } \mathrm{ps}^{-1} \text {. }
$$

The largest uncertainty in the SM prediction comes from the lattice QCD calculation of $f_{B_{s}} B_{B_{s}}^{1 / 2}$. Using a CKMfitter [100,101] average of several lattice results based on separate extractions of $f_{B_{s}}$ and $B_{B_{s}}$, Ref. [102] obtains the SM prediction of $\Delta m_{B_{s}}^{\mathrm{SM}}=17.3 \pm 1.5 \mathrm{ps}^{-1}$. However, a preliminary lattice calculation of the product $f_{B_{s}} B_{B_{s}}^{1 / 2}$ from the Fermilab/MILC collaboration [103] yields a larger uncertainty and 
considerably different central value, leading to $\Delta m_{B_{s}}^{\mathrm{SM}}=21.7 \pm 2.6 \mathrm{ps}^{-1}$ [102]. For our numerical results we use the CKMfitter central value but take the more conservative uncertainty as advocated in Ref. [104],

$$
\Delta m_{B_{s}}^{\mathrm{SM}}=17.3 \pm 2.6 \mathrm{ps}^{-1} .
$$

Eqs. (3.87)-(3.88) can be translated to give an experimental measurement of

$$
R_{\Delta m}^{\exp } \equiv \frac{\Delta m_{B_{s}}^{\exp }}{\Delta m_{B_{s}}^{\mathrm{SM}}}=1.02 \pm 0.15
$$

as a ratio to the SM prediction. The SM prediction $R_{\Delta m}^{\mathrm{SM}}=1$ is thus only $0.13 \sigma$ below the measured value. Models with BSM scalars contributing to this process are then constrained by a $2 \sigma$ upper bound of

$$
R_{\Delta m}^{\mathrm{BSM}} \equiv \frac{\Delta m_{B_{s}}^{\mathrm{BSM}}}{\Delta m_{B_{s}}^{\mathrm{SM}}} \leq 1.46,
$$

where we have combined the experimental and BSM prediction uncertainties in quadrature. An improvement in the precision of this constraint relies on an improved lattice determination of $f_{B_{s}} B_{B_{s}}^{1 / 2}$.

\section{B meson decay to muons}

Deviations due to new particles may also be measurable in the time-integrated branching ratio of $B$-mesons to muons, $\overline{\mathrm{BR}}\left(B_{s}^{0} \rightarrow \mu^{+} \mu^{-}\right)$, as shown in Fig. 3.6. To leading order, this decay is given by [105]

$$
\overline{\mathrm{BR}}\left(B_{s}^{0} \rightarrow \mu^{+} \mu^{-}\right)_{\mathrm{BSM}}=\left[\frac{1+A_{\Delta \Gamma}^{\mu \mu} y_{q}}{1+y_{q}}\right]\left[|P|^{2}+|S|^{2}\right] \overline{\mathrm{BR}}\left(B_{s}^{0} \rightarrow \mu^{+} \mu^{-}\right)_{\mathrm{SM}},
$$

where $A_{\Delta \Gamma}^{\mu \mu}$ and $y_{q}$ are factors that account for the time-integration of the process, ${ }^{11}$

\footnotetext{
${ }^{11}$ Time integration is required because the oscillation of the $B$ meson over time will affect the decay rate, since the $\mathrm{CP}$-odd combination has a sightly different width than the $\mathrm{CP}$-even combination.
} 


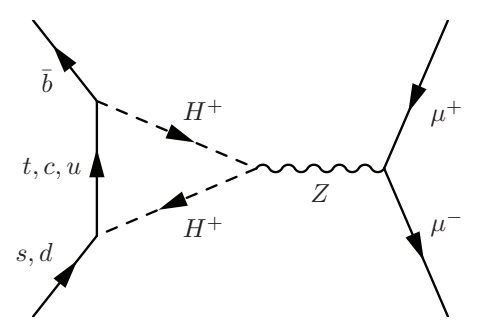

(a)

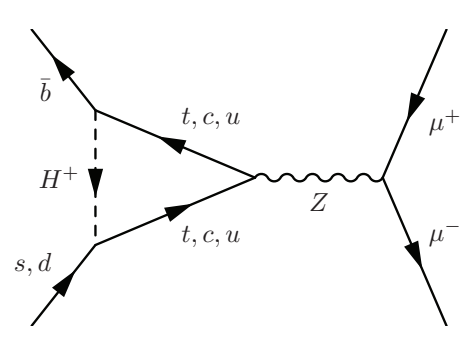

(b)

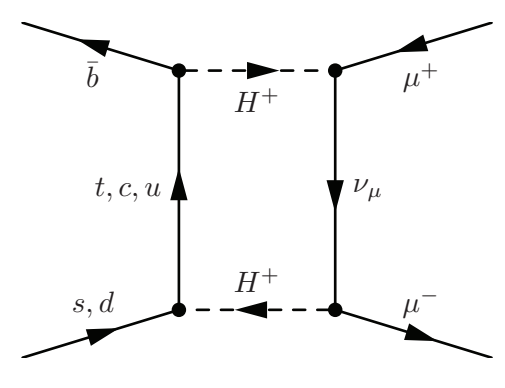

(c)

Figure 3.6: Sample diagrams of charged scalar contributions to $B_{s, d}^{0} \rightarrow \mu^{+} \mu^{-}$.

The contributions $P$ and $S$ are defined as

$$
\begin{aligned}
& P=\frac{C_{10}}{C_{10}^{\mathrm{SM}}}+\frac{m_{B_{q}}^{2}}{2 m_{W}^{2}}\left(\frac{m_{b}}{m_{b}+m_{q}}\right) \frac{C_{P}-C_{P}^{\mathrm{SM}}}{C_{10}^{\mathrm{SM}}}, \\
& S=\sqrt{1-\frac{4 m_{\mu}^{2}}{m_{B_{q}}^{2}} \frac{m_{B_{q}}^{2}}{2 m_{W}^{2}}\left(\frac{m_{b}}{m_{b}+m_{q}}\right) \frac{C_{S}-C_{S}^{\mathrm{SM}}}{C_{10}^{\mathrm{SM}}}},
\end{aligned}
$$

where $m_{B_{q}}$ is the mass of $B_{q}^{0}$. The Wilson coefficients $C_{P}$ and $C_{S}$ respectively account for scalar and pseudoscalar contributions to the process. If the BSM model does not introduce new sources of $\mathrm{CP}$ violation and the differences $C_{P, S}-C_{P, S}^{\mathrm{SM}}$ between the SM and BSM model may be neglected, the branching ratio reduces to

$$
\begin{gathered}
\overline{\mathrm{BR}}\left(B_{s}^{0} \rightarrow \mu^{+} \mu^{-}\right)_{\mathrm{BSM}}=\overline{\mathrm{BR}}\left(B_{s}^{0} \rightarrow \mu^{+} \mu^{-}\right)_{\mathrm{SM}} \cdot R_{B_{s}^{0} \rightarrow \mu^{+} \mu^{-}} \\
\text {where } R_{B_{s}^{0} \rightarrow \mu^{+} \mu^{-}}=\left|\frac{C_{10}^{\mathrm{BSM}}}{C_{10}^{\mathrm{SM}}}\right|^{2} .
\end{gathered}
$$

The uncertainty on this prediction can be obtained by scaling the SM prediction uncertainty by $R_{B_{s}^{0} \rightarrow \mu^{+} \mu^{-}}$. The SM Wilson coefficient can be approximated by [105]

$$
C_{10}^{\mathrm{SM}}=-0.9380\left[\frac{m_{t}}{173.1 \mathrm{GeV}}\right]^{1.53}\left[\frac{\alpha_{s}\left(m_{Z}\right)}{0.1184}\right]^{-0.09} .
$$

where $m_{t}$ is the top quark pole mass and the strong coupling constant $\alpha_{s}\left(m_{Z}\right)=$ 0.11856 [43]. The expression for $C_{10}^{\mathrm{SM}}$ includes next-to-leading order (NLO) elec- 
troweak and QED corrections, as well as NLO and next-to-next-to-leading order (NNLO) QCD corrections.

The current experimental measurement of $\overline{\mathrm{BR}}\left(B_{s}^{0} \rightarrow \mu^{+} \mu^{-}\right)$from a combination of CMS and LHCb results is [106]

$$
\overline{\mathrm{BR}}\left(B_{s}^{0} \rightarrow \mu^{+} \mu^{-}\right)_{\exp }=(2.9 \pm 0.7) \times 10^{-9} .
$$

The SM prediction is given in Ref. [105] as

$$
\overline{\mathrm{BR}}\left(B_{s}^{0} \rightarrow \mu^{+} \mu^{-}\right)_{\mathrm{SM}}=(3.67 \pm 0.25) \times 10^{-9} .
$$

This number differs slightly from the result in Ref. [107], upon which it is based, due to the use of a slightly different central value and more conservative uncertainty on the top quark mass. These yield an experimental ratio of

$$
\bar{R}_{B_{s}^{0} \rightarrow \mu^{+} \mu^{-}}^{\exp } \equiv \frac{\overline{\mathrm{BR}}\left(B_{s}^{0} \rightarrow \mu^{+} \mu^{-}\right)_{\exp }}{\overline{\mathrm{BR}}\left(B_{s}^{0} \rightarrow \mu^{+} \mu^{-}\right)_{\mathrm{SM}}}=0.79 \pm 0.20
$$

In particular, the SM prediction, $\bar{R}_{s \mu}^{\mathrm{SM}}=1$, is $1.0 \sigma$ above the current experimental value. The $2 \sigma$ constraint relative to the experimental central value yields an upper bound of

$$
\bar{R}_{B_{s}^{0} \rightarrow \mu^{+} \mu^{-}}^{\mathrm{BSM}}=\frac{\overline{\mathrm{BR}}\left(B_{s}^{0} \rightarrow \mu^{+} \mu^{-}\right)_{\mathrm{BSM}}}{\overline{\mathrm{BR}}\left(B_{s}^{0} \rightarrow \mu^{+} \mu^{-}\right)_{\mathrm{SM}}} \leq 1.21 .
$$

The experimental and BSM prediction uncertainties are combined in quadrature.

The current uncertainty on $\bar{R}_{B_{s}^{0} \rightarrow \mu^{+} \mu^{-}}$is dominated by the experimental statistical uncertainty. This has the potential to be significantly reduced in the near future as more data is collected at the LHC. In particular, the upgraded LHCb experiment is expected to measure $\overline{\mathrm{BR}}\left(B_{s}^{0} \rightarrow \mu^{+} \mu^{-}\right)$with an ultimate experimental uncertainty of better than $10 \%$ with $50 \mathrm{fb}^{-1}$ of data [108], which corresponds to about ten years of LHC running. 


\section{Radiative bottom quark decay, $b \rightarrow s \gamma$}

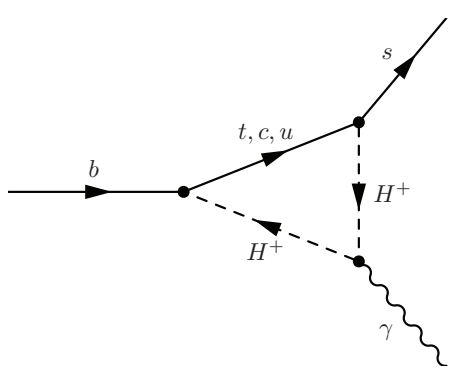

(a)

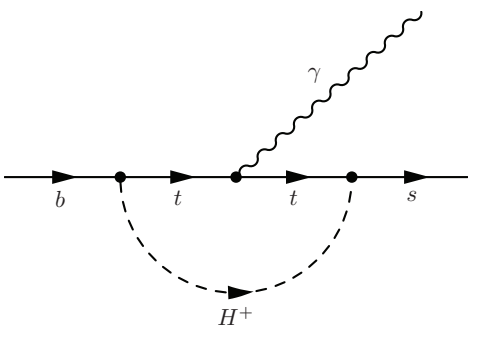

(b)

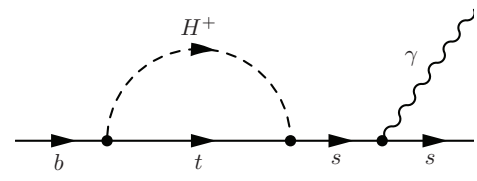

(c)

Figure 3.7: Sample diagrams of charged scalar contributions to $b \rightarrow s \gamma$.

The quark-level process $b \rightarrow s \gamma$ may receive contributions from singly-charged BSM scalars, as shown in Fig. 3.7. It is predominantly measured through the full $\bar{B} \rightarrow X_{s} \gamma$ decay. To leading order, the branching ratio for $\bar{B} \rightarrow X_{s} \gamma$ is given by

$$
B R\left(\bar{B} \rightarrow X_{s} \gamma\right)=\frac{\left|V_{t s}^{*} V_{t b}\right|^{2}}{\left|V_{c b}\right|^{2}} \frac{6 \alpha_{e m}}{\pi g\left(m_{c}^{2} / m_{b}^{2}\right)}\left|C_{7}^{(0) e f f}\left(\mu_{b}\right)\right|^{2} \times B R\left(\bar{B} \rightarrow X_{c} e \bar{\nu}_{e}\right)
$$

where $V_{i j}$ are CKM matrix elements, $\alpha_{e m}$ is the electromagnetic fine structure constant, $C_{7}^{(0) e f f}\left(\mu_{b}\right)$ is an effective Wilson coefficient, and $g(x)$ is a phase space factor [109]. As before, contributions from electroweak scalars will not substantially alter QCD processes and so their dominant contribution will be to the Wilson coefficient $C_{7}$. The decay can then be predicted using a ratio to the SM,

$$
\begin{gathered}
B R\left(\bar{B} \rightarrow X_{s} \gamma\right)_{\mathrm{BSM}}=B R\left(\bar{B} \rightarrow X_{s} \gamma\right)_{\mathrm{SM}} \cdot R_{b \rightarrow s \gamma}^{\mathrm{BSM}}, \\
\text { where } R_{b \rightarrow s \gamma}^{\mathrm{BSM}}=\left|\frac{C_{7, \mathrm{BSM}}^{(0) e f f}\left(\mu_{b}\right)}{C_{7, \mathrm{SM}}^{(0) e f f}\left(\mu_{b}\right)}\right|^{2} .
\end{gathered}
$$

The error on this BSM prediction may similarly be obtained by scaling the SM prediction uncertainty by $R_{b \rightarrow s \gamma}^{\mathrm{BSM}}$. An effective coefficient $C_{7}^{(0) e f f}\left(\mu_{b}\right)$ defined at renormalization scale $\mu_{b} \sim m_{b}$ is related to the coefficients $C_{7}^{(0)}\left(\mu_{W}\right)$ and $C_{8}^{(0)}\left(\mu_{W}\right)$ at the 
higher renormalization scale $\mu_{W} \sim m_{W}$ by the following evolution:

$$
C_{7}^{(0) e f f}\left(\mu_{b}\right)=\eta^{\frac{16}{23}} C_{7}^{(0)}\left(\mu_{W}\right)+\frac{8}{3}\left(\eta^{\frac{14}{23}}-\eta^{\frac{16}{23}}\right) C_{8}^{(0)}\left(\mu_{W}\right)+\sum_{i=1}^{8} h_{i} \eta^{a_{i}}
$$

The values of $h_{i}$ and $a_{i}$ may be found in Table 1 of Ref. [109], and $\eta=\alpha_{s}\left(\mu_{W}\right) / \alpha_{s}\left(\mu_{b}\right)$. The formula for $\alpha(\mu)$ at different renormalization scales $\mu$ can be found in Eq. (B.6). At the $\mu_{W}$ scale, the Wilson coefficients are given to LO in the SM by $C_{i, S M}^{(0)}\left(\mu_{W}\right)=$ $F_{i}^{(1)}\left(x_{W}\right)(i=7,8)$, where

$$
\begin{aligned}
& F_{7}^{(1)}(x)=\frac{x^{2}(3 x-2)}{4(x-1)^{4}} \ln x+\frac{-8 x^{3}-5 x^{2}+7 x}{24(x-1)^{3}} \\
& F_{8}^{(1)}(x)=\frac{-3 x^{2}}{4(x-1)^{4}} \ln x+\frac{-x^{3}+5 x^{2}+2 x}{8(x-1)^{3}} .
\end{aligned}
$$

The $\bar{B} \rightarrow X_{s} \gamma$ branching ratio has been measured at several different experiments, including CLEO, BaBar, Belle, and ALEPH. The current experimental average from the Heavy Flavour Averaging Group is $[43,110]^{12}$

$$
\mathrm{BR}\left(\bar{B} \rightarrow X_{s} \gamma\right)_{\exp }=(3.55 \pm 0.24 \pm 0.09) \times 10^{-4}
$$

for a photon energy $E_{\gamma}>1.6 \mathrm{GeV}$.

$\operatorname{BR}\left(\bar{B} \rightarrow X_{s} \gamma\right)$ is known up to NNLO in QCD in the SM $[112,113]$. However, there is some tension between the experimental result and the two current SM predictions,

$$
\operatorname{BR}\left(\bar{B} \rightarrow X_{s} \gamma\right)_{\mathrm{SM}}=\left\{\begin{array}{l}
(3.15 \pm 0.23) \times 10^{-4} \text { (Misiak) } \\
(2.98 \pm 0.26) \times 10^{-4} \text { (Becher) }
\end{array}\right.
$$

which are respectively from Misiak et al [112] and Becher et al [113]. These SM predictions differ due to different approaches to handling higher-order contributions

\footnotetext{
${ }^{12}$ The most recent measurement from BaBar, which has not yet been incorporated into this average, reads $\operatorname{BR}\left(\bar{B} \rightarrow X_{s} \gamma\right)=(3.29 \pm 0.19 \pm 0.48) \times 10^{-4}[111]$
} 
to the photon energy cutoff corrections; however, this difference is within the $\pm 3 \%$ theoretical uncertainty due to uncalculated higher orders [112]. Relative to these SM predictions the experimental measurement yields

$$
R_{b \rightarrow s \gamma}^{\exp }=\left\{\begin{array}{l}
1.13 \pm 0.12 \text { (Misiak) } \\
1.19 \pm 0.13 \text { (Becher) }
\end{array}\right.
$$

Note that the SM predictions are roughly 1 to $1.5 \sigma$ below the experimental result. This respectively translates to $2 \sigma$ lower bounds of

$$
R_{b \rightarrow s \gamma}^{\mathrm{BSM}} \geq\left\{\begin{array}{l}
0.916 \text { (Misiak) } \\
0.952 \text { (Becher) },
\end{array}\right.
$$

on the prediction of the BSM model, where we have combined the experimental and BSM prediction uncertainties in quadrature.

It is expected that the strength of this constraint will soon be slightly improved due to new measurements from Belle II, a super $B$ factory experiment currently under construction at KEK in Japan. Conservative estimates expect that this experiment will reduce the experimental uncertainties on $\mathrm{BR}\left(\bar{B} \rightarrow X_{s} \gamma\right)$ to $7 \%$ with $5 \mathrm{ab}^{-1}$ of data, or $6 \%$ with $50 \mathrm{ab}^{-1}$ of data [114]. However, this is not a dramatic improvement over the current $7.2 \%$ experimental uncertainty. A more significant improvement in the strength of the $\operatorname{BR}\left(\bar{B} \rightarrow X_{s} \gamma\right)$ constraint would require a simultaneous reduction in the theoretical uncertainty.

\subsubsection{Dark matter candidates}

If a stable neutral scalar particle exists in an electroweak model, it becomes a weaklyinteracting massive particle (WIMP) candidate for dark matter. WIMPs are subject 
to constraints from dark matter direct-detection experiments and cosmological measurements.

In a standard thermal history of the universe, WIMPs would have been produced thermally in the early stages of the Big Bang. Initially, when the universe was very hot the density of dark matter would have been in thermal and chemical equilibrium with the SM particles; the SM particles would annihilate to create dark matter and vice versa. However, as the universe cooled the density of the dark matter particles would have become Boltzmann suppressed as the temperature dropped below the dark matter mass. As the temperature approached $m_{\mathrm{DM}} / 20,{ }^{13}$ the density of dark matter particles would have become so low that it became statistically unlikely for a dark matter particle to encounter its anti-particle and annihilate, causing the dark matter particles to 'freeze out' of thermal equilibrium.

Due to this process, the current observable relic abundance of dark matter in the universe is intimately related to the rate at which it can annihilate to SM particles. The observed relic abundance corresponds to a standard annihilation cross section of $\left\langle\sigma v_{r e l}\right\rangle_{\text {std }} \equiv 3 \times 10^{-26} \mathrm{~cm}^{3} / \mathrm{s}[115]$. The fraction of the dark matter density that is due to a neutral scalar $s^{0}, \Omega_{s^{0}} / \Omega_{\mathrm{DM}}$, can therefore be expressed as a ratio of the total scalar $s^{0} s^{0 *}$ annihilation cross section to the standard cross section,

$$
\frac{\Omega_{s^{0}}}{\Omega_{\mathrm{DM}}}=\frac{\left\langle\sigma v_{r e l}\right\rangle_{\mathrm{std}}}{\frac{1}{\eta}\left\langle\sigma v_{r e l}\left(s^{0} s^{0 *} \rightarrow \text { any }\right)\right\rangle},
$$

where $v_{r e l}$ is the relative velocity of the two colliding dark matter particles and the brackets indicate an average over the velocity distribution at the time of freeze-out. The symmetry factor $\eta$ in the denominator is $\eta=1$ for complex scalars and $\eta=2$ for real scalars; it accounts for differences in the probability that any given particle

\footnotetext{
${ }^{13}$ The freeze-out temperature required to achieve the observed relic abundance is roughly given by $m_{\mathrm{DM}} / 20$ regardless of the specific properties of the WIMP dark matter [43].
} 


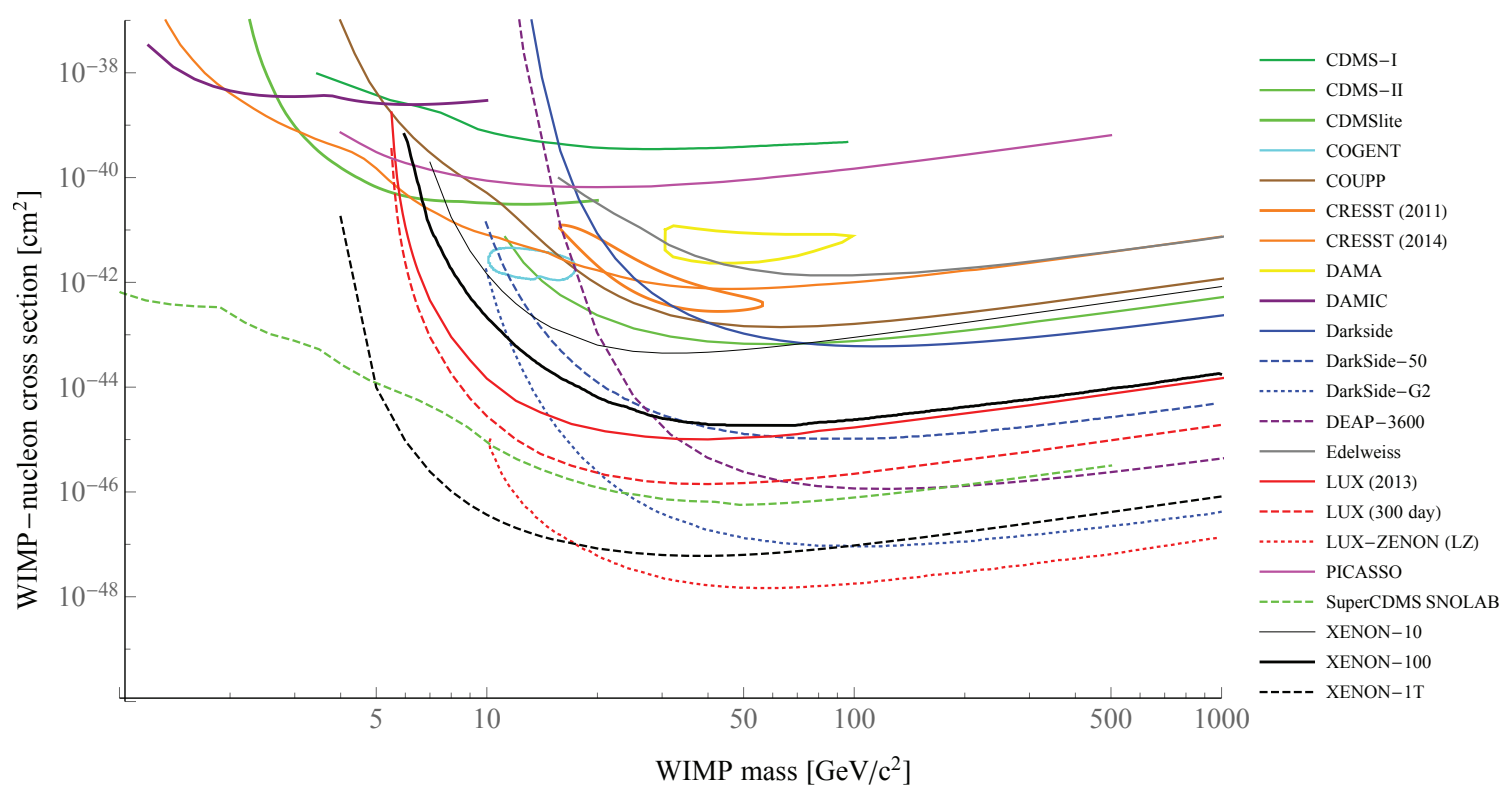

Figure 3.8: Summary of direct detection limits on the WIMP-nucleon interaction cross section, based on publicly available data from DMTools [116]. Current bounds are represented by solid lines, while dashed and dotted lines indicate projections for ongoing experiments.

meets its anti-particle and annihilates.

The density ratio of Eq. (3.109) has been strongly bounded by direct searches for dark matter at a variety of experiments. The majority of these direct-detection experiments search for evidence of the elastic scattering of dark matter particles from nuclei. In particular, measurements of the spin-independent, per-nucleon scattering cross section $\sigma_{\mathrm{SI}}^{s^{0}}$ for the dark matter candidate $s^{0}$ constrain the dark matter density fraction through the following relation,

$$
\frac{\Omega_{s^{0}}}{\Omega_{\mathrm{DM}}} \leq \frac{\sigma_{\mathrm{SI}}^{\text {limit }}}{\sigma_{\mathrm{SI}}^{s^{0}}},
$$

where $\sigma_{\mathrm{SI}}^{\text {limit }}$ is the experimental upper limit on the spin-independent dark matter scattering cross section.

Fig. 3.8 summarizes the current status (solid lines) and future projections 
(dashed lines) of dark matter direct-detection bounds from experiments searching for dark-matter-nucleon interactions. One will note that the bounds depend on the mass of the dark matter particle; candidates which are very light or very heavy are less constrained than those on the weak scale of $10 \mathrm{GeV}$ to $1 \mathrm{TeV}$. Two of the most competitive current bounds are provided by XENON-100 [117,118] and LUX [119,120], which currently require that the DM-nucleon scattering cross section be less than $10^{-9}$ to $10^{-8} \mathrm{pb}$ in the $10 \mathrm{GeV}$ to $1 \mathrm{TeV}$ mass region. However, these bounds are expected to improve by several orders of magnitude as these two experiments are upgraded (Xenon-1T [117], LZ [35]) and a series of next-generation experiments (e.g. DEAP3600 [121, 122], SuperCDMS [35], DarkSide [35]) begin to obtain results [123].

Dark matter candidates can also be bounded by direct searches at colliders. These bounds depend very strongly on the type (e.g. scalar, fermion, or axion) and specific properties of the dark matter under consideration, as the dark matter interactions will determine the production mechanism [62]. In particular, colliders generally provide the best limits at low masses below $10 \mathrm{GeV}$, where many other direct-detection experiments lack sensitivity, as seen in Fig. 3.8. However, as the dark matter candidates we consider are constrained by the $45 \mathrm{GeV}$ LEP limit discussed in Sec. 3.2.1, bounds in this low mass range are not relevant to our work.

We apply dark matter constraints to the $U(1)$ and $Z_{2}$-symmetric models of large scalar multiplets (see Secs. 5.3.4 and 6.3.5). These constraints will play a key role in excluding the majority of the $U(1)$ models. However, these bounds are not relevant to the Georgi-Machacek model considered in Chp. 7 as the neutral BSM scalars in this model are not stable. 


\section{Chapter 4}

\section{Constraints on the maximum}

\section{isospin and hypercharge of a scalar electroweak multiplet}

As discussed in Sec. 2.3.2, electroweak scalar multiplets have been implemented in BSM models for a large range of purposes, including mass generation and dark matter. The size $n$ (i.e. the number of isospin components in the multiplet) is intimately related to the total weak isospin $T$ through the relation $n=2 T+1$. Varying sizes of multiplets have been considered in the literature - from singlets $(n=1, T=0)$ to septets $(n=7, T=3)$ - and models with multiplets of $n \geq 3$ have become much more common in recent years. It is therefore interesting to consider whether an upper bound may be placed on the size (and by extension weak isospin) of a scalar electroweak multiplet.

In this chapter we consider constraints on the size $n$ and hypercharge $Y$ of an electroweak multiplet imposed from perturbative unitarity as described in Sec. 3.1.1. 


\subsection{A model with an extra scalar multiplet}

Consider an $S U(2)_{L}$ scalar multiplet of arbitrary size $n$. This could be a real multiplet $\Xi$ with zero hypercharge, or a complex multiplet $X$ of arbitrary hypercharge $Y$ :

$$
X=\left(\begin{array}{c}
\chi_{n} \\
\vdots \\
\chi_{2} \\
\chi_{1}
\end{array}\right), \quad \Xi=\left(\begin{array}{c}
\xi^{Q} \\
\vdots \\
\xi^{0} \\
\vdots \\
\xi^{-Q}
\end{array}\right)
$$

For $X$ we have $T^{3} \chi_{n}=T \chi_{n}, T^{3} \chi_{1}=-T \chi_{1}$, etc., where $T$ is the total isospin of the multiplet $X$ and $T^{3}$ is the third component of the isospin. In the case of the real multiplet $\Xi$ the hypercharge $Y=0$, the isospin $T$ must be integer (such that the electric charges $Q=T^{3}$ are also integer $),{ }^{1}$ and $\left(\xi^{Q}\right)^{*}=(-1)^{Q} \xi^{-Q}$.

The size and hypercharge of these multiplets can be limited by requiring that their tree-level scattering interactions with vector bosons preserve perturbative unitarity. As the size of the multiplet grows, more states will contribute to the relevant tree-level scattering processes of Figs. 3.1b-3.1d. Furthermore, the couplings involved in these interactions will also increase with size and hypercharge. The weak isospin $T$ associated with $S U(2)_{L}$ interactions will increase with $n=2 T+1$, and the $U(1)_{Y}$ couplings are proportional to $Y$. As a result, the tree-level amplitudes will grow with $n$ and $Y$ until they eventually violate the unitarity bound of Eq. (3.2).

Note that these constraints will apply to any scalar multiplet charged under $S U(2)_{L}$ or $U(1)_{Y}$. We make use only of the fact that such scalars must couple to the electroweak gauge bosons. Any mixing or vacuum expectation values associated with the scalar states of a model do not affect this bound, as we work entirely in the

\footnotetext{
${ }^{1}$ Real representations of $S U(2)$ must have integer $T$, as they are also representations of $S O(3)$.
} 
high energy limit where the effects of electroweak symmetry breaking are neglected. We also do not consider a particular scalar potential; including quartic interactions between scalars (as in Fig. 3.1a) would tighten the constraints, and so these bounds will be conservative.

By separating the interactions based on their symmetry group, we may obtain a bound on $Y$ in terms of $n$ from the $U(1)_{Y}$ amplitudes, and an independent bound on $n$ from the $S U(2)_{L}$ amplitudes. We will therefore work in the $\left[W_{\mu}^{ \pm}, W_{\mu}^{3}, B_{\mu}\right]$ gauge boson basis, where $B_{\mu}$ is still distinct from the $W^{ \pm, 3}$ bosons. The gauge interactions of the scalars will arise from the following scalar gauge-kinetic terms,

$$
\mathcal{L} \supset\left\{\begin{array}{l}
\left(\mathcal{D}_{\mu} X\right)^{\dagger}\left(\mathcal{D}^{\mu} X\right) \text { for } X \text { complex } \\
\frac{1}{2}\left(\mathcal{D}_{\mu} \Xi\right)^{\dagger}\left(\mathcal{D}^{\mu} \Xi\right) \text { for } \Xi \text { real. }
\end{array}\right.
$$

where in this basis the covariant derivative $\mathcal{D}_{\mu}$ is defined in Eq. (2.5). Using this Lagrangian, we derive the tree-level amplitudes for the scattering of a scalar anti-scalar pair into $B B, W^{3} B, W^{3} W^{3}$, and $W^{+} W^{-}$in the high energy limit. As described in Sec. 3.1.1, the amplitude is non-zero only if the gauge bosons have identical polarizations, in which case the total amplitudes are equivalent to the coupling coefficient in the quartic diagram (Eq. 3.11). Using Eq. (3.1), we find that the amplitudes involving a net-neutral pair of complex scalars $\chi_{k}^{*} \chi_{k}$ will contribute the following to the tree-level partial wave $a_{0}=\mathcal{M}_{0} / 16 \pi$ :

$$
\begin{aligned}
\mathcal{M}_{0}\left(\chi_{k}^{*} \chi_{k} \rightarrow B B / \sqrt{2}\right) & =g^{2} \frac{s_{W}^{2}}{c_{W}^{2}} \frac{Y^{2}}{2 \sqrt{2}}, \\
\mathcal{M}_{0}\left(\chi_{k}^{*} \chi_{k} \rightarrow B W^{3}\right) & =g^{2} \frac{s_{W}}{c_{W}} T^{3} Y, \\
\mathcal{M}_{0}\left(\chi_{k}^{*} \chi_{k} \rightarrow W^{3} W^{3} / \sqrt{2}\right) & =g^{2} \sqrt{2}\left(T^{3}\right)^{2}, \\
\mathcal{M}_{0}\left(\chi_{k}^{*} \chi_{k} \rightarrow W^{+} W^{-}\right) & =g^{2}\left[T(T+1)-\left(T^{3}\right)^{2}\right] .
\end{aligned}
$$


Here we have used the fact that initial or final states involving two identical particles receive an extra $1 / \sqrt{2}$ normalization.

For a pair of real scalars $\xi$ we find,

$$
\begin{aligned}
\mathcal{M}_{0}\left(\xi^{Q *} \xi^{Q} \rightarrow W^{3} W^{3} / \sqrt{2}\right) & =g^{2} \sqrt{2}\left(T^{3}\right)^{2} \quad \text { for } T^{3}=Q \geq 1, \\
\mathcal{M}_{0}\left(\xi^{Q *} \xi^{Q} \rightarrow W^{+} W^{-}\right) & =g^{2}\left[T(T+1)-\left(T^{3}\right)^{2}\right] \quad \text { for } T^{3}=Q \geq 1, \\
\mathcal{M}_{0}\left(\xi^{0} \xi^{0} / \sqrt{2} \rightarrow W^{+} W^{-}\right) & =g^{2} \frac{1}{\sqrt{2}} T(T+1) .
\end{aligned}
$$

The amplitude for $\xi^{0} \xi^{0} \rightarrow W^{3} W^{3}$ is zero, as are all amplitudes involving real scalars scattering to $B$ final states. Note that the main difference between the real and complex scalars is in the multiplicity of scalar states.

\subsection{General constraints from unitarity}

\subsection{1 $\mathrm{U}(1)_{Y}$ interactions}

To constrain the hypercharge $Y$ of the complex ${ }^{2}$ scalar multiplet $X$ in Eq. (4.1), we first consider the gauge interactions of scalars under $U(1)_{Y}$. From Eq. (4.3) we see that the zeroth partial wave amplitude for $\chi_{k}^{*} \chi_{k} \rightarrow B B / \sqrt{2}$ is the same for all $n$ members of the multiplet $X$. Including only $\mathrm{U}(1)_{Y}$ interactions, the coupled-channel amplitude matrix in the basis $\left(B B / \sqrt{2}, \chi_{1}^{*} \chi_{1}, \ldots, \chi_{n}^{*} \chi_{n}\right)$ is given by

$$
\mathcal{M}_{0}^{\mathrm{U}(1)}=\sqrt{2} g^{2} \frac{s_{W}^{2}}{c_{W}^{2}} \frac{Y^{2}}{2 \sqrt{2}}\left(\begin{array}{cccc}
0 & 1 & \cdots & 1 \\
1 & 0 & \cdots & 0 \\
\vdots & \vdots & & \vdots \\
1 & 0 & \cdots & 0
\end{array}\right),
$$

\footnotetext{
${ }^{2}$ Recall that by definition the real multiplet $\Xi$ has hypercharge of zero.
} 
where the $\sqrt{2}$ in front comes from the two contributing gauge boson polarization combinations. Due to its simplicity, this $n+1$-dimensional matrix can be diagonalized straightforwardly to obtain single eigenvalues of $\pm \sqrt{n}$ as well as $n-1$ zero eigenvalues. However, as shown in Sec. 3.1.1, we may also simplify the matrix by employing the linear combinations of states defined in Sec. 3.1.1. As the only possible combination of $U(1)$ gauge bosons is isospin-0, we make use of the linear combination of scalar states found in Eq. 3.15 to reduce Eq. (4.10) from an $n+$ 1-dimensional matrix to a $2 \times 2$ matrix. In the basis $\left(B B / \sqrt{2},\left[\chi^{*} \chi\right]_{0}\right) \mathcal{M}_{0}$ becomes

$$
\mathcal{M}_{0}^{\mathrm{U}(1)}=\frac{g^{2}}{16 \pi} \frac{s_{W}^{2}}{c_{W}^{2}} \frac{Y^{2}}{2} \sqrt{n}\left(\begin{array}{ll}
0 & 1 \\
1 & 0
\end{array}\right)
$$

The two non-zero eigenvalues of this matrix are equal in magnitude but different in sign. Using Eq. 3.3, the tree-level partial-wave amplitude $a_{0}$ corresponding to the positive (and largest) eigenvalue is given by

$$
a_{0}^{\max , \mathrm{U}(1)}=\frac{g^{2}}{16 \pi} \frac{s_{W}^{2}}{c_{W}^{2}} \frac{Y^{2}}{2} \sqrt{n} .
$$

Imposing the unitarity bound in Eq. (3.2), $\left|\operatorname{Re} a_{0}\right|<1 / 2$, and plugging in numbers, ${ }^{3}$ we obtain a constraint on the hypercharge as a function of the size of the multiplet,

$$
|Y| \lesssim \frac{19.8}{n^{1 / 4}}
$$

Note that when multiple $(N)$ hypercharged scalar multiplets $X_{j}(j=1, \ldots, N)$ are present, in a basis of $\left(B B / \sqrt{2},\left[X_{1}^{\dagger} X_{1}\right]_{0}, \ldots\left[X_{N}^{\dagger} X_{N}\right]_{0}\right)$, we would have the following

\footnotetext{
${ }^{3}$ We use $\alpha_{\mathrm{em}}=s_{W}^{2} g^{2} / 4 \pi \simeq 1 / 128$ and $s_{W}^{2} \simeq 0.231$. These values are valid at the weak scale; logarithmic renormalization-group running of $g$ and $g^{\prime}$ will cause numerical variations in our results at higher mass scales.
} 
partial wave matrix

$$
a_{0}=\left(\begin{array}{cccc}
0 & a_{0}^{\max , \mathrm{U}(1), 1} & \cdots & a_{0}^{\max , \mathrm{U}(1), N} \\
a_{0}^{\max , \mathrm{U}(1), 1} & 0 & \cdots & 0 \\
\vdots & \vdots & & \vdots \\
a_{0}^{\max , \mathrm{U}(1), N} & 0 & \cdots & 0
\end{array}\right) .
$$

As seen above, the largest eigenvalue of this matrix is just the sum in quadrature of the elements in the first row or first column; i.e. the sum in quadrature of the largest eigenvalue for each multiplet $X_{j}$. Therefore, the maximum bound would be attained by applying Eq. (3.2) to

$$
a_{0}^{\max , \mathrm{U}(1)}=\left[\sum_{j=1}^{N}\left(a_{0}^{\max , \mathrm{U}(1), j}\right)^{2}\right]^{1 / 2}
$$

\subsection{2 $\mathrm{SU}(2)_{L}$ interactions}

In this case we consider only the $S U(2)_{L}$ interactions of both the real and complex multiplets of Eq. (4.1) in order to bound their respective sizes $n$. We first consider the complex multiplet $X$. In this case, the coupled-channel amplitude matrix is more complicated. In the basis $\left(W^{+} W^{-}, W^{3} W^{3} / \sqrt{2}, \chi_{1}^{*} \chi_{1}, \ldots \chi_{n}^{*} \chi_{n}\right)$ it is given by

$$
\mathcal{M}_{0}^{\mathrm{SU}(2)}=\sqrt{2} \frac{g^{2}}{16 \pi}\left(\begin{array}{ccccc}
0 & 0 & A_{1} & \cdots & A_{n} \\
0 & 0 & B_{1} & \cdots & B_{n} \\
A_{1} & B_{1} & 0 & \cdots & 0 \\
\vdots & \vdots & \vdots & & \vdots \\
A_{n} & B_{n} & 0 & \cdots & 0
\end{array}\right),
$$

where again the $\sqrt{2}$ in front comes from the two contributing gauge boson polarization 
combinations, and

$$
\begin{aligned}
& A_{k}=T(T+1)-\left(T_{k}^{3}\right)^{2}, \\
& B_{k}=\sqrt{2}\left(T_{k}^{3}\right)^{2} .
\end{aligned}
$$

Here $T_{k}^{3}$ is the operator $T^{3}$ evaluated for the appropriate state $\chi_{k}^{*} \chi_{k}$ as defined in Eq. (3.13). Again, this matrix can be reduced to a simple block-diagonal by considering the linear combinations with isospin-0 and isospin-2 (as none of the pure $S U(2)_{L}$ vector boson combinations are isospin-1). Beginning with isospin-0, we use the $\left([W W]_{0},\left[\chi^{*} \chi\right]_{0}\right)$ basis defined by Eqs. (3.18) and (3.15). This yields the simplified coupled-channel amplitude sub-matrix

$$
\mathcal{M}_{0}^{0, \mathrm{SU}(2)}=g^{2} \frac{\left(n^{2}-1\right) \sqrt{n}}{2 \sqrt{3}}\left(\begin{array}{ll}
0 & 1 \\
1 & 0
\end{array}\right) .
$$

Recalling that $n=2 T+1$, the eigenvalues of this matrix correspond to the following tree-level partial wave amplitudes:

$$
a_{0}^{0, \mathrm{SU}(2)}= \pm \frac{g^{2}}{16 \pi} 2 T(T+1) \sqrt{\frac{n}{3}}= \pm \frac{g^{2}}{16 \pi} \frac{\left(n^{2}-1\right) \sqrt{n}}{2 \sqrt{3}} .
$$

Similarly, in the isospin-2 case we use the $\left([W W]_{2},\left[\chi^{*} \chi\right]_{2}\right)$ basis, with linear combinations defined by Eq. (3.19) and (3.17). We find

$$
\mathcal{M}_{0}^{\mathrm{SU}(2)}=g^{2} \frac{\sqrt{n\left(n^{2}-1\right)\left(n^{2}-4\right)}}{\sqrt{30}}\left(\begin{array}{ll}
0 & 1 \\
1 & 0
\end{array}\right),
$$

which yields the following tree-level partial wave amplitudes

$$
a_{0}^{2, \mathrm{SU}(2)}= \pm \frac{g^{2}}{16 \pi} \frac{\sqrt{n\left(n^{2}-1\right)\left(n^{2}-4\right)}}{\sqrt{30}},
$$

Here we have used

$$
\sum_{k}\left[T(T+1)-3\left(T_{k}^{3}\right)^{2}\right]^{2}=\frac{n\left(n^{2}-1\right)\left(n^{2}-4\right)}{20} .
$$


where $k$ sums over each element of the multiplet. Considering Eqs. (4.20) and (4.22), we can see that the largest eigenvalue of the zeroth partial wave amplitude matrix involving only $S U(2)_{L}$ interactions is that found from the isospin-0 combination,

$$
a_{0, \mathrm{complex}}^{\max \mathrm{SU}(2)}=\frac{g^{2}}{16 \pi} \frac{\left(n^{2}-1\right) \sqrt{n}}{2 \sqrt{3}} .
$$

A similar analysis for a real multiplet $\Xi$ yields

$$
a_{0, \text { real }}^{\max , \mathrm{SU}(2)}=\frac{1}{\sqrt{2}} a_{0, \mathrm{complex}}^{\max \mathrm{SU}(2)}=\frac{g^{2}}{16 \pi} \frac{\left(n^{2}-1\right) \sqrt{n}}{2 \sqrt{6}},
$$

where for a real multiplet $n$ must be an odd integer.

Imposing the unitarity bound in Eq. (3.2) upon the largest eigenvalue (Eqs. (4.24) and (4.25)) and plugging in numbers, we obtain an upper bound on the size (or isospin) of a single scalar multiplet from tree-level perturbative unitarity of the $\mathrm{SU}(2)_{L}$ interaction alone:

$$
\begin{array}{lll}
n \leq 8 & (T \leq 7 / 2) & \text { for a complex multiplet, } \\
n \leq 9 & (T \leq 4) & \text { for a real multiplet. }
\end{array}
$$

As in the $\mathrm{U}(1)_{Y}$ case, when $N$ scalar multiplets carrying isospin are present we have a coupled channel matrix of the same form as Eq. (4.14). Therefore the bound would be applied to the sum in quadrature of the largest eigenvalue (Eq. (4.24) or (4.25)) for each multiplet,

$$
a_{0}^{\max , \mathrm{SU}(2)}=\left[\sum_{j=1}^{N}\left(a_{0}^{\max , \mathrm{SU}(2), j}\right)^{2}\right]^{1 / 2} .
$$

\subsubsection{Combined electroweak gauge interactions}

Complex scalar multiplets that carry both isospin and hypercharge couple the $\mathrm{SU}(2)_{L}$ and $\mathrm{U}(1)_{Y}$ channels together, as well as introducing the additional isospin-1 $B W^{3}$ 
channel. In this case we would have a coupled channel matrix in the basis

$$
\left[B B / \sqrt{2}, B W^{3}, W^{3} W^{3} / \sqrt{2}, W^{ \pm} W^{ \pm}, \chi_{1}^{*} \chi_{1}, \ldots, \chi_{n}^{*} \chi_{n}\right]
$$

However, as before this matrix can be simplified by grouping the amplitudes into linear combinations by isospin according to Eqs. (3.15-3.19). In this case there are interactions of isospin 0,1 and 2, each of which corresponds to its own matrix as the combinations of different isospin are orthogonal.

In the case of isospin-0, we now have an amplitude matrix including both the $U(1)_{Y}$ and $S U(2)_{L}$ interactions seen above in the basis $\left(B B / \sqrt{2},[W W]_{0},\left[\chi^{*} \chi\right]_{0}\right)$. Evaluating this combined matrix is equivalent to considering the following matrix of maximum tree-level partial waves $a_{0}$,

$$
a_{0}^{\max , \mathrm{sym}}=\left(\begin{array}{ccc}
0 & 0 & a_{0}^{\max , \mathrm{U}(1)} \\
0 & 0 & a_{0}^{\max , \mathrm{SU}(2)} \\
a_{0}^{\max , \mathrm{U}(1)} & a_{0}^{\max , \mathrm{SU}(2)} & 0
\end{array}\right),
$$

where $a_{0}^{\max , \mathrm{U}(1)}$ and $a_{0}^{\max , \mathrm{SU}(2)}$ are respectively defined in Eqs. (4.12) and (4.24). As observed previously, the eigenvalues of this matrix are just the matrix elements summed in quadrature,

$$
a_{0}^{0, \mathrm{SU}(2) \times \mathrm{U}(1)}=\left[\left(a_{0}^{\max , \mathrm{U}(1)}\right)^{2}+\left(a_{0}^{\max , \mathrm{SU}(2)}\right)^{2}\right]^{1 / 2} .
$$

In the case of isospin-1, we have an amplitude matrix in the basis $\left(W^{3} B,\left[\chi^{*} \chi\right]_{1}\right)$, which gives

$$
\mathcal{M}_{0}^{\mathrm{SU}(2)}=g^{2} \frac{s_{W}}{c_{W}} \frac{Y \sqrt{n\left(n^{2}-1\right)}}{\sqrt{6}}\left(\begin{array}{ll}
0 & 1 \\
1 & 0
\end{array}\right) .
$$

The corresponding nonzero eigenvalues yield tree-level partial waves of

$$
a_{0}^{B W^{3}}=\frac{g^{2}}{16 \pi} \frac{s_{W}}{c_{W}} \frac{Y \sqrt{n\left(n^{2}-1\right)}}{\sqrt{6}},
$$




\begin{tabular}{ccccccccc}
\hline \hline$n$ & 1 & 2 & 3 & 4 & 5 & 6 & 7 & 8 \\
$T$ & 0 & $1 / 2$ & 1 & $3 / 2$ & 2 & $5 / 2$ & 3 & $7 / 2$ \\
$\left|Y_{\max }\right|$ & 19.8 & 16.7 & 15.1 & 14.0 & 13.0 & 12.1 & 10.8 & 8.3 \\
\hline \hline
\end{tabular}

Table 4.1: Upper limit on the hypercharge $Y$ allowed by perturbative unitarity for a complex $n$-plet of SU(2) $)_{L}$

Again, here we have included the extra factor of $\sqrt{2}$ coming from the two contributing gauge boson polarization combinations. For any values of $Y$ and $n$, this eigenvalue is always smaller than that of the isospin-0 case found in Eq. (4.30) above.

In the case of isospin-2, we have the exact same matrix as in Eq. (4.21), yielding the maximum eigenvalue of Eq. (4.22). This is also always smaller than the isospin-0 eigenvalue of Eq. (4.30) above.

As such, the largest eigenvalue is that given by the isospin-0 combination of $S U(2)_{L}$ and $U(1)_{Y}$ interactions in Eq. (4.30). By applying Eq. (3.2) to this partial wave amplitude one will obtain an upper bound on $Y$ from perturbative unitarity for a single multiplet of size $n$. Particular values for $Y$ and $n$ are tabulated in Table 4.1. Note in particular that in all cases a multiplet with $Y=2 T$ is allowed; in such a multiplet the state $\chi_{1}$ with $T^{3}=-T$ is electrically neutral. We will consider such a multiplet in Chp. 5. Note also that the septet with $T=3, Y=4-$ which can have a nonzero vacuum expectation value while preserving $\rho=1$ at tree level - is also allowed $[31,32]$.

We finally note that, when more than one scalar multiplet is present, the largest eigenvalue of the coupled-channel matrix can be found efficiently as follows. First, the maximum eigenvalues for the $\mathrm{U}(1)_{Y}$ and $\mathrm{SU}(2)_{L}$ interactions can be computed 
separately for each multiplet using Eqs. (4.12) and (4.24). Then the largest eigenvalue of the full coupled-channel system is just the largest eigenvalue of the following matrix,

$$
a_{0}^{\max , \mathrm{Sym}}=\left(\begin{array}{ccccc}
0 & 0 & a_{0}^{\max , \mathrm{U}(1), 1} & \cdots & a_{0}^{\max , \mathrm{U}(1), N} \\
0 & 0 & a_{0}^{\max , \mathrm{SU}(2), 1} & \cdots & a_{0}^{\max , \mathrm{SU}(2), N} \\
a_{0}^{\max , \mathrm{U}(1), 1} & a_{0}^{\max , \mathrm{SU}(2), 1} & 0 & \cdots & 0 \\
\vdots & \vdots & \vdots & & \vdots \\
a_{0}^{\max , \mathrm{U}(1), N} & a_{0}^{\max , \mathrm{SU}(2), N} & 0 & \cdots & 0
\end{array}\right),
$$

where we work in the basis $\left(B B / \sqrt{2},[W W]_{0},\left[X_{1}^{\dagger} X_{1}\right]_{0}, \ldots,\left[X_{N}^{\dagger} X_{N}\right]_{0}\right)$ and the index $1, \ldots, N$ counts the scalar multiplets.

In summary, an electroweak scalar multiplet may have a maximum size of $n \leq 8$ (9) if it is complex (real) (as in Eq. (4.26)), while the maximum hypercharge must satisfy $|Y| \lesssim 19.8 / n^{1 / 4}$ (as in Eq. (4.13)). If the scalar multiplet carries both isospin and hypercharge, its values are constrained by the values in Table 4.1. If more than one scalar multiplet is included in a model, the unitarity bounds upon the model can be computed using Eq. (4.33). 


\section{Chapter 5}

\section{Models with an accidental U(1)}

\section{symmetry}

As seen in the previous chapter, electroweak scalar multiplets as large as $n=8$ (with weak isospin $T=7 / 2$ ) are permitted by tree-level perturbative unitarity if the scalars are complex ( $n=9, T=4$ if real). Many models with multiplets of size $1 \leq n \leq 4$ have been considered at length in the literature. However, studies of models that extend the SM by a large scalar multiplet of size $n \geq 5$ are still relatively rare. Currently the primary examples are quintet $(n=5, T=2)$ models for neutrino mass generation [25-27], a septet Higgs model $(n=7, T=3)$ [31-33], and dark matter models with $n=5,7,9$ [14-16]. It is therefore interesting to consider the constraints that may be placed on a broader class of $5 \leq n \leq 8$ scalar multiplets.

Of particular interest are models which preserve a global $U(1)$ or $Z_{2}$ symmetry in the scalar potential, as such models will include a dark matter candidate. If all interaction terms are invariant under $U(1)\left(S \rightarrow S e^{i \theta}\right)$ or $Z_{2}(S \rightarrow-S)$ transformations in the scalar multiplet $S$, the individual scalar states $s_{i}$ will only be permitted 
to decay to final states including other $s_{j}$, which will not be kinematically available to the lightest scalar. As a result, the lightest member of the multiplet will be stable, and thus a dark matter candidate. Given that electrically charged relics are either excluded or severely constrained by experimental evidence, this dark matter candidate is required to be electrically neutral.

These additional symmetries can arise accidentally at the renormalizable level, or be imposed by hand. They are particularly common in models which extend the SM by a $n \geq 5$ scalar multiplet, as the preservation of the electroweak symmetry in Lagrangian terms coupling the SM doublet $\Phi$ and the large multiplet $X$ naturally leads to a structure which favours an additional $U(1)$ or $Z_{2}$. Furthermore, these additional symmetries are not permitted to be broken spontaneously through a vacuum expectation value as in the Higgs Mechanism. The spontaneous breaking of an additional global $U(1)$ symmetry would lead to an additional massless Goldstone boson (as discussed in Sec. 2.2.3) which would mediate a long-range fermion interaction that has not been experimentally observed. Similarly, the spontaneous breaking of an additional $Z_{2}$ symmetry through a vacuum expectation value of a large multiplet is tightly constrained by the $\rho$ parameter (see Sec. 3.2.2). As such, we will not permit our large scalar multiplets to have a vacuum expectation value.

In this chapter we will consider the phenomenology of models which preserve an additional global $U(1)$ symmetry. This $U(1)$ will arise accidentally in models with an additional complex multiplet of size $5 \leq n \leq 8$ and hypercharge $Y=2 T$. This choice of hypercharge also allows the lightest stable scalar to be neutral. In Sec. 5.2 we apply constraints from perturbative unitarity, while in Sec. 5.3 we consider experimental bounds from oblique parameters, $h \rightarrow \gamma \gamma$, direct collider searches, and dark matter direct-detection. The $Z_{2}$ cases will be similarly studied in Chapter 6 . 


\subsection{Model description}

We consider a class of models that extend the SM through the addition of a single complex scalar multiplet $X$, with hypercharge $Y=2 T$ (normalized so that $Q=T^{3}+$ $Y / 2$ ). As seen in Chp. 4, electroweak scalar multiplets may have a size $1 \leq n \leq 8$, and the $Y=2 T$ case is allowed by perturbative unitarity for all of these cases. The choice of hypercharge allows the lightest member of $X$ to be neutral. Furthermore, when $n \geq 5$ the scalar potential possesses an accidental global U(1) symmetry corresponding to phase rotations of $X$. This $\mathrm{U}(1)$ symmetry ensures that the lightest member of $X$ is stable - at least at the level of renormalizable operators - allowing for a dark matter candidate in the model.

We will study the $5 \leq n \leq 8$ cases where the lightest member is both neutral and stable. The scalar content of the model consists of the Higgs doublet $\Phi(T=1 / 2$, $Y=0)$ and the single large complex scalar multiplet $X(T=(n-1) / 2, Y=2 T)$,

$$
\Phi=\left(\begin{array}{c}
\phi^{+} \\
\phi^{0}
\end{array}\right)=\frac{1}{\sqrt{2}}\left(\begin{array}{c}
0 \\
h+v
\end{array}\right), \quad X=\left(\begin{array}{c}
\chi^{+(n-1)} \\
\vdots \\
\chi^{+} \\
\chi^{0}
\end{array}\right)
$$

where the superscript on $\chi^{Q}$ indicates the electric charge of the state $Q=T^{3}+Y / 2$, and $v \simeq 246 \mathrm{GeV}$ is the SM Higgs vacuum expectation value (vev). We assume that the BSM multiplet $X$ does not acquire a vev. In Eq. (5.1), we write the Higgs doublet in both the Goldstone basis $\phi^{ \pm, 0}$ and the rotated (unitarity gauge) Higgs $h$ basis after electroweak symmetry breaking, as we will need the Goldstone basis when applying unitarity constraints in the high-energy limit. 
The gauge-invariant scalar potential is given by

$$
\begin{aligned}
V(\Phi, X)= & m^{2} \Phi^{\dagger} \Phi+M^{2} X^{\dagger} X+\lambda_{1}\left(\Phi^{\dagger} \Phi\right)^{2}+\lambda_{2} \Phi^{\dagger} \Phi X^{\dagger} X \\
& +\lambda_{3} \Phi^{\dagger} \tau^{a} \Phi X^{\dagger} T^{a} X+\mathcal{O}\left(X^{4}\right)
\end{aligned}
$$

where $\tau^{a}$ and $T^{a}$ are the generators of $\mathrm{SU}(2)_{L}$ in the doublet and $n$-plet representations, respectively. We do not explicitly write down the quartic scalar $X$ interaction terms here, as they are not necessary for the work below. The interactions of the scalars with gauge bosons are defined analogously to Eq. (4.2); the specifics of the Feynman rules can be found in Appendix A.1.

The mass of particle $\chi^{Q}$ with charge $Q=T^{3}+Y / 2 \geq 0$ is given by

$$
m_{\chi^{Q}}^{2}=M^{2}+\frac{1}{2} v^{2}\left[\lambda_{2}-\frac{1}{2} \lambda_{3}\left(Q-\frac{n-1}{2}\right)\right] \equiv M^{2}+\frac{1}{2} v^{2} \Lambda_{Q}
$$

where we define the dimensionless couplings $\Lambda_{Q}$ as the quantity in square brackets above. The neutral particle $\chi^{0}$, with $T^{3}=-T=-(n-1) / 2$, will have a mass,

$$
m_{\chi^{0}}^{2}=M^{2}+\frac{1}{2} v^{2}\left[\lambda_{2}+\frac{1}{4} \lambda_{3}(n-1)\right]=M^{2}+\frac{1}{2} v^{2} \Lambda_{0}
$$

The masses of the charged states $\chi^{Q}$ can be written in terms of the $\chi^{0}$ mass as

$$
m_{\chi^{Q}}^{2}=m_{\chi^{0}}^{2}-\frac{1}{4} v^{2} \lambda_{3} Q
$$

In order for the lightest member of $X$ to be electrically neutral, we require that $\lambda_{3}<0$.

\subsection{Theoretical constraints}

For this class of models we will consider only theoretical constraints on the scalar potential parameters from tree-level perturbative unitarity. As we will show in the 
next section, for $n=6,7,8$ this class of models can be excluded by unitarity and experimental constraints without considering quartic interactions involving the BSM scalars $\chi^{Q}$. In the $n=5$ case, a dimension-5 Planck-suppressed interaction will affect the stability of the light neutral state, requiring further analysis of the scalar potential in order to determine its viability. As a result, we do not consider constraints on these models from the boundedness of the potential or alternative vacua.

\subsubsection{Constraints from tree-level perturbative unitarity}

In the scalar potential Eq. (5.2), interactions involving $X$ are controlled by two free parameters, $\lambda_{2}$ and $\lambda_{3}$. Both of these parameters can be bounded by requiring perturbative unitarity of the zeroth partial wave amplitude. The parameter $\lambda_{2}$ controls the strength of the isospin-zero $\chi^{*} \chi \rightarrow \phi^{*} \phi$ amplitude, while $\lambda_{3}$ controls the strength of the isospin-one $\chi^{*} \chi \rightarrow \phi^{*} \phi$ channel. As a result, the procedure of decomposing the matrix of coupled amplitudes by isospin is particularly helpful (see Sec. 3.1.1), as it allows us to obtain separate bounds for each parameter.

In this procedure, we consider the amplitudes for scalar-to-scalar scattering according to Eq. (3.4) and scalar-to-vector scattering according to Eq. (3.11). We work in the high-energy limit where the Higgs doublet is defined in the Goldstone basis $\phi^{+}, \phi^{0}$ of Eq. (5.1), and only the transverse polarizations are considered for gauge bosons. The scattering processes may therefore involve SM scalars $\phi^{+}, \phi^{0}$ or BSM scalars $\chi^{Q}$, as well as their antiparticles.

However, in constructing this matrix we consider only scalar-vector interactions involving $\chi^{Q}$, and scalar-to-scalar interactions that couple doublet scalars $\phi^{+}, \phi^{0}$ to multiplet scalars $\chi^{Q}$. We neglect all interactions of the form $\phi^{\dagger} \phi \rightarrow V V, \phi^{\dagger} \phi$; these 
contributions are much smaller than those involving multiplet states $\chi^{Q}$ as $\phi^{\dagger} \phi \rightarrow V V$ are proportional to $g^{2}(g \approx 0.67), \phi^{\dagger} \phi \rightarrow \phi^{\dagger} \phi \propto \lambda_{1} \simeq 1 / 8$, and neither are enhanced by the large multiplet size $n$. Furthermore, neglecting amplitudes that are quartic in $\phi$ and $\chi^{Q}$ allows us to bound $\lambda_{2,3}$ without any dependence on $\lambda_{1}$ or the $X$ quartic couplings. We have numerically verified that the inclusion of these interactions will always tighten the bounds on $\lambda_{2}$ and $\lambda_{3}$, although the constraint will depend on the interplay between the $\lambda_{1}$ contributions and those from the quartic $X$ couplings. We therefore obtain conservative bounds on $\lambda_{2}$ and $\lambda_{3}$ by neglecting these processes.

We consider first the isospin-0 amplitudes, which will bound $\lambda_{2}$. We define isospin-0 linear combinations of the BSM scalar states $\chi^{Q}$ according to Eq. (3.15), while the gauge boson combinations are given by Eqs. (3.18) and $B B / \sqrt{2}$. The appropriate combination of doublet states $\phi$ is define by Eq. (3.15) in the case that $n=2$, which gives

$$
\left[\phi^{*} \phi\right]_{0}=\frac{1}{\sqrt{2}}\left(\phi^{+} \phi^{-}+\phi^{0 *} \phi^{0}\right) .
$$

Using the Feynman rules found in Appendix A.1, the relevant amplitudes $\mathcal{M}_{0}$ are found to be

$$
\begin{aligned}
\mathcal{M}_{0}\left(\left[\chi^{*} \chi\right]_{0} \rightarrow\left[\phi^{*} \phi\right]_{0}\right) & =-2 \sqrt{n} \lambda_{2}, \\
\mathcal{M}_{0}\left(\left[\chi^{*} \chi\right]_{0} \rightarrow[W W]_{0}\right) & =g^{2} \frac{\left(n^{2}-1\right) \sqrt{n}}{2 \sqrt{3}}, \\
\mathcal{M}_{0}\left(\left[\chi^{*} \chi\right]_{0} \rightarrow B B / \sqrt{2}\right) & =g^{2} \frac{s_{W}^{2}}{c_{W}^{2}} \frac{Y^{2} \sqrt{n}}{2}=g^{2} \frac{s_{W}^{2}}{c_{W}^{2}} \frac{(n-1)^{2} \sqrt{n}}{2},
\end{aligned}
$$

where the $\chi^{*} \chi \rightarrow W W, B B$ amplitudes have been multiplied by $\sqrt{2}$ to include both of the contributing transverse gauge boson polarization combinations and we used $Y=2 T=n-1$ in the last line.

Therefore, in the basis $\left(\left[\chi^{*} \chi\right]_{0},[W W]_{0}, \frac{B B}{\sqrt{2}},\left[\phi^{*} \phi\right]_{0}\right)$, the isospin-0 coupled chan- 
nel amplitude matrix is

$$
\mathcal{M}_{0}^{(0)}=\left(\begin{array}{cccc}
0 & \frac{g^{2}}{2 \sqrt{3}}\left(n^{2}-1\right) \sqrt{n} \frac{g^{2} s_{W}^{2}}{2 c_{W}^{2}} Y^{2} \sqrt{n}-\sqrt{2 n} \lambda_{2} \\
\frac{g^{2}}{2 \sqrt{3}}\left(n^{2}-1\right) \sqrt{n} & 0 & 0 & 0 \\
\frac{g^{2} s_{W}^{2}}{2 c_{W}^{2}} Y^{2} \sqrt{n} & 0 & 0 & 0 \\
-\sqrt{2 n} \lambda_{2} & 0 & 0 & 0
\end{array}\right) .
$$

As seen in the preceding chapter, the eigenvalues of a matrix of this form are given by the sum in quadrature of the elements of the first row or first column. Using this and Eq. (3.3), we find that the zeroth partial wave amplitude $a_{0}$ given by the largest eigenvalue is

$$
a_{0}^{\max , 0}=\frac{\sqrt{n}}{16 \pi} \sqrt{\frac{g^{4}}{12}\left(n^{2}-1\right)^{2}+\frac{g^{4} s_{W}^{4}}{4 c_{W}^{4}} Y^{4}+2 \lambda_{2}^{2}},
$$

Applying Eq. (3.2) to this eigenvalue and using $Y=n-1$, we obtain the following limit on $\lambda_{2}$,

$$
\begin{aligned}
\left|\lambda_{2}\right| & \leq \sqrt{\frac{32 \pi^{2}}{n}-g^{4} \frac{\left(n^{2}-1\right)^{2}}{24}-g^{4} \frac{s_{W}^{4}}{c_{W}^{4}} \frac{Y^{4}}{8}}, \\
& =\sqrt{\frac{32 \pi^{2}}{n}-g^{4} \frac{\left(n^{2}-1\right)^{2}}{24}-g^{4} \frac{s_{W}^{4}}{c_{W}^{4}} \frac{(n-1)^{4}}{8}} .
\end{aligned}
$$

The isospin-1 case yields a similar bound on $\lambda_{3}$. We work in the basis of $\left(\left[\chi^{*} \chi\right]_{1}, W^{3} B,\left[\phi^{*} \phi\right]_{1}\right)$, where the normalized isospin-1 field combination of $\chi$ states is defined by Eq. (3.16) and the $\phi$ combination is analogously defined for $n=2$ as

$$
\left[\phi^{*} \phi\right]_{1}=\frac{1}{\sqrt{2}}\left(\phi^{+} \phi^{-}-\phi^{0 *} \phi^{0}\right) .
$$

The relevant amplitudes for the isospin-one channels are

$$
\begin{aligned}
\mathcal{M}_{0}\left(\left[\chi^{*} \chi\right]_{1} \rightarrow\left[\phi^{*} \phi\right]_{1}\right) & =-\frac{\sqrt{n\left(n^{2}-1\right)}}{2 \sqrt{6}} \lambda_{3}, \\
\mathcal{M}_{0}\left(\left[\chi^{*} \chi\right]_{1} \rightarrow\left[W^{3} B\right]_{1}\right) & =g^{2} \frac{s_{W}}{c_{W}} \frac{Y \sqrt{n\left(n^{2}-1\right)}}{\sqrt{6}}=g^{2} \frac{s_{W}}{c_{W}} \frac{(n-1) \sqrt{n\left(n^{2}-1\right)}}{\sqrt{6}},
\end{aligned}
$$




\begin{tabular}{|c|cccc|}
\hline$n$ & 5 & 6 & 7 & 8 \\
\hline$\left|\lambda_{2}^{\lim }\right|$ & 7.64 & 6.49 & 5.01 & 2.17 \\
\hline$\left|\lambda_{3}^{\lim }\right|$ & 11.1 & 8.17 & 6.11 & 4.41 \\
\hline
\end{tabular}

Table 5.1: Upper limits on $\left|\lambda_{2}\right|$ and $\left|\lambda_{3}\right|$ from unitarity, for $Y=2 T=n-1$.

where again the $\chi^{*} \chi \rightarrow W^{3} B$ amplitude includes both of the contributing transverse gauge boson polarization combinations. The coupled-channel amplitude matrix is then given by

$$
\mathcal{M}_{0}^{(1)}=\frac{1}{32 \sqrt{6} \pi} \sqrt{n\left(n^{2}-1\right)}\left(\begin{array}{ccc}
0 & \frac{2 g^{2} s_{W}}{c_{W}} Y & -\lambda_{3} \\
\frac{2 g^{2} s_{W}}{c_{W}} Y & 0 & 0 \\
-\lambda_{3} & 0 & 0
\end{array}\right) .
$$

Here the largest eigenvalue yields the following partial wave amplitude,

$$
a_{0}^{\max , 1}=\frac{1}{32 \sqrt{6} \pi} \sqrt{n\left(n^{2}-1\right)} \sqrt{\lambda_{3}^{2}+\frac{4 g^{4} s_{W}^{2}}{c_{W}^{2}} Y^{2}},
$$

which leads to the bound

$$
\begin{aligned}
\left|\lambda_{3}\right| & \leq 2 \sqrt{\frac{384 \pi^{2}}{n\left(n^{2}-1\right)}-g^{4} \frac{s_{W}^{2}}{c_{W}^{2}} Y^{2}}, \\
& =2 \sqrt{\frac{384 \pi^{2}}{n\left(n^{2}-1\right)}-g^{4} \frac{s_{W}^{2}}{c_{W}^{2}}(n-1)^{2}} .
\end{aligned}
$$

Recall that $\lambda_{3}$ must be negative so that $\chi^{0}$ is the lightest member of the large multiplet, but $\lambda_{2}$ can have either sign. Numerical bounds for both $\lambda_{2}$ and $\lambda_{3}$ are given for $n=5,6,7$, and 8 in Table $5.1 .^{1}$

\footnotetext{
${ }^{1}$ In this table we use $g^{2}=4 \pi \alpha / s_{W}^{2}, s_{W}^{2}=0.231$, and $\alpha=1 / 128$.
} 


\subsection{Experimental constraints}

\subsubsection{Constraints from oblique parameters}

The multiplet $X$ contributes to electroweak observables through the oblique parameters $S, T$, and $U$ [84]. Substituting Eq. (3.38) into the definition of $S$ given in Eq. (3.31) for each scalar state $\chi^{Q}$, one can show that the contribution to the $S$ parameter from a complex scalar multiplet obeying a U(1) global symmetry is given by

$$
S=-\frac{Y}{6 \pi} \sum_{Q=0}^{n-1} T_{Q}^{3} \log \left(m_{\chi^{Q}}^{2}\right) .
$$

Similarly, using Eq. (3.36) and Eq. (3.49) in the definition Eq. (3.32), the contribution from $X$ to the $T$ parameter can be found to be

$$
\begin{aligned}
T=\frac{1}{4 \pi m_{Z}^{2} s_{W}^{2} c_{W}^{2}}\left[\sum_{Q=0}^{n-1} m_{\chi^{Q}}^{2} \log \left(m_{\chi^{Q}}^{2}\right)\left[T(T+1)-\left(T_{Q}^{3}\right)^{2}\right]\right. \\
\left.\quad-\sum_{Q=0}^{n-2}\left(T-T_{Q}^{3}\right)\left(T+T_{Q}^{3}+1\right) f_{2}\left(m_{Q}, m_{Q+1}\right)\right] .
\end{aligned}
$$

Finally, using Eq. (3.38) in Eq. (3.33) for each $\chi^{Q}$ results in the contribution to $U$ from $X$,

$$
U=\frac{1}{\pi}\left[\sum_{Q=0}^{n-2}\left(T-T_{Q}^{3}\right)\left(T+T_{Q}^{3}+1\right) f_{1}\left(m_{Q}, m_{Q+1}\right)-\frac{1}{3} \sum_{Q=0}^{n-1}\left(T_{Q}^{3}\right)^{2} \log \left(m_{Q}^{2}\right)\right] .
$$

We have recalculated and verified these formulas, which were originally computed for arbitrary isospin and hypercharge in Ref. [88]. Here the index sums over the electric charge $Q$ of the scalars $\chi^{Q}, T=\frac{n-1}{2}$ is the total weak isospin of the multiplet, $T_{Q}^{3}=Q-T$ is its third component for the scalar $\chi^{Q}, m_{\chi^{Q}}$ is the mass defined by Eq. (5.5), and the functions $f_{1}$ and $f_{2}$ are defined by Eqs. (3.39) and (3.37).

The contributions to the oblique parameters depend on $n, Y$, and the masses $m_{\chi^{Q}}$ of each state in the multiplet. For a given $n$ and with $Y=2 T=n-1$, the 

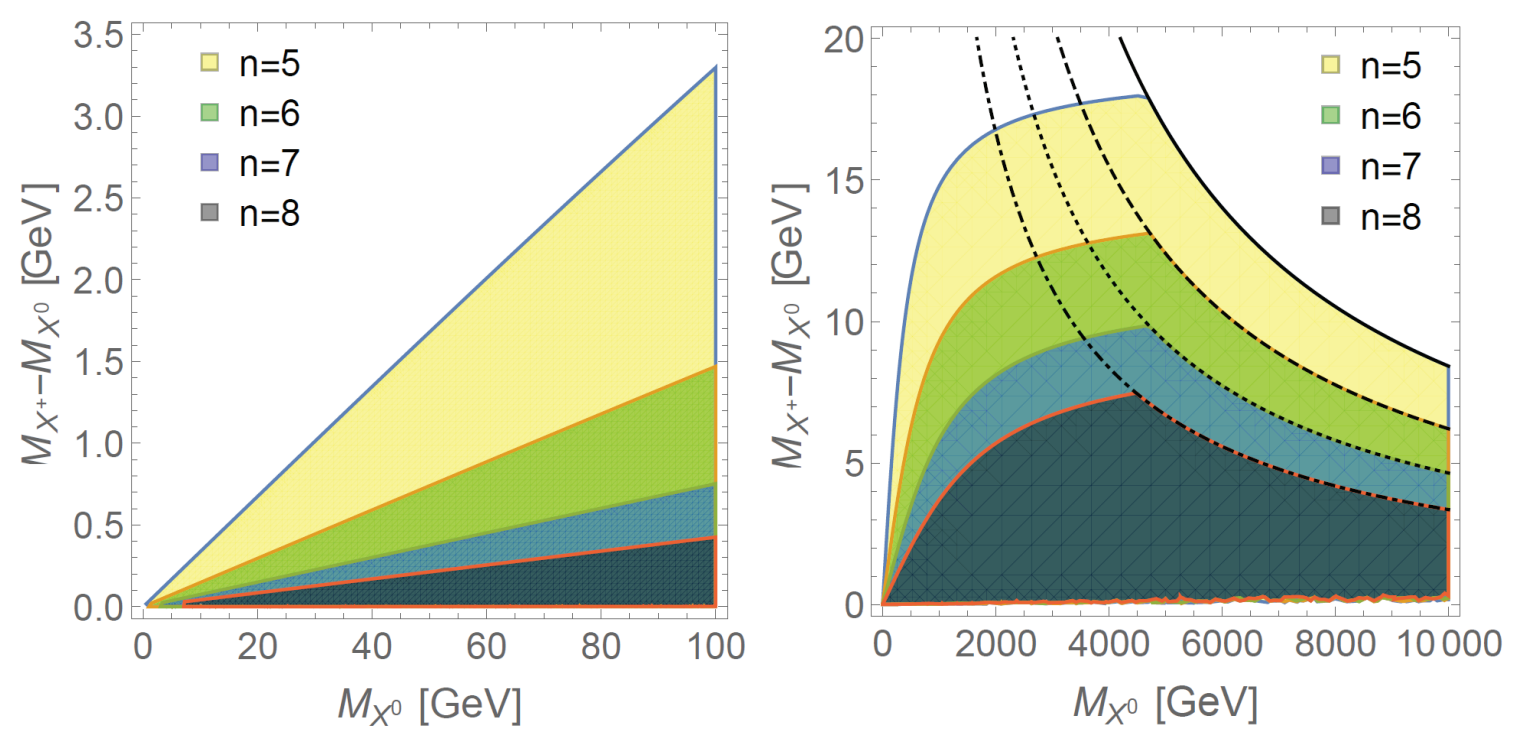

Figure 5.1: The $95 \%$ confidence level constraints on $\Delta m \equiv m_{\chi^{+1}}-m_{\chi^{0}}$ as a function of $m_{\chi^{0}}$ from the $S, T$, and $U$ parameters for the scalar multiplets with $n=5,6,7$, and 8 and $Y=2 T=n-1$. Dashed lines indicate the upper limit on $\Delta m$ from the unitarity bound on $\lambda_{3}$. The left panel shows the low- $m_{\chi^{0}}$ region while the right panel extends to higher masses.

oblique parameters constrain only the scalar masses, which depend on three model parameters from the scalar potential: $M, \lambda_{2}$, and $\lambda_{3} . M$ and $\lambda_{2}$ always arise together in a fixed combination that sets the overall mass scale, while $\lambda_{3}$ controls the mass splitting. As a result, we may use the experimental bounds on the oblique parameters to constrain the lightest mass $m_{\chi^{0}}$ and the first mass splitting $\Delta m \equiv m_{\chi^{+}}-m_{\chi^{0}}$.

The current experimental values for $S, T$ and $U$ are given by Eq. (3.52). We use these values to constrain $m_{\chi^{0}}$ and $\Delta m$ using the three-observable $\chi^{2}$ variable defined in Eq. (3.50). As we are bounding two parameters, we use a $\chi^{2}$ fit with two degrees of freedom, $\chi_{0.05}^{2}=5.99$.

The limits due to this constraint from oblique parameters are shown in Fig. 5.1 for models $5 \leq n \leq 8$. At low $m_{\chi^{0}}$ the constraint is dominated by the $S$ parameter and leads to an upper bound on $\Delta m$ that is linear in $m_{\chi^{0}}$. This bound can be 
parametrized as

$$
\Delta m \equiv m_{\chi^{+}}-m_{\chi^{0}}=0.039\left[\frac{1}{n-4}-0.20\right] m_{\chi^{0}}
$$

For larger $m_{\chi^{0}} \sim 1 \mathrm{TeV}$, the constraint from the $T$ parameter becomes important and limits the value of $\Delta m$ independent of $m_{\chi^{0}}$. For $m_{\chi^{0}} \sim 5-6 \mathrm{TeV}$, the unitarity limit on $\lambda_{3}$ becomes the strongest constraint on $\Delta m$, as shown by the dashed lines in Fig. 5.1. Notice that for $n \geq 6$ the mass splitting between $\chi^{0}$ and the next-lightest state $\chi^{+}$is constrained to be no more than about $12 \mathrm{GeV}$; for $m_{\chi^{0}} \sim 100 \mathrm{GeV}$ the splitting is less than $1.3 \mathrm{GeV}$. The maximum allowed mass splitting decreases with increasing $n$.

One might notice a small upward shift in the allowed parameter space of Fig. 5.1 when it is compared with the same plots in the original publication, Ref. [2]. There is also a corresponding change in the parametrization of Eq. (5.24) $(\{0.031,0.13\}$ compared with $\{0.039,0.20\})$. These differences are due to an update in the experimental constraints on the oblique parameters [89] since the original publication of this work in 2012 (which made use of Ref. [124]). The update includes a new QCD correction to $m_{W}$ and improved NNLO calculations of the $Z$ boson partial widths and branching ratios in the global fit used to extract the experimental constraints on $S, T$ and $U$. However, the overall effect of the update is small and does not alter our conclusions (apart from Eq. (5.24)).

\subsubsection{Constraints from $h \rightarrow \gamma \gamma$ decay}

Charged scalars $\chi^{Q}$ with $Q \neq 0$ are also bounded by $h \rightarrow \gamma \gamma$ decay. The decay width is defined by Eq. (3.57), where contributions from the $W$ boson and the top quark are respectively given by Eq. (3.60) and (3.65) with $\kappa_{f}=\kappa_{W}=1$. We neglect 
contributions from light quarks. For the $U(1)$ models, the contribution from charged scalar particles becomes

$$
A_{S}^{h \gamma \gamma}=v^{2} \sum_{Q} \frac{2 \Lambda_{0}-\lambda_{3} Q}{m_{h}^{2}} Q^{2}\left[1-\frac{4 m_{\chi^{0}}^{2}-v^{2} \lambda_{3} Q}{m_{h}^{2}} f\left(\frac{4 m_{\chi^{0}}^{2}-v^{2} \lambda_{3} Q}{m_{h}^{2}}\right)\right],
$$

where we have defined the Higgs couplings in terms of $\Lambda_{0}$ using Eqs. (5.3) and (5.4), and we have defined the scalar masses using Eq. (5.5). The function $f(\tau)$ is defined by Eq. (3.63).

As shown by Eq. (5.25), the experimental LHC bounds on $h \rightarrow \gamma \gamma$ in Table 3.1 may be used to constrain a combination of $m_{\chi^{0}}, \lambda_{3}$ and $\Lambda_{0}$. A bound on $\Lambda_{0}$ may equivalently be expressed as a bound on $\lambda_{2}$ for a given $\lambda_{3}$, as defined in Eq. (5.4). For a particular neutral scalar mass $m_{\chi^{0}}$ one can use the constraint from oblique parameters - easily approximated by Eq. (5.24) for $m_{\chi^{0}} \leq 1 \mathrm{TeV}$ - to limit $\lambda_{3}$, and then bound $\Lambda_{0}$ (or $\lambda_{2}$ ) using the extrema of the $\lambda_{3}$ range. We implement this constraint numerically in Sec. 5.3.4.

\subsubsection{Constraints from direct searches}

The BSM scalars $\chi^{Q}$ are subject to the direct-search constraints from $Z$ and Higgs decay discussed in Sec. 3.2.1. As we have chosen $\chi^{0}$ to be the lightest scalar of the multiplet, the bound from $Z$ decay will be satisfied so long as

$$
m_{\chi^{0}} \gtrsim m_{Z} / 2 \simeq 45 \mathrm{GeV}
$$

As we will show in the following sections, this lower bound from $Z$ decay along with oblique, $h \rightarrow \gamma \gamma$, and dark matter constraints will be sufficient to entirely exclude the $n=6,7,8$ models. We will therefore only briefly comment on the possibilities for constraints from Higgs decay here. As shown in Ref. [2], the model parameters may 
also be constrained using the Higgs decay widths $\Gamma\left(h \rightarrow \chi^{Q *} \chi^{Q}\right)$, computed according to Eq. (3.20). These widths may be used to compute the branching ratios, which may in turn be bounded by ATLAS limits on $\mathrm{BR}(h \rightarrow$ invisible $)$ or by using measured Higgs signal strengths from the LHC in conjunction with Eq. (3.23). Under the assumption that oblique constraints are used to bound $\lambda_{3}$, these Higgs decay bounds can be used to further constrain $\lambda_{2}$; for example, $\left|\lambda_{2}\right| \lesssim 0.015$ for $m_{\chi^{0}} \leq 55 \mathrm{GeV}[2]$ using the experimental results of Refs. $[73,74]$.

\subsubsection{Dark matter constraints}

In the $n=6,7,8$ models, the lightest neutral scalar is stable, and therefore becomes a candidate for dark matter. For these cases, the allowable relic density of $\chi^{0(*)}$ is constrained by its non-observation in direct dark matter detection experiments. In

particular, the fraction of the ambient dark matter density (Eq. (3.109)) that can be attributed to $\chi^{0(*)}$ is bounded from above according to Eq. (3.110).

However, this limit will not apply to the model with $n=5$. In this model the isospin of the scalar multiplet allows a dimension-5 Planck-suppressed interaction with the SM Higgs doublet in the scalar potential. This term will have form

$$
\mathcal{L} \supset \frac{1}{M_{P l}} \Phi \Phi \Phi \Phi X^{\dagger}+\text { h.c. }
$$

where $M_{P l}$ is the Planck mass. This operator induces a mixing of the SM Higgs into the neutral component of $X, \chi^{\prime}=\chi^{0, r}-\epsilon \phi^{0, r}$, with $\epsilon \sim v_{S M}^{3} /\left[\left(m_{\chi^{0}}^{2}-m_{h}^{2}\right) M_{P l}\right]$. The neutral member of $X$ can then decay through its Higgs component. Assuming that $\chi^{0}$ is the lightest state, its lifetime will range from a few days to a few years for $m_{\chi^{0}} \sim 100-1000 \mathrm{GeV}$. This puts the decays of the lightest neutral state of the $n=5$ model after big-bang nucleosynthesis but before neutral hydrogen atoms formed in 
the recombination period. As a result, direct search constraints from present-day experiments do not apply to this model, and it remains viable [125]. We therefore only consider the effect of dark matter constraints on the $n=6,7,8$ models.

\section{Limits from direct searches at the XENON-100 experiment}

As $\chi^{0}$ is a complex scalar, it scatters off nucleons via both $Z$ and Higgs exchange. The $Z$-exchange diagram yields a large scattering cross section, leading to very stringent constraints on $\Omega_{\chi} / \Omega_{\mathrm{DM}}$. The cross sections are computed in the zero-velocity limit assuming equal densities of $\chi^{0}$ and $\chi^{0 *}$, such that $\Omega_{\chi} \equiv \Omega_{\chi^{0}}+\Omega_{\chi^{0 *}}$. The spinindependent cross section is

$$
\sigma_{\mathrm{SI}}^{\chi^{0}}=\sigma_{Z}^{\chi^{0}}+\sigma_{h}^{\chi^{0}}+\sigma_{\text {int }}^{\chi^{0}}
$$

where $\sigma_{\text {int }}^{\chi^{0}}$ is the interference between the $Z$ - and Higgs-exchange diagrams. For $\chi^{0 *}$ $\sigma_{Z, h}^{\chi^{0 *}}=\sigma_{Z, h}^{\chi^{0}}$ and $\sigma_{\text {int }}^{\chi^{0 *}}=-\sigma_{\text {int }}^{\chi^{0}}$, so the interference term cancels in the total scattering rate for equal densities of $\chi^{0}$ and $\chi^{0 *}$.

The cross-section contributions of Eq. (5.28) were calculated by collaborators Terry Pilkington and Heather Logan in Ref. [2]. The resulting upper limit on $\Omega_{\chi} / \Omega_{\mathrm{DM}}$ from XENON100 [118] as a function of the $\chi^{0}$ mass is shown in Figs. 5.2 and 5.3 for the multiplets with $n=6,7$, and 8 (the limit depends on $n$ like $\left.1 /(n-1)^{2}\right)$. The shaded area bounded by the red curve is excluded.

\section{Thermal relic density}

The thermal relic density given by Eq. (3.109) was also calculated by collaborators in Ref. [2], allowing for a comparison with the upper bound from XENON-100 using 


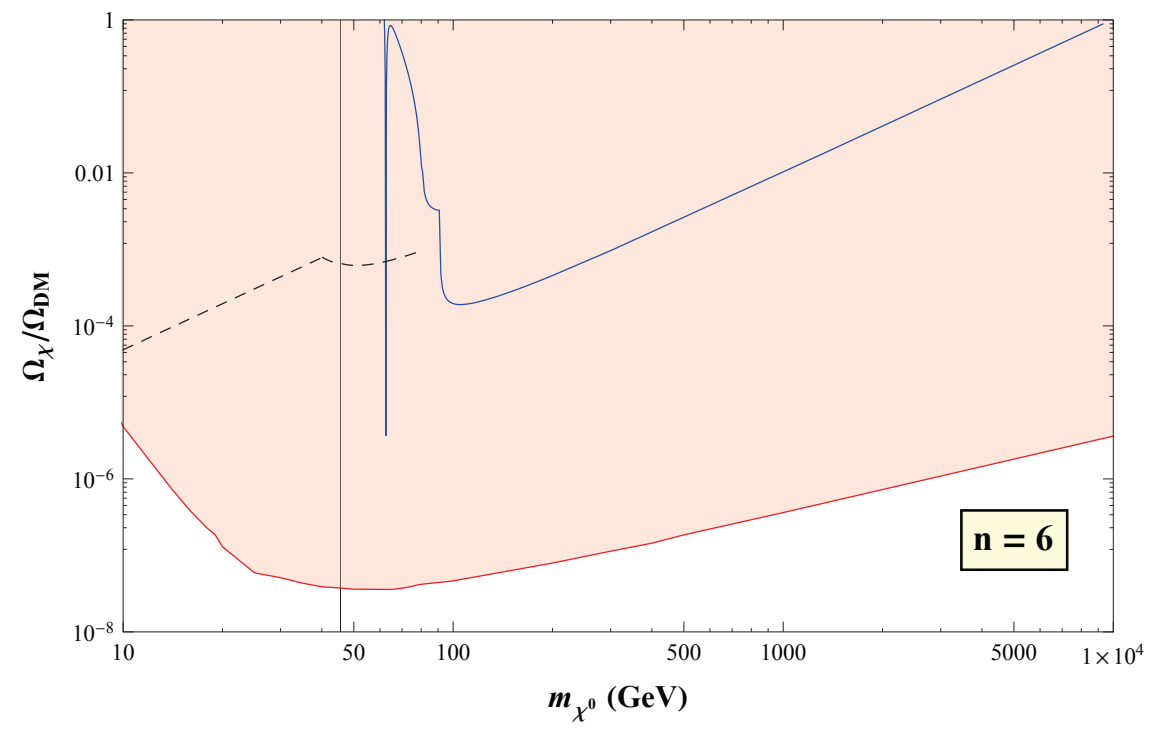

Figure 5.2: The fraction $\Omega_{\chi} / \Omega_{\mathrm{DM}}$ of the total dark matter density for the $n=6$ model as a function of $m_{\chi^{0}}$. The shaded area above the red curve is excluded by directdetection constraints from XENON-100 data [118], conservatively taking $\Lambda_{0}=0$. The solid blue curve shows the predicted relic density assuming a standard thermal history of the universe, for the parameters $\Lambda_{0}=0.01, \lambda_{3}=-0.01$. The black dashed curve is the relic density in the case that coannihilations are maximal. Masses below $m_{Z} / 2$ (to the left of the vertical black line) are excluded by the LEP constraint on the invisible $Z$ width.
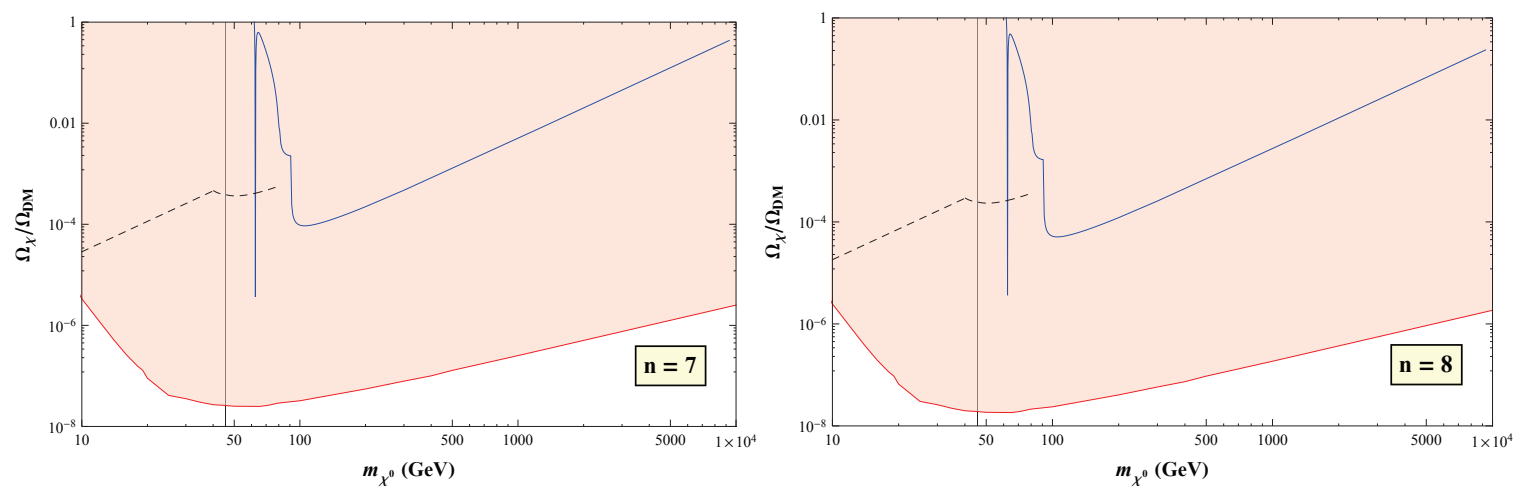

Figure 5.3: The same as Fig. 5.2 but for $n=7$ (left) and 8 (right). 
Eq. (3.110). The result is shown by the solid blue line in Figs. 5.2 and 5.3. The calculation assumes a standard thermal history of the universe as described in Sec. 3.2.6. Above the $W W$ threshold of $m_{\chi}=m_{W} \simeq 80 \mathrm{GeV}, \chi^{0 *} \chi^{0}$ annihilations to two-body final states $W W, Z Z, h h$ and $f \bar{f}$ dominate; analytical formulas for these annihilation cross sections are available in Ref. [2]. Below the $W W$ threshold, the largest contribution from two-body decays comes from $\chi^{0 *} \chi^{0} \rightarrow b \bar{b}$, which is reflected by the sharp bump in the blue line over that region. The dashed grey line accounts for the possibility that equal abundances of each $\chi^{Q}$ species at freeze-out ("coannihilation") result in a preferential annihilation to $\gamma \gamma, Z \gamma$, or $W^{ \pm} \gamma$, resulting in a suppression of the relic abundance.

One will note that in general these thermal relic densities are all well above the upper limits from XENON-100. However, there is a sharp resonant feature in the blue line at $m_{h} / 2 \simeq 62.5 \mathrm{GeV}$ due to resonant annihilation on the Higgs pole, which risks dipping below the exclusion bound. On the resonance the dominant contribution to $\Omega_{\chi} / \Omega_{\mathrm{DM}}$ arises from $\chi^{0} \chi^{0 *} \rightarrow h \rightarrow b \bar{b}$, which is proportional to $\Lambda_{0}^{2}$. This resonant behaviour is plotted in Fig. 5.4. One will note, for example, that $\left|\Lambda_{0}\right| \gtrsim 0.09$ is required to reduce the relic abundance enough to evade the direct-detection limit for the $n=6$ model.

However, this parameter may be limited by using a combination of constraints from the oblique parameters and the $h \rightarrow \gamma \gamma$ decay width. Setting $m_{\chi^{0}}=m_{h} / 2$, the $S, T$ and $U$ parameters put an upper bound on $\left|\lambda_{3}\right|$, as summarized in Table 5.2. For $\lambda_{3}$ within each allowed range, we compute the partial width $\Gamma(h \rightarrow \gamma \gamma)$ using Eq. (5.25). This partial width normalized to its SM value is plotted as a function of $\Lambda_{0}$ in Fig. 5.5 for the two extreme cases of $\lambda_{3}=0$ and $\lambda_{3}^{\max }$ from $S, T$ and $U$. As the decay width increases rapidly with $\left|\Lambda_{0}\right|, \Gamma(h \rightarrow \gamma \gamma)$ quickly exceeds the experimental 


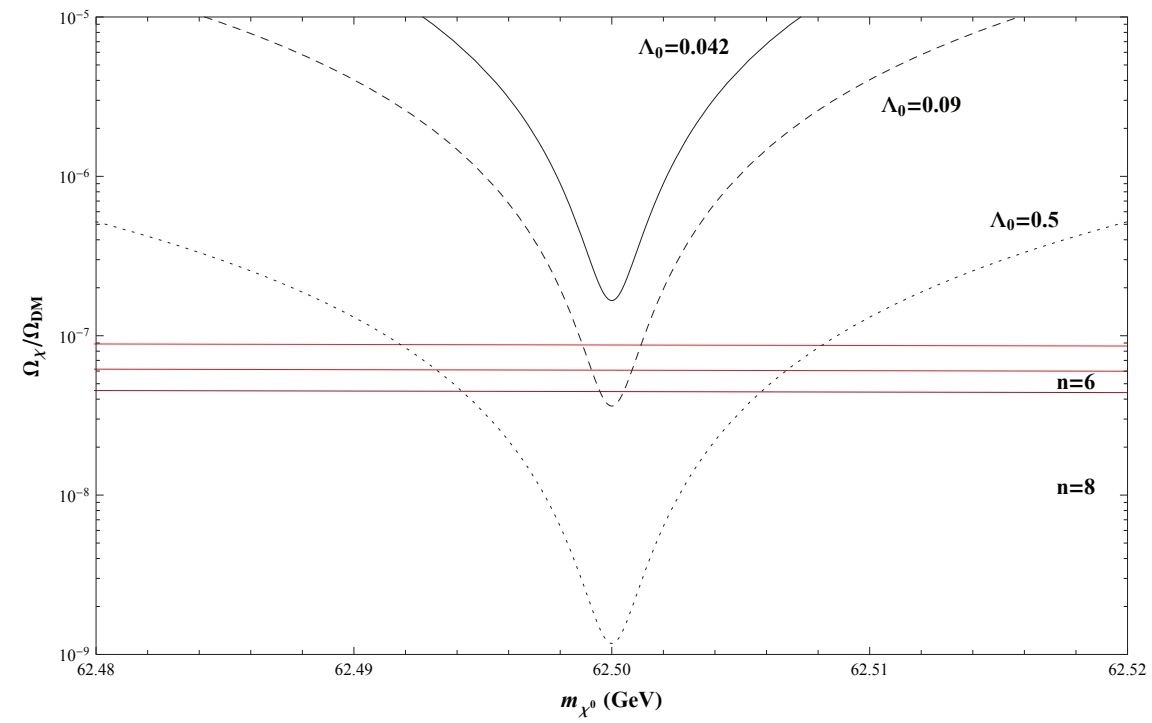

Figure 5.4: Fractional relic density $\Omega_{\chi} / \Omega_{\mathrm{DM}}$ for $m_{\chi^{0}} \simeq m_{h} / 2$ from $\chi^{0} \chi^{0 *} \rightarrow b \bar{b}$ annihilation, for various values of $\Lambda_{0}$ (solid, dashed, and dotted black curves). Also shown are the upper limits on $\Omega_{\chi} / \Omega_{\mathrm{DM}}$ from direct detection for (top to bottom) $n=6,7$, and 8 (red horizontal lines).
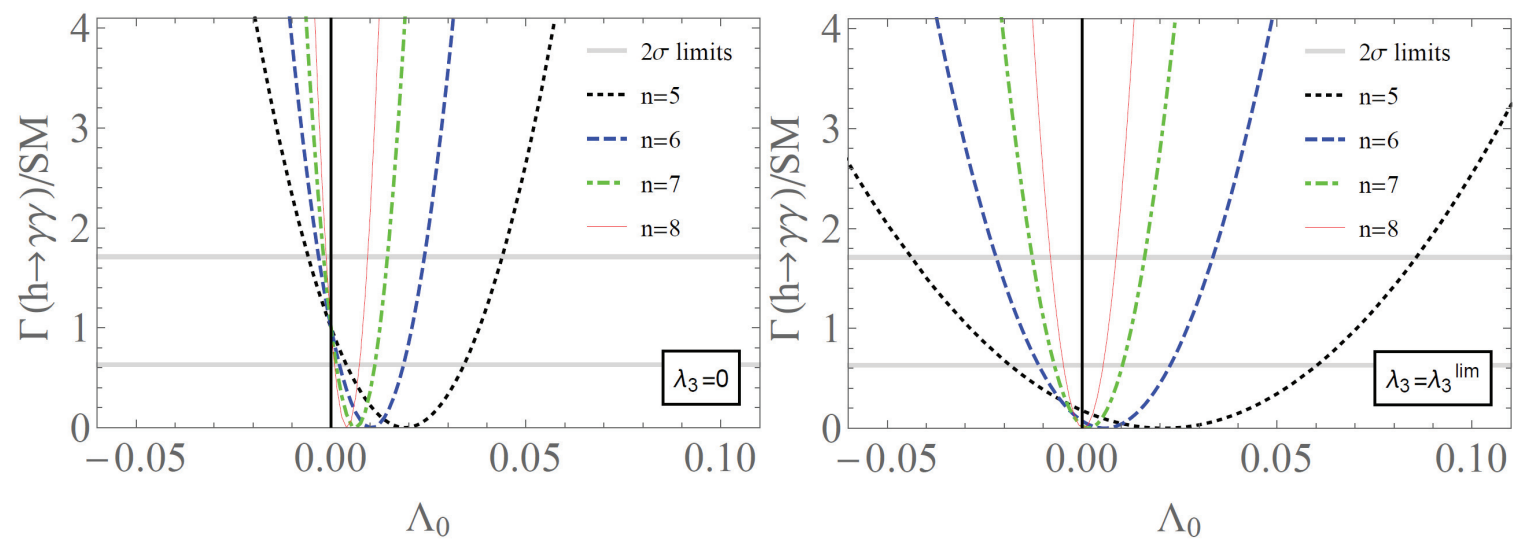

Figure 5.5: The partial width for $h \rightarrow \gamma \gamma$ normalized to its SM value as a function of $\Lambda_{0}$, for $m_{\chi^{0}}=m_{h} / 2=62.5 \mathrm{GeV}$ and $\lambda_{3}=0$ (left) and the limiting (minimum) value allowed by the oblique parameter constraints as given in Table 5.2 (right). The gray horizontal lines indicate the $2 \sigma$ experimental bounds on $h \rightarrow \gamma \gamma$ from ATLAS. 


\begin{tabular}{|c|cccc|}
\hline$n$ & 5 & 6 & 7 & 8 \\
\hline$\lambda_{3}^{\lim }$ & $-1.8 \times 10^{-2}$ & $-7.8 \times 10^{-3}$ & $-4.0 \times 10^{-3}$ & $-2.2 \times 10^{-3}$ \\
\hline
\end{tabular}

Table 5.2: The $95 \%$ C.L. lower limit on $\lambda_{3}$ from the $S, T$, and $U$ constraints for $5 \leq n \leq 8$ and $m_{\chi^{0}}=m_{h} / 2=62.5 \mathrm{GeV}$.

bounds imposed by results from the ATLAS and CMS experiments (summarized in Table 3.1 and represented by gray horizontal lines in Fig. 5.5). As a result, the allowed values of $\Lambda_{0}$ are too small to evade the direct-detection limit.

In particular, for $n=6$ the $h \rightarrow \gamma \gamma$ bound requires $\left|\Lambda_{0}\right|<0.046$ even if we accept decay widths up to three times the size of the SM prediction (which is already excluded at more than $2 \sigma$ by both ATLAS and CMS $[126,127])$. The value required to evade the direct-detection limit for the $n=6$ model, $\left|\Lambda_{0}\right| \gtrsim 0.09$, is therefore solidly excluded by this constraint. As these limits only become more stringent as $n$ increases, resonant annihilation through the Higgs pole cannot suppress $\Omega_{\chi} / \Omega_{\mathrm{DM}}$ enough to evade the direct-detection limits for $n=6,7$, or 8 .

Regardless of the model parameters, we conclude that for all parameter values the thermal relic abundance of $\chi^{0(*)}$ is too large to be consistent with the upper bound on $\Omega_{\chi} / \Omega_{\mathrm{DM}}$ from direct detection. The $\mathrm{U}(1)$-preserving models with $n=6,7$, and 8 are thus excluded assuming a standard thermal history of the universe. The $n=5$ models remain viable due to the ability of the lightest neutral scalar to decay; we leave further study of these models to future work. 


\section{Chapter 6}

\section{Models with a $\mathrm{Z}_{2}$ symmetry}

We now consider the models with additional large $(n \geq 5)$ scalar multiplets that preserve an additional $Z_{2}$ symmetry in the scalar potential. As before, this additional symmetry leads to a dark matter candidate, which may be required to be neutral with an appropriate choice of hypercharge. These models can be divided into two categories: those with real multiplets, and those with complex multiplets. The $Z_{2^{-}}$ symmetric models with an additional real $(Y=0)$ multiplet of size $n=5,7,9^{1}$ (isospin $T=2,3,4$ ) have previously been considered in the literature $[14,15]$, and have been found to produce viable dark matter candidates.

We therefore focus on the unstudied $Z_{2}$-symmetric models that include complex multiplets. In the case that our $5 \leq n \leq 8$ complex multiplets have $Y=1$ (as opposed to $Y=2 T$ as in Chap. 5), an additional term is permitted in the scalar potential which allows the global $U(1)$ symmetry considered in the previous chapter to be broken to $Z_{2}$. This additional symmetry breaking causes mixing between the BSM scalar states, resulting in very different phenomenology from the $U(1)$ models.

\footnotetext{
${ }^{1}$ Recall that real multiplets are required to have integer isospin $T$, and therefore $n$ is odd.
} 


\subsection{Model description}

In these models, the most general gauge-invariant scalar potential that preserves $Z_{2}$ is given by

$$
\begin{aligned}
V(\Phi, Z)= & m^{2} \Phi^{\dagger} \Phi+M^{2} Z^{\dagger} Z+\lambda_{1}\left(\Phi^{\dagger} \Phi\right)^{2}+\lambda_{2} \Phi^{\dagger} \Phi Z^{\dagger} Z+\lambda_{3} \Phi^{\dagger} \tau^{a} \Phi Z^{\dagger} T^{a} Z \\
& +\left[\lambda_{4} \widetilde{\Phi}^{\dagger} \tau^{a} \Phi Z^{\dagger} T^{a} \widetilde{Z}+\text { h.c. }\right]+\mathcal{O}\left(Z^{4}\right)
\end{aligned}
$$

where $Z_{2}$ is the large scalar multiplet, $\Phi$ is the Higgs doublet (as defined in Eq. (5.1)), $\tau^{a}$ and $T^{a}$ are the generators of $\mathrm{SU}(2)_{L}$ in the doublet and $n$-plet representations, and $\widetilde{x}=C x^{*}$ are the conjugate multiplets. The conjugation matrix, $C$, is an antisymmet-

ric $n \times n$ matrix. It is equal to $i \sigma^{2}$ for the $\mathrm{SU}(2)_{L}$ doublet (where $\sigma^{2}$ is the second Pauli matrix), while for the $n=6$ and $n=8$ representations it is given by

$$
C_{(n=6)}=\left(\begin{array}{cccccc}
0 & 0 & 0 & 0 & 0 & 1 \\
0 & 0 & 0 & 0 & -1 & 0 \\
0 & 0 & 0 & 1 & 0 & 0 \\
0 & 0 & -1 & 0 & 0 & 0 \\
0 & 1 & 0 & 0 & 0 & 0 \\
-1 & 0 & 0 & 0 & 0 & 0
\end{array}\right), \quad C_{(n=8)}=\left(\begin{array}{cccccccc}
0 & 0 & 0 & 0 & 0 & 0 & 0 & 1 \\
0 & 0 & 0 & 0 & 0 & 0 & -1 & 0 \\
0 & 0 & 0 & 0 & 0 & 1 & 0 & 0 \\
0 & 0 & 0 & 0 & -1 & 0 & 0 & 0 \\
0 & 0 & 0 & 1 & 0 & 0 & 0 & 0 \\
0 & 0 & -1 & 0 & 0 & 0 & 0 & 0 \\
0 & 1 & 0 & 0 & 0 & 0 & 0 & 0 \\
-1 & 0 & 0 & 0 & 0 & 0 & 0 & 0
\end{array}\right) .
$$

Eq. (6.1) is not the most general scalar potential allowed for the multiplet $Z$. In order to preserve the desired global $Z_{2}$ symmetry and avoid phenomenologically dangerous contributions to the $\rho$ parameter from a nonzero vev of $Z$, we have forbidden the $Z_{2}$-violating term involving the field combination $Z^{\dagger} Z^{\dagger} Z \Phi$. In Eq. (6.1) we do not display the explicit form of the $Z$ quartic scalar couplings; similarly to the $U(1)$ case in the previous chapter, they are not required in the work that follows as we may obtain conservative bounds on the potential parameters without them. 
The term proportional to $\lambda_{4}$ in Eq. (6.1) is not present in the models with $Y=2 T$ considered in the previous chapter. This term couples two SM Higgs fields $\Phi$ (not $\Phi^{*}$ ) to two $Z^{*}$ fields, with each pair arranged in an isospin-triplet configuration with total hypercharge \pm 2 . This term breaks the global $U(1)$ symmetry down to $Z_{2}$ and splits the masses of the real and imaginary components of the neutral member of $Z, \zeta^{0} \equiv\left(\zeta^{0, r}+i \zeta^{0, i}\right) / \sqrt{2}$.

The term $Z^{\dagger} T^{a} \widetilde{Z}$ is nonzero only for even $n$, and the perturbative unitarity constraint from Eq. (4.26) requires that $n \leq 8$. The $n=2$ and $n=4$ cases have previously been considered in the literature $[8,128]$; in fact, the addition of an $n=2$ $(T=1 / 2)$ doublet with $Y=1$ results in the Two Higgs Doublet model. As a result, only the models with $n=6(T=5 / 2)$ and $n=8(T=7 / 2)$ are of interest to us in the $Z_{2}$-symmetric case. For these cases, the large multiplet is given in the electroweak basis by

$$
\begin{aligned}
& Z_{(n=6)}=\left(\zeta^{+3}, \zeta^{+2}, \zeta^{+1}, \zeta^{0}, \zeta^{-1}, \zeta^{-2}\right)^{T} \\
& Z_{(n=8)}=\left(\zeta^{+4}, \zeta^{+3}, \zeta^{+2}, \zeta^{+1}, \zeta^{0}, \zeta^{-1}, \zeta^{-2}, \zeta^{-3}\right)^{T} .
\end{aligned}
$$

We will denote the conjugate of the charged state $\zeta^{Q}$ by $\zeta^{Q *}$. Note that $\zeta^{-Q} \neq \zeta^{Q *}$.

\subsubsection{Particle spectrum and masses}

The presence of the $\lambda_{4}$ term induces mixing among the scalar states $\zeta$ and a mass splitting between the real and imaginary components of the neutral state $\zeta^{0}$. We may take $\lambda_{4}$ to be real, as an additional complex phase may be rotated away through a redefinition of $Z$. Working in unitarity gauge, the term involving $\lambda_{4}$ in the scalar potential of Eq. (6.1) reduces to

$$
\lambda_{4} \widetilde{\Phi}^{\dagger} \tau^{a} \Phi Z^{\dagger} T^{a} \widetilde{Z}+\text { h.c. }=\frac{1}{4} \lambda_{4}(h+v)^{2}\left[Z^{\dagger} T^{-} \widetilde{Z}+\widetilde{Z}^{\dagger} T^{+} Z\right],
$$


where $T^{ \pm}=T^{1} \pm i T^{2}$. The terms $Z^{\dagger} T^{-} \widetilde{Z}$ and $\widetilde{Z}^{\dagger} T^{+} Z$ split the masses of $\zeta^{0, r}$ and $\zeta^{0, i}$ and cause mixing between states with the same electric charge but different isospin. For $n=6,8$, the two pieces can be written as

$$
\begin{aligned}
& Z^{\dagger} T^{-} \widetilde{Z}=\frac{n}{2}(-1)^{n / 2+1} \zeta^{0 *} \zeta^{0 *}+\sum_{Q=1}^{n-1} \sqrt{n^{2}-4 Q^{2}}(-1)^{n / 2+Q+1} \zeta^{+Q *} \zeta^{-Q *}, \\
& \widetilde{Z}^{\dagger} T^{+} Z=\frac{n}{2}(-1)^{n / 2+1} \zeta^{0} \zeta^{0}+\sum_{Q=1}^{n-1} \sqrt{n^{2}-4 Q^{2}}(-1)^{n / 2+Q+1} \zeta^{+Q} \zeta^{-Q}
\end{aligned}
$$

When the neutral state $\zeta^{0}$ is written in terms of its real and imaginary components, $\zeta^{0}=\left(\zeta^{0, r}+i \zeta^{0, i}\right) / \sqrt{2}$, we find a mass splitting between the components,

$$
\begin{aligned}
& m_{\zeta^{0, r}}^{2}=M^{2}+\frac{1}{2} v^{2}\left[\lambda_{2}+\frac{1}{4} \lambda_{3}+\frac{n}{2}(-1)^{n / 2+1} \lambda_{4}\right] \equiv M^{2}+\frac{1}{2} v^{2} \Lambda_{n}, \\
& m_{\zeta^{0, i}}^{2}=M^{2}+\frac{1}{2} v^{2}\left[\lambda_{2}+\frac{1}{4} \lambda_{3}+\frac{n}{2}(-1)^{n / 2} \lambda_{4}\right]=m_{\zeta^{0, r}}^{2}+\frac{n}{2}(-1)^{n / 2} v^{2} \lambda_{4} .
\end{aligned}
$$

As discussed earlier, we require that the lightest member of these multiplets be electrically neutral. Without loss of generality, we choose the real part of $\zeta^{0}$ to be the lightest member of the multiplet, corresponding to a choice of the sign of $\lambda_{4}$. We can see from Eqs. (6.7-6.8) that the sign of $\lambda_{4}$ will depend on $n$, due to the factors of $(-1)^{n / 2}$. The requirement that $m_{\zeta^{0, r}} \leq m_{\zeta^{0, i}}$ imposes

$$
\lambda_{4}<0 \text { for } n=6 \quad \text { and } \quad \lambda_{4}>0 \text { for } n=8 .
$$

The mass matrices for the pairs of scalars with electric charge $Q=1, \ldots, n / 2-$ 1 are given in the basis $\left(\zeta^{+Q}, \zeta^{-Q *}\right)$ by

$$
M_{\zeta^{ \pm Q}}^{2}=\left(\begin{array}{ll}
M^{2}+\frac{1}{8} v^{2}\left(4 \lambda_{2}-(2 Q-1) \lambda_{3}\right) & \frac{1}{4} v^{2} \lambda_{4} \sqrt{n^{2}-4 Q^{2}}(-1)^{n / 2+Q+1} \\
\frac{1}{4} v^{2} \lambda_{4} \sqrt{n^{2}-4 Q^{2}}(-1)^{n / 2+Q+1} & M^{2}+\frac{1}{8} v^{2}\left(4 \lambda_{2}+(2 Q+1) \lambda_{3}\right)
\end{array}\right),
$$

which we diagonalize to find the mass eigenvalues,

$$
\begin{aligned}
m_{H_{1,2}^{Q}}^{2} & =M^{2}+\frac{1}{2} v^{2}\left(\lambda_{2}+\frac{1}{4} \lambda_{3} \mp \frac{1}{2} \sqrt{Q^{2} \lambda_{3}^{2}+\left(n^{2}-4 Q^{2}\right) \lambda_{4}^{2}}\right) \\
& =m_{\zeta^{0, r}}^{2}+\frac{1}{4} v^{2}\left(n(-1)^{n / 2} \lambda_{4} \mp \sqrt{Q^{2} \lambda_{3}^{2}+\left(n^{2}-4 Q^{2}\right) \lambda_{4}^{2}}\right) .
\end{aligned}
$$


The mass eigenstates are defined for $Q>0$ in terms of mixing angles $\alpha_{Q}$ as

$$
\begin{aligned}
& H_{1}^{Q}=\cos \alpha_{Q} \zeta^{Q}+\sin \alpha_{Q} \zeta^{-Q *}, \\
& H_{2}^{Q}=-\sin \alpha_{Q} \zeta^{Q}+\cos \alpha_{Q} \zeta^{-Q *},
\end{aligned}
$$

where we have defined $H_{1}^{Q}$ as the lighter state and $H_{2}^{Q}$ as the heavier state, $m_{H_{1}^{Q}}<$ $m_{H_{2}^{Q}}$. The mixing angle $\alpha_{Q} \in\left[-\frac{\pi}{2}, \frac{\pi}{2}\right]$ is given by

$$
\begin{aligned}
\tan \alpha_{Q} & =(-1)^{n / 2+Q+1} \frac{Q \lambda_{3}-\sqrt{Q^{2} \lambda_{3}^{2}+\left(n^{2}-4 Q^{2}\right) \lambda_{4}^{2}}}{\sqrt{\left(n^{2}-4 Q^{2}\right) \lambda_{4}^{2}}} \\
& =(-1)^{n / 2+Q} \frac{\sqrt{n^{2}-4 Q^{2}} \lambda_{4}}{Q \lambda_{3}+\sqrt{Q^{2} \lambda_{3}^{2}+\left(n^{2}-4 Q^{2}\right) \lambda_{4}^{2}}} .
\end{aligned}
$$

Recalling the bounds on $\lambda_{4}$ from Eq. (6.9), one will note that the $H_{2}^{Q}$ states will always be heavier than the neutral real state $\zeta^{0, r}$, as all of the additional mass contributions are positive for the appropriate sign of $\lambda_{4}$. In addition, Eq. (6.9) ensures that all of the $H_{2}$ states have larger masses than all of the $H_{1}$ states, since the only difference between the two definitions in Eq. (6.11) is the subtraction versus the addition of positive terms,

$$
m_{H_{1}^{Q_{i}}}^{2}<m_{\zeta^{0, r}}^{2}+\frac{(-1)^{n / 2}}{4} v^{2} n \lambda_{4}<m_{H_{2}^{Q_{j}}}^{2} \quad \text { for all } Q_{i, j} .
$$

However, in order for $m_{\zeta^{0, r}} \leq m_{H_{1}^{Q}}$ we require that the total sum of the bracketed terms in Eq. (6.11) be larger than zero. This requires that

$$
\left|\lambda_{3}\right| \leq 2\left|\lambda_{4}\right| \text { for all } n \text {. }
$$

Under this relation between $\lambda_{3}$ and $\lambda_{4}$, the quantity under the square root in Eq. (6.11) - which may be written as $n^{2} \lambda_{4}^{2}-Q^{2}\left(4 \lambda_{4}^{2}-\lambda_{3}^{2}\right)$ - will decrease with $Q$. As a result, the masses of the $H_{1}^{Q}$ states increase with $Q$, while the masses of the $H_{2}^{Q}$ states decrease with $Q$. Eq. (6.16) also ensures that the neutral imaginary state $\zeta^{0, i}$ will 
always be heavier than or equal in mass to the $H_{2}^{Q}$ states, as the square root term will always be smaller than or equal to $(-1)^{n / 2} v^{2} n \lambda_{4} / 4$.

There is only one state with $Q=n / 2$. Its mass is given by

$$
m_{\zeta^{n / 2}}^{2}=M^{2}+\frac{1}{8} v^{2}\left(4 \lambda_{2}-(2 Q-1) \lambda_{3}\right)=m_{\zeta^{0, r}}^{2}+\frac{(-1)^{n / 2}}{4} v^{2} n \lambda_{4}-\frac{n}{8} v^{2} \lambda_{3} .
$$

With Eq. (6.16), the term $n v^{2} \lambda_{3} / 8$ will always be smaller in magnitude than the square root term in Eq. (6.11); they become equal in the limit that $\left|\lambda_{3}\right|=2\left|\lambda_{4}\right|$ and $Q=n / 2-1$. Therefore $\zeta^{n / 2}$ will always be lighter or equal in mass to the $H_{2}^{Q}$ states, but heavier than the $H_{1}^{Q}$ states.

As a result, the overall mass hierarchy of the particle mass states — from lightest to heaviest - is as follows,

$$
\zeta^{0, r}, H_{1}^{+}, H_{1}^{++}, \ldots, H_{1}^{n / 2-1}, \zeta^{n / 2}, H_{2}^{n / 2-1}, \ldots, H_{2}^{++}, H_{2}^{+}, \zeta^{0, i}
$$

This spectrum is illustrated as a function of $\lambda_{4}$ in Fig. 6.1 for two sample points: $\left\{n=6, m_{\zeta^{0, r}}=150, \lambda_{3}=-0.2\right\}$ and $\left\{n=6, m_{\zeta^{0, r}}=500 \mathrm{GeV}, \lambda_{3}=-3\right\}$. The result is similar in structure for $n=8$.

\subsubsection{Degeneracy and clustering in the mass spectrum}

Fig. 6.1 illustrates a point of degeneracy in the mass spectrum with a dotted vertical line, which arises when Eq. (6.16) becomes an equality. In the limit that $\left|\lambda_{3}\right|=\left|2 \lambda_{4}\right|$, the mass spectrum collapses to two degenerate sets of particles: a group of mass $m_{\zeta^{0, r}}$

containing the $\zeta^{0, r}$ and $H_{1}^{Q}$ states, and group of mass $m_{\zeta^{0, i}}$ containing the $\zeta^{n / 2}, H_{2}^{Q}$, and $\zeta^{0, i}$ states,

$$
\left\{\begin{array}{c}
m_{\zeta^{0, r}}=m_{H_{1}^{Q}} \\
m_{\zeta^{0, i}}=m_{H_{2}^{Q}}=m_{\zeta^{n / 2}}=\sqrt{m_{\zeta^{0, r}}^{2}+n v^{2}\left|\lambda_{4}\right| / 2}
\end{array}\right\} \quad \forall n, Q \text { if }\left|\lambda_{3}\right|=2\left|\lambda_{4}\right| .
$$



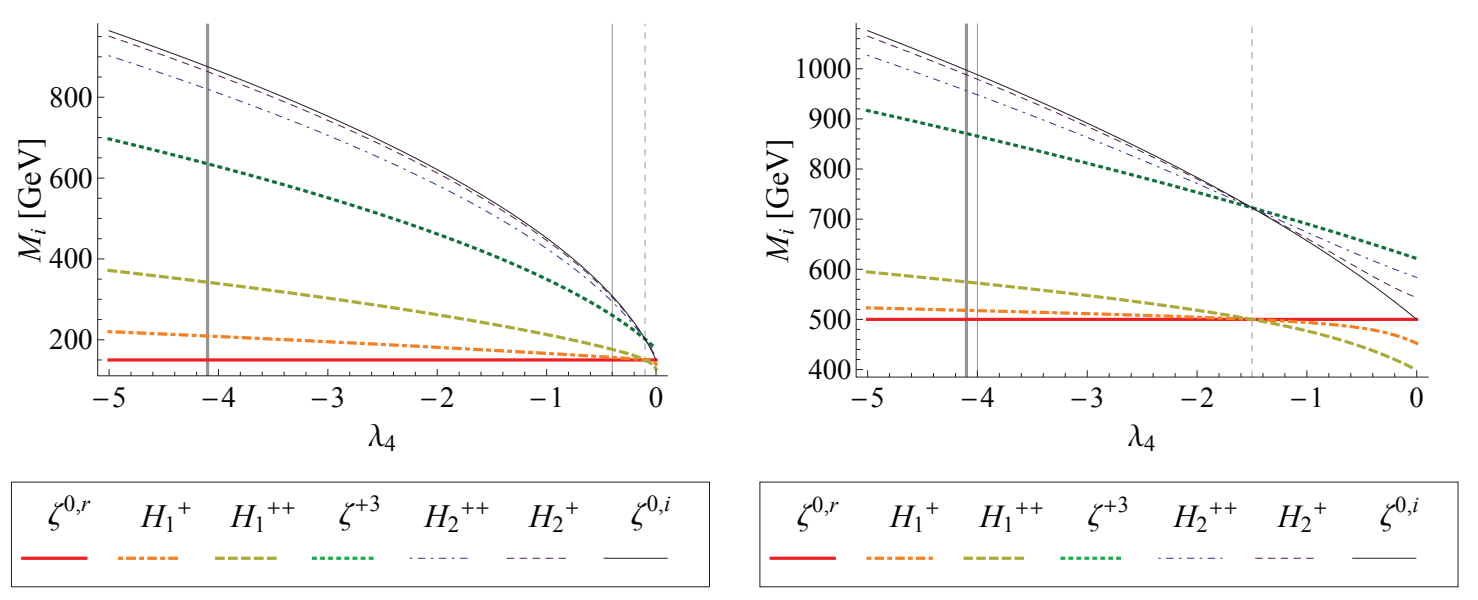

Figure 6.1: Sample mass spectra for the $n=6$ model as a function of $\lambda_{4}$. Allowed $\lambda_{4}$ values lie to the left of the vertical dotted line (so that $\zeta^{0, r}$ is the lightest state) and to the right of both vertical solid lines. The thick vertical solid line marks the unitarity bound on $\lambda_{4}$ while the thin vertical solid line marks the bound from precision electroweak constraints. Left: $m_{\zeta^{0, r}}=150 \mathrm{GeV}$ and $\lambda_{3}=-0.2$. Right: $m_{\zeta^{0, r}}=$ $500 \mathrm{GeV}$ and $\lambda_{3}=-3$.

The mass states are not permitted to deviate very far from this degenerate scenario. Even when the masses are no longer degenerate, the states remain clustered in two similar groups of low mass $\zeta^{0, r}, H_{1}^{Q}$ and high mass $\zeta^{n / 2}, H_{2}^{Q}, \zeta^{0, i}$. This clustering of states arises due to bounds on $\lambda_{3}$ and $\lambda_{4}$ from the oblique parameters. As we will see in Sec. 6.3.2, constraints from these parameters favour $\lambda_{3}<0$ and require that $\left|\lambda_{3}\right|$ be very close to $\left|2 \lambda_{4}\right|$. The mass splitting between the members of each group is effectively controlled by $\lambda_{3}$, while the mass gap between the two groups is controlled by $\lambda_{4}$; this behaviour is illustrated in Fig. 6.1.

\subsubsection{Model summaries}

\section{Summary of the $n=6$ model}

In the $n=6$ model, we require that $\lambda_{4}<0$ and $\left|\lambda_{3}\right| \leq 2\left|\lambda_{4}\right|$ for $\zeta^{0, r}$ to be the lightest scalar in the large multiplet $Z$. After mixing, the particle spectrum of the $n=6$ 
multiplet $Z$ given in Eq. 6.3 becomes, from lightest to heaviest,

$$
\zeta^{0, r}, H_{1}^{+}, H_{1}^{++}, \zeta^{+3}, H_{2}^{++}, H_{2}^{+}, \zeta^{0, i}
$$

with masses given by

$$
\begin{gathered}
m_{\zeta^{0, r}}^{2}=M^{2}+\frac{1}{2} v^{2}\left[\lambda_{2}+\frac{1}{4} \lambda_{3}+3 \lambda_{4}\right] \equiv M^{2}+\frac{1}{2} v^{2} \Lambda_{6}, \\
m_{\zeta^{0, i}}^{2}=m_{\zeta^{0, r}}^{2}-3 v^{2} \lambda_{4} \\
m_{H_{1,2}^{+}}^{2}=m_{\zeta^{0, r}}^{2}+\frac{1}{4} v^{2}\left(-6 \lambda_{4} \mp \sqrt{\lambda_{3}^{2}+32 \lambda_{4}^{2}}\right), \\
m_{H_{1,2}^{++}}^{2}=m_{\zeta^{0, r}}^{2}+\frac{1}{2} v^{2}\left(-3 \lambda_{4} \mp \sqrt{\lambda_{3}^{2}+5 \lambda_{4}^{2}}\right) \\
m_{\zeta^{+3}}^{2}=m_{\zeta^{0, r}}^{2}-\frac{3}{4} v^{2}\left(\lambda_{3}+2 \lambda_{4}\right)
\end{gathered}
$$

where for future reference we have defined the part of the scalar mass that arises from the Higgs boson as

$$
\Lambda_{6}=\lambda_{2}+\frac{1}{4} \lambda_{3}+3 \lambda_{4}
$$

The mixing angles $\alpha_{Q}$ for the mass eigenstates $H_{1,2}^{Q}$ are given by

$$
\begin{aligned}
\tan \alpha_{1} & =\frac{4 \sqrt{2} \lambda_{4}}{\lambda_{3}+\sqrt{\lambda_{3}^{2}+32 \lambda_{4}^{2}}}, \\
\tan \alpha_{2} & =\frac{-\sqrt{5} \lambda_{4}}{\lambda_{3}+\sqrt{\lambda_{3}^{2}+5 \lambda_{4}^{2}}} .
\end{aligned}
$$

In the limit $\left|\lambda_{3}\right|=2\left|\lambda_{4}\right|$, the mass spectrum collapses to two degenerate sets of particles: $\zeta^{0, r}, H_{1}^{+}$, and $H_{1}^{++}$become degenerate with mass $m_{\zeta^{0, r}}$, while $\zeta^{+3}, H_{2}^{++}$, $H_{2}^{+}$, and $\zeta^{0, i}$ become degenerate with with mass $m_{\zeta^{0, i}}=\sqrt{m_{\zeta^{0, r}}^{2}-3 v^{2} \lambda_{4}}$.

\section{Summary of the $n=8$ model}

In the $n=8$ model, we require that $\lambda_{4}>0$ and $\left|\lambda_{3}\right| \leq 2\left|\lambda_{4}\right|$ for $\zeta^{0, r}$ to be the lightest scalar in the large multiplet $Z$. After mixing, the particle spectrum of the $n=8$ 
multiplet $Z$ given in Eq. (6.4) becomes, from lightest to heaviest,

$$
\zeta^{0, r}, H_{1}^{+}, H_{1}^{++}, H_{1}^{+3}, \zeta^{+4}, H_{2}^{+3}, H_{2}^{++}, H_{2}^{+}, \zeta^{0, i}
$$

with masses

$$
\begin{gathered}
m_{\zeta^{0, r}}^{2}=M^{2}+\frac{1}{2} v^{2}\left[\lambda_{2}+\frac{1}{4} \lambda_{3}-4 \lambda_{4}\right] \equiv M^{2}+\frac{1}{2} v^{2} \Lambda_{8} \\
m_{\zeta^{0, i}}^{2}=m_{\zeta^{0, r}}^{2}+4 v^{2} \lambda_{4} \\
m_{H_{1,2}^{+}}^{2}=m_{\zeta^{0, r}}^{2}+\frac{1}{4} v^{2}\left(8 \lambda_{4} \mp \sqrt{\lambda_{3}^{2}+60 \lambda_{4}^{2}}\right) \\
m_{H_{1,2}^{++}}^{2}=m_{\zeta^{0, r}}^{2}+\frac{1}{2} v^{2}\left(4 \lambda_{4} \mp \sqrt{\lambda_{3}^{2}+12 \lambda_{4}^{2}}\right) \\
m_{H_{1,2}^{+3}}^{2}=m_{\zeta^{0, r}}^{2}+\frac{1}{4} v^{2}\left(8 \lambda_{4} \mp \sqrt{9 \lambda_{3}^{2}+28 \lambda_{4}^{2}}\right) \\
m_{\zeta^{+4}}^{2}=m_{\zeta^{0, r}}^{2}-v^{2}\left(\lambda_{3}-2 \lambda_{4}\right)
\end{gathered}
$$

where for future reference we have defined the part of the scalar mass that arises from the Higgs boson as

$$
\Lambda_{8}=\lambda_{2}+\frac{1}{4} \lambda_{3}-4 \lambda_{4}
$$

The mixing angles $\alpha_{Q}$ of the mass eigenstates $H_{1,2}^{Q}$ are defined as

$$
\begin{aligned}
\tan \alpha_{1} & =\frac{-2 \sqrt{15} \lambda_{4}}{\lambda_{3}+\sqrt{\lambda_{3}^{2}+60 \lambda_{4}^{2}}}, \\
\tan \alpha_{2} & =\frac{2 \sqrt{3} \lambda_{4}}{\lambda_{3}+\sqrt{\lambda_{3}^{2}+12 \lambda_{4}^{2}}}, \\
\tan \alpha_{3} & =\frac{-2 \sqrt{7} \lambda_{4}}{3 \lambda_{3}+\sqrt{9 \lambda_{3}^{2}+28 \lambda_{4}^{2}}} .
\end{aligned}
$$

In the limit that $\left|\lambda_{3}\right|=2\left|\lambda_{4}\right|$, the mass spectrum again collapses to two degenerate sets of particles: $\zeta^{0, r}, H_{1}^{+}, H_{1}^{++}$, and $H_{1}^{+3}$ become degenerate with mass $m_{\zeta^{0, r}}$, while $\zeta^{+4}, H_{2}^{+3}, H_{2}^{++}, H_{2}^{+}$, and $\zeta^{0, i}$ become degenerate with mass $m_{\zeta^{0, i}}=\sqrt{m_{\zeta^{0, r}}^{2}+4 v^{2} \lambda_{4}}$. 


\subsection{Theoretical constraints}

For this class of models we will consider only theoretical constraints on the scalar potential parameters from tree-level perturbative unitarity. Although some of the parameter space in this class of models remains after our analysis, the models are strongly constrained by unitarity and experimental constraints without considering quartic interactions in the BSM scalars $\zeta^{Q}$. As a result, we do not consider constraints from the boundedness of the potential or alternative vacua in detail; for our purposes a sufficient condition to avoid alternate minima in which one or more of the $\zeta$ fields acquires a vev is to require $M^{2}>0$ in Eq. (6.1) [3]. We leave these additional constraints on the potential to future work.

\subsubsection{Constraints from tree-level perturbative unitarity}

As in the previous two chapters, the scalar quartic couplings $\lambda_{2}, \lambda_{3}$ and $\lambda_{4}$ given in Eq. (6.1) can be bounded by requiring tree-level perturbative unitarity of the zeroth partial wave scattering amplitudes according to Eq. (3.2). Again, we calculate the $S S \rightarrow S S, V V$ amplitudes in the high-energy limit, including Goldstone bosons in the place of longitudinal gauge boson polarizations. For the same reasons as in the $U(1)$ case we neglect contributions from $\lambda_{1}$, the interactions that are quartic in $Z$,

and any $S S \rightarrow V V$ amplitudes that are not enhanced by the multiplet size $n$. The resulting bounds are conservative.

The only difference between the Lagrangians of the $U(1)$ and $Z_{2}$ cases Eqs. (5.2) and (6.1) respectively - is the term proportional to $\lambda_{4}$, which mediates interactions of total hypercharge 2. Therefore the $Z_{2}$ tree level amplitudes of total hypercharge-0 with isospin-0 and isospin-1 will be identical to those of the $U(1)$ 
models, except that in the $\zeta^{*} \zeta$ amplitudes $Y=1$ rather than $Y=2 T$. In the $Z_{2}$ model the amplitudes, linear combinations of states, amplitude matrix, and eigenvalues for the $Q=0, T=\{0,1\}$ cases are given by Eqs. (5.6)-(5.11) $(T=0)$ and Eqs. (5.14)(5.18) $(T=1)$ with the replacements $\chi \rightarrow \zeta$ and $Y=1$. The resulting bounds on $\lambda_{2}$ and $\lambda_{3}$ are given in Eqs. (5.12) and (5.19). For $Y=1$, these become

$$
\begin{aligned}
& \left|\lambda_{2}\right| \leq \sqrt{\frac{32 \pi^{2}}{n}-\frac{g^{4}\left(n^{2}-1\right)^{2}}{24}-\frac{g^{4} s_{W}^{4}}{8 c_{W}^{4}}}, \\
& \left|\lambda_{3}\right| \leq 2 \sqrt{\frac{384 \pi^{2}}{n\left(n^{2}-1\right)}-g^{4} \frac{s_{W}^{2}}{c_{W}^{2}}} .
\end{aligned}
$$

Numerical $^{2}$ values for the bounds on $\lambda_{2}$ and $\lambda_{3}$ in the $n=6,8 Z_{2}$ models are tabulated in Table 6.1.

The new scalar potential parameter, $\lambda_{4}$, may be bounded by interactions with $T=1$ and $Y=2$. Only one amplitude contributes to this channel, given by

$$
\mathcal{M}_{0}\left([\zeta \zeta]_{1} \rightarrow[\phi \phi]_{1}\right)=-\frac{\sqrt{n\left(n^{2}-1\right)}}{\sqrt{6}} \lambda_{4}
$$

where the normalized isospin-one, hypercharge-two field combinations are

$$
\begin{aligned}
& {[\phi \phi]_{1}=\phi^{+} \phi^{0}} \\
& {[\zeta \zeta]_{1}=\sqrt{\frac{6}{n\left(n^{2}-1\right)}(-1)^{n / 2} \sum_{j=1}^{n / 2}(-1)^{j}(2 j-1) \zeta^{j} \zeta^{-j+1} .}}
\end{aligned}
$$

Applying Eq. (3.3) and the bound of Eq. (3.2), the unitarity constraint on $\lambda_{4}$ is

$$
\left|\lambda_{4}\right| \leq 8 \pi \sqrt{\frac{6}{n\left(n^{2}-1\right)}} .
$$

Numerical values for these $\lambda_{4}$ in bounds are also tabulated in Table 6.1.

\footnotetext{
${ }^{2}$ We use $g^{2}=4 \pi \alpha / s_{W}^{2}, s_{W}^{2}=0.231$, and $\alpha=1 / 128$.
} 


\begin{tabular}{llll}
\hline$n$ & $\left|\lambda_{2}\right|^{\max }$ & $\left|\lambda_{3}\right|^{\max }$ & $\left|\lambda_{4}\right|^{\max }$ \\
\hline 6 & 6.59 & 8.48 & 4.25 \\
8 & 3.10 & 5.46 & 2.74 \\
\hline
\end{tabular}

Table 6.1: Upper limits on $\left|\lambda_{2}\right|,\left|\lambda_{3}\right|$ and $\left|\lambda_{4}\right|$ from perturbative unitarity for the models with $n=6$ and 8 .

\subsection{Experimental constraints}

\subsubsection{Direct-detection constraints}

Due to the experimental bound from $Z$ decay at LEP, our lightest scalar $\xi^{0, r}$ is strongly bounded to be heavier than $45 \mathrm{GeV}$. However, in the work below we consider only

neutral scalar masses $m_{\zeta^{0, r}} \geq 80 \mathrm{GeV}$, as a proper assessment of the experimental bounds from LEP below the weak scale would require a dedicated collider study.

Very recently — following the publication of the work below — Ref. [129] has applied constraints to the $Z_{2}$ models using data from direct searches for supersymmetric electroweak gauginos at ATLAS and CMS. These bounds exclude masses $m_{\zeta^{0, r}}=80-120 \mathrm{GeV}$ in both models unless the mass splittings between the scalar states are below $20 \mathrm{GeV}$. However, the sensitivity of the bounds decreases above 120 $\mathrm{GeV}$ and becomes very weak for masses above $180 \mathrm{GeV}$. These new bounds should be kept in mind when considering results that vary with $m_{\zeta^{0, r}}$ (i.e. Figs. 6.6-6.11).

\subsubsection{Constraints from oblique parameters}

Further constraints can be obtained from experimental measurements of the electroweak oblique parameters $S, T$ and $U$ [84]. For the $Z_{2}$ models the $S$ parameter - 
originally defined in Eq. (3.31) — is given by

$$
S=\frac{2 s_{W}^{2} c_{W}^{2}}{\pi} \sum_{i, j}\left(\left|C_{i j Z}\right|^{2}-\frac{c_{W}^{2}-s_{W}^{2}}{s_{W} c_{W}} C_{i j Z} C_{i j \gamma}^{*}-\left|C_{i j \gamma}\right|^{2}\right) f_{1}\left(m_{i}, m_{j}\right)
$$

where the couplings $C_{i j V}$ involving scalars $i, j$ and vector boson $V$ are defined with an overall factor of $e$ removed (see Appendix A.2). The sums over states $i$ and $j$ run over $i j=\left\{\zeta^{0, r} \zeta^{0, i}, H_{k}^{+Q} H_{l}^{-Q}, \zeta^{n / 2} \zeta^{-n / 2}\right\}=\left\{I R, H_{k}^{Q} H_{l}^{-Q}, \zeta \zeta\right\}$, where $k l=11,12,21,22$, $Q>0$, and we have defined the additional shorthand notation for brevity. The dimensionless function $f_{1}\left(m_{1}, m_{2}\right)$ is defined by Eq. (3.39). Explicitly, we have

$$
\begin{aligned}
& S=\frac{2 s_{W}^{2} c_{W}^{2}}{\pi}\left[\left(\left|C_{\zeta \zeta Z}\right|^{2}-\frac{c_{W}^{2}-s_{W}^{2}}{c_{W} s_{W}} C_{\zeta \zeta Z} C_{\zeta \zeta \gamma}^{*}-\left|C_{\zeta \zeta \gamma}\right|^{2}\right) \frac{\ln \left(m_{\zeta}^{2}\right)}{6}\right.
\end{aligned}
$$

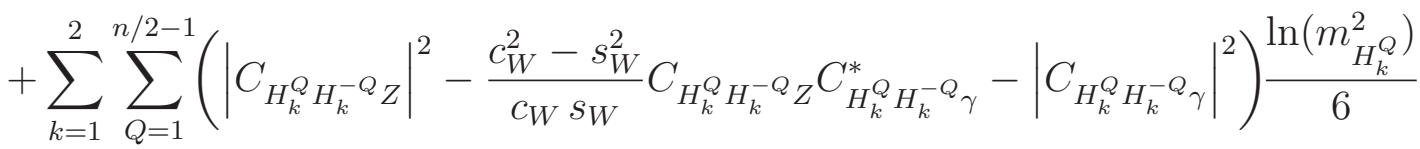

$$
\begin{aligned}
& \left.+\left|C_{I R Z}\right|^{2} f_{1}\left(m_{I}, m_{R}\right)+2 \sum_{Q=1}^{n / 2-1}\left|C_{H_{1}^{Q} H_{2}^{-Q} Z}\right|^{2} f_{1}\left(m_{H_{1}^{Q}}, m_{H_{2}^{Q}}\right)\right]
\end{aligned}
$$

Similarly, the $T$ parameter originally defined in Eq. (3.32) becomes

$$
\begin{aligned}
T=\frac{1}{4 \pi m_{Z}^{2}}[ & \sum_{r} S_{r}\left(\frac{C_{r r^{*} W^{+} W^{-}}}{c_{W}}-C_{r r^{*} Z Z}\right) f_{2}\left(m_{r}, m_{r}\right) \\
& \left.\quad-2 \sum_{s, t} \frac{\left|C_{s t W^{+}}\right|^{2}}{c_{W}} f_{2}\left(m_{s}, m_{t}\right)+2 \sum_{i, j}\left|C_{i j Z}\right|^{2} f_{2}\left(m_{i}, m_{j}\right)\right],
\end{aligned}
$$

where the couplings $C_{r r^{*} X Y}$ involving scalars $r r^{*}$ and vector bosons $X, Y$ are defined with an overall factor of $e^{2}$ removed (see Appendix A.2). The sum over states $r$ runs over $r=\left\{\zeta^{0, r}, \zeta^{0, i}, H_{k}^{Q}, \zeta^{n / 2}\right\}=\left\{I, R, H_{k}^{Q}, \zeta\right\}$, with $Q>0$ and $k=1,2$. For these couplings, $S_{r}$ is a symmetry factor given by $S_{r}=1 / 2$ for $r=\zeta^{0, r}$ or $\zeta^{0, i}$ and $S_{r}=1$ otherwise. The sums over states $s$ and $t$ run over $s t=\left\{\zeta^{0, r} H_{k}^{-}, \zeta^{0, i} H_{k}^{-}, H_{k}^{+Q} H_{l}^{-Q-1}, \zeta^{n / 2} H_{k}^{-n / 2-1}\right\}=\left\{R H_{k}^{-}, I H_{k}^{-}, H_{k}^{Q} H_{l}^{-Q-1}, \zeta H_{k}\right\}$. The sums over states $i j$ run over the same set of states given below Eq. (6.44). The 
function $f_{2}\left(m_{1}, m_{2}\right)$ has dimensions of mass-squared and is defined by Eq. (3.37).

Explicitly, this becomes

$$
\begin{aligned}
T= & \frac{1}{4 \pi m_{Z}^{2}}\left[-\frac{2}{c_{W}} \sum_{k=1}^{2}\left(\left|C_{\zeta H_{k} W}\right|^{2} f_{2}\left(m_{\zeta}, m_{H_{k}^{n / 2+1}}\right)+\left|C_{I H_{k}^{-} W}\right|^{2} f_{2}\left(m_{I}, m_{H_{k}^{+}}\right)\right.\right. \\
& \left.+\left|C_{R H_{k}^{-} W}\right|^{2} f_{2}\left(m_{R}, m_{H_{k}^{+}}\right)\right)+\frac{C_{\zeta \zeta W W}}{c_{W}} m_{\zeta}^{2} \ln \left(m_{\zeta}^{2}\right)+2\left|C_{R I Z}\right|^{2} f_{2}\left(m_{R}, m_{I}\right) \\
& +\frac{1}{2}\left(\frac{C_{R R W W}}{c_{W}}-C_{R R Z Z}\right) m_{R}^{2} \ln \left(m_{\zeta}^{2}\right)+\frac{1}{2}\left(\frac{C_{I I W W}}{c_{W}}-C_{I I Z Z}\right) m_{I}^{2} \ln \left(m_{I}^{2}\right) \\
& -\frac{2}{c_{W}} \sum_{Q=1}^{n / 2-2} \sum_{k, l=1}^{2}\left|C_{H_{k}^{Q} H_{l}^{-Q-1} W}\right|^{2} f_{2}\left(m_{H_{k}^{Q}}, m_{H_{l}^{Q+1}}\right)+4 \sum_{Q=1}^{n / 2-1}\left|C_{H_{1}^{Q} H_{2}^{-Q} Z}\right|^{2} f_{2}\left(m_{\left.H_{1}^{Q}, m_{H_{2}^{Q}}\right)}\right. \\
& \left.+\sum_{Q / 2-1}^{2} \sum_{k=1}^{2}\left[\frac{C_{H_{k}^{Q} H_{k}^{-Q} W W}}{c_{W}}-C_{H_{k}^{Q} H_{k}^{-Q} Z Z}+2\left|C_{H_{k}^{Q} H_{k}^{-Q} Z}\right|^{2}\right] m_{H_{k}^{Q}}^{2} \ln \left(m_{H_{k}^{Q}}^{2}\right)\right] . \quad(6.47)
\end{aligned}
$$

For the $U$ parameter we find,

$$
\begin{aligned}
U=\frac{2 s_{W}^{2}}{\pi}\left[\sum_{s, t}\left|C_{s t W^{+}}\right|^{2} f_{1}\left(m_{s}, m_{t}\right)\right. & \\
& \left.\quad-\sum_{i, j}\left(c_{W}^{2}\left|C_{i j Z}\right|^{2}+2 s_{W} c_{W} C_{i j Z} C_{i j \gamma}^{*}+s_{W}^{2}\left|C_{i j \gamma}\right|^{2}\right) f_{1}\left(m_{i}, m_{j}\right)\right],
\end{aligned}
$$

where the sums over states $i j$ and st run over the same sets of states given below Eqs. (6.44) and (6.46). Explicitly, this becomes

$$
\begin{aligned}
U= & \frac{2 s_{W}^{2}}{\pi}\left[\sum _ { k = 1 } ^ { 2 } \left(\left|C_{\zeta H_{k} W}\right|^{2} f_{1}\left(m_{\zeta}, m_{H_{k}^{n / 2+1}}\right)+\left|C_{I H_{k}^{-} W}\right|^{2} f_{1}\left(m_{I}, m_{H_{k}^{+}}\right)\right.\right. \\
& \left.+\left|C_{R H_{k}^{-} W}\right|^{2} f_{1}\left(m_{R}, m_{H_{k}^{+}}\right)\right)+\sum_{Q=1}^{n / 2-2} \sum_{k, l=1}^{2}\left|C_{H_{k}^{Q} H_{l}^{-Q-1} W}\right|^{2} f_{1}\left(m_{H_{k}^{Q}}, m_{H_{l}^{Q+1}}\right) \\
& -2 c_{W}^{2} \sum_{Q=1}^{n / 2-1}\left|C_{H_{1}^{Q} H_{2}^{-Q} Z}\right|^{2} f_{1}\left(m_{H_{1}^{Q}}, m_{H_{2}^{Q}}\right)-\sum_{Q=1}^{n / 2-1} \sum_{k=1}^{2}\left(c_{W}^{2}\left|C_{H_{k}^{Q} H_{k}^{-Q} Z}\right|^{2}+s_{W}^{2}\left|C_{H_{k}^{Q} H_{k}^{-Q} \gamma}\right|^{2}\right. \\
& \left.+2 s_{W} c_{W} C_{H_{k}^{Q} H_{k}^{-Q} Z} C_{H_{k}^{*} H_{k}^{-Q} \gamma}\right) \frac{\ln \left(m_{H_{k}^{Q}}^{2}\right)}{6}-c_{W}^{2}\left|C_{R I Z}\right|^{2} f_{1}\left(m_{I}, m_{R}\right) \\
& \left.-\left(c_{W}^{2}\left|C_{\zeta \zeta Z}\right|^{2}+s_{W}^{2}\left|C_{\zeta \zeta \gamma}\right|^{2}+2 s_{W} c_{W} C_{\zeta \zeta Z} C_{\zeta \zeta \gamma}^{*}\right) \frac{\ln \left(m_{\zeta}^{2}\right)}{6}\right]
\end{aligned}
$$



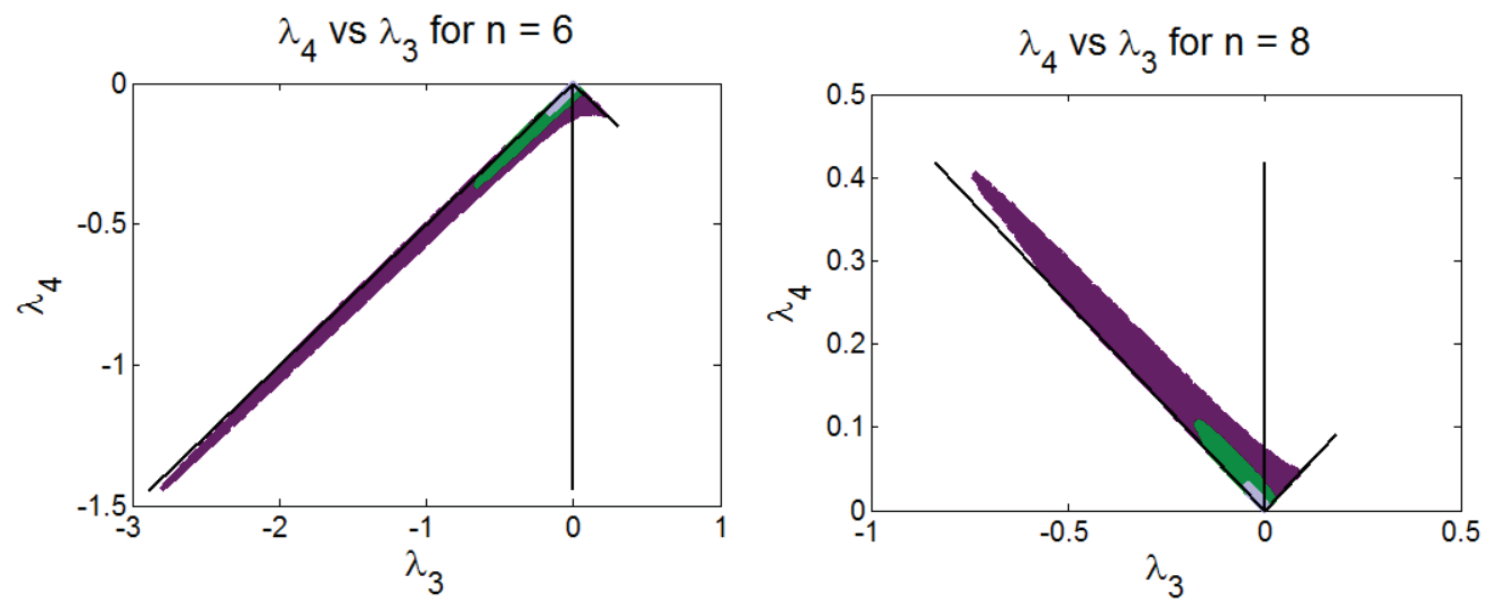

Figure 6.2: Values of $\lambda_{3}$ and $\lambda_{4}$ allowed at $95 \%$ confidence level by the oblique parameters, under the requirement that $\left|\lambda_{3}\right| \leq 2\left|\lambda_{4}\right|$, for $m_{\zeta^{0, r}}=80,150$, and $300 \mathrm{GeV}$ (light blue, medium green, and dark purple, respectively). Left: $n=6$ model. Right: $n=8$ model.

We have checked that Eqs. (6.44-6.49) reduce to the U(1)-preserving limit of Eqs. (5.21-5.23) when $\lambda_{4} \rightarrow 0$.

The contributions of the large multiplet to $S, T$ and $U$ depend only on the mass spectrum and mass-eigenstate compositions. Thus the oblique parameters constrain only $\lambda_{3}, \lambda_{4}$, and the overall mass scale, which can be parametrized by $m_{\zeta^{0, r}}$. We use the current experimental values for $S, T$ and $U$ (given by Eq. (3.52)) to constrain the $\left(\lambda_{3}, \lambda_{4}\right)$ plane for a given $m_{\zeta^{0, r}}$ using the three-observable $\chi^{2}$ variable defined in Eq. (3.50). As in the $U(1)$ case, here we use the number of free parameters (3) as the degrees of freedom and take $\chi_{0.05}^{2}=7.18$.

The ranges of $\lambda_{3}$ and $\lambda_{4}$ allowed by this constraint are shown in Fig. 6.2 for sample values $m_{\zeta^{0, r}}=80,150$, and $300 \mathrm{GeV}$; here we have also imposed the constraint $\left|\lambda_{3}\right| \leq 2\left|\lambda_{4}\right|$ (indicated by the diagonal lines), as required for $\zeta^{0, r}$ to be the lightest state. The allowed parameter space is tightly constrained to lie near the $\left|\lambda_{3}\right|=2\left|\lambda_{4}\right|$ line. This is driven by the contributions of the large multiplet to the 

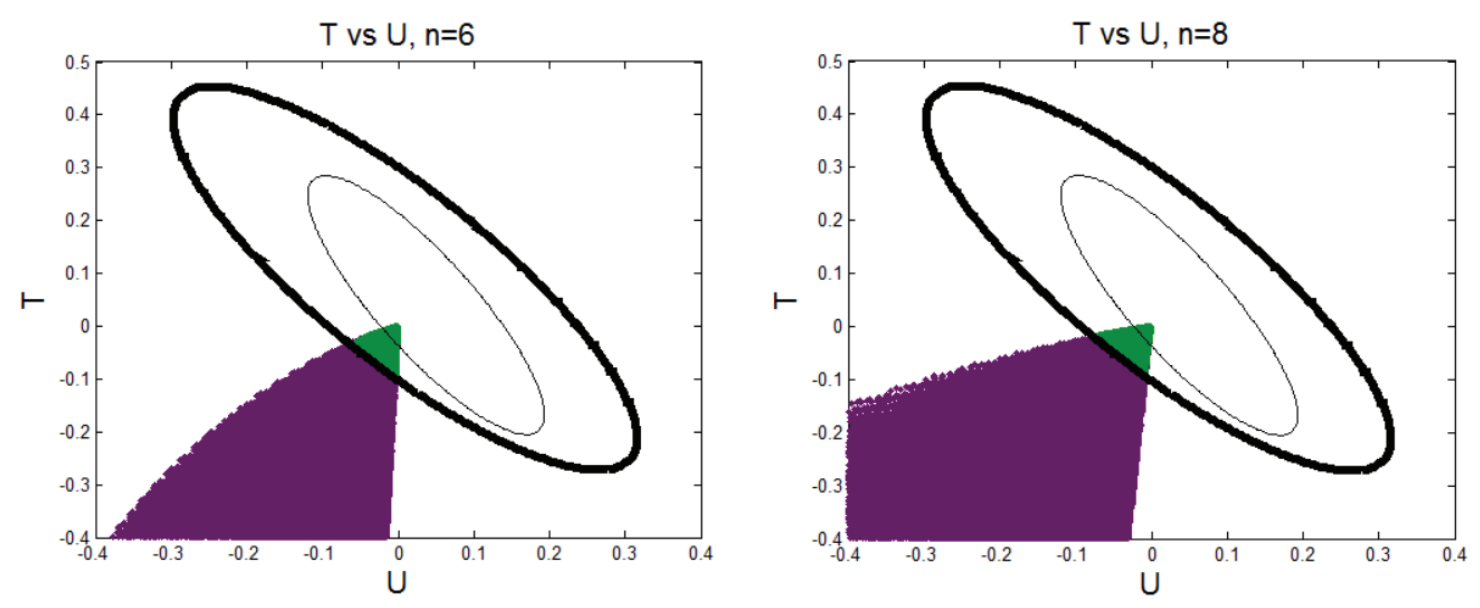

Figure 6.3: Unitarity-allowed $U$ and $T$ values for $m_{\zeta^{0, r}}=80-500 \mathrm{GeV}$ (shaded regions) and the projection of the $S, T, U 95 \%$ confidence level constraint ellipsoid (thick black ellipse). Points allowed by the $S, T, U$ constraint are shown in green (lighter shaded region). The thin black ellipse indicates the slice through the three-dimensional constraint ellipsoid at $S=0$. Left: $n=6$ model. Right: $n=8$ model.

$T$ and $U$ parameters, which rapidly become large away from this line. This feature leads to the clustering of the mass eigenstates into two groups as discussed in Sec. 6.1. Furthermore, unless both $\lambda_{3}$ and $\lambda_{4}$ are very small, the oblique parameters require $\lambda_{3}<0$. This results in the state with the largest electric charge $\left(\zeta^{+3}\right.$ for $n=6$ and $\zeta^{+4}$ for $n=8$ ) being clustered with the heavier group of mass eigenstates. The length of the allowed region along the $\left|\lambda_{3}\right| \simeq 2\left|\lambda_{4}\right|$ line is limited mainly by the $S$ parameter constraint.

To better understand the source of these constraints, we plot the projections of the $S, T, U 95 \%$ confidence level constraint ellipsoid along with the region populated by the models in Figs. 6.3, 6.4, and 6.5. The $T$ and $U$ measurements severely constrain the parameter space, as can be seen in Fig. 6.3. This in turn tightens the allowed excursion of the $S$ parameter, as shown in Figs. 6.4 and 6.5. In particular, because of the correlations among the measured values of $S, T$, and $U$, positive values of $S$ 

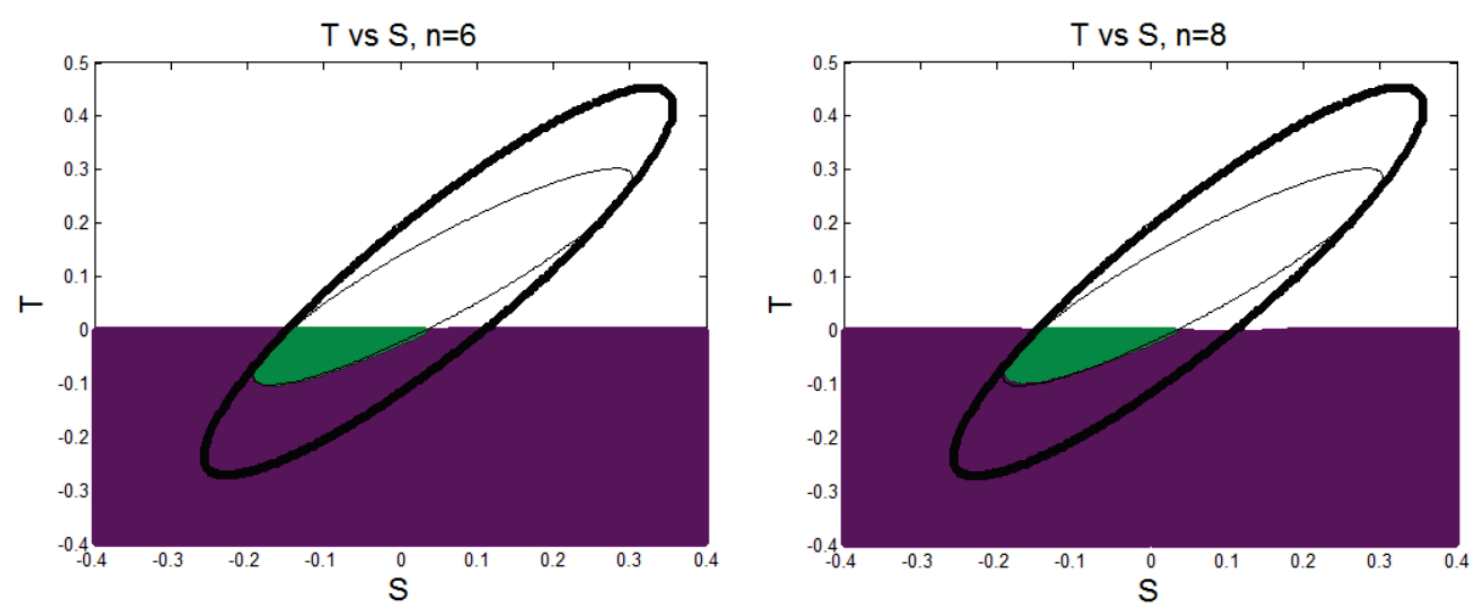

Figure 6.4: Unitarity-allowed $S$ and $T$ values for $m_{\zeta^{0, r}}=80-500 \mathrm{GeV}$ (shaded regions) and the projection of the $S, T, U 95 \%$ confidence level constraint ellipsoid (thick black ellipse). Points allowed by the $S, T, U$ constraint are shown in green (lighter shaded region). The thin black ellipse indicates the slice through the three-dimensional constraint ellipsoid at $U=0$. Left: $n=6$ model. Right: $n=8$ model.

are severely constrained. The sign of $S$ is the same as the sign of $\lambda_{3}$ in our models. This constraint leads to the preference for negative values of $\lambda_{3}$.

The oblique parameters tightly constrain the mass splittings among the lightest states of the large multiplet. The first mass splitting between $\zeta^{0, r}$ and $H_{1}^{+}$must be quite small: for $m_{\zeta^{0, r}} \leq 500 \mathrm{GeV}$, this splitting $\Delta m \equiv m_{H_{1}^{+}}-m_{\zeta^{0, r}}$ is less than 1.5 GeV in the $n=6$ model and less than $0.7 \mathrm{GeV}$ in the $n=8$ model (see Fig. 6.6).

The splitting between the heavier group of states and the lighter group of states, on the other hand, is much less tightly constrained. The allowed mass splitting between the lightest state $\zeta^{0, r}$ and the heavier singly-charged state $H_{2}^{+}, \Delta M \equiv m_{H_{2}^{+}}$ $m_{\zeta^{0, r}}$, is shown in Fig. 6.7 as a function of $m_{\zeta^{0, r}}$. For the $n=6$ model, this splitting can be as large as $100 \mathrm{GeV}$ for $m_{\zeta^{0, r}}=100 \mathrm{GeV}$, growing to more than $450 \mathrm{GeV}$ for $m_{\zeta^{0, r}}=500 \mathrm{GeV}$. For the $n=8$ model the maximum allowed mass splitting is about half as large, ranging between about $50 \mathrm{GeV}$ and $200 \mathrm{GeV}$ for $m_{\zeta^{0, r}}$ between 100 and 

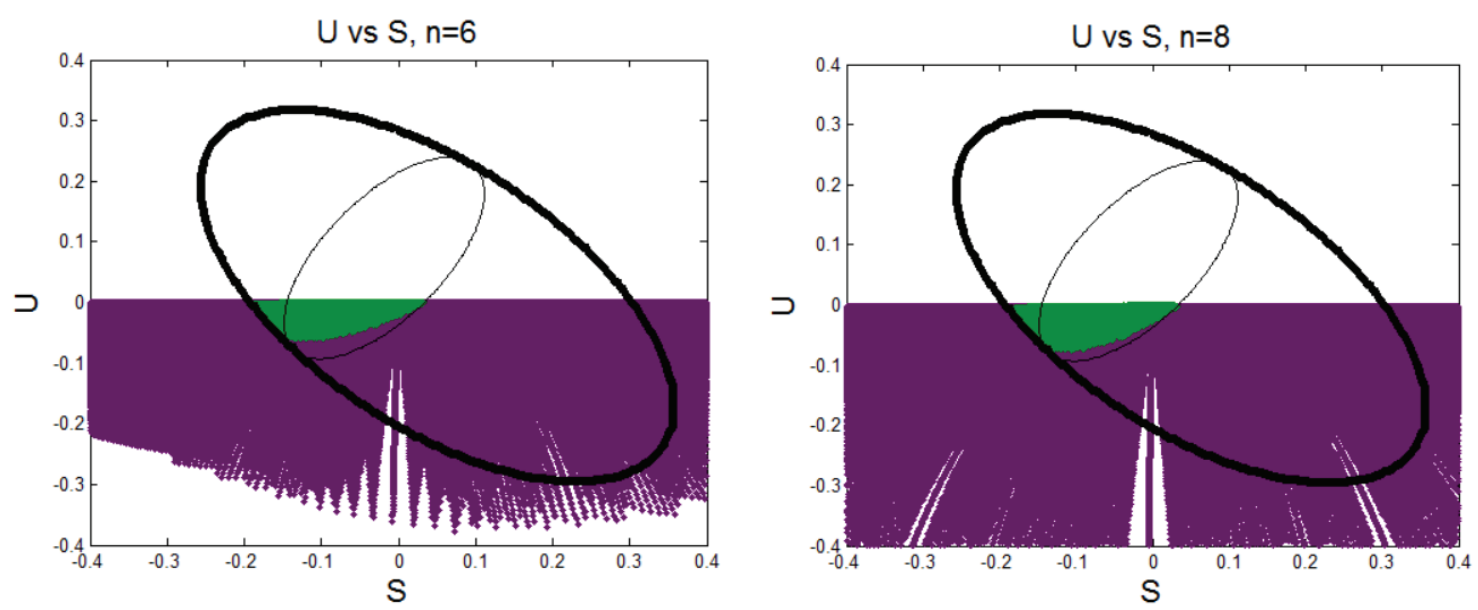

Figure 6.5: Unitarity-allowed $S$ and $U$ values for $m_{\zeta^{0, r}}=80-500 \mathrm{GeV}$ (shaded regions) and the projection of the $S, T, U 95 \%$ confidence level constraint ellipsoid (thick black ellipse). Points allowed by the $S, T, U$ constraint are shown in green (lighter shaded region). The thin black ellipse indicates the slice through the three-dimensional constraint ellipsoid at $T=0$. Scatter in the plot is due to the numerical scan. Left: $n=6$ model. Right: $n=8$ model.

$500 \mathrm{GeV}$. The linear growth of the maximum allowed mass splitting shown in Fig. 6.7 is eventually cut off by the unitarity constraints on $\lambda_{3}$ and $\lambda_{4}$ for large enough $m_{\zeta^{0, r}}$.

As briefly discussed at the end of Sec. 5.3.1, small shifts in Figs. 6.2-6.7 when compared with the original publication, Ref. [3] are due to an update in the experimental values for the oblique parameters (Ref. [89] vs Ref. [124]). This update does not affect our conclusions.

\subsubsection{Constraints from $h \rightarrow \gamma \gamma$ decay}

To constrain the parameter $\lambda_{2}$, we consider the one-loop contributions of the charged scalars to the Higgs decays $h \rightarrow \gamma \gamma^{3}$ and $h \rightarrow Z \gamma$. The decay partial width for

\footnotetext{
${ }^{3}$ Contributions to $h \rightarrow \gamma \gamma$ from multiply-charged scalars in the loop have also been considered in Refs. $[16,26]$.
} 

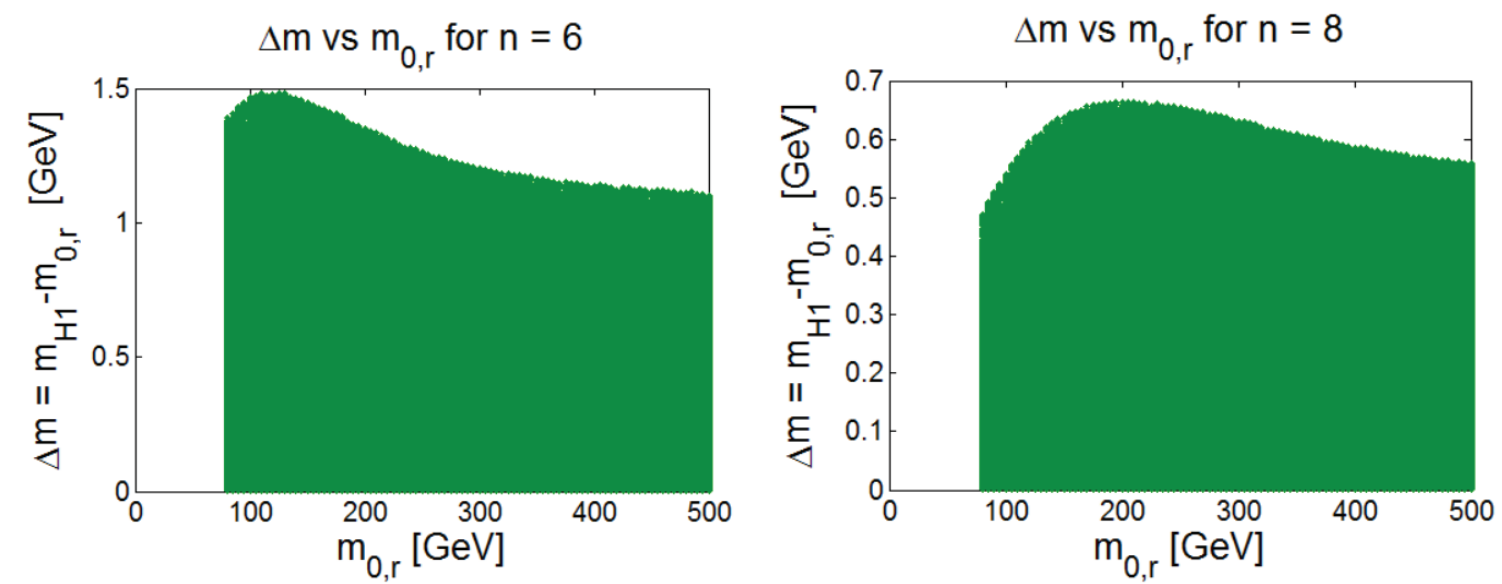

Figure 6.6: Allowed range for the first mass splitting $\Delta m \equiv m_{H_{1}^{+}}-m_{\zeta^{0, r}}$ as a function of $m_{\zeta^{0, r}}$. Left: $n=6$ model. Right: $n=8$ model.

$h \rightarrow \gamma \gamma$ is defined by Eq. (3.57). In the $Z_{2}$ models the scalars $s=\left\{H_{1}^{Q}, H_{2}^{Q}, \zeta^{n / 2}\right\}$ may propagate in the loop, and the scalar contribution becomes

$$
\begin{aligned}
A_{S}^{h \gamma \gamma}= & \frac{n^{2}}{m_{h}^{2}} C_{h \zeta^{n / 2} \zeta^{n / 2 *}} \frac{v}{2}\left[1-\frac{4 m_{\zeta^{n / 2}}^{2}}{m_{h}^{2}} f\left(\frac{4 m_{\zeta^{n / 2}}^{2}}{m_{h}^{2}}\right)\right] \\
& +\sum_{i=1}^{2} \sum_{Q=1}^{n-1} \frac{2 v Q^{2} C_{h H_{i}^{Q} H_{i}^{-Q}}}{m_{h}^{2}}\left[1-\frac{4 m_{H_{i}^{Q}}^{2}}{m_{h}^{2}} f\left(\frac{4 m_{H_{i}^{Q}}^{2}}{m_{h}^{2}}\right)\right],
\end{aligned}
$$

where $f(\tau)$ is defined by Eq. (3.63), the masses are defined in Eqs. (6.11) and (6.17), and the couplings are defined in Appendix A.2. Note that despite the mixing between the multiplet states, the loops contributing to $h \rightarrow \gamma \gamma$ contain only one species of scalar as the photon couplings are always diagonal. The $h \rightarrow \gamma \gamma$ decay width is then defined by Eq. (3.57) as before, where contributions from the $W$ boson and the top quark are respectively given by Eq. (3.60) and (3.65) and $\kappa_{f}=\kappa_{W}=1$.

Eq. (6.50) depends on $\lambda_{2,3,4}$ and $m_{\zeta^{0, r}}$, and may be constrained by applying the experimental bounds from ATLAS and CMS (summarized in Table 3.1). However, this bound does not substantially constrain $\lambda_{3}, \lambda_{4}$ and $m_{\zeta^{0, r}}$ beyond the bounds from theoretical and oblique constraints. As a result, the $h \rightarrow \gamma \gamma$ bound primarily 

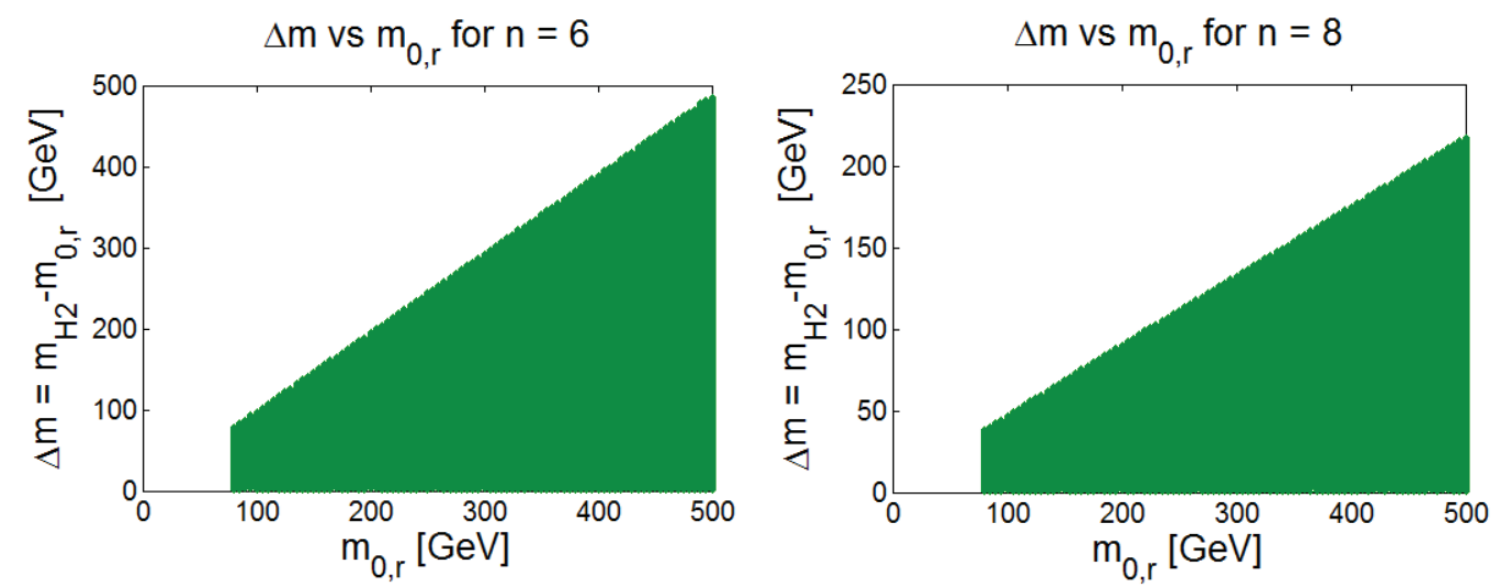

Figure 6.7: Allowed range for one of the larger mass splittings, $\Delta M \equiv m_{H_{2}^{+}}-m_{\zeta^{0, r}}$, as a function of $m_{\zeta^{0, r}}$. Left: $n=6$ model. Right: $n=8$ model.

constrains $\lambda_{2}$. We find the allowed range of $\lambda_{2}$ as a function of $m_{\zeta^{0, r}}$ by scanning over the values of $\lambda_{3}$ and $\lambda_{4}$ allowed by the oblique parameter constraints and perturbative unitarity. As described in Sec. 3.2.4, we accept points for which $R_{\gamma \gamma}$ falls within the measured $2 \sigma$ range for $\mu_{\gamma \gamma}$. Results are shown in Fig. 6.8.

Note that the constraint from perturbative unitarity, $\left|\lambda_{2}\right| \leq 6.59$ (3.10) for $n=6$ (8), is visible in Fig. 6.8. The upper branch of allowed $\lambda_{2}$ values corresponds to a sign flip of the total $h \rightarrow \gamma \gamma$ amplitude relative to the SM prediction. This is separated from the rest of the points due to the lower bound on $\mu_{\gamma \gamma}$.

One may note that the $\lambda_{2}-m_{0, r}$ parameter space displayed in Fig. 6.8 is more tightly constrained than that of the corresponding figure originally published in Ref. [3]. The experimental measurements of $h \rightarrow \gamma \gamma$ have substantially improved since this calculation was originally performed in 2013. At that time there was a substantial difference between the respective measurements from ATLAS and CMS; the ATLAS central value was substantially higher (1.65 in comparison to the current 1.17) while the CMS central value was substantially lower (0.78 in comparison with 

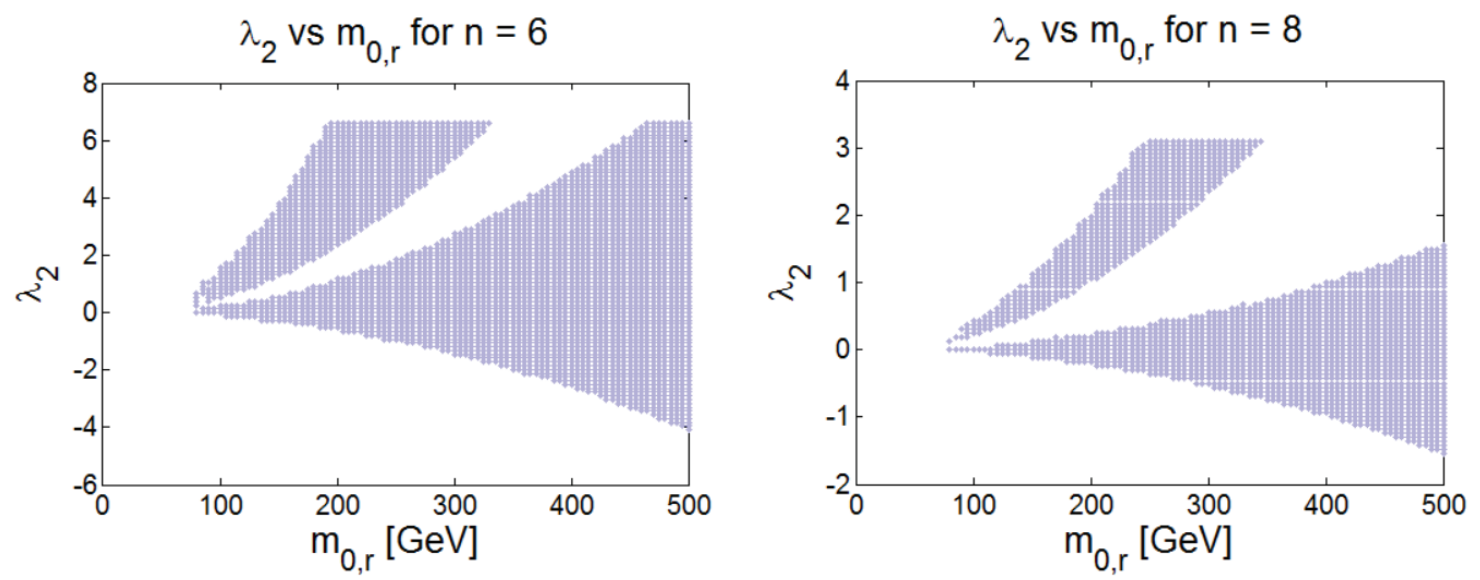

Figure 6.8: Allowed ranges of $\lambda_{2}$ as a function of $m_{\zeta^{0, r}}$ after imposing the LHC constraint on the Higgs decay to two photons, together with the oblique parameter constraints and perturbative unitarity. Left: $n=6$ model. Right: $n=8$ model.

the current 1.14). To be conservative, in Ref. [3] we accepted points within $2 \sigma$ of either measurement. However, the new data and higher statistics accumulated over the past two years have greatly improved the correspondence between the ATLAS and CMS measurements of the $h \rightarrow \gamma \gamma$ signal, resulting in a much smaller acceptance window. This improvement in the $h \rightarrow \gamma \gamma$ constraint has reduced the allowed parameter space along each curved edge of the regions depicted in Fig. 6.8, resulting in the appearance of a larger separation between the upper and lower branches.

\subsubsection{Predictions for $h \rightarrow Z \gamma$}

The charged scalars in our models also contribute to the loop-induced decay $h \rightarrow Z \gamma$. The decay partial width for this process is defined in Eq. (3.68). In the $Z_{2}$ model, the contributions from fermions and $W$ bosons are respectively given by Eqs. (3.71) and (3.73) with the usual SM values and $\kappa_{f}=\kappa_{W}=1$. The contribution from scalars is given by Eq. (3.70), where the couplings $C_{h s s}$ and $C_{s s Z}$ are defined in Appendix A.2. 

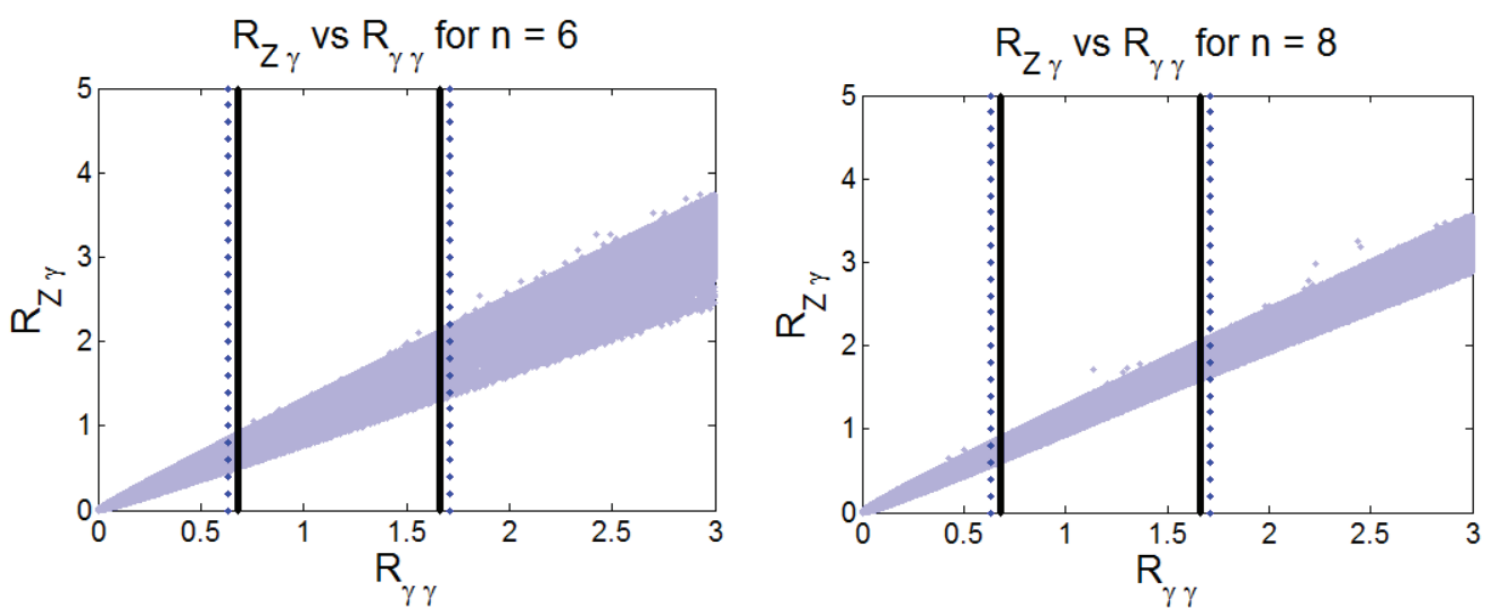

Figure 6.9: Allowed range of the $h \rightarrow Z \gamma$ partial width scaling factor $R_{Z \gamma}$ plotted against the $h \rightarrow \gamma \gamma$ partial width scaling factor $R_{\gamma \gamma}$ [see Eq. (3.56)]. Light blue points represent a scan over the parameter space allowed by perturbative unitarity and the oblique parameters $S, T$, and $U$, for $m_{\zeta^{0, r}}$ in the range $80-500 \mathrm{GeV}$. Vertical lines indicate the $2 \sigma$ bound of the experimental $h \rightarrow \gamma \gamma$ measurement from ATLAS [126] (dotted blue) or CMS [127] (solid black). Left: $n=6$ model. Right: $n=8$ model.

As in the previous section the sum over $s$ runs over all charged scalars in the model, $s=\left\{H_{1}^{Q}, H_{2}^{Q}, \zeta^{n / 2}\right\}$. Note again that each scalar loop contribution contains only one species of scalar, as the couplings of scalars to both photons and the Higgs boson are diagonal in this model.

The LHC experiments currently constrain the rate for $h \rightarrow Z \gamma$ to be less than about 10 times the SM prediction (see Table 3.1). This does not further constrain the parameter space of our models once the constraints from perturbative unitarity, oblique parameters, and $h \rightarrow \gamma \gamma$ have been applied. Indeed, we find $R_{Z \gamma}$ to be strongly correlated with $R_{\gamma \gamma}$, as shown in Fig. 6.9. Imposing all experimental constraints previously discussed and scanning $m_{\zeta^{0, r}}$ in the range $80-500 \mathrm{GeV}$, we find that the ratio $R_{Z \gamma} / R_{\gamma \gamma}$ lies between approximately 0.7 and 1.3 for the $n=6$ model, and between approximately 0.9 and 1.2 for the $n=8$ model but with some points higher than the quoted range, as can be seen in Fig. 6.9. 


\subsubsection{Dark matter constraints}

As in the $U(1)$ model, in the $Z_{2}$ model the lightest neutral scalar $\zeta^{0, r}$ is a dark matter candidate. Under the assumption of a standard thermal history of the universe, as discussed in Sec. 3.2.6, the fraction of the total dark matter that is made up by $\zeta^{0, r}$ can be related to its annihilation cross section according to Eq. (3.109), and bounded from above by direct detection constraints Eq. (3.110).

In the $U(1)$ model case, the dark matter candidate was a complex scalar that annihilated with its anti-particle via both $Z$ and Higgs exchange. These models were excluded by the dark matter direct detection bounds due to the large contributions to the nucleon scattering cross section from $Z$ exchange. In the $Z_{2}$ models, the situation is quite different as the dark matter candidate is a real scalar. It couples to the $Z$ boson only with the second heavier neutral scalar $\zeta^{0, i}$ through a $Z \zeta^{0, r} \zeta^{0, i}$ vertex. As a result, the $Z$ exchange scattering process that excluded the $U(1)$ models is kinematically inaccessible in the $Z_{2}$ models, allowing them to remain viable.

In a high-mass region where mass splittings are small, it could be possible for the $\zeta^{0, r}$ to up-scatter to a $\zeta^{0, i}$ through $Z$ exchange, which in turn could result in a nucleon scattering cross section large enough to violate the direct-detection limits (as in the $U(1)$ case). However, a calculation of the accessible mass splitting in terms of the ambient $\zeta^{0, r}$ velocity [3] indicates that the mass splittings between the electricallycharged scalars and $\zeta^{0, r}$ would be less than the mass of an electron in this scenario. This possibility is excluded by the absence of heavy charged relics, as a mass splitting of this size would require that the electrically-charged scalars be stable. 

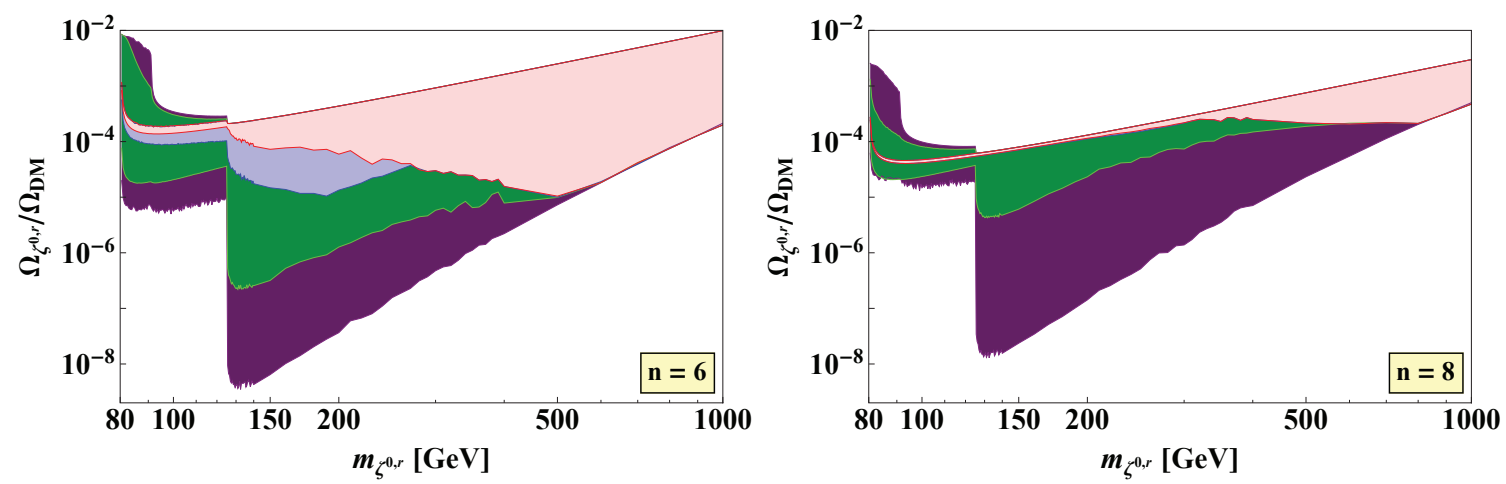

Figure 6.10: The fraction $\Omega_{\zeta^{0, r}} / \Omega_{\mathrm{DM}}$ of the total dark matter density that can be made up of $\zeta^{0, r}$ as a function of $m_{\zeta^{0, r}}$. The colored regions show the accessible range of $\Omega_{\zeta^{0, r}} / \Omega_{\mathrm{DM}}$ obtained from a numerical scan over parameter space after successively applying constraints from perturbative unitarity (dark purple), the oblique parameters (medium green), Higgs decays to two photons (light blue), and $M^{2}>0$ to avoid alternative vacua (very light pink). Left: $n=6$ model. Right: $n=8$ model.

\section{Thermal relic density}

The $\zeta^{0, r}$ annihilates with itself to $W^{+} W^{-}, Z Z, h h$, and $f \bar{f}$ two-body final states through gauge boson and Higgs exchange. These annihilation processes were computed by collaborators Heather Logan and Terry Pilkington in Ref. [3] in the zerovelocity limit, neglecting co-annihilations with other scalars from the $Z_{2}$-odd multiplet. The corresponding relic density to this annihilation cross section is plotted in Fig. 6.10. After scanning over $\lambda_{2}, \lambda_{3}$, and $\lambda_{4}$, the constraints from perturbative unitarity (dark purple regions), the oblique parameters (medium green regions), Higgs decays to two photons (light blue regions), and $M^{2}>0$ to avoid alternative vacua (very light pink regions) have been applied in succession.

One will note that at $m_{\zeta^{0, r}}>125 \mathrm{GeV}$ the relic density is suddenly permitted to be much smaller, as this is the kinematic threshold for the $\zeta^{0, r} \zeta^{0, r} \rightarrow h h$ process. However, the imposition of the constraints from oblique parameters (medium green) and $h \rightarrow \gamma \gamma$ (light blue) severely reduce this drop in relic density, as they limit the 
allowed strength $\Lambda_{n}$ of the $h \zeta^{0, r} \zeta^{0, r}$ and $h h \zeta^{0, r} \zeta^{0, r}$ couplings. For $\zeta^{0, r}$ masses above about $500 \mathrm{GeV}$ in the $n=6$ model $(800 \mathrm{GeV}$ in the $n=8$ model $)$ the constraints on $\lambda_{2}, \lambda_{3}$, and $\lambda_{4}$ come only from perturbative unitarity.

As seen in Fig. 6.10, the thermal relic abundance of $\zeta^{0, r}$ can account for at most $1 \%$ of the dark matter for masses $m_{\zeta^{0, r}}$ below $1 \mathrm{TeV}$. In this mass range, both the $n=6$ and $n=8$ models are consistent with the observed dark matter relic abundance under the assumption that most of the dark matter is made up of some other candidate particle. An extension of these results to higher masses [3] suggests that $\zeta^{0, r}$ may be able to account for all the dark matter for masses of 10-33 TeV for the $n=6$ model, or $18-30 \mathrm{TeV}$ for the $n=8$ model.

\section{Dark matter direct detection}

In the $Z_{2}$ model, the scattering of $\zeta^{0, r}$ off a nucleon $N=p, n$ proceeds only through Higgs exchange. The resulting spin-independent per-nucleon cross section required for the constraint of Eq. (3.110) is given by

$$
\sigma_{\mathrm{SI}}^{\zeta}=\frac{\left(f_{N}^{h}\right)^{2} \Lambda_{n}^{2} v^{2}}{4 \pi M_{h}^{4}} \frac{m_{N}^{2}}{\left(m_{N}+m_{\zeta^{0, r}}\right)^{2}},
$$

where $\Lambda_{n}$ is defined in Eqs. (6.26) and (6.36) for $n=6$ and $n=8$ respectively, and the Higgs-nucleon Yukawa couplings are given by [130]

$$
\begin{aligned}
f_{p}^{h} & =\frac{m_{p}}{v}(0.350 \pm 0.048), \\
f_{n}^{h} & =\frac{m_{n}}{v}(0.353 \pm 0.049) .
\end{aligned}
$$

Because the relic density of $\zeta^{0, r}$ is only a fraction of the total dark matter density, the direct-detection scattering cross section $\sigma_{\mathrm{SI}}^{\mathrm{exp}}$ quoted by experiments does not correspond directly to $\sigma_{\mathrm{SI}}^{\zeta}$, but rather to $\sigma_{\mathrm{SI}}^{\zeta}$ scaled by the fraction of the total dark 

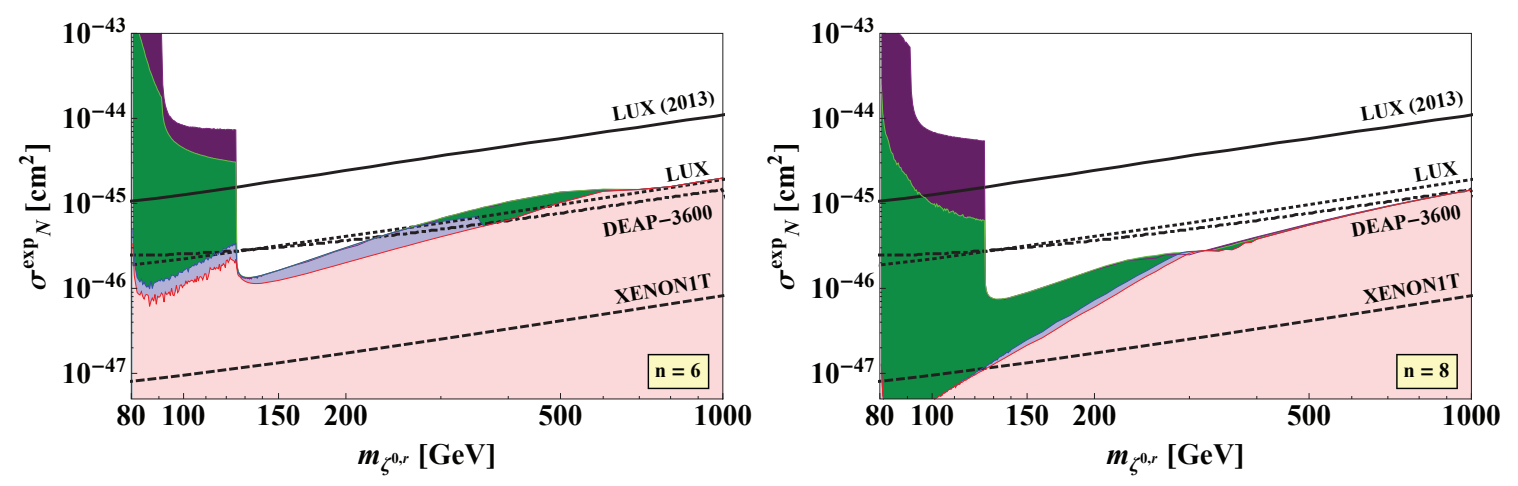

Figure 6.11: Predictions for the experimentally-defined direct detection cross section $\sigma_{\mathrm{SI}}^{\exp }$ [see Eq. (6.53)] as a function of $m_{\zeta^{0, r}}$. The colored regions show the accessible range of $\sigma_{\mathrm{SI}}^{\exp }$ after successively applying constraints from perturbative unitarity (dark purple), the oblique parameters (medium green), Higgs decays to two photons (light blue), and $M^{2}>0$ to avoid alternative vacua (very light pink). Also shown are the current experimental limit from the LUX experiment [120] (solid black curve), as well as projections for the ultimate sensitivities of the LUX, DEAP-3600, and XENON1T experiments [116] (dashed curves). Left: $n=6$ model. Right: $n=8$ model.

matter density that is made up by $\zeta^{0, r}$ :

$$
\sigma_{\mathrm{SI}}^{\exp }=\frac{\Omega_{\zeta^{0, r}}}{\Omega_{\mathrm{DM}}} \sigma_{\mathrm{SI}}^{\zeta}
$$

where $\frac{\Omega_{\zeta^{0}, r}}{\Omega_{\mathrm{DM}}}$ is the relic density ratio calculated in the previous section.

Fig. 6.11 shows the predicted range of density-scaled experimental direct detection cross sections $\sigma_{\mathrm{SI}}^{\exp }$ as a function of $m_{\zeta^{0, r}}$ for the models with $n=6$ and 8. As before, we scan over $\lambda_{2}, \lambda_{3}$, and $\lambda_{4}$, applying in succession the constraints from perturbative unitarity (dark purple regions), the oblique parameters $S, T$, and $U$ (medium green regions), Higgs decays to two photons (light blue regions), and $M^{2}>0$ to avoid alternative vacua (very light pink regions). Because the scattering cross section mediated by Higgs exchange is proportional to $\Lambda_{n}^{2}, \sigma_{\mathrm{SI}}^{\exp }$ can be made arbitrarily small by tuning $\lambda_{2}, \lambda_{3}$ and $\lambda_{4}$ so that $\Lambda_{n} \ll 1{ }^{4}$

\footnotetext{
${ }^{4}$ In the limit that $\Lambda_{n} \rightarrow 0, \sigma_{\text {SI }}^{\zeta} \sim 10^{-(46-48)} \mathrm{cm}^{2}$ due to loop-induced scattering processes mediated by electroweak gauge bosons [131], leading to $\sigma_{\mathrm{SI}}^{\exp } \sim 10^{-(48-53)} \mathrm{cm}^{2}$.
} 
For $\zeta^{0, r}$ masses in our range of interest, at the time of calculation the strongest experimental upper limit on $\sigma_{\mathrm{SI}}^{\exp }$ was due to the LUX experiment [120], which was not sensitive enough to probe the allowed parameter space. Experimental projections for the future sensitivities of the LUX [119] and DEAP-3600 [121,122] experiments — which are currently under way - suggest that these experiments will only begin to probe the upper edge of the $n=6$ model. However, it is expected that the future XENON1T [117] and SuperCDMS [35] experiments will be sensitive enough to probe deep into the allowed model parameter space, reaching a full order of magnitude beyond the largest experimental direct-detection cross sections allowed in both the $n=6$ and $n=8$ models. Furthermore, the proposed LUX upgrade LZ is expected to reach an order of magnitude deeper in $\sigma_{\mathrm{SI}}^{\mathrm{exp}}$ than XENON1T [35] (see Fig. 3.8). 


\section{Chapter 7}

\section{The Georgi-Machacek model}

In this chapter, we consider the phenomenology of the Georgi-Machacek (GM) model. This model extends the Standard Model (SM) with two scalar electroweak triplets, one

of which is real $(\xi, Y=0)$ and one of which is complex $(\chi)$ with hypercharge $Y=2$. Both of these triplets acquire vacuum expectation values ( $v_{\xi}$ and $v_{\chi}$ respectively) which are required to be equal, $v_{\xi}=v_{\chi}$. The construction of this model — particularly the tuning of $v_{\chi}$ and $v_{\xi}$ - is carefully engineered to preserve custodial symmetry at tree-level. Although each triplet individually would violate the custodial symmetry $S U(2)_{c}$, together they preserve the relationship between the gauge boson masses and avoid violating the constraints of the $\rho$ parameter.

In comparison with Eq. (2.17), the two triplets $\chi$ and $\xi$ contribute to the gauge boson masses through their kinetic couplings as follows:

$$
\begin{gathered}
\frac{1}{2}\left|\mathcal{D}_{\mu} \xi\right|^{2} \supset \frac{v_{\xi}^{2}}{2}\left(\begin{array}{ll}
W_{\mu}^{-} & W_{\mu}^{+}
\end{array}\right)\left(\begin{array}{cc}
g^{2} & 0 \\
0 & g^{2}
\end{array}\right)\left(\begin{array}{c}
W_{\mu}^{+} \\
W_{\mu}^{-}
\end{array}\right), \\
\left|\mathcal{D}_{\mu} \chi\right|^{2} \supset \frac{v_{\chi}^{2}}{2}\left[\left(\begin{array}{ll}
W_{\mu}^{-} & W_{\mu}^{+}
\end{array}\right)\left(\begin{array}{cc}
g^{2} & 0 \\
0 & g^{2}
\end{array}\right)\left(\begin{array}{c}
W_{\mu}^{+} \\
W_{\mu}^{-}
\end{array}\right)+2\left(\begin{array}{ll}
W_{\mu}^{3} & B_{\mu}
\end{array}\right)\left(\begin{array}{cc}
g^{2} & -g g^{\prime} \\
-g g^{\prime} & g^{\prime 2}
\end{array}\right)\left(\begin{array}{c}
W_{\mu}^{3} \\
B_{\mu}
\end{array}\right)\right],
\end{gathered}
$$

The real triplet $\xi$ contributes only to the masses of $W^{ \pm}$as it has $Y=0$. The complex 
triplet $\chi$ contributes to all of the gauge boson mass matrices, but in the wrong ratio; it contributes twice as much to the $\left(W^{3}, B\right)$ matrix as it does to the $W^{ \pm}$matrix. In the presence of these contributions, the $\rho$-parameter of Eq. (3.24) has the form

$$
\rho=\frac{v_{\phi}^{2}+4 v_{\xi}^{2}+4 v_{\chi}^{2}}{v_{\phi}^{2}+8 v_{\chi}^{2}},
$$

where $v_{\phi}$ is the vacuum expectation value (vev) of the usual SM Higgs doublet. We can see from Eqs. (7.1)-(7.3) that $v_{\xi}=v_{\chi}$ is required to satisfy $\rho=1.00040 \pm 0.00024[43]$ and for the sum of the contributions to the gauge boson masses to have the same form as in Eq. (2.17) (as required by custodial symmetry). We further require that $v^{2}=v_{\phi}^{2}+8 v_{\chi}^{2}$ in order to reproduce the usual SM vev $v=246 \mathrm{GeV}$ and obtain the correct gauge boson masses $m_{W}$ and $m_{Z}$.

As the GM model preserves the custodial symmetry in its scalar potential, the scalar particles may be categorized into multiplets of $S U(2)_{c}$. The scalar spectrum comprises two custodial singlets $h$ and $H$, a triplet containing $H_{3}^{0}$ and $H_{3}^{ \pm}$, and a fiveplet constraining $H_{5}^{0}, H_{5}^{ \pm}$, and $H_{5}^{ \pm \pm}$. As we will see, the $H_{5}$ scalars are fermiophobic, i.e. they do not couple to fermions. The $H_{3}$ states, in contrast, are fermiophilic; they couple preferentially to fermions, as the $H_{3} V V$ couplings to two vector bosons are forbidden.

The GM model provides a valuable benchmark for searches for BSM physics in Higgs processes. A compelling reason for considering large scalar multiplets in Higgs models is that they allow for a larger range of phenomenology than doublet and singlet extensions. In particular, doublet and singlet models always have Higgs couplings to vector bosons that are smaller than or equal to those of the SM. For example, consider the $h W^{+} W^{-}$coupling; the current measurement of this coupling at the LHC is centered on the SM value. In the SM, the Two Higgs Doublet model 
(2HDM), and the singlet extension of the SM, this coupling is defined as follows:

$$
C_{h W W}^{\mathrm{SM}}=\frac{g^{2} v_{\mathrm{SM}}}{2}, \quad C_{h W W}^{2 \mathrm{HDM}}=\frac{g^{2} v_{\mathrm{SM}}}{2} \sin (\beta-\alpha), \quad C_{h W W}^{\text {singlet }}=\frac{g^{2} v_{\mathrm{SM}}}{2} \cos \alpha,
$$

where $\alpha$ and $\beta$ are mixing angles. Note that due to the additional factors of mixing angles the 2HDM and singlet model couplings will always be smaller than the SM coupling. Therefore half of the experimental range allowed by the experimental uncertainty on the $C_{h W W}$ measurement cannot be accessed by a $2 \mathrm{HDM}$ or a singlet extension of the SM. However, the full experimental range of this coupling could be accounted for by a larger scalar extension. If we consider the general form of the contribution to this coupling from an electroweak multiplet $\mathcal{S}$ of arbitrary isospin $T$ (corresponding to size $n=2 T+1$ ),

$$
C_{S W W}=\frac{g^{2}}{2} v_{\mathcal{S}}\left[T(T+1)-\frac{Y^{2}}{4}\right],
$$

we can see that the $C_{S W W}$ contribution may be larger than the SM $C_{h W W}$ coupling if the scalar multiplet has isospin $T \geq 1$, meaning that the multiplet size $n \geq 3$ (the multiplet must be larger than a doublet). The multiplet must also have a nonnegligible vacuum expectation value and must mix with the SM doublet state(s) in order to contribute to the observed $125 \mathrm{GeV}$ Higgs. Higgs models with large scalar multiplets therefore have the ability to enhance Higgs couplings, which in turn may account for a greater range of phenomenology.

The potential for enhanced Higgs couplings is important, as it may provide a mechanism for hiding BSM physics effects in measurements of Higgs processes. As results from the LHC are currently all in remarkably good agreement with the SM, it is important to consider mechanisms by which new physics might be concealed in these measurements. As discussed in Sec. 3.2.1, the Higgs rates that are measured at the LHC — originally defined by Eq. (3.22) — are proportional to both the Higgs 
production cross section $\left(\sigma_{h}\right)$ and the branching ratio for the relevant Higgs decay $(B R(h \rightarrow X))$. If all of the Higgs couplings are enhanced by roughly the same factor $\kappa$ in comparison to the SM, then all of the production cross sections will similarly be enhanced. This overall enhancement could compensate for a decrease in the Higgs branching ratios to SM particles due to decays to new states in the model for which no dedicated search has been performed. If the two effects balance according to

$$
\kappa^{2} \simeq \frac{1}{1-\mathrm{BR}_{\mathrm{new}}}
$$

where $\mathrm{BR}_{\text {new }}$ is the branching ratio to new particles, then the measured Higgs rates would appear SM-like, even though new physics is present. The GM model provides an interesting benchmark of a mechanism for enhanced couplings and the resulting phenomenology.

In addition, the GM model also provides benchmarks for searches for exotically charged scalars. Unlike many other popular models - MSSM, 2HDM, etc - the GM model includes a doubly charged scalar state. As such, its phenomenology can be used to guide searches for doubly charged scalars at experiments such as ATLAS and CMS. It has also been extended to incorporate the Little Higgs [37,38], SUSY [39-41], and neutrino seesaw [42] mechanisms, and can therefore provide benchmarks for these frameworks as well.

The GM mechanism was first proposed in 1987 in Ref. [30], and a scalar potential for the model was first introduced by Chanowitz and Golden in Ref. [70]. However, this original scalar potential was not the most general potential available, as it preserved an additional $Z_{2}$ symmetry in the interest of simplicity. ${ }^{1}$ In this case, the GM scalar masses are bounded to be below roughly $700 \mathrm{GeV}$ [5]. The phenomenology of the model under this additional $Z_{2}$ symmetry has been studied in

\footnotetext{
${ }^{1}$ As a result, the parameters $M_{1,2}$ introduced in the following section were required to be zero.
} 
Refs. [5,94,132-141]. Additional phenomenology independent of the scalar potential has been studied in Refs. $[142,143]$.

However, in the following work we make use of the most general scalar potential, which was first considered by Aoki and Kanemura in Ref. [5]. As we will show in Sec. 7.3, in this case there is no upper bound on the BSM masses, which allows for a decoupling limit in the model; the BSM particles can be made arbitrarily heavy, so that an SM-like theory is left at the electroweak scale. The phenomenology of this general GM model has subsequently been studied in Refs. [4,6,7,144-148]. These studies include the application of constraints from unitarity in Ref. [5] and partial constraints from the boundedness of the potential in Ref. [144]. Constraints from the oblique parameter $S$ have been considered in Refs. $[6,32,138]$ and constraints from the $Z$-pole observable $R_{b}$ have been applied in Refs. $[6,94,144]$. We both reproduce and expand upon many of these constraints in the following work.

Throughout this chapter, we outline the constraints that may be placed upon the parameter space of the GM model. In Sec. 7.2 we apply theoretical constraints from unitarity, the boundedness of the scalar potential, and the lack of alternative vacua. In Sec. 7.3 we consider the phenomenology of the model in its decoupling limit, and suggest methods of distinguishing the GM model from the similar 2HDM in this limit. In Sec. 7.4 we apply constraints from indirect experimental measurements such as the oblique parameters, $R_{b}, b \rightarrow s \gamma, B_{s}^{0}-\bar{B}_{s}^{0}$ meson mixing, and $B_{s}^{0} \rightarrow \mu^{+} \mu^{-}$. We study the effect of these constraints on the GM parameter space, placing particular emphasis on the ability of the model to enhance the couplings of the $125 \mathrm{GeV}$ Higgs. In Sec. 7.5 we review bounds from direct collider searches. Finally, in Sec. 7.6 we consider the loop-decays of the BSM scalars in the GM model. 


\subsection{Model description}

The scalar sector of the GM model consists of the usual complex electroweak doublet $\left(\phi^{+}, \phi^{0}\right)$ with hypercharge $Y=1$, a real triplet $\left(\xi^{+}, \xi^{0}, \xi^{-}\right)$with $Y=0$, and a complex triplet $\left(\chi^{++}, \chi^{+}, \chi^{0}\right)$ with $Y=2[30]$. The doublet is responsible for the fermion masses as in the SM. In order to make the global $\mathrm{SU}(2)_{L} \times \mathrm{SU}(2)_{R}$ symmetry explicit, we write the doublet in the form of a bi-doublet $\Phi$ and combine the triplets to form a bi-triplet $X$ :

$$
\Phi=\left(\begin{array}{cc}
\phi^{0 *} & \phi^{+} \\
-\phi^{+*} & \phi^{0}
\end{array}\right), \quad X=\left(\begin{array}{ccc}
\chi^{0 *} & \xi^{+} & \chi^{++} \\
-\chi^{+*} & \xi^{0} & \chi^{+} \\
\chi^{++*} & -\xi^{+*} & \chi^{0}
\end{array}\right) .
$$

The vevs are defined by $\langle\Phi\rangle=\frac{v_{\phi}}{\sqrt{2}} \mathbb{1}_{2 \times 2}$ and $\langle X\rangle=v_{\chi} \mathbb{1}_{3 \times 3}$, where the $W$ and $Z$ boson masses constrain

$$
v_{\phi}^{2}+8 v_{\chi}^{2} \equiv v^{2}=\frac{4 m_{W}^{2}}{g^{2}} \approx(246 \mathrm{GeV})^{2} .
$$

As discussed in the previous section, the two triplet fields $\chi^{0}$ and $\xi^{0}$ must obtain the same vev $v_{\chi}=v_{\xi}$ in order to preserve custodial $S U(2)_{c}$. Note that after electroweak symmetry breaking (EWSB), the $\mathrm{SU}(2)_{L} \times \mathrm{SU}(2)_{R}$ bidoublet structure of $X$ in Eq. (7.7) is broken down to its diagonal (as required by the $S U(2)_{c}$ custodial symmetry).

The neutral fields may be decomposed into real and imaginary parts,

$$
\phi^{0} \rightarrow \frac{v_{\phi}}{\sqrt{2}}+\frac{\phi^{0, r}+i \phi^{0, i}}{\sqrt{2}}, \quad \chi^{0} \rightarrow v_{\chi}+\frac{\chi^{0, r}+i \chi^{0, i}}{\sqrt{2}}, \quad \xi^{0} \rightarrow v_{\chi}+\xi^{0},
$$

where we note that $\xi^{0}$ is already a real field.

The scalar potential of the GM model has taken different forms in the literature. Several different parametrizations exist, which may be translated from one 
to another using the guide provided in the appendices of Ref. [4]. The most general gauge-invariant scalar potential that still conserves custodial $\mathrm{SU}(2)$ at tree-level is given by

$$
\begin{aligned}
V(\Phi, X)= & \frac{\mu_{2}^{2}}{2} \operatorname{Tr}\left(\Phi^{\dagger} \Phi\right)+\frac{\mu_{3}^{2}}{2} \operatorname{Tr}\left(X^{\dagger} X\right)+\lambda_{1}\left[\operatorname{Tr}\left(\Phi^{\dagger} \Phi\right)\right]^{2}+\lambda_{2} \operatorname{Tr}\left(\Phi^{\dagger} \Phi\right) \operatorname{Tr}\left(X^{\dagger} X\right) \\
& +\lambda_{3} \operatorname{Tr}\left(X^{\dagger} X X^{\dagger} X\right)+\lambda_{4}\left[\operatorname{Tr}\left(X^{\dagger} X\right)\right]^{2}-\lambda_{5} \operatorname{Tr}\left(\Phi^{\dagger} \tau^{a} \Phi \tau^{b}\right) \operatorname{Tr}\left(X^{\dagger} t^{a} X t^{b}\right) \\
& -M_{1} \operatorname{Tr}\left(\Phi^{\dagger} \tau^{a} \Phi \tau^{b}\right)\left(U X U^{\dagger}\right)_{a b}-M_{2} \operatorname{Tr}\left(X^{\dagger} t^{a} X t^{b}\right)\left(U X U^{\dagger}\right)_{a b} .
\end{aligned}
$$

Here the $\mathrm{SU}(2)$ generators for the doublet representation are $\tau^{a}=\sigma^{a} / 2$ (where $\sigma^{a}$ are the Pauli matrices), the generators for the triplet representation are

$$
t^{1}=\frac{1}{\sqrt{2}}\left(\begin{array}{ccc}
0 & 1 & 0 \\
1 & 0 & 1 \\
0 & 1 & 0
\end{array}\right), \quad t^{2}=\frac{1}{\sqrt{2}}\left(\begin{array}{ccc}
0 & -i & 0 \\
i & 0 & -i \\
0 & i & 0
\end{array}\right), \quad t^{3}=\left(\begin{array}{ccc}
1 & 0 & 0 \\
0 & 0 & 0 \\
0 & 0 & -1
\end{array}\right)
$$

and the matrix $U$, which rotates $X$ into the Cartesian basis, is given by [5]

$$
U=\left(\begin{array}{ccc}
-\frac{1}{\sqrt{2}} & 0 & \frac{1}{\sqrt{2}} \\
-\frac{i}{\sqrt{2}} & 0 & -\frac{i}{\sqrt{2}} \\
0 & 1 & 0
\end{array}\right)
$$

As all of the operators in Eq. (7.10) are Hermitian, the parameters in the scalar potential must all be real and explicit CP violation cannot occur.

The potential of Eq. (7.10) was first presented in Ref. [5], and had only been studied in Refs. $[6,144]$ prior to this work. The original presentation of the GM scalar potential imposed an additional $Z_{2}$ symmetry [70], as did much of the subsequent study of the model. This $Z_{2}$ symmetry eliminated the two terms parametrized by dimensionful parameters, such that $M_{1}=M_{2}=0$. This choice was historically made for simplicity; however, it significantly affects the phenomenology of the model, as we will discuss in Sec. 7.3. 
In terms of the vevs, the scalar potential is given by

$$
\begin{aligned}
V\left(v_{\phi}, v_{\chi}\right)= & \frac{\mu_{2}^{2}}{2} v_{\phi}^{2}+3 \frac{\mu_{3}^{2}}{2} v_{\chi}^{2}+\lambda_{1} v_{\phi}^{4}+\frac{3}{2}\left(2 \lambda_{2}-\lambda_{5}\right) v_{\phi}^{2} v_{\chi}^{2} \\
& +3\left(\lambda_{3}+3 \lambda_{4}\right) v_{\chi}^{4}-\frac{3}{4} M_{1} v_{\phi}^{2} v_{\chi}-6 M_{2} v_{\chi}^{3}
\end{aligned}
$$

Minimizing this potential yields the following constraints:

$$
\begin{aligned}
& 0=\frac{\partial V}{\partial v_{\phi}}=v_{\phi}\left[\mu_{2}^{2}+4 \lambda_{1} v_{\phi}^{2}+3\left(2 \lambda_{2}-\lambda_{5}\right) v_{\chi}^{2}-\frac{3}{2} M_{1} v_{\chi}\right] \\
& 0=\frac{\partial V}{\partial v_{\chi}}=3 \mu_{3}^{2} v_{\chi}+3\left(2 \lambda_{2}-\lambda_{5}\right) v_{\phi}^{2} v_{\chi}+12\left(\lambda_{3}+3 \lambda_{4}\right) v_{\chi}^{3}-\frac{3}{4} M_{1} v_{\phi}^{2}-18 M_{2} v_{\chi}^{2}
\end{aligned}
$$

Inserting $v_{\phi}^{2}=v^{2}-8 v_{\chi}^{2}$ (Eq. (7.8)) into Eq. (7.15) yields a cubic equation for $v_{\chi}$ in terms of $v, \mu_{3}^{2}, \lambda_{2}, \lambda_{3}, \lambda_{4}, \lambda_{5}, M_{1}$, and $M_{2}$. With $v_{\chi}$ (and hence $v_{\phi}$ ) in hand, Eq. (7.14) can be used to eliminate $\mu_{2}^{2}$ in terms of these parameters together with $\lambda_{1}$. We illustrate below how $\lambda_{1}$ can also be eliminated in favour of one of the custodial singlet Higgs masses $m_{h}$ or $m_{H}$ (see Eq. (7.36)).

The physical field content is as follows. When expanded around the minimum, the scalar potential gives rise to ten real physical fields together with three Goldstone bosons. After EWSB, the original $S U(2)_{L} \times S U(2)_{R}$ symmetry is broken into the following decomposition: $2 \otimes 2=3 \oplus 1$ and $3 \otimes 3=5 \oplus 3 \oplus 1$. The physical fields can therefore be organized by their transformation properties under the custodial $S U(2)_{c}$ symmetry into a fiveplet, a triplet, and two singlets. The second triplet comprises the Goldstone bosons, which are given by

$$
\begin{aligned}
G^{+} & =c_{H} \phi^{+}+s_{H} \frac{\left(\chi^{+}+\xi^{+}\right)}{\sqrt{2}}, \\
G^{0} & =c_{H} \phi^{0, i}+s_{H} \chi^{0, i},
\end{aligned}
$$

where

$$
c_{H} \equiv \cos \theta_{H}=\frac{v_{\phi}}{v}, \quad s_{H} \equiv \sin \theta_{H}=\frac{2 \sqrt{2} v_{\chi}}{v}
$$


The physical fiveplet and triplet states are given by

$$
\begin{aligned}
H_{5}^{++} & =\chi^{++} \\
H_{5}^{+} & =\frac{\left(\chi^{+}-\xi^{+}\right)}{\sqrt{2}}, \\
H_{5}^{0} & =\sqrt{\frac{2}{3}} \xi^{0}-\sqrt{\frac{1}{3}} \chi^{0, r}, \\
H_{3}^{+} & =-s_{H} \phi^{+}+c_{H} \frac{\left(\chi^{+}+\xi^{+}\right)}{\sqrt{2}}, \\
H_{3}^{0} & =-s_{H} \phi^{0, i}+c_{H} \chi^{0, i} .
\end{aligned}
$$

Note that only the $H_{3}$ triplet states include fields from the doublet $\Phi$, while the $H_{5}$ states are entirely composed of triplet fields from $\chi$ and $\xi$. As fermions couple only to $\Phi$ (due to dimension), the $H_{3}$ states may couple to fermions, but the $H_{5}$ states will not (i.e. they are fermiophobic, $C_{H_{5} \bar{f} f}=0$ ). Furthermore, the $H_{3}$ states will not be able to couple to two vector bosons (i.e. they are fermiophilic, $C_{H_{3} V V}=0$ ).

Within each custodial multiplet, the masses are degenerate at tree level. Using Eqs. (7.14-7.15) to eliminate $\mu_{2}^{2}$ and $\mu_{3}^{2}$, the fiveplet and triplet masses can be written as

$$
\begin{aligned}
& m_{5}^{2}=\frac{M_{1}}{4 v_{\chi}} v_{\phi}^{2}+12 M_{2} v_{\chi}+\frac{3}{2} \lambda_{5} v_{\phi}^{2}+8 \lambda_{3} v_{\chi}^{2} \\
& m_{3}^{2}=\frac{M_{1}}{4 v_{\chi}}\left(v_{\phi}^{2}+8 v_{\chi}^{2}\right)+\frac{\lambda_{5}}{2}\left(v_{\phi}^{2}+8 v_{\chi}^{2}\right)=\left(\frac{M_{1}}{4 v_{\chi}}+\frac{\lambda_{5}}{2}\right) v^{2} .
\end{aligned}
$$

Note that the ratio $M_{1} / v_{\chi}$ is finite in the limit $v_{\chi} \rightarrow 0$, as can be seen from Eq. (7.15) which yields

$$
\frac{M_{1}}{v_{\chi}}=\frac{4}{v_{\phi}^{2}}\left[\mu_{3}^{2}+\left(2 \lambda_{2}-\lambda_{5}\right) v_{\phi}^{2}+4\left(\lambda_{3}+3 \lambda_{4}\right) v_{\chi}^{2}-6 M_{2} v_{\chi}\right] .
$$

The two custodial SU(2) singlets are given in the gauge basis by $\phi^{0, r}$ and

$$
H_{1}^{0 \prime}=\sqrt{\frac{1}{3}} \xi^{0}+\sqrt{\frac{2}{3}} \chi^{0, r} .
$$


These states mix by an angle $\alpha$ to form the two custodial-singlet mass eigenstates $h$ and $H$, defined such that $m_{h}<m_{H}$ :

$$
\begin{gathered}
h=\cos \alpha \phi^{0, r}-\sin \alpha H_{1}^{0 \prime}, \\
H=\sin \alpha \phi^{0, r}+\cos \alpha H_{1}^{0 \prime} .
\end{gathered}
$$

The mixing is controlled by the $2 \times 2$ mass-squared matrix

$$
\mathcal{M}^{2}=\left(\begin{array}{ll}
\mathcal{M}_{11}^{2} & \mathcal{M}_{12}^{2} \\
\mathcal{M}_{12}^{2} & \mathcal{M}_{22}^{2}
\end{array}\right),
$$

where

$$
\begin{aligned}
& \mathcal{M}_{11}^{2}=8 \lambda_{1} v_{\phi}^{2}, \\
& \mathcal{M}_{12}^{2}=\frac{\sqrt{3}}{2} v_{\phi}\left[-M_{1}+4\left(2 \lambda_{2}-\lambda_{5}\right) v_{\chi}\right], \\
& \mathcal{M}_{22}^{2}=\frac{M_{1} v_{\phi}^{2}}{4 v_{\chi}}-6 M_{2} v_{\chi}+8\left(\lambda_{3}+3 \lambda_{4}\right) v_{\chi}^{2} .
\end{aligned}
$$

The mixing angle is fixed by

$$
\begin{aligned}
\sin 2 \alpha & =\frac{2 \mathcal{M}_{12}^{2}}{m_{H}^{2}-m_{h}^{2}}, \\
\cos 2 \alpha & =\frac{\mathcal{M}_{22}^{2}-\mathcal{M}_{11}^{2}}{m_{H}^{2}-m_{h}^{2}},
\end{aligned}
$$

with the masses given by

$$
m_{h, H}^{2}=\frac{1}{2}\left[\mathcal{M}_{11}^{2}+\mathcal{M}_{22}^{2} \mp \sqrt{\left(\mathcal{M}_{11}^{2}-\mathcal{M}_{22}^{2}\right)^{2}+4\left(\mathcal{M}_{12}^{2}\right)^{2}}\right] .
$$

It is convenient to use the measured $125 \mathrm{GeV}$ mass of the observed SM-like Higgs boson as an input parameter. The coupling $\lambda_{1}$ can be eliminated in favour of this mass by inverting Eq. (7.35):

$$
\lambda_{1}=\frac{1}{8 v_{\phi}^{2}}\left[m_{h}^{2}+\frac{\left(\mathcal{M}_{12}^{2}\right)^{2}}{\mathcal{M}_{22}^{2}-m_{h}^{2}}\right] .
$$


Note that in deriving this expression for $\lambda_{1}$, the distinction between $m_{h}$ and $m_{H}$ is lost. This means that, depending on the values of $\mu_{3}^{2}$ and the other parameters, this (unique) solution for $\lambda_{1}$ will correspond to either the lighter or the heavier custodial singlet having a mass equal to the observed SM-like Higgs mass.

\subsection{Theoretical constraints}

\subsubsection{Constraints from tree-level perturbative unitarity}

The scalar quartic couplings $\lambda_{i}(1 \leq i \leq 5)$ given in Eq. (7.10) can be bounded by requiring tree-level perturbative unitarity of the zeroth partial-wave scattering amplitudes according to Eq. (3.2). The dimensionful couplings $M_{1}, M_{2}, \mu_{2}^{2}$, and $\mu_{3}^{2}$ are not constrained directly by perturbative unitarity, as diagrams involving scalar propagators are suppressed by the square of the collision energy and neglected in the high-energy limit. In this limit we calculate the $S S \rightarrow S S$ amplitudes from quartic interactions (given by Eq. (3.4)), including Goldstone bosons in the place of longitudinal polarizations of the gauge bosons. However, we neglect scattering processes involving transversely polarized gauge bosons; this is equivalent to our approximation in previous chapters of neglecting amplitudes proportional to the weak coupling which were not $n$-enhanced. Our independent calculation of these unitarity bounds agrees with the results of Ref. [5], where they were originally presented.

As before, the $\mathrm{SU}(2)_{L} \times \mathrm{U}(1)_{Y}$-symmetric potential allows us to group the amplitudes into matrices by the total isospin and hypercharge of the initial and final states. These amplitude matrices provide nine distinct eigenvalues, all of which may be determined by considering the matrix of electrically neutral amplitudes with 
$Y=0,1$. Additional interactions with higher $Y$ or $Q \neq 0$ simply reproduce various subsets of the same nine eigenvalues, as seen in Table 7.1.

Neutral amplitudes with $Y=0$

The neutral amplitudes with total hypercharge of zero form a $7 \times 7$ matrix in the basis

$$
\left[\chi^{--} \chi^{++}, \chi^{-} \chi^{+}, \xi^{-} \xi^{+}, \phi^{-} \phi^{+}, \chi^{0 *} \chi^{0}, \xi^{0} \xi^{0} / \sqrt{2}, \phi^{0 *} \phi^{0}\right]
$$

As indicated by by Eq. (3.4), the elements of this matrix will be the quartic scalar couplings $C_{s_{1} s_{2} s_{3} s_{4}}$, which may be read directly from Eq. (7.10) when expanded in terms of the individual fields. Using Eqs. (3.15-3.17), this $7 \times 7$ matrix may be simplified into three smaller matrices by grouping the initial and final states by total isospin, resulting in a $3 \times 3$ isospin- 0 matrix, a $2 \times 2$ isospin- 1 matrix, and a $2 \times 2$ isospin-2 matrix.

The linear combinations of scalar states with total isospin zero are given by

$$
\begin{aligned}
\left(\phi^{*} \phi\right)_{0} & =\frac{1}{\sqrt{2}}\left(\phi^{0 *} \phi^{0}+\phi^{+*} \phi^{+}\right) \\
\left(\chi^{*} \chi\right)_{0} & =\frac{1}{\sqrt{3}}\left(\chi^{0 *} \chi^{0}+\chi^{+*} \chi^{+}+\chi^{++*} \chi^{++}\right) \\
\left(\xi^{*} \xi\right)_{0} & =\frac{1}{\sqrt{3}}\left(\sqrt{2} \xi^{+*} \xi^{+}+\frac{\xi^{0} \xi^{0}}{\sqrt{2}}\right)
\end{aligned}
$$

where $\left(\xi^{*} \xi\right)_{0}$ includes the symmetry factor of $1 / \sqrt{2}$ for identical particles. Then the total-isospin-0, hypercharge-0 coupled-channel matrix $\mathcal{M}$ for neutral amplitudes in the basis $\left[\left(\phi^{*} \phi\right)_{0},\left(\xi^{*} \xi\right)_{0},\left(\chi^{*} \chi\right)_{0}\right]$ is

$$
\mathcal{M}_{0}^{(0,0)}=\left(\begin{array}{ccc}
24 \lambda_{1} & 4 \sqrt{3} \lambda_{2} & 4 \sqrt{6} \lambda_{2} \\
4 \sqrt{3} \lambda_{2} & 20\left(\lambda_{3}+\lambda_{4}\right) & 4 \sqrt{2}\left(\lambda_{3}+3 \lambda_{4}\right) \\
4 \sqrt{6} \lambda_{2} & 4 \sqrt{2}\left(\lambda_{3}+3 \lambda_{4}\right) & 8\left(3 \lambda_{3}+4 \lambda_{4}\right)
\end{array}\right)
$$


where the subscript in $\mathcal{M}_{0}^{(Y, T)}$ indicates that the matrix contains the amplitudes contributing to $a_{0}$ (see Eqs. (3.1) and (3.3)), and the superscript indicates the total hypercharge $Y$ and isospin $T$. This matrix has three eigenvalues given by

$$
\begin{aligned}
& x_{1}^{ \pm}=12 \lambda_{1}+14 \lambda_{3}+22 \lambda_{4} \pm \sqrt{\left(12 \lambda_{1}-14 \lambda_{3}-22 \lambda_{4}\right)^{2}+144 \lambda_{2}^{2}}, \\
& y_{1}=16 \lambda_{3}+8 \lambda_{4}
\end{aligned}
$$

which have been defined to be consistent with Ref. [5]. ${ }^{2}$ The unitarity bound on the zeroth partial wave $a_{0}$ therefore requires that

$$
\left|a_{0}^{(0,0), \max }\right|=\frac{1}{16 \pi} \max \left\{\left|x_{1}^{+}\right|,\left|x_{1}^{-}\right|,\left|y_{1}\right|\right\} \leq \frac{1}{2}
$$

As the maximum of these eigenvalues will vary with the values of $\lambda_{i}$, in general the preservation of the perturbative unitarity bound requires that the parameters $\lambda_{i}$ satisfy $\left|x_{1}^{ \pm}\right| \leq 8 \pi$ and $\left|y_{1}\right| \leq 8 \pi$.

The linear combinations of scalar states with total isospin 1 are given by

$$
\begin{aligned}
\left(\phi^{*} \phi\right)_{1} & =\frac{1}{\sqrt{2}}\left(\phi^{0 *} \phi^{0}-\phi^{+*} \phi^{+}\right), \\
\left(\chi^{*} \chi\right)_{1} & =\frac{1}{\sqrt{2}}\left(\chi^{0 *} \chi^{0}-\chi^{++*} \chi^{++}\right),
\end{aligned}
$$

resulting in the following coupled-channel matrix in the basis $\left[\left(\phi^{*} \phi\right)_{1},\left(\xi^{*} \xi\right)_{1}\right]$,

$$
\mathcal{M}_{0}^{(0,1)}=\left(\begin{array}{cc}
8 \lambda_{1} & -2 \lambda_{5} \\
-2 \lambda_{5} & 4\left(-\lambda_{3}+2 \lambda_{4}\right)
\end{array}\right),
$$

which has two eigenvalues,

$$
x_{2}^{ \pm}=4 \lambda_{1}-2 \lambda_{3}+4 \lambda_{4} \pm \sqrt{\left(4 \lambda_{1}+2 \lambda_{3}-4 \lambda_{4}\right)^{2}+4 \lambda_{5}^{2}}
$$

\footnotetext{
${ }^{2}$ Our notation for the $\lambda_{i}$ is different from that of Ref. [5]. This has been taken into account in the definitions of $x_{i}^{ \pm}$and $y_{i}$ in Eq. (7.56). A translation between our notation and that of Ref. [5] is given in the appendix of Ref. [4].
} 
resulting in constraints on $\lambda_{i}$ defined by $\left|x_{2}^{ \pm}\right| \leq 8 \pi$.

The linear combinations of scalar states with total isospin 2 are given by

$$
\begin{aligned}
\left(\chi^{*} \chi\right)_{2} & =\frac{1}{\sqrt{6}}\left(-\chi^{0 *} \chi^{0}+2 \chi^{+*} \chi^{+}-\chi^{++*} \chi^{++}\right) \\
\left(\xi^{*} \xi\right)_{2} & =\frac{1}{\sqrt{3}}\left(-\xi^{+*} \xi^{+}+\sqrt{2} \frac{\xi^{0} \xi^{0}}{\sqrt{2}}\right)
\end{aligned}
$$

where $\left(\xi^{*} \xi\right)_{2}$ already includes the appropriate symmetry factor of $1 / \sqrt{2}$. This leads to the following matrix in the basis $\left[\left(\xi^{*} \xi\right)_{2},\left(\chi^{*} \chi\right)_{2}\right]$,

$$
\mathcal{M}_{0}^{(0,2)}=\left(\begin{array}{cc}
8\left(\lambda_{3}+\lambda_{4}\right) & 4 \sqrt{2} \lambda_{3} \\
4 \sqrt{2} \lambda_{3} & 4\left(3 \lambda_{3}+2 \lambda_{4}\right)
\end{array}\right)
$$

which has two eigenvalues, the $y_{1}$ defined by Eq. (7.43) and

$$
y_{2}=4 \lambda_{3}+8 \lambda_{4}
$$

Due to the duplicate eigenvalue, the isospin-2 matrix therefore produces a single additional bound of $\left|y_{2}\right| \leq 8 \pi$.

\section{Neutral amplitudes with $Y=1$}

The matrix of neutral coupled channel amplitudes with total hypercharge of 1 is given in the basis of $\left[\phi^{+} \xi^{+*}, \phi^{0} \xi^{0}, \chi^{+} \phi^{+*}, \chi^{0} \phi^{0 *}\right]$ by

$$
\mathcal{M}_{0}^{(1)}=\left(\begin{array}{cccc}
4 \lambda_{2} & -2 \lambda_{5} & -\sqrt{2} \lambda_{5} & 0 \\
-2 \lambda_{5} & 4 \lambda_{2} & -\sqrt{2} \lambda_{5} & 0 \\
-\sqrt{2} \lambda_{5} & -\sqrt{2} \lambda_{5} & 4 \lambda_{2}-\lambda_{5} & -2 \lambda_{5} \\
0 & 0 & -2 \lambda_{5} & 4 \lambda_{2}
\end{array}\right)
$$


where here the superscript on $\mathcal{M}$ indicates the total hypercharge $Y=1$. This matrix has the following eigenvalues:

$$
\begin{aligned}
& y_{3}=4 \lambda_{2}-\lambda_{5}, \\
& y_{4}=4 \lambda_{2}+2 \lambda_{5}, \\
& y_{5}=4 \lambda_{2}-4 \lambda_{5},
\end{aligned}
$$

where $y_{4}$ occurs twice, resulting in three new bounds on the coupling parameters defined by $\left|y_{i}\right| \leq 8 \pi$ for $i=3,4,5$.

\section{Maximum parameter ranges allowed by perturbative unitarity}

The bounds from the remaining amplitudes of other total $Q$ and $Y$ were calculated by a similar procedure, and the results for all interactions are summarized in Table 7.1. A symmetry factor of $1 / \sqrt{2}$ is included for each pair of identical particles in the initial and final states. We have not listed the charge-conjugates of the states as they yield the same sets of eigenvalues.

In general the preservation of perturbative unitarity requires that $\lambda_{i}$ simultaneously satisfy the conditions

$$
\left|x_{i}^{ \pm}\right| \leq 8 \pi \quad \text { and } \quad\left|y_{i}\right| \leq 8 \pi .
$$

Note that the bounds on $y_{1,2}$ constrain $\lambda_{3,4}$, the bounds on $y_{3,4}$ constrain $\lambda_{2,5}$, the

bounds on $x_{1}^{ \pm}$constrain $\lambda_{1,2,3,4}$, and the bounds on $x_{2}^{ \pm}$constrain $\lambda_{1,3,4,5}$. Discarding expressions that provide no additional information, we find that the minimal set of 


\begin{tabular}{|c|c|c|c|}
\hline$Q$ & $Y$ & Basis states & Eigenvalues \\
\hline 0 & 0 & {$\left[\chi^{++*} \chi^{++}, \chi^{+*} \chi^{+}, \xi^{+*} \xi^{+}, \phi^{+*} \phi^{+}, \chi^{0 *} \chi^{0}, \frac{\xi^{0} \xi^{0}}{\sqrt{2}}, \phi^{0 *} \phi^{0}\right]$} & $x_{1}^{+}, x_{1}^{-}, x_{2}^{+}, x_{2}^{-}, y_{1}, y_{1}, y_{2}$ \\
\hline 0 & 1 & {$\left[\phi^{+} \xi^{+*}, \phi^{0} \xi^{0}, \chi^{+} \phi^{+*}, \chi^{0} \phi^{0 *}\right]$} & $y_{3}, y_{4}, y_{4}, y_{5}$ \\
\hline 0 & 2 & {$\left[\frac{\phi^{0} \phi^{0}}{\sqrt{2}}, \chi^{0} \xi^{0}, \chi^{+} \xi^{+*}\right]$} & $x_{2}^{+}, x_{2}^{-}, y_{2}$ \\
\hline 0 & 3 & {$\left[\phi^{0} \chi^{0}\right]$} & $y_{3}$ \\
\hline 0 & 4 & {$\left[\frac{\chi^{0} \chi^{0}}{\sqrt{2}}\right]$} & $y_{2}$ \\
\hline 1 & -2 & {$\left[\xi^{+} \chi^{0 *}\right]$} & $y_{2}$ \\
\hline 1 & -1 & {$\left[\phi^{+} \chi^{0 *}, \xi^{+} \phi^{0 *}\right]$} & $y_{3}, y_{4}$ \\
\hline 1 & 0 & {$\left[\xi^{+} \xi^{0}, \chi^{+*} \chi^{++}, \phi^{+} \phi^{0 *}, \chi^{0 *} \chi^{+}\right]$} & $x_{2}^{+}, x_{2}^{-}, y_{1}, y_{2}$ \\
\hline 1 & 1 & {$\left[\phi^{0} \xi^{+}, \phi^{+} \xi^{0}, \phi^{+*} \chi^{++}, \phi^{0 *} \chi^{+}\right]$} & $y_{3}, y_{4}, y_{4}, y_{5}$ \\
\hline 1 & 2 & {$\left[\phi^{+} \phi^{0}, \chi^{+} \xi^{0}, \chi^{++} \xi^{+*}, \chi^{0} \xi^{+}\right]$} & $x_{2}^{+}, x_{2}^{-}, y_{1}, y_{2}$ \\
\hline 1 & 3 & {$\left[\phi^{+} \chi^{0}, \phi^{0} \chi^{+}\right]$} & $y_{3}, y_{4}$ \\
\hline 1 & 4 & {$\left[\chi^{+} \chi^{0}\right]$} & $y_{2}$ \\
\hline 2 & 0 & {$\left[\chi^{++} \chi^{0 *}, \frac{\xi^{+} \xi^{+}}{\sqrt{2}}\right]$} & $y_{1}, y_{2}$ \\
\hline 2 & 1 & {$\left[\phi^{+} \xi^{+}, \chi^{++} \phi^{0 *}\right]$} & $y_{3}, y_{4}$ \\
\hline 2 & 2 & {$\left[\frac{\phi^{+} \phi^{+}}{\sqrt{2}}, \chi^{++} \xi^{0}, \chi^{+} \xi^{+}\right]$} & $x_{2}^{+}, x_{2}^{-}, y_{2}$ \\
\hline 2 & 3 & {$\left[\phi^{+} \chi^{+}, \phi^{0} \chi^{++}\right]$} & $y_{3}, y_{4}$ \\
\hline 2 & 4 & {$\left[\chi^{++} \chi^{0}, \frac{\chi^{+} \chi^{+}}{\sqrt{2}}\right]$} & $y_{1}, y_{2}$ \\
\hline 3 & 2 & {$\left[\chi^{++} \xi^{+}\right]$} & $y_{2}$ \\
\hline 3 & 3 & {$\left[\chi^{++} \phi^{+}\right]$} & $y_{3}$ \\
\hline 3 & 4 & {$\left[\chi^{++} \chi^{+}\right]$} & $y_{2}$ \\
\hline 4 & 4 & {$\left[\frac{\chi^{++} \chi^{++}}{\sqrt{2}}\right]$} & $y_{2}$ \\
\hline
\end{tabular}

Table 7.1: Basis states and eigenvalues of the scattering matrix $\mathcal{M}$ for $2 \rightarrow 2$ scalar scattering in the high-energy limit, classified according to the total charge $Q$ and total hypercharge $Y$ of the initial and final states. We have included a symmetry factor of $1 / \sqrt{2}$ in the matrix element for each pair of identical particles in the initial or final state. The eigenvalues are defined in Eq. (7.56). The charge-conjugates of the listed states yield the same sets of eigenvalues. 
unitarity conditions are those given by $x_{1,2}^{ \pm}, y_{4}$, and $y_{5}$,

$$
\begin{aligned}
\sqrt{\left(6 \lambda_{1}-7 \lambda_{3}-11 \lambda_{4}\right)^{2}+36 \lambda_{2}^{2}}+\left|6 \lambda_{1}+7 \lambda_{3}+11 \lambda_{4}\right| & <4 \pi \\
\sqrt{\left(2 \lambda_{1}+\lambda_{3}-2 \lambda_{4}\right)^{2}+\lambda_{5}^{2}}+\left|2 \lambda_{1}-\lambda_{3}+2 \lambda_{4}\right| & <4 \pi \\
\left|2 \lambda_{3}+\lambda_{4}\right| & <\pi \\
\left|\lambda_{2}-\lambda_{5}\right| & <2 \pi
\end{aligned}
$$

The constraints from $y_{2,3,4}$ do not provide any additional information.

The conditions of Eq. (7.57) allow us to determine the maximum range allowed by unitarity for each of the parameters $\lambda_{i}$, which facilitates efficient numerical scanning of the parameter space. We first note that the conditions $\left|x_{i}^{ \pm}\right|<8 \pi$ take the general form $\left|x+z \pm \sqrt{(x-z)^{2}+y^{2}}\right|<1$, where $x$ is proportional to $\lambda_{1}, z$ is made up of $\lambda_{3,4}$, and $y$ is made of $\lambda_{2,5}$. As the contributions $(x-z)$ and $y$ are added in quadrature, the maximum allowed value of one will occur when the other is set to zero, i.e. $\lambda_{1,3,4}$ will be maximized when $\lambda_{2,5}=0$ and vice versa. ${ }^{3}$

The coupling $\lambda_{1}$ is constrained by the unitarity conditions on $x_{1}^{ \pm}$and $x_{2}^{ \pm}$. The least stringent constraints come from setting $\lambda_{2}=\lambda_{5}=0$; in this case the form $\left|x+z \pm \sqrt{(x-z)^{2}+y^{2}}\right|<1$ reduces to $|x+z \pm(x-z)|<1$ and the $\lambda_{1}$ component $(x)$ is isolated by $x_{1,2}^{+}$. The bounds $\left|\lambda_{1}\right|<\frac{1}{3} \pi$ and $\left|\lambda_{1}\right|<\pi$ can respectively be read from $x_{1}^{+}$and $x_{2}^{+}$. We thus obtain the maximum range from unitarity,

$$
\lambda_{1} \in\left(-\frac{1}{3} \pi, \frac{1}{3} \pi\right) \simeq(-1.05,1.05)
$$

Note that $\lambda_{1}$ is not a free parameter, but is fixed by the known value of the Higgs mass $m_{h}=125 \mathrm{GeV}$ in terms of the parameters $\lambda_{2,3,4,5}, M_{1,2}$ (see Eq. (7.36)). As a

\footnotetext{
${ }^{3}$ Alternatively, $\left|a \pm \sqrt{b^{2}+c^{2}}\right|<1$ can be equivalently written as $\sqrt{b^{2}+c^{2}}+|a|<1$, which describes a pair of cones with apices at $a= \pm 1$ that meet at a unit circle in the $(b, c)$ plane. Therefore the maximum values of $b$ and $c$ will occur when $a=0$, and vice versa.
} 
result, the unitarity bound on $\lambda_{1}$ actually constrains the dimensionful parameter $M_{1}$. By Taylor expanding Eq. (7.36) in the limit that $\mu_{3}$ becomes very heavy $\left(\mu_{3}^{2} \gg \lambda_{i} v^{2}\right)$ and $v_{\chi}$ approaches zero, one can show ${ }^{4}$ that

$$
\lambda_{1}=\frac{1}{8}\left[\frac{m_{h}^{2}}{v^{2}}+\frac{3}{4} \frac{M_{1}^{2}}{\mu_{3}^{2}}+\ldots\right]
$$

Applying the unitarity bound from Eq. (7.59) and evaluating at $m_{h}=125 \mathrm{GeV}$, we find that $M_{1}$ can scale at most linearly with $\sqrt{\mu_{3}^{2}}$ and that its value is constrained by $\left|M_{1}\right| / \sqrt{\mu_{3}^{2}} \lesssim 3.3$

Constraints on the couplings $\lambda_{3}$ and $\lambda_{4}$ come from the unitarity conditions on $x_{1}^{ \pm}, x_{2}^{ \pm}, y_{1}$. Similarly to the $\lambda_{1}$ case, the $x_{1,2}^{-}$bounds reduce to linear combinations of $\lambda_{3}$ and $\lambda_{4}$ when $\lambda_{2}=\lambda_{5}=0$. As the bounds from $y_{1,2}$ are similarly linear in $\lambda_{3}$ and $\lambda_{4}$, the least stringent bounds may be represented by straight lines in the $\left(\lambda_{3}, \lambda_{4}\right)$ plane, as shown in the left panel of Fig. 7.1. The resulting allowed parameter space in the $\left(\lambda_{3}, \lambda_{4}\right)$ plane is a six-sided region bounded by the constraints on $x_{1}^{-}, x_{2}^{-}$, and $y_{1}$. Simultaneously satisfying all constraints, we obtain the maximum ranges from unitarity,

$$
\begin{aligned}
& \lambda_{3} \in\left(-\frac{4}{5} \pi, \frac{4}{5} \pi\right) \simeq(-2.51,2.51) \\
& \lambda_{4} \in\left(-\frac{16}{25} \pi, \frac{16}{25} \pi\right) \simeq(-2.01,2.01)
\end{aligned}
$$

Constraints on the couplings $\lambda_{2}$ and $\lambda_{5}$ come from the unitarity constraints on $x_{1}^{ \pm}, x_{2}^{ \pm}$, and $y_{5}$. These are shown in the right panel of Fig. 7.1, where we take $\lambda_{1}=\lambda_{3}=\lambda_{4}=0$ in $x_{1}^{ \pm}$and $x_{2}^{ \pm}$for the least stringent constraints. (The constraint from $x_{2}^{ \pm}$yields $\left|\lambda_{5}\right|<4 \pi$, which corresponds to the left and right edges of the plot.) The allowed region in the $\left(\lambda_{2}, \lambda_{5}\right)$ plane is a parallelogram bounded by the constraints

\footnotetext{
${ }^{4}$ The details of this expansion may be found in Sec. 7.3.
} 

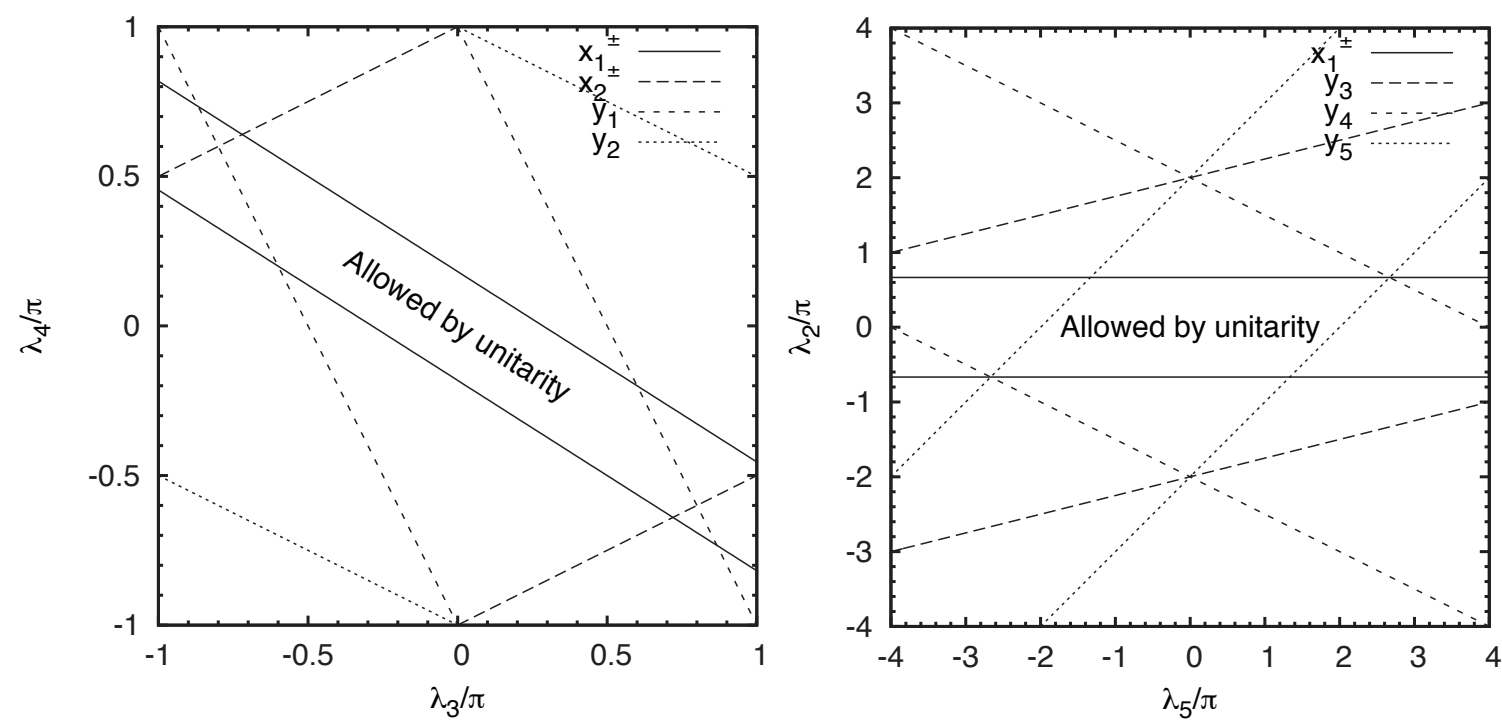

Figure 7.1: Constraints on the $\left(\lambda_{3}, \lambda_{4}\right)$ and $\left(\lambda_{5}, \lambda_{2}\right)$ planes from perturbative unitarity. The constraints from $x_{1}^{ \pm}$and $x_{2}^{ \pm}$are the maximum allowed ranges obtained by setting the couplings not shown on the figure axes to zero.

on $x_{1}^{ \pm}$and $y_{5}$. Simultaneously satisfying all constraints, we obtain the maximum ranges from unitarity,

$$
\begin{aligned}
& \lambda_{2} \in\left(-\frac{2}{3} \pi, \frac{2}{3} \pi\right) \simeq(-2.09,2.09) \\
& \lambda_{5} \in\left(-\frac{8}{3} \pi, \frac{8}{3} \pi\right) \simeq(-8.38,8.38)
\end{aligned}
$$

Within these maximum ranges the unitarity constraints of Eq. (7.58) must still be imposed.

\subsubsection{Bounded-from-below constraints}

As discussed in Sec. 3.1.2, the constraints that must be satisfied at tree level for the scalar potential to be bounded from below can most easily be determined by reparametrizing the terms in the scalar potential that are quartic in the scalar fields, as these terms dominate at large field values. As the quartic terms in Eq. (7.10) 
are parametrized by $\lambda_{i}(1 \leq i \leq 5)$, these are the parameters that will be limited by bounded-from-below (BFB) constraints. Following the approach of Ref. [69] as outlined in Sec. 3.1.2, we reparametrize the potential using the following definitions:

$$
\begin{aligned}
r & \equiv \sqrt{\operatorname{Tr}\left(\Phi^{\dagger} \Phi\right)+\operatorname{Tr}\left(X^{\dagger} X\right)}, \\
r^{2} \cos ^{2} \gamma & \equiv \operatorname{Tr}\left(\Phi^{\dagger} \Phi\right), \\
r^{2} \sin ^{2} \gamma & \equiv \operatorname{Tr}\left(X^{\dagger} X\right), \\
\zeta & \equiv \frac{\operatorname{Tr}\left(X^{\dagger} X X^{\dagger} X\right)}{\left[\operatorname{Tr}\left(X^{\dagger} X\right)\right]^{2}}, \\
\omega & \equiv \frac{\operatorname{Tr}\left(\Phi^{\dagger} \tau^{a} \Phi \tau^{b}\right) \operatorname{Tr}\left(X^{\dagger} t^{a} X t^{b}\right)}{\operatorname{Tr}\left(\Phi^{\dagger} \Phi\right) \operatorname{Tr}\left(X^{\dagger} X\right)} .
\end{aligned}
$$

The quartic terms in the potential are given in this parametrization by,

$$
V^{(4)}(r, \gamma, \zeta, \omega)=\frac{r^{4}}{\left(1+\tan ^{2} \gamma\right)^{2}}\left[\lambda_{1}+\left(\lambda_{2}-\omega \lambda_{5}\right) \tan ^{2} \gamma+\left(\zeta \lambda_{3}+\lambda_{4}\right) \tan ^{4} \gamma\right]
$$

The potential will be bounded from below if the expression in the square brackets in Eq. (7.69) is always positive. This expression is a bi-quadratic in $\tan \gamma \equiv y$ of the form $a+b y^{2}+c y^{4}$, which is positive for all values of $y \in[0, \infty)^{5}$ when $a>0, c>0$, and $b+2 \sqrt{a c}>0$. We thus obtain the following BFB conditions,

$$
\lambda_{1}>0, \quad \zeta \lambda_{3}+\lambda_{4}>0, \quad \text { and } \quad \lambda_{2}-\omega \lambda_{5}+2 \sqrt{\lambda_{1}\left(\zeta \lambda_{3}+\lambda_{4}\right)}>0 .
$$

These conditions must be satisfied for all allowed values of $\zeta$ and $\omega$.

\section{Maximal ranges of the parametrization variables}

As the scalar fields may have any magnitude from zero to infinity, it is evident from Eq. (7.64) that the dimensionful variable $r$ must be positive and may have a similar

\footnotetext{
${ }^{5}$ The parameter range of $\gamma \in[0, \pi / 2]$ can be determined by considering Eqs. (7.65) and (7.66) with all possible field values, either analytically or with a parameter scan.
} 
magnitude, $r \in[0, \infty)$. Similarly, as the traces $\operatorname{Tr}\left(\Phi^{\dagger} \Phi\right)$ and $\operatorname{Tr}\left(X^{\dagger} X\right)$ must also be positive, $\gamma$ may be defined to have range $\gamma \in\left[0, \frac{\pi}{2}\right]$, so that $y \equiv \tan \gamma \in[0, \infty)$. However, determining the possible ranges of $\zeta$ and $\omega$ is more complicated.

The field combination $\zeta$ defined in Eq. (7.67) is given explicitly by

$$
\begin{aligned}
\zeta= & \frac{1}{\left[\operatorname{Tr}\left(X^{\dagger} X\right)\right]^{2}}\left[2\left(\left|\chi^{0}\right|^{2}+\left|\chi^{+}\right|^{2}+\left|\chi^{++}\right|^{2}\right)^{2}+\left(2\left|\xi^{+}\right|^{2}+\left(\xi^{0}\right)^{2}\right)^{2}\right. \\
& \left.+2\left|\chi^{+} \chi^{+}-2 \chi^{0} \chi^{++}\right|^{2}+4\left|\xi^{+} \chi^{0}-\xi^{0} \chi^{+}-\xi^{+*} \chi^{++}\right|^{2}\right]
\end{aligned}
$$

where

$$
\operatorname{Tr}\left(X^{\dagger} X\right)=2\left|\chi^{0}\right|^{2}+2\left|\chi^{+}\right|^{2}+2\left|\chi^{++}\right|^{2}+2\left|\xi^{+}\right|^{2}+\left(\xi^{0}\right)^{2}
$$

To derive the allowed range of $\zeta$, we can work in a basis where the Hermitian matrix $X^{\dagger} X$ is diagonalized with positive real eigenvalues $x_{1}, x_{2}$ and $x_{3}$. In this basis,

$$
\zeta=\frac{x_{1}^{2}+x_{2}^{2}+x_{3}^{2}}{x_{1}^{2}+x_{2}^{2}+x_{3}^{2}+2\left(x_{1} x_{2}+x_{2} x_{3}+x_{3} x_{1}\right)}=\left[1+\frac{2\left(x_{1} x_{2}+x_{2} x_{3}+x_{3} x_{1}\right)}{x_{1}^{2}+x_{2}^{2}+x_{3}^{2}}\right]^{-1} .
$$

We can see that $\zeta$ will have a maximum value of 1 when the second term on the right of Eq. (7.71) goes to zero (e.g. if any two of the $x_{i}$ are set to zero). Similarly, using $a^{2}+b^{2} \geq 2 a b$, we may also see that $\zeta \geq 1 / 3$. We therefore find that $\zeta$ must fall in the range $\zeta \in\left[\frac{1}{3}, 1\right]$. By substituting $\left\langle\chi^{0}\right\rangle=\left\langle\xi^{0}\right\rangle=v_{\chi}$ into Eq. (7.71) and setting all other fields to zero, one will note that the desired electroweak symmetry breaking vacuum corresponds to $\zeta=1 / 3$.

To derive the allowed range of $\omega$, we can start by choosing the $\mathrm{SU}(2)_{L}$ basis so that the field value of $\Phi$ lies entirely in the real neutral component, $\Phi=\frac{v_{\phi}}{\sqrt{2}} \mathbb{1}_{2 \times 2}$. Then,

$$
\frac{\operatorname{Tr}\left(\Phi^{\dagger} \tau^{a} \Phi \tau^{b}\right)}{\operatorname{Tr}\left(\Phi^{\dagger} \Phi\right)}=\frac{1}{4} \delta^{a b}
$$

Inserting this into the expression for $\omega$ in Eq. (7.68) yields

$$
\omega=\frac{1}{2 \operatorname{Tr}\left(X^{\dagger} X\right)}\left[\left|\chi^{0}\right|^{2}-\left|\chi^{++}\right|^{2}+2 \xi^{0} \operatorname{Re} \chi^{0}+2 \operatorname{Re}\left(\xi^{+} \chi^{+*}\right)\right] .
$$


Because $\omega$ is invariant under custodial $\mathrm{SU}(2)$, this expression can be rewritten in terms of custodial $\mathrm{SU}(2)$ eigenstates as follows. We first define the custodial singlet, triplet, and fiveplet contained in $X$ according to

$$
X_{1}=\frac{\xi^{0}+2 \operatorname{Re} \chi^{0}}{\sqrt{3}}, \quad X_{3}=\left(\begin{array}{c}
\frac{1}{\sqrt{2}}\left(\chi^{+}+\xi^{+}\right) \\
\sqrt{2} \operatorname{Im} \chi^{0} \\
-\frac{1}{\sqrt{2}}\left(\chi^{+*}+\xi^{+*}\right)
\end{array}\right), \quad X_{5}=\left(\begin{array}{c}
\chi^{++} \\
\frac{1}{\sqrt{2}}\left(\chi^{+}-\xi^{+}\right) \\
\sqrt{\frac{2}{3}}\left(\xi^{0}-\operatorname{Re} \chi^{0}\right) \\
-\frac{1}{\sqrt{2}}\left(\chi^{+*}-\xi^{+*}\right) \\
\chi^{++*}
\end{array}\right)
$$

In terms of the custodial symmetry eigenstates, we have

$$
\operatorname{Tr}\left(X^{\dagger} X\right)=\left(X_{1}\right)^{2}+\left|X_{3}\right|^{2}+\left|X_{5}\right|^{2}
$$

where $\left|X_{3}\right|^{2} \equiv X_{3}^{\dagger} X_{3}$ and $\left|X_{5}\right|^{2} \equiv X_{5}^{\dagger} X_{5}$, and

$$
\omega=\frac{1}{4} \frac{2\left(X_{1}\right)^{2}+\left|X_{3}\right|^{2}-\left|X_{5}\right|^{2}}{\left(X_{1}\right)^{2}+\left|X_{3}\right|^{2}+\left|X_{5}\right|^{2}} .
$$

From this form it can be easily seen that $\omega$ will have a maximum value of $1 / 2$ when $X_{3}=X_{5}=0$, and a minimum value of $-1 / 4$ when $X_{1}=X_{3}=0$, yielding the total

range of $\omega \in\left[-\frac{1}{4}, \frac{1}{2}\right]$. By substituting $\left\langle\chi^{0}\right\rangle=\left\langle\xi^{0}\right\rangle=v_{\chi}$ into Eq. (7.75) and setting all other fields to zero, one will note that the desired electroweak symmetry breaking vacuum corresponds to $\omega=1 / 2$.

The maximal ranges of $r, \gamma, \zeta$ and $\omega$ have also been verified by implementing a numerical scan over all allowed field values. These ranges are tabulated in Table 7.2 along with the values corresponding to the desired electroweak vacuum.

\section{Bounds on the parameters of the scalar potential}

However, one will note from Eqs. (7.71), (7.72), and (7.75) that the variables $\zeta$ and $\omega$ are not independent of each other. The region in the $(\zeta, \omega)$ plane populated by 


\begin{tabular}{|c|c|c|c|c|c|c|}
\hline Value & $r$ & $\gamma$ & $\zeta$ & $\omega$ & $\sigma$ & $\rho$ \\
\hline \hline Maximum & $\infty$ & $\pi / 2$ & 1 & $1 / 2$ & $\sqrt{3} / 4$ & $2 / \sqrt{3}$ \\
\hline Minimum & 0 & 0 & $1 / 3$ & $-1 / 4$ & $-\sqrt{3} / 4$ & $-2 / \sqrt{3}$ \\
\hline$\left\langle\chi^{0}\right\rangle=\left\langle\xi^{0}\right\rangle=v_{\chi}$ & $\mathrm{n} / \mathrm{a}$ & $\mathrm{n} / \mathrm{a}$ & $1 / 3$ & $1 / 2$ & $\pm \sqrt{3} / 4$ & $\pm 2 / \sqrt{3}$ \\
\hline \hline
\end{tabular}

Table 7.2: Maximal ranges allowed to the variables used to parametrize the scalar potential in Sec. 7.2.2-7.2.3. The values corresponding to the desired vacuum $\left\langle\chi^{0}\right\rangle=$ $\left\langle\xi^{0}\right\rangle=v_{\chi}$ are also included; please note that the negative values allowed to $\sigma$ and $\rho$ correspond to a switch in the sign of $v_{\chi}$.

taking all possible combinations of field values is shown in Fig. 7.2. In order to bound the scalar potential parameters $\lambda_{i}(1 \leq i \leq 5)$ using Eq. (7.70), we will need to know how the maximal values of $\omega \in\left[\omega_{-}, \omega_{+}\right]$change for a given value of $\zeta$.

As indicated above, the maximum and minimum values of $\omega$ occur where only $X_{1}$ or $X_{5}$ are respectively non-zero. Considering the definitions of $X_{1,5}$ in Eq. (7.76), one will note that these values can be achieved when only $\operatorname{Re} \chi^{0}$ and $\xi^{0}$ are nonzero; we can trace the curved boundary of Fig. 7.2 while keeping only these fields in $\zeta$ and $\omega$. In this limit, $\omega$ - as defined by Eq. (7.75) — becomes

$$
\omega_{ \pm}\left(\operatorname{Re} \chi^{0}, \xi^{0}\right)=\frac{1}{2 \operatorname{Tr}\left(X^{\dagger} X\right)}\left[\left(\operatorname{Re} \chi^{0}\right)^{2}+2 \xi^{0} \operatorname{Re} \chi^{0}\right]
$$

where here $\operatorname{Tr}\left(X^{\dagger} X\right)=2\left(\operatorname{Re} \chi^{0}\right)^{2}+\left(\xi^{0}\right)^{2}$. This allows us to rewrite $\xi^{0}$ in terms of the normalization factor as $\xi^{0}= \pm \sqrt{\operatorname{Tr}\left(X^{\dagger} X\right)-2\left(\operatorname{Re} \chi^{0}\right)^{2}}$, and $\omega_{ \pm}$becomes

$$
\omega_{ \pm}(b)=\frac{b^{2}}{2} \pm 2 \sqrt{b^{2}\left(1-b^{2}\right)} \quad \text { where } \quad b=\frac{\operatorname{Re} \chi^{0}}{\sqrt{\operatorname{Tr}\left(X^{\dagger} X\right)}}
$$

We may now perform a similar transformation in Eq. (7.71) to express our variable $b$ 
in terms of $\zeta$. Starting from

$$
\zeta_{ \pm}\left(\operatorname{Re} \chi^{0}, \xi^{0}\right)=\frac{1}{\operatorname{Tr}\left(X^{\dagger} X\right)}\left[2\left(\operatorname{Re} \chi^{0}\right)^{4}+\left(\xi^{0}\right)^{4}\right]
$$

we can show that

$$
\zeta_{ \pm}(b)=6 b^{4}-4 b^{2}+1
$$

where $b$ is defined as above. Solving for $b$, we find that

$$
b_{ \pm}=\frac{1}{3}\left[1 \pm \sqrt{\frac{3}{2}\left(\zeta-\frac{1}{3}\right)}\right]
$$

We keep only $b_{-}$, as $b_{+}>1$ for certain values of $\zeta \in\left[\frac{1}{3}, 1\right]$, which is unacceptable as $b$ is defined as a normalized field. Substituting Eq. (7.83) into Eq. (7.80), we find that the upper and lower bounds on $\omega$ are given by

$$
\omega_{ \pm}(\zeta)=\frac{1}{6}(1-B) \pm \frac{\sqrt{2}}{3}\left[(1-B)\left(\frac{1}{2}+B\right)\right]^{1 / 2}
$$

with $B=1-3 b$,

$$
B \equiv \sqrt{\frac{3}{2}\left(\zeta-\frac{1}{3}\right)} \in[0,1]
$$

Using this relation and the upper and lower bounds of $\zeta \in\left[\frac{1}{3}, 1\right]$, the BFB constraints of Eq. (7.70) can be written as: ${ }^{6}$

$$
\begin{aligned}
& \lambda_{1}>0 \\
& \lambda_{4}>\left\{\begin{array}{l}
-\frac{1}{3} \lambda_{3} \text { for } \lambda_{3} \geq 0 \\
-\lambda_{3} \text { for } \lambda_{3}<0,
\end{array}\right. \\
& \lambda_{2}>\left\{\begin{array}{l}
\frac{1}{2} \lambda_{5}-2 \sqrt{\lambda_{1}\left(\frac{1}{3} \lambda_{3}+\lambda_{4}\right)} \quad \text { for } \lambda_{5} \geq 0 \text { and } \lambda_{3} \geq 0 \\
\omega_{+}(\zeta) \lambda_{5}-2 \sqrt{\lambda_{1}\left(\zeta \lambda_{3}+\lambda_{4}\right)} \text { for } \lambda_{5} \geq 0 \text { and } \lambda_{3}<0, \\
\omega_{-}(\zeta) \lambda_{5}-2 \sqrt{\lambda_{1}\left(\zeta \lambda_{3}+\lambda_{4}\right)} \text { for } \lambda_{5}<0
\end{array}\right.
\end{aligned}
$$

\footnotetext{
${ }^{6}$ Our BFB constraints are stronger than those of Ref. [144]. This is because we allow any number of the scalar fields to be non-zero, while they accounted only for combinations where two fields were non-zero.
} 


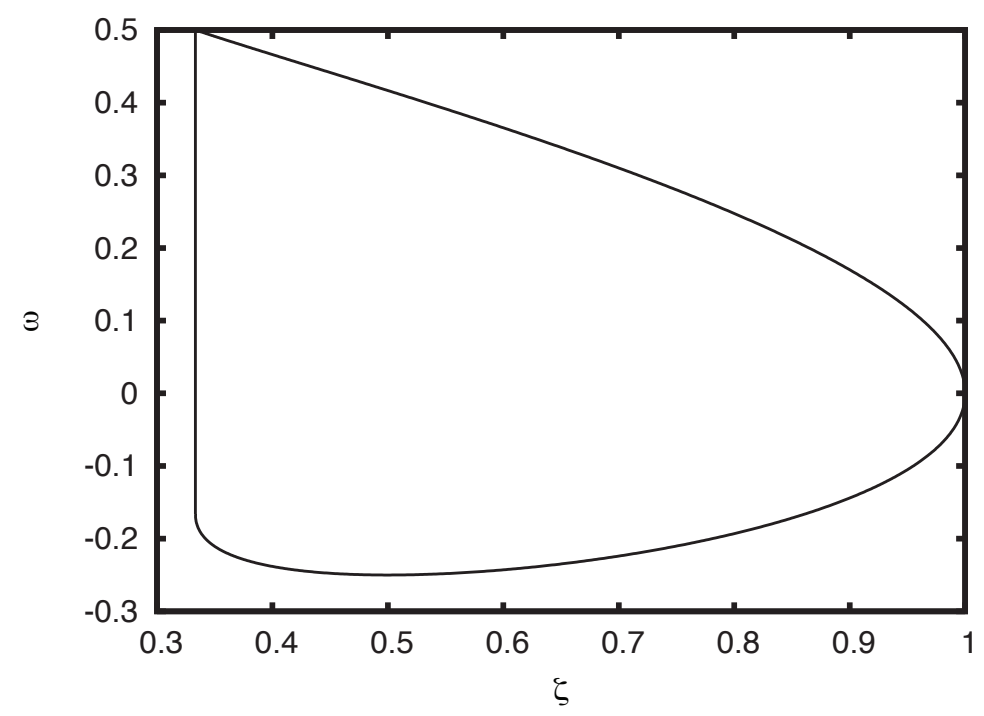

Figure 7.2: The boundary of the region in the $(\zeta, \omega)$ plane that is populated by taking all possible combinations of field values. The region enclosed by the curve is populated.

The last two conditions for $\lambda_{2}$ must be satisfied for all values of $\zeta \in\left[\frac{1}{3}, 1\right]$. These constraints reduce the allowed ranges of the scalar quartic couplings compared to those obtained from perturbative unitarity constraints in Eqs. (7.59-7.63). The boundedfrom-below constraint on $\lambda_{1}$ restricts its range to positive values,

$$
\lambda_{1} \in\left(0, \frac{1}{3} \pi\right) \simeq(0,1.05)
$$

As in the case of the unitarity bounds, the BFB constraints limit a linear combination of $\lambda_{3}$ and $\lambda_{4}$, corresponding to two new lines in the $\left(\lambda_{3}, \lambda_{4}\right)$ plane, as shown in Fig. 7.3. In this figure, the BFB constraint excludes the regions below the dot-dashed lines, while the unitarity constraint from $\left|x_{1}^{ \pm}\right|<8 \pi$ restricts $\lambda_{3}$ and $\lambda_{4}$ to lie between the two solid lines (we again set $\lambda_{2}=0$ for the least restrictive constraint on $\lambda_{3}$ and $\lambda_{4}$ from $\left.x_{1}^{ \pm}\right)$. The allowed region is a triangle with vertices at $\left(\lambda_{3} / \pi, \lambda_{4} / \pi\right)=(0,0),\left(-\frac{1}{2}, \frac{1}{2}\right)$, and $\left(\frac{3}{5},-\frac{1}{5}\right)$. The unitarity constraint from $y_{1}$ becomes 


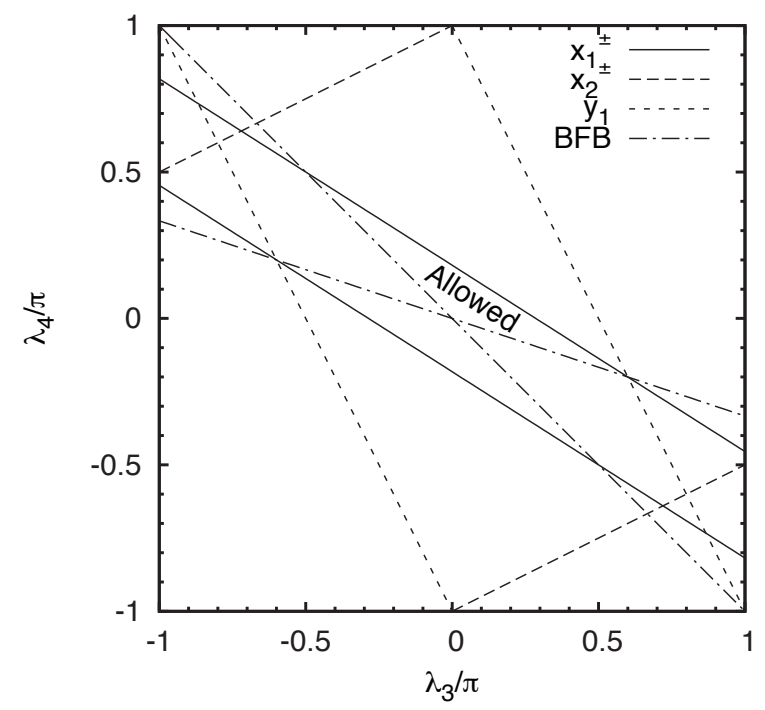

Figure 7.3: Constraints on the $\left(\lambda_{3}, \lambda_{4}\right)$ plane from perturbative unitarity, as in Fig. 3.1, together with the bounded-from-below (BFB) constraints $\lambda_{4}>-\frac{1}{3} \lambda_{3}$ and $\lambda_{4}>-\lambda_{3}$.

superfluous; however, the unitarity constraint from $x_{2}^{ \pm}$can still be important for large enough values of $\left|\lambda_{5}\right|$. As a result, the maximum accessible ranges of $\lambda_{3}$ and $\lambda_{4}$ are reduced compared to those given in Eq. (7.61) to read

$$
\begin{aligned}
& \lambda_{3} \in\left(-\frac{1}{2} \pi, \frac{3}{5} \pi\right) \simeq(-1.57,1.88), \\
& \lambda_{4} \in\left(-\frac{1}{5} \pi, \frac{1}{2} \pi\right) \simeq(-0.628,1.57) .
\end{aligned}
$$

Finally, the BFB constraint restricts the accessible range of $\lambda_{2}$ as follows. The least restrictive lower bound on $\lambda_{2}$ from unitarity is obtained by taking $\lambda_{1}=0$ and $7 \lambda_{3}+11 \lambda_{4}=0$ in $x_{1}^{ \pm}$. However, when $\lambda_{1}=0$, the BFB constraint on $\lambda_{2}$ forces $\lambda_{2}>0$, with the least restrictive constraint obtained for $\lambda_{5}=0$. The least restrictive lower bound on $\lambda_{2}$ will therefore occur for nonzero values of $\lambda_{1}, \lambda_{3}$ and $\lambda_{4}$ and can be obtained through a numerical scan.

The maximal allowed ranges on all parameters $\lambda_{i}$ and $M_{i}$ of the scalar potential 


\begin{tabular}{|c|c|c|c|c|c|c|c|}
\hline Parameter & $\lambda_{1}$ & $\lambda_{2}$ & $\lambda_{3}$ & $\lambda_{4}$ & $\lambda_{5}$ & $M_{1} / \sqrt{\mu_{3}^{2}}$ & $M_{2} / \sqrt{\mu_{3}^{2}}$ \\
\hline \hline Maximum & 1.05 & 2.09 & 1.88 & 1.57 & 8.38 & 3.3 & 1.2 \\
Minimum & 0 & -2.09 & -1.57 & -0.628 & -8.38 & -3.3 & -1.2 \\
\hline \hline
\end{tabular}

Table 7.3: Theoretical bounds on the scalar potential parameters $\lambda_{i}$ and $M_{i}$ from $8 v_{\chi}^{2} \leq v^{2}$, perturbative unitarity, and bounded-from-below constraints. Constraints from avoiding alternative vacua are not included.

Eq. (7.10) from unitarity and BFB constraints are summarized in Table 7.3. The tabulated bound on $M_{2}$ arises from the requirement that $8 v_{\chi}^{2} \leq v^{2}$, and is further discussed at the end of Sec. 7.2.3.

\subsubsection{Avoiding alternative vacua}

We now consider the conditions on the parameters of the scalar potential that are required in order to ensure that the desired EWSB and custodial SU(2)-preserving minimum is the true global minimum. As described in Sec. 3.1.3, for a given set of potential parameters $\lambda_{i}(1 \leq i \leq 5)$ we must scan over the scalar field values in order to check whether the potential minimum corresponding to the electroweak vacuum is lower than any alternative minimum.

To do this, it is helpful to parametrize the full scalar potential in Eq. (7.10) using the variables $r, \gamma, \zeta$, and $\omega$ defined in Eq. (7.64-7.68) of the previous section, as well as two new variables,

$$
\begin{gathered}
\sigma \equiv \frac{\operatorname{Tr}\left(\Phi^{\dagger} \tau^{a} \Phi \tau^{b}\right)\left(U X U^{\dagger}\right)_{a b}}{\operatorname{Tr}\left(\Phi^{\dagger} \Phi\right)\left[\operatorname{Tr}\left(X^{\dagger} X\right)\right]^{1 / 2}} \\
\rho \equiv \frac{\operatorname{Tr}\left(X^{\dagger} t^{a} X t^{b}\right)\left(U X U^{\dagger}\right)_{a b}}{\left[\operatorname{Tr}\left(X^{\dagger} X\right)\right]^{3 / 2}}
\end{gathered}
$$


These new variables may be explicitly written as

$$
\begin{aligned}
\sigma & =\frac{1}{4} \frac{\left(2 \operatorname{Re} \chi^{0}+\xi^{0}\right)}{\left[\operatorname{Tr}\left(X^{\dagger} X\right)\right]^{1 / 2}} \\
\rho & =\frac{6}{\left[\operatorname{Tr}\left(X^{\dagger} X\right)\right]^{3 / 2}}\left\{\xi^{0}\left(\left|\chi^{0}\right|^{2}-\left|\chi^{++}\right|^{2}\right)+2 \operatorname{Re}\left[\xi^{+}\left(\chi^{0} \chi^{+*}+\chi^{+} \chi^{++*}\right)\right]\right\} .
\end{aligned}
$$

where we have made use of Eq. (7.74) in $\sigma$, and $\operatorname{Tr}\left(X^{\dagger} X\right)$ is defined explicitly in Eq. (7.72). The allowed ranges of these two new parameters are $\sigma \in\left[-\frac{\sqrt{3}}{4}, \frac{\sqrt{3}}{4}\right]$ and $\rho \in\left[-\frac{2}{\sqrt{3}}, \frac{2}{\sqrt{3}}\right]$; these may be found either from analytical arguments very similar to those given for $\zeta$ and $\omega$ in the previous section, or using a numerical scan [4]. By substituting $\left\langle\chi^{0}\right\rangle=\left\langle\xi^{0}\right\rangle=v_{\chi}$ into Eqs. (7.91-7.92) and setting all other fields to zero, one will note that the desired electroweak symmetry breaking vacuum corresponds to respective values of $\sigma=\sqrt{3} / 4$ and $\rho=2 / \sqrt{3} .7$

The potential may then be written as follows,

$$
\begin{aligned}
V= & \frac{r^{2}}{\left(1+\tan ^{2} \gamma\right)} \frac{1}{2}\left[\mu_{2}^{2}+\mu_{3}^{2} \tan ^{2} \gamma\right]+\frac{r^{3}}{\left(1+\tan ^{2} \gamma\right)^{3 / 2}} \tan \gamma\left[-\sigma M_{1}-\rho M_{2} \tan ^{2} \gamma\right] \\
& +\frac{r^{4}}{\left(1+\tan ^{2} \gamma\right)^{2}}\left[\lambda_{1}+\left(\lambda_{2}-\omega \lambda_{5}\right) \tan ^{2} \gamma+\left(\zeta \lambda_{3}+\lambda_{4}\right) \tan ^{4} \gamma\right] .
\end{aligned}
$$

In order to check that the desired EWSB vacuum is the global minimum of the scalar potential, we use the values of $\zeta, \omega, \sigma$ and $\rho$ that correspond to our desired vacuum to determine the values of $r$ and $\tan \gamma$ corresponding to a minimum of Eq. (7.93). We then compute the corresponding minimum value of of the potential $\left(V_{\mathrm{min}}^{\mathrm{EW}}\right)$, and check that it is lower than any alternative field configuration $\left(V_{\min }^{\mathrm{EW}}<V_{\min }^{\text {alt }}\right)$ by repeating the process for every possible combination of values for $\zeta, \omega, \sigma$, and $\rho$.

An analytic method for simplifying this eight-dimensional parameter scan to a one-dimensional scan is outlined in Ref. [4]. We find that when $\lambda_{3}, \lambda_{5}, M_{1}$, and $M_{2}$ are all positive, the desired vacuum is always the true global minimum of the

\footnotetext{
${ }^{7}$ The vacuum with $\sigma=-\sqrt{3} / 4$ and $\rho=-2 / \sqrt{3}$ is also acceptable; it corresponds to negative $v_{\chi}$.
} 
potential. Simultaneously flipping the signs of $M_{1}, M_{2}$, and $X$ leaves the scalar potential invariant, so the alternative acceptable vacuum that corresponds to $v_{\chi} \rightarrow$ $-v_{\chi}$ is always the true global minimum when $\lambda_{3}$ and $\lambda_{5}$ are positive and $M_{1}$ and $M_{2}$ are both negative. For all other sign combinations of these four parameters, the depth of alternative minima must be checked numerically.

Note that even when the desired vacuum corresponds to the global minimum of the potential, one must still verify that the potential is minimized for an acceptable vacuum expectation value $v_{\chi}$, as defined by Eq. (7.15). Due to the relation $8 v_{\chi}^{2}+v_{\phi}^{2}=$ $v^{2}, v_{\chi}$ is required to be smaller than the SM vev, $8 v_{\chi}^{2} \leq v^{2}$. However, for certain combinations of parameters, the lowest minimum in the potential will correspond to a triplet vev that is too large, $8 v_{\chi}^{2}>v^{2}$; this indicates that the doublet vev $v_{\phi}$ has become complex, and that the model is no longer in a minimum of the scalar potential in the $\left(v_{\phi}, v_{\chi}\right)$ plane. This occurs when the dimensionful parameter $M_{2}$ becomes too large in comparison with $\sqrt{\mu_{3}^{2}}$; as a result — similarly to the $M_{1}$ case - the parameter $M_{2}$ may only scale linearly with $\mu_{3}$, and must satisfy a rough numerical constraint of $\left|M_{2}\right| / \sqrt{\mu_{3}^{2}} \lesssim 1.2$. This bound is tabulated with the other maximal parameter limits in Table 7.3.

\subsection{The decoupling limit}

When investigating the phenomenology of a BSM model, it is often useful to consider the existence and behaviour of a decoupling limit. A model has a decoupling limit if its parameter space allows for all of the new BSM particles to simultaneously become very heavy, leaving only the SM field content at the weak scale. In this scenario, the BSM physics effects will become very small as the masses become very large, causing 
the BSM physics to decouple from the low-energy states. The remaining low-energy theory is generally required to be very SM-like in order to remain consistent with experimental data. As such, many models reduce to the SM in their decoupling limit, and therefore cannot be fully excluded by experiment if the SM remains viable.

As briefly discussed in Sec. 7.1, historically much of the work on the GM model has made use of a $Z_{2}$-symmetric potential that set $M_{1}=M_{2}=0$ in Eq. (7.10). The resulting model did not have a decoupling limit. The scalar potential contained only two dimensionful parameters $\mu_{2}$ and $\mu_{3}$ which could set the mass scale, and both of these parameters were eliminated in favour of the vacuum expectation values using the minimization conditions. The masses of the new BSM scalars could therefore only depend on dimensionless parameters $\lambda_{i}$ and the vevs $v_{\phi} \leq v$ and $v_{\chi} \leq v / \sqrt{8}$, resulting in a relationship of form $m_{s}^{2} \propto \lambda_{i} v^{2}$. As a result, the unitarity bounds on the parameters $\lambda_{i}$ constrained the BSM scalar masses to be less than about $700 \mathrm{GeV}[5]$. Since the masses could not be made arbitrarily heavy, the model could not decouple.

However, the presence of $M_{1}$ and $M_{2}$ in Eq. (7.10) allows for a decoupling limit in the more general form of the model. In this case the mass scale of the new particles is set by the unbounded dimensionful parameter $\mu_{3}$, as can be seen by considering Eqs. (7.24-7.26) and (7.35). We will therefore consider the behaviour of the scalar mass spectrum, the vevs, the custodial-singlet mixing angle, and the couplings of the light Higgs to SM particles as $\mu_{3}$ becomes very large $\left(\mu_{3} \gg \lambda_{i} v\right)$. In the following we derive explicit expansions for each of these observables in this limit, keeping terms up to next-to-leading order in inverse powers of $\mu_{3}^{2}$. As we will show, all of the low-energy observables reduce to their appropriate SM limits as $\mu_{3} \rightarrow \infty$.

In these expansions we consider two different cases for the behaviour of $M_{1}$ and $M_{2}$. Being dimensionful, these parameters are permitted to vary with powers 


\begin{tabular}{ccccccccc}
\hline \hline Case & $\mu_{3} \equiv \sqrt{\left|\mu_{3}^{2}\right|}$ & $\lambda_{1}$ & $\lambda_{2}$ & $\lambda_{3}$ & $\lambda_{4}$ & $\lambda_{5}$ & $M_{1}$ & $M_{2}$ \\
\hline $\mathrm{A}$ & $300-1000 \mathrm{GeV}$ & derived & 0.1 & 0.1 & 0.1 & 0.1 & $100 \mathrm{GeV}$ & $100 \mathrm{GeV}$ \\
$\mathrm{B}$ & $300-1000 \mathrm{GeV}$ & derived & 0.1 & 0.1 & 0.1 & 0.1 & $\mu_{3} / 3$ & $\mu_{3} / 3$ \\
\hline \hline
\end{tabular}

Table 7.4: Values of coupling parameters for the two decoupling scenarios considered. We use $m_{h}=125 \mathrm{GeV}$ to fix $\lambda_{1}$ in terms of the other parameters, while $\mu_{2}^{2}$ is eliminated in terms of the known SM Higgs vev $v$.

of $\mu_{3}$, and could in theory take the form $M_{1,2} \propto \mu_{3}^{m} / v^{m-1}$. However, the bounds on these parameters from theoretical constraints require that $M_{1,2}$ vary at most linearly with $\mu_{3}(m \leq 1)$, as discussed in Sec. 7.2.1 and 7.2.3 (see Table 7.3). We will therefore consider two possibilities in our decoupling expansions: $M_{1,2}$ may be held constant as $\mu_{3}$ becomes large (Case A), or $M_{1,2}$ could vary linearly with $\mu_{3}$ as $\mu_{3}$ increases (Case B). In order to preserve next-to-leading order terms in our expansion formulas in the Case B scenario, we will treat factors of $M_{i} / \mu_{3}$ as though they are constant when eliminating higher order terms. The decoupling formulas presented below are therefore valid approximations regardless of how $M_{1,2}$ vary with $\mu_{3}$.

In order to graphically compare the behaviour of these two scenarios in the decoupling limit, we will also make use of a benchmark parameter set for each case. These benchmark points are chosen to satisfy all of the theoretical constraints discussed in Sec. 7.2, and are tabulated in Table 7.4. Note that in these cases either both $M_{1,2}$ are held constant, or both scale with $\mu_{3}$. This is because the overall decoupling behaviour is much more strongly influenced by $M_{1}$ than by $M_{2}$; as we will show, a scenario with $M_{1} \propto \mu_{3}$ and $M_{2}$ constant will closely mimic Case B, while a scenario with $M_{1}$ constant and $M_{2} \propto \mu_{3}$ will resemble Case A.

The overall power law dependence of each observable on $\mu_{3}$ in the decoupling 


\begin{tabular}{ccc}
\hline \hline Quantity & Case A & Case B \\
\hline$\frac{m_{H, 3,5}}{\mu_{3}}-1$ & $\mu_{3}^{-2}$ & $\mu_{3}^{-2}$ \\
$v_{\chi}$ & $\mu_{3}^{-2}$ & $\mu_{3}^{-1}$ \\
$\sin \alpha$ & $\mu_{3}^{-2}$ & $\mu_{3}^{-1}$ \\
$\kappa_{V}-1$ & $\mu_{3}^{-4}$ & $\mu_{3}^{-2}$ \\
$\kappa_{f}-1$ & $\mu_{3}^{-4}$ & $\mu_{3}^{-2}$ \\
$g_{h h V V} / g_{h h V V}^{\mathrm{SM}}-1$ & $\mu_{3}^{-4}$ & $\mu_{3}^{-2}$ \\
$g_{h h h} / g_{h h h}^{\mathrm{SM}}-1$ & $\mu_{3}^{-4}$ & $\mu_{3}^{-2}$ \\
$\Delta \kappa_{\gamma}$ & $\mu_{3}^{-2}$ & $\mu_{3}^{-2}$ \\
$\Delta \kappa_{Z \gamma}$ & $\mu_{3}^{-2}$ & $\mu_{3}^{-2}$ \\
\hline \hline
\end{tabular}

Table 7.5: The power law behavior of the heavy scalar masses, triplet vev, custodial singlet mixing angle, and light Higgs couplings for parameter cases A and B. See text for definitions.

limit is tabulated in Table 7.5. In general the convergence to the SM is faster in Case A, where the observables approach the decoupling limit at rates proportional to $\mu_{3}^{-2}$ or $\mu_{3}^{-4}$. In comparison, in Case $\mathrm{B}$ the decoupling rates are proportional to $\mu_{3}^{-1}$ or $\mu_{3}^{-2}$.

\subsubsection{The decoupling behaviour of the vevs and mixing angles}

We begin our derivation with Eq. (7.15), which defines the value of $v_{\chi}$. In the limit that $\mu_{3} \rightarrow \infty$, the cubic equation for $v_{\chi}$ reduces to a quartic, which may be Taylor expanded and rearranged to give the following formula for the triplet vev in the decoupling limit,

$$
v_{\chi} \simeq \frac{M_{1} v^{2}}{4 \mu_{3}^{2}}\left[1-\left(2 \lambda_{2}-\lambda_{5}\right) \frac{v^{2}}{\mu_{3}^{2}}+\frac{M_{1}\left(3 M_{2}-M_{1}\right) v^{2}}{2 \mu_{3}^{4}}\right]
$$



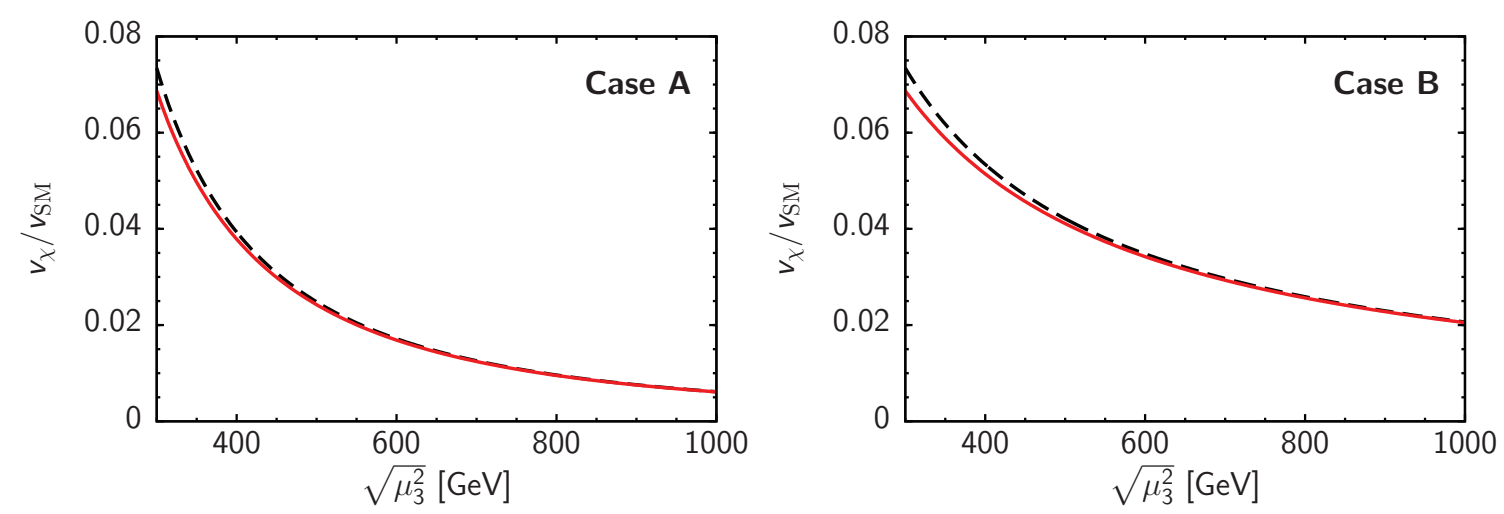

Figure 7.4: The dependence of the triplet vev $v_{\chi}$ (shown normalized to $v=246 \mathrm{GeV}$ ) as a function of $\mu_{3}$ in cases $\mathrm{A}$ (left) and $\mathrm{B}$ (right). In all plots the solid red (light) line is the exact curve, while the dashed black (dark) line is the corresponding expansion formula from Eq. (7.94).

The triplet vev approaches its SM value of zero $\left(v_{\chi} \rightarrow 0\right)$ as $\mu_{3} \rightarrow \infty$, where its value falls like $\mu_{3}^{-2}\left(\mu_{3}^{-1}\right)$ in Case A (Case B). This decoupling behaviour of $v_{\chi}$ is plotted for Cases A and B in Fig. (7.4). In this figure - as in all of the decoupling plots in this section — the solid lines illustrate the exact decoupling behaviour, while the dashed black lines illustrate the approximate decoupling formula for comparison. Using Eq. (7.94) and the relation between the scalar vevs $v_{\phi}^{2}+8 v_{\chi}^{2}=v^{2}$, the decoupling expansion for the doublet vev can similarly be found to be

$$
v_{\phi} \simeq v\left(1-\frac{M_{1}^{2} v^{2}}{4 \mu_{3}^{4}}\right)
$$

which approaches the SM value of $v$ in the decoupling limit.

As $s_{H}=2 \sqrt{2} v_{\chi} / v$ and $c_{H}=v_{\phi} / v$, the mixing angle $\theta_{H}$ will also approach zero in the decoupling limit. In this limit the Goldstone boson states become SM-like, as they no longer have a component from the triplet states $\chi$ and $\xi$ (as demonstrated by Eqs. (7.16-7.17)). Similarly, the $H_{3}$ states become independent from the doublet states $\phi$ as $s_{H} \rightarrow 0$ (as seen in Eqs. (7.22-7.23). The doublet states $\phi$ are then decoupled from 
the triplet states $\chi, \xi$, resulting in SM scalars $G^{0, \pm}$ that are completely independent of the BSM scalars $H_{3}^{0, \pm}$.

One can similarly show that the second mixing angle $\alpha$ also approaches zero, resulting in a similar decoupling of the SM Higgs state. However, $\alpha$ depends on $\lambda_{1}$, which is related to the triplet vev $v_{\chi}$ through its definition in terms of $m_{h}$ in Eq. (7.36). As a result, the parameter $\lambda_{1}$ varies with $\mu_{3}$ as

$$
\lambda_{1} \simeq \frac{1}{8}\left[\frac{m_{h}^{2}}{v^{2}}+\frac{3}{4} \frac{M_{1}^{2}}{\mu_{3}^{2}}\left(1-3\left(2 \lambda_{2}-\lambda_{5}\right) \frac{v^{2}}{\mu_{3}^{2}}+\frac{3 M_{1} M_{2} v^{2}}{\mu_{3}^{4}}+\frac{5 m_{h}^{2}}{3 \mu_{3}^{2}}\right)\right] .
$$

The first term of this formula coincides with the value of the SM quartic coupling, $\lambda_{1}=m_{h}^{2} / 8 v^{2}$. In Case $\mathrm{A} \lambda_{1}$ will approach this value as $\mu_{3} \rightarrow \infty$, but in Case B the $\mu_{3} \rightarrow \infty$ limit of $\lambda_{1}$ is $\left(m_{h}^{2} / 8 v^{2}+3 M_{1}^{2} / 32 \mu_{3}^{2}\right)$. This discrepancy arises because $\lambda_{1}$ does not correspond directly to the SM Higgs quartic coupling; the full triple-Higgs coupling (defined in Appendix A.3) decouples like

$$
C_{h h h} \simeq \frac{3 m_{h}^{2}}{v}\left\{1-\frac{M_{1}^{2} v^{2}}{\mu_{3}^{4}}\left[\frac{7}{8}-\frac{3}{2} \frac{v^{2}}{m_{h}^{2}}\left(\left(2 \lambda_{2}-\lambda_{5}\right)+\frac{M_{1} M_{2}}{\mu_{3}^{2}}\right)\right]\right\},
$$

which reduces to the SM value as $\mu_{3} \rightarrow \infty$ even in Case B. Eq. (7.96) is also the origin of the unitarity bound on $M_{1}$; one will recognize the first two terms from Eq. (7.60).

Using the decoupling expansions for $v_{\chi}$ (Eq. (7.94)) and $\lambda_{1}$ (Eq. (7.96)), the decoupling behaviour of the mixing angle $\alpha$ (defined in Eq. (7.34)) is found to be

$$
\sin \alpha \simeq-\frac{\sqrt{3} M_{1} v}{2 \mu_{3}^{2}}\left[1-2\left(2 \lambda_{2}-\lambda_{5}\right) \frac{v^{2}}{\mu_{3}^{2}}+\frac{m_{h}^{2}}{\mu_{3}^{2}}+\frac{M_{1}\left(24 M_{2}-5 M_{1}\right) v^{2}}{8 \mu_{3}^{4}}\right] .
$$

We can see that $\sin \alpha$ approaches zero as $\mu_{3} \rightarrow \infty$. This is to be expected, as $\sin \alpha$ controls the amount of triplet in the mass eigenstate $h$, and $\sin \alpha=0$ corresponds to a SM-like Higgs boson $h$ composed entirely of the $\mathrm{SU}(2)_{L}$ doublet (recall Eq. (7.28)). The rate of the decoupling is proportional to $\mu_{3}^{-2}$ in Case $\mathrm{A}$ and $\mu_{3}^{-1}$ in Case $\mathrm{B}$, as shown in Fig. 7.5. We will show that $\sin \alpha$ and $v_{\chi}$ are the only quantities that may 

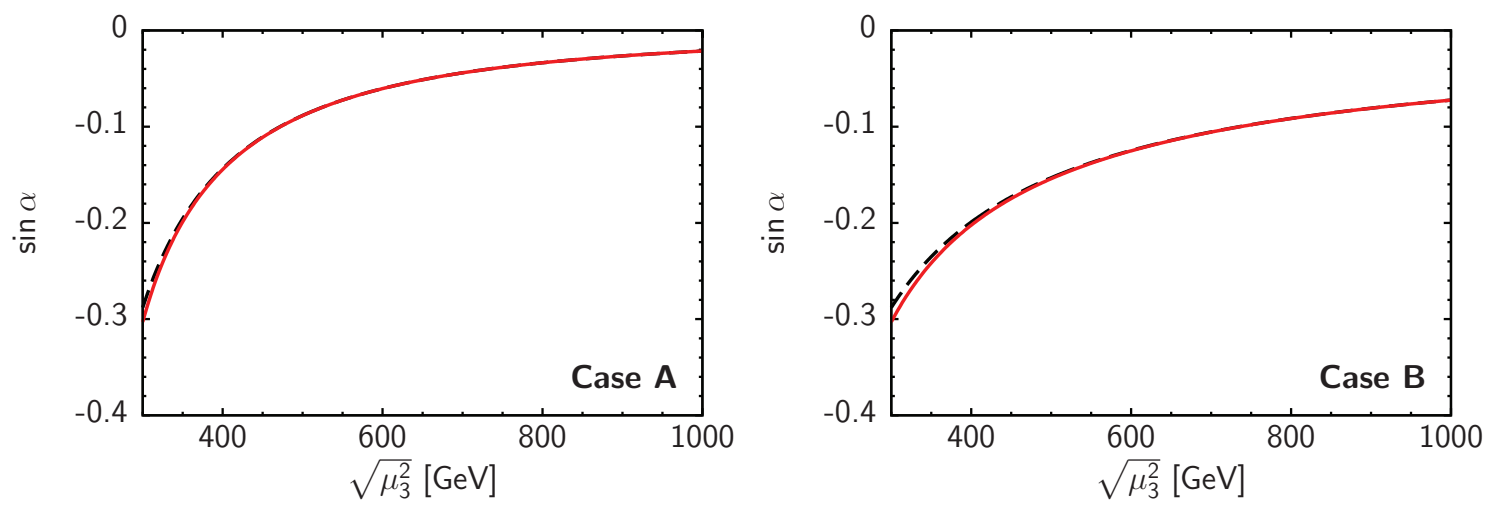

Figure 7.5: The mixing angle $\sin \alpha$ that controls the composition of the light Higgs boson $h=\phi^{0, r} \cos \alpha-H_{1}^{0 \prime} \sin \alpha$, shown as a function of $\mu_{3}$ in cases A (left) and B (right). In all plots the solid red (light) line is the exact curve, while the dashed black (dark) line is the corresponding expansion formula from Eq. (7.98).

decouple as slowly as $\mu_{3}^{-1}$; all others decouple at a rate of $\mu_{3}^{-2}$ or faster regardless of the case under consideration.

\subsubsection{The decoupling behaviour of the scalar masses}

With the decoupling formulas for the vev $v_{\chi}$ (Eq. (7.94)) and $\lambda_{1}$ (Eq. (7.96)) in hand, we may consider the behaviour of the heavy scalar masses originally defined in Eqs. (7.24), (7.25) and (7.35). These masses decouple according to

$$
\begin{aligned}
& m_{3} \simeq \mu_{3}\left[1+\left(2 \lambda_{2}-\frac{\lambda_{5}}{2}\right) \frac{v^{2}}{2 \mu_{3}^{2}}+\frac{M_{1}\left(M_{1}-3 M_{2}\right) v^{2}}{4 \mu_{3}^{4}}\right], \\
& m_{5} \simeq \mu_{3}\left[1+\left(2 \lambda_{2}+\frac{\lambda_{5}}{2}\right) \frac{v^{2}}{2 \mu_{3}^{2}}+\frac{3 M_{1} M_{2} v^{2}}{4 \mu_{3}^{4}}\right], \\
& m_{H} \simeq \mu_{3}\left[1+\left(2 \lambda_{2}-\lambda_{5}\right) \frac{v^{2}}{2 \mu_{3}^{2}}+\frac{3 M_{1}\left(M_{1}-4 M_{2}\right) v^{2}}{8 \mu_{3}^{4}}\right] .
\end{aligned}
$$

As expected, the BSM scalar masses scale with $\mu_{3}$, indicating that $\mu_{3}$ sets the BSM mass scale. The fractional difference $\left(m_{3,5, H}-\mu_{3}\right) / \mu_{3}$ scales with $\mu_{3}^{-2}$ in both Case A and Case B, as shown in Fig. 7.6. The overall decoupling behavior is similar in both 

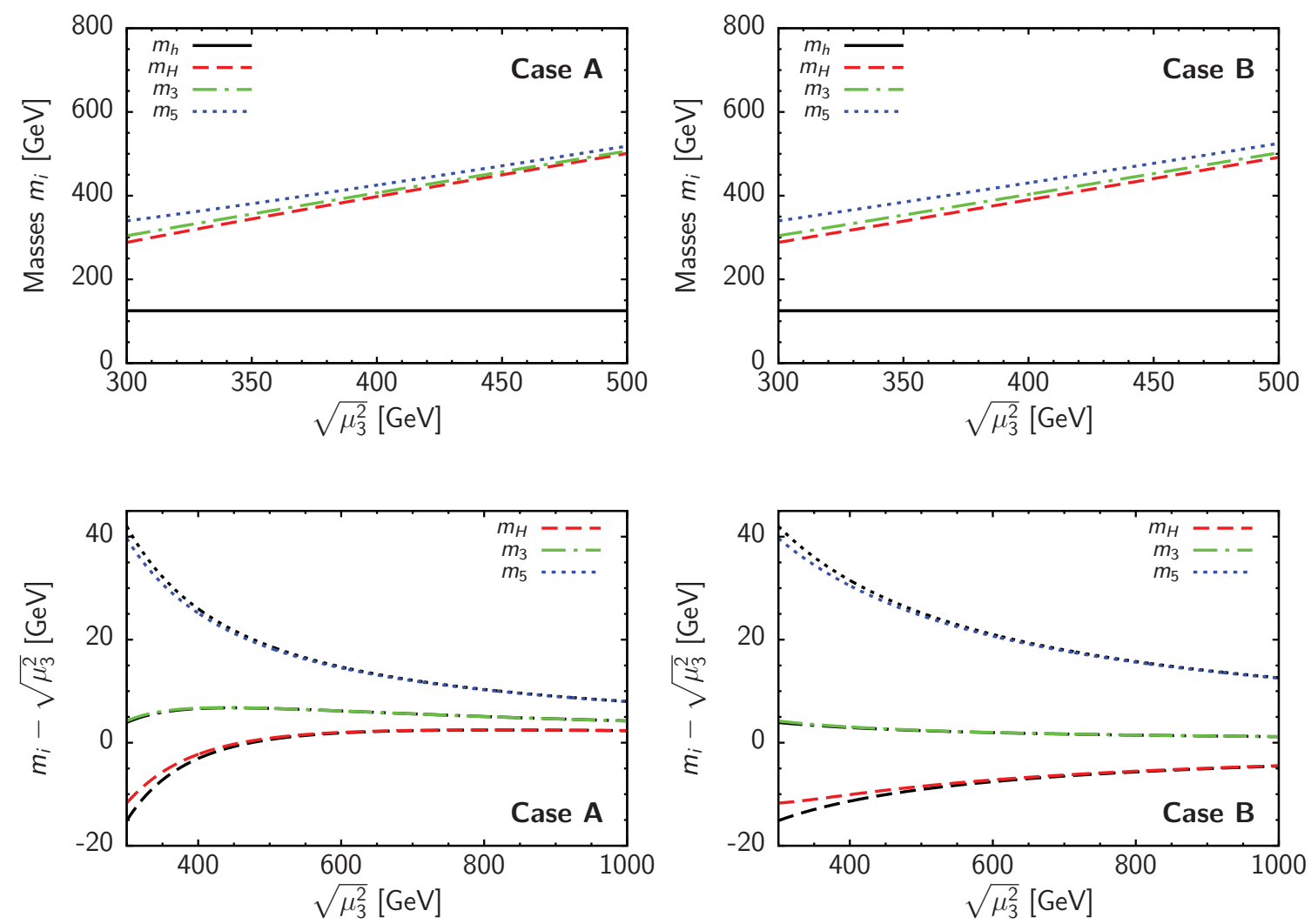

Figure 7.6: Top: The mass spectrum of the model as a function of $\sqrt{\mu_{3}^{2}}$ for cases $\mathrm{A}$ (left) and B (right). Bottom: The mass splittings $m_{i}-\sqrt{\mu_{3}^{2}}$ for the heavy scalars as a function of $\mu_{3}$ for cases $\mathrm{A}$ (left) and $\mathrm{B}$ (right). In the bottom plots the colored (light) curves show the exact tree-level masses while the black (dark) curves are the associated expansion formulas from Eqs. (7.99)-(7.101).

cases; the primary difference is the size of the mass splittings, which are larger in Case B due to the increased numerical size of the terms involving $M_{1}$ and $M_{2}$.

\subsubsection{The decoupling of tree-level Higgs couplings}

We now consider the decoupling behavior of the tree-level couplings of the light Higgs boson $h$ to SM fermions and vector bosons. As defined in Eq. (3.64), the couplings of a single Higgs to SM fermions $\left(C_{h \bar{f} f}\right)$ and gauge bosons $\left(C_{h V V}\right)$ may be written 

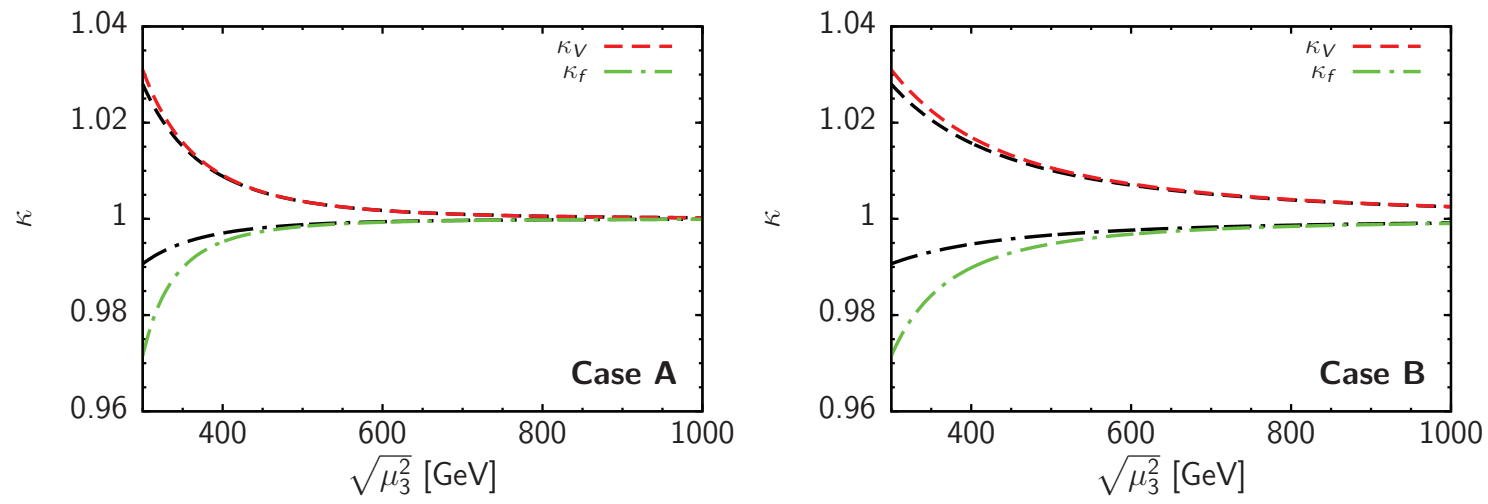

Figure 7.7: The light Higgs coupling modification factors $\kappa_{V}$ (upper dashed curves) and $\kappa_{f}$ (lower dot-dashed curves) as a function of $\mu_{3}$, for cases A (left) and B (right). The colored (light) curves are the exact results while the black (dark) curves show the corresponding expansion formulas as in Eqs. (7.102) and (7.103).

as ratios $\kappa_{f, V}$ to the corresponding SM couplings. Using the results from Eq. (7.94), (7.95) and (7.98), we expand these ratios and the quartic $C_{h h V V}$ coupling in the decoupling limit, obtaining

$$
\begin{aligned}
\kappa_{V} & =\cos \alpha \frac{v_{\phi}}{v}-\frac{8}{\sqrt{3}} \sin \alpha \frac{v_{\chi}}{v} \simeq 1+\frac{3}{8} \frac{M_{1}^{2} v^{2}}{\mu_{3}^{4}} \\
\kappa_{f} & =\cos \alpha \frac{v}{v_{\phi}} \simeq 1-\frac{1}{8} \frac{M_{1}^{2} v^{2}}{\mu_{3}^{4}} \\
C_{h h V V} & =\frac{2 m_{V}^{2}}{v^{2}}\left(\cos ^{2} \alpha+\frac{8}{3} \sin ^{2} \alpha\right) \simeq \frac{2 m_{V}^{2}}{v^{2}}\left(1+\frac{5}{4} \frac{M_{1}^{2} v^{2}}{\mu_{3}^{4}}\right),
\end{aligned}
$$

As illustrated in Fig. 7.7, both $\kappa_{f}$ and $\kappa_{V}$ approach the SM value of 1 as $\mu_{3} \rightarrow \infty$ at a rate proportional to $\mu_{3}^{-4}\left(\mu_{3}^{-2}\right)$ in Case A (Case B). The expansion formula for $\kappa_{f}$ is only accurate for $\mu_{3} \geq 400 \mathrm{GeV}$; below this scale subleading terms become important, as shown by the deviation between the exact and approximate curves in Fig. 7.7.

One will note from Eq. (7.102) that $\kappa_{V}$ will always approach the SM limit from above for large $\mu_{3}$, as the next-to-leading-order term in the expansion will always be positive. Similarly, $\kappa_{f}$ will always approach the SM value from below. In other 

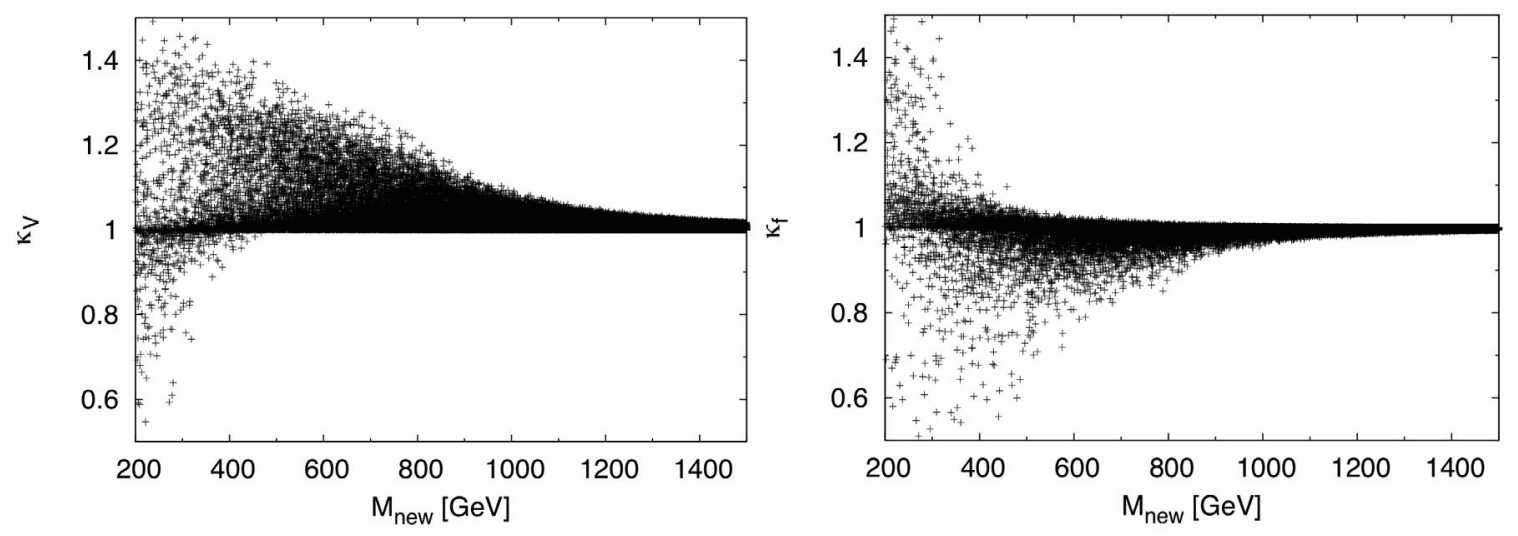

Figure 7.8: The light Higgs coupling modification factors $\kappa_{V}$ (upper) and $\kappa_{f}$ (lower) as a function of the mass of the lightest of the new scalars, $M_{\text {new }}=\min \left(m_{H}, m_{3}, m_{5}\right)$. The right panels shows a close-up of the region of small coupling deviations.

words, when the mass scale of the new scalar states is high, Higgs couplings to SM vector bosons will be enhanced compared to the SM, while couplings to fermions will be suppressed. The Higgs couplings to SM particles therefore cannot all be simultaneously enhanced — as discussed at the beginning of this chapter — if the new GM scalars are heavier than about $500 \mathrm{GeV}$. However, subleading terms neglected in Eqs. (7.102) and (7.103) become important for $\mu_{3} \leq 500 \mathrm{GeV}$ and can lead to $\kappa_{V}$ and $\kappa_{f}$ values either larger or smaller than in the SM.

This decoupling behaviour of the Higgs couplings is illustrated in Fig. 7.8. In these figures we have scanned over the parameter space allowed by the theoretical constraints discussed in Sec. 7.2, and plotted the resulting $\kappa_{f, V}$ as a function of the lightest BSM scalar mass. Note that the theory constraints allow for both $\kappa_{V}$ and $\kappa_{f}$ to be larger than the SM value of 1 when the lightest new scalar is below $500 \mathrm{GeV}$. However, above this mass scale $\kappa_{f}$ are suppressed, $\kappa_{V}$ are enhanced, and both ratios approach 1 in the high-mass limit as expected.

Additionally, the decoupling behaviour of $\kappa_{V}$ suggests that precision measure- 


\begin{tabular}{cccc}
\hline Model & GM A & GM B & 2HDM \\
\hline$\kappa_{V}$ & $\mu_{3}^{-4}$ & $\mu_{3}^{-2}$ & $m_{A}^{-4}$ \\
$\kappa_{f}$ & $\mu_{3}^{-4}$ & $\mu_{3}^{-2}$ & $m_{A}^{-2}$ \\
$C_{h h h}^{2 \mathrm{HDM}} / g_{h h h}^{\mathrm{SM}}$ & $\mu_{3}^{-4}$ & $\mu_{3}^{-2}$ & $m_{A}^{-2}$ \\
\hline
\end{tabular}

Table 7.6: Decoupling rate of the $H$ couplings in the GM and 2HDM in terms of their respective new physics mass scales $\mu_{3}$ and $m_{A}$.

ments of the Higgs couplings could differentiate the GM model from models involving scalar doublets. For example, the Two Higgs Doublet Model (2HDM) has a scalar spectrum similar to that of the GM model: two CP-even neutral scalars $h^{0}$ and $H^{0}$, a CP-odd neutral scalar $A^{0}$, and charged scalars $H^{ \pm}$. The decoupling behaviour of $\kappa_{f}$ and $\kappa_{V}$ in this model is given by [149]

$$
\begin{array}{r}
\kappa_{V}^{2 \mathrm{HDM}}-1 \propto-\frac{v^{4}}{2 m_{A}^{4}}, \\
\kappa_{f}^{2 \mathrm{HDM}}-1 \propto-\frac{v^{2}}{m_{A}^{2}}, \\
g_{h h h}^{2 \mathrm{HDM}} / g_{h h h}^{\mathrm{SM}}-1 \propto-\frac{v^{2}}{\lambda m_{A}^{2}},
\end{array}
$$

where the mass scale $m_{A}$ is the mass of the CP-odd scalar $A^{0}$ and $\lambda$ is a combination of quartic couplings. The next-to-leading-order term in $\kappa_{V}$ has the opposite sign in the $2 \mathrm{HDM}$ in comparison to the GM model, resulting in suppressed Higgs couplings to vector bosons (as expected for a doublet model). Therefore a measurement of an enhanced coupling ratio $\kappa_{V} \geq 1$ at a precision collider (such as the proposed International Linear Collider) would favour a GM model. A suppressed measurement $\kappa_{V} \leq 1$ would favour a $2 \mathrm{HDM}$, unless the GM scalars are quite light.

Furthermore, the couplings decouple at different rates, as summarized in Table 7.6. While the $2 \mathrm{HDM} \kappa_{V}$ decouples faster than $\kappa_{f}$ or $C_{h h h}$, in the GM model they 
all decouple at the same rate. A coupling which decouples slowly may measurably deviate from the SM value even as the BSM scalars become heavy; therefore a pattern of measured deviations in the couplings will favour a model with a similar pattern of decoupling rates. As a result, a measurement of sizeable deviations from the SM in all three observables would favour a Case B GM model, while a 2HDM would be favoured by a deviation in $\kappa_{f}$ and $C_{h h h}$ with an SM-like $\kappa_{V}$.

\subsubsection{The decoupling of loop-level Higgs decays}

We finally consider the decoupling behavior of the loop-induced couplings of $h$ to $\gamma \gamma$ and $Z \gamma$. As discussed in section 3.2.4, BSM effects from $\kappa_{f}, \kappa_{V}$ and new particles propagating in the loop may all affect these processes. Having just discussed the decoupling behaviour of $\kappa_{f, V}$, we are most interested in the decoupling behaviour of the factors $\Delta \kappa_{\gamma}$ and $\Delta \kappa_{Z \gamma}$ - defined in Eqs. (3.67) and (3.78) respectively - which describe the contributions of new scalar particles in the loop.

These factors are affected by contributions from the $H_{3}^{ \pm}, H_{5}^{ \pm}$, and $H_{5}^{ \pm \pm}$scalars to the loop, as defined by Eqs. (3.59) and (3.70) respectively. ${ }^{8}$ As such, their decoupling behaviour depends on the relevant couplings of $h$ to these charged scalars,

$$
\begin{aligned}
& C_{h H_{3}^{+} H_{3}^{+*}} \simeq\left(4 \lambda_{2}-\lambda_{5}\right) v+\frac{\left(M_{1}^{2}-3 M_{1} M_{2}\right) v}{\mu_{3}^{2}} \\
& C_{h H_{5}^{+} H_{5}^{+*}}=C_{h H_{5}^{++} H_{5}^{++*}} \simeq\left(4 \lambda_{2}+\lambda_{5}\right) v+\frac{3 M_{1} M_{2} v}{\mu_{3}^{2}},
\end{aligned}
$$

where we have kept only the leading term in the decoupling limit. In particular, these triple-scalar couplings approach a constant of order $v$ as $\mu_{3} \rightarrow \infty$. As the $h \rightarrow \gamma \gamma, Z \gamma$ loop integrals are inversely proportional to the loop masses, we may use Eqs. (7.99)

\footnotetext{
${ }^{8}$ The loop-decay processes are discussed in more detail in Sec. 7.6.
} 

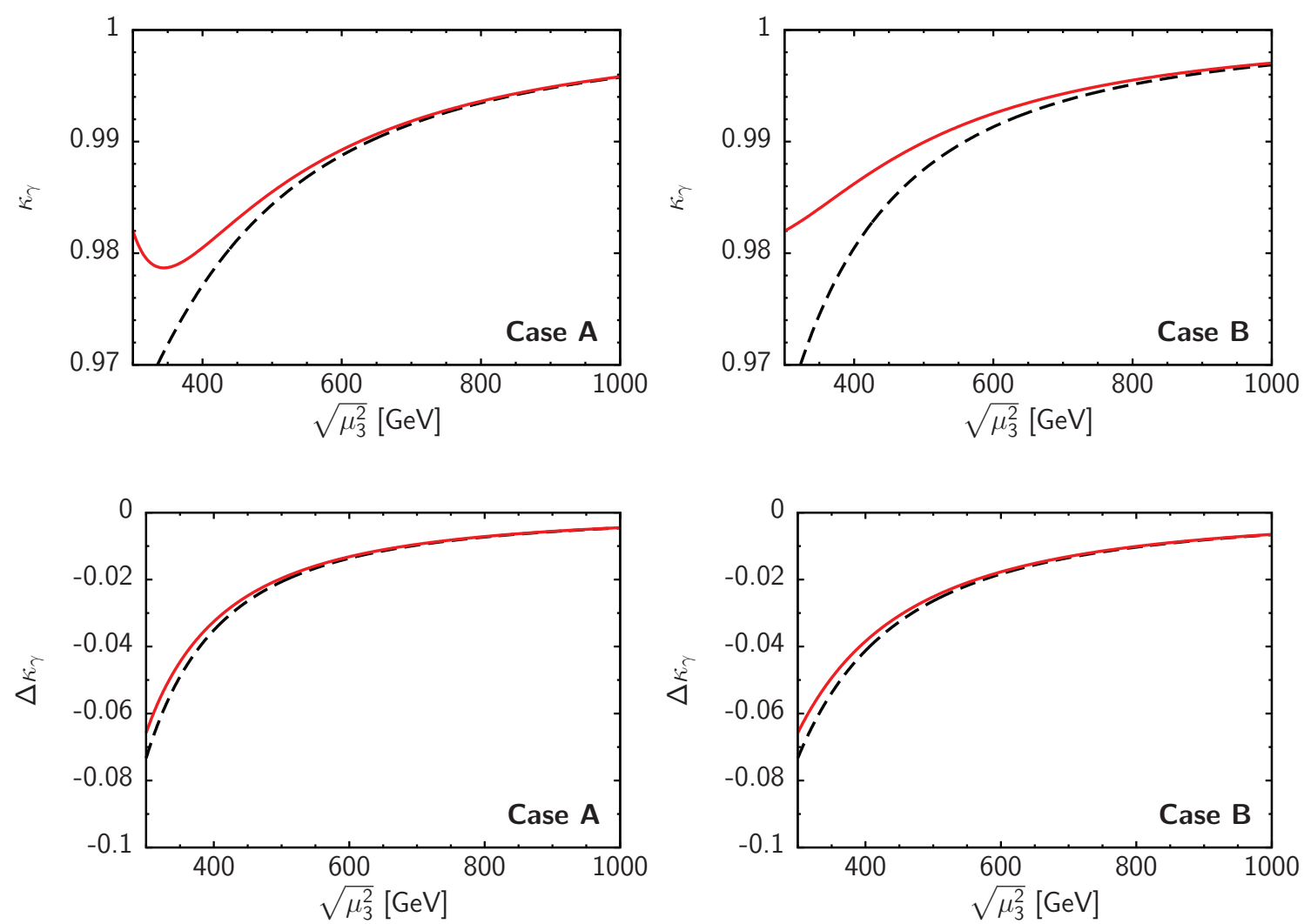

Figure 7.9: Top: The light Higgs coupling modification factor $\kappa_{\gamma}$ as a function of $\mu_{3}$, for cases A (left) and B (right). Bottom: The light Higgs coupling modification factor $\Delta \kappa_{\gamma}$, comprising only the contributions from the non-SM charged scalars in the loop, as a function of $\mu_{3}$. The solid red lines show the exact results, while the dashed black lines are the corresponding expansion formulas.

and (7.100) to show that

$$
\begin{aligned}
\Delta \kappa_{\gamma} & \simeq-\frac{1}{A_{W}^{h \gamma \gamma}+A_{F}^{h \gamma \gamma}} \frac{2 v^{2}}{3 \mu_{3}^{2}}\left[6 \lambda_{2}+\lambda_{5}+\frac{M_{1}^{2}+12 M_{1} M_{2}}{4 \mu_{3}^{2}}\right], \\
\Delta \kappa_{Z \gamma} & \simeq \frac{1}{A_{W}^{Z \gamma}+A_{F}^{Z \gamma}} \frac{1-2 s_{W}^{2}}{2 s_{W} c_{W}} \frac{2 v^{2}}{3 \mu_{3}^{2}}\left[6 \lambda_{2}+\lambda_{5}+\frac{M_{1}^{2}+12 M_{1} M_{2}}{4 \mu_{3}^{2}}\right],
\end{aligned}
$$

Here $A_{W}^{h \gamma \gamma}, A_{F}^{h \gamma \gamma}, A_{W}^{h Z \gamma}$ and $A_{F}^{h Z \gamma}$ are respectively defined by Eqs. (3.60), (3.65), (3.71) and (3.73). Note that in the decoupling limit the charged scalar contributions to the $h \gamma \gamma$ and $h Z \gamma$ loop amplitudes have the same dependence on the GM model parameters, as given in the square brackets in Eqs. (7.109) and (7.110). 

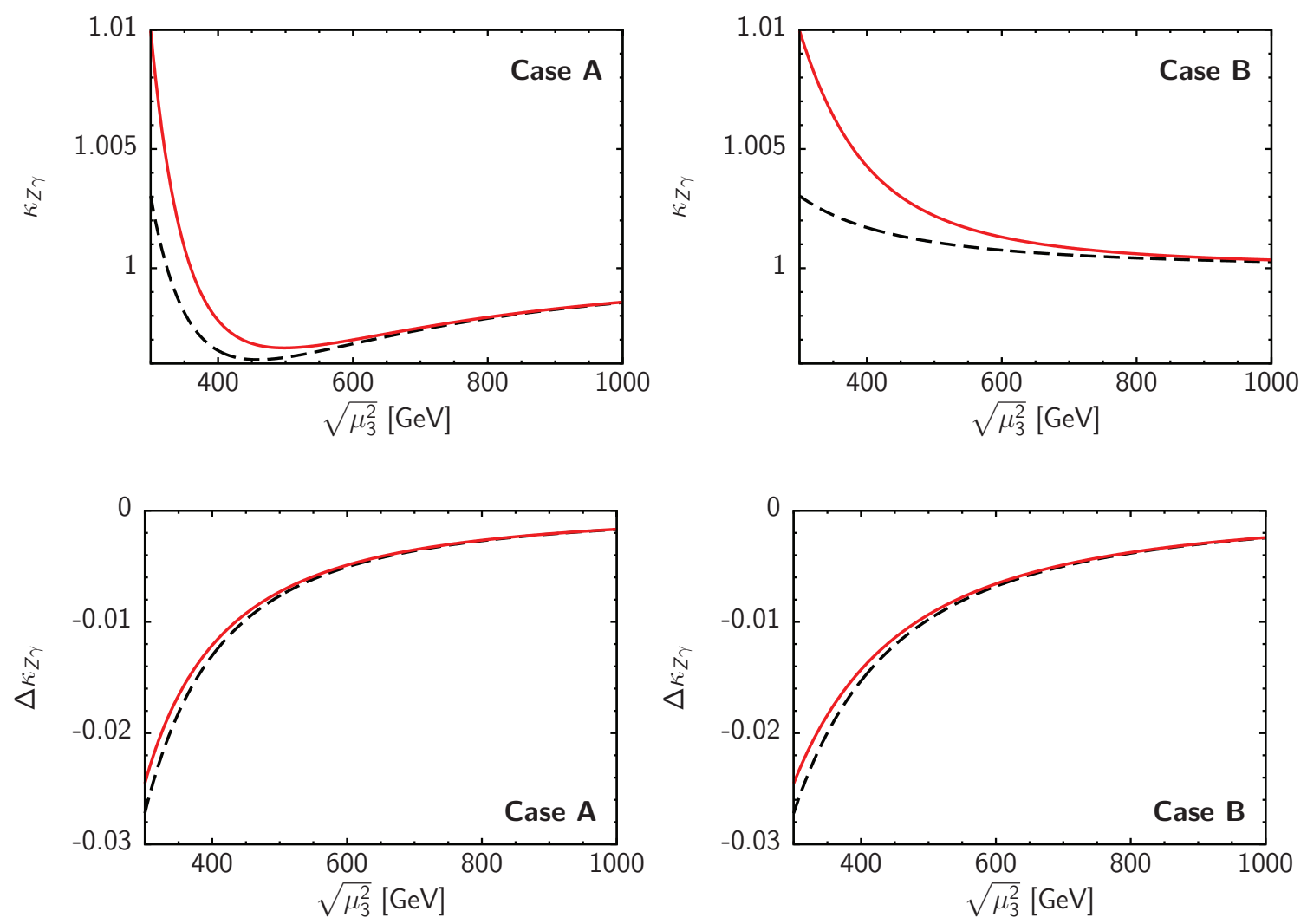

Figure 7.10: Top: The light Higgs coupling modification factor $\kappa_{Z \gamma}$ as a function of $\mu_{3}$, for cases A (left) and B (right). Bottom: The light Higgs coupling modification factor $\Delta \kappa_{Z \gamma}$, comprising only the contributions from the non-SM charged scalars in the loop, as a function of $\mu_{3}$. The solid red lines show the exact results, while the dashed black lines are the corresponding expansion formulas.

In Fig. 7.9 (Fig. 7.10) we illustrate the decoupling behavior of the loop-induced $h \gamma \gamma(h Z \gamma)$ effective coupling $\kappa_{\gamma}\left(\kappa_{Z \gamma}\right)$ defined in Eq. (3.66) (Eq. (3.77)), as well as the scalar loop contributions $\Delta \kappa_{\gamma}\left(\Delta \kappa_{Z \gamma}\right)$. The relatively large deviation at low $\mu_{3}$ between the exact result and the expansion formulas in the upper panels of Figs. 7.9 and 7.10 is due to the similar deviation between the exact $\kappa_{f}$ and its expansion formula (see Fig. 7.7). As shown in the lower panels, the expansion formulas for the scalar loop contributions $\Delta \kappa_{\gamma}$ and $\Delta \kappa_{Z \gamma}$ provide good approximations to the exact results over the range of $\sqrt{\mu_{3}^{2}}$ considered. 


\subsection{Indirect experimental constraints}

In this section we review bounds on the GM parameter space that arise from indirect experimental constraints. Specifically, we consider bounds from oblique parameters, Z-pole observables $\left(R_{b}\right)$, and B-physics processes $\left(b \rightarrow s \gamma, B_{s}^{0}-\bar{B}_{s}^{0}\right.$ meson mixing, and $\left.B_{s}^{0} \rightarrow \mu^{+} \mu^{-}\right)$. Currently the strongest bounds from these indirect constraints arise from $b \rightarrow s \gamma$. We demonstrate the influence of these constraints on the GM parameter space with numerical scans in Sec. 7.4.3.

\subsubsection{Constraints from oblique parameters}

The $S$ parameter [84] is defined by Eq. (3.31). The new contributions to the $S$ parameter in the GM model are given by

$$
\begin{aligned}
\Delta S \equiv & S_{\mathrm{GM}}-S_{\mathrm{SM}} \\
\simeq & \frac{s_{W}^{2} c_{W}^{2} e^{2}}{\pi}\left\{-\frac{1}{12 s_{W}^{2} c_{W}^{2} e^{2}}\left(\log m_{3}^{2}+5 \log m_{5}^{2}\right)+2\left|C_{Z h H_{3}^{0}}\right|^{2} f_{1}\left(m_{h}, m_{3}\right)\right. \\
& +2\left|C_{Z H H_{3}^{0}}\right|^{2} f_{1}\left(m_{H}, m_{3}\right)+2\left(\left|C_{Z H_{5}^{0} H_{3}^{0}}\right|^{2}+2\left|C_{Z H_{5}^{+} H_{3}^{+*}}\right|^{2}\right) f_{1}\left(m_{5}, m_{3}\right) \\
& +\left|C_{Z Z h}\right|^{2}\left[\frac{f_{1}\left(m_{Z}, m_{h}\right)}{2 m_{Z}^{2}}-f_{3}\left(m_{Z}, m_{h}\right)\right]-\left|C_{Z Z h}^{\mathrm{SM}}\right|^{2}\left[\frac{f_{1}\left(m_{Z}, m_{h}^{\mathrm{SM}}\right)}{2 m_{Z}^{2}}-f_{3}\left(m_{Z}, m_{h}^{\mathrm{SM}}\right)\right] \\
& +\left|C_{Z Z H}\right|^{2}\left[\frac{f_{1}\left(m_{Z}, m_{H}\right)}{2 m_{Z}^{2}}-f_{3}\left(m_{Z}, m_{H}\right)\right]+\left|C_{Z Z H_{5}^{0}}\right|^{2}\left[\frac{f_{1}\left(m_{Z}, m_{5}\right)}{2 m_{Z}^{2}}-f_{3}\left(m_{Z}, m_{5}\right)\right] \\
& \left.+2\left|C_{Z W^{+} H_{5}^{+*}}\right|^{2}\left[\frac{f_{1}\left(m_{W}, m_{5}\right)}{2 m_{W}^{2}}-f_{3}\left(m_{W}, m_{5}\right)\right]\right\},
\end{aligned}
$$

where $m_{h}^{\mathrm{SM}}$ is the reference SM Higgs mass for which the oblique parameters are extracted from data, and the loop functions $f_{1}$ and $f_{3}$ are defined in Eqs. (3.39) and (3.48). The GM couplings $C_{i j k}$ are defined in Appendix A.3 without an overall factor of $e^{2}$, and the SM coupling of the Higgs to $Z$ bosons is given by $C_{Z Z h}^{\mathrm{SM}}=v / 2 s_{W}^{2} c_{W}^{2}$, as discussed in Sec. 2.2.3. 
However, it is well known that the $T$ parameter is divergent in the GM model as hypercharge interactions break the $\mathrm{SU}(2)_{R}$ global symmetry at one-loop level [133, 138]. In a more complete theory the one-loop contributions to the $T$ parameter would be compensated by the counterterm of an $\mathrm{SU}(2)_{R}$-breaking quartic coupling in the scalar potential $[40,133]$. In our analysis we take the conservative approach of Ref. [6] and marginalize over the value of $T$ in our $\chi^{2}$ constraint, obtaining a bound from the $S$ parameter alone.

Setting $U=0$, we implement the oblique constraint using the 2-observable $\chi^{2}$ variable defined in Eq. (3.53). Due to the large number of free parameters in this constraint, we use the number of observables as the degrees of freedom, taking $\chi^{2} \leq 4$ at $95 \%$ CL. The observable $S$ is given by Eq. (7.111), and we marginalize over $T$ using Eqs. (3.54-3.55). Our constraint from $S$ agrees numerically with Fig. 1 of Ref. [6].

\subsubsection{Constraints on fermion interactions}

As discussed in Sec. 7.1, the $h, H$, and $H_{3}^{0, \pm}$ GM scalars are all permitted to couple to fermion-anti-fermion pairs due to the mixing between the triplet states $\chi$ and doublet states $\phi$. These scalars may therefore participate in the loop processes contributing to $R_{b}$ and the $B$-meson processes $\left(b \rightarrow s \gamma, B_{s}^{0}-\bar{B}_{s}^{0}\right.$ meson mixing, and $B_{s}^{0} \rightarrow \mu^{+} \mu^{-}$) outlined in Sec. 3.2.5. These observables are dominated by diagrams involving the $H_{3}^{+} \bar{t} b$ scalar coupling to the top quark, as the contributions from neutral scalars are comparatively suppressed by $m_{b} / m_{t}$. As a result, $H_{3}^{+}$is the only BSM scalar in the GM model that contributes significantly to $Z$-pole and $B$-physics observables. These observables each put an upper bound on $v_{\chi}$ (equivalently $\tan \theta_{H}$ ) as a function of $m_{3}$.

The analysis of these constraints is greatly simplified by the observation that 
in the GM model the relevant diagrams are completely analogous to those of the Type-I 2HDM. In the GM model, fermion masses are generated in the same way as in the SM through Yukawa couplings involving the single $\mathrm{SU}(2)_{L}$ doublet; the resulting Feynman rules are tabulated in Appendix A.3. In particular, the GM scalar couplings to fermions have exactly the same structure as those in the Type-I 2HDM [8] with the replacement $\cot \beta \rightarrow \tan \theta_{H}$. Since custodial symmetry requires that the $H_{3}^{+} H_{3}^{-} Z$ coupling be identical to the $\mathrm{H}^{+} \mathrm{H}^{-} Z$ coupling in the $2 \mathrm{HDM}$, the relevant $\mathrm{H}_{3}^{+}$couplings have the same form as those of $H^{+}$in the 2HDM. The $R_{b}$ and $B$-physics constraints on the GM model can therefore be obtained by making the replacements $\cot \beta \rightarrow \tan \theta_{H}$ and $m_{H^{+}} \rightarrow m_{3}$ in the corresponding results for the Type-I 2HDM.

In what follows we place constraints on the GM model by requiring that the GM predictions for $R_{b}, \operatorname{BR}\left(\bar{B} \rightarrow X_{s} \gamma\right), B_{s}^{0}-\bar{B}_{s}^{0}$, and $\overline{\operatorname{BR}}\left(B_{s}^{0} \rightarrow \mu^{+} \mu^{-}\right)$lie within $2 \sigma$ of the experimental measurement. In each case we combine the experimental uncertainty with the GM prediction uncertainty in quadrature. We will refer to these as 'experimental constraints.'

However, in the GM model the $H_{3}^{+}$contributions to $R_{b}, \overline{\mathrm{BR}}\left(B_{s}^{0} \rightarrow \mu^{+} \mu^{-}\right)$, and $\operatorname{BR}\left(\bar{B} \rightarrow X_{s} \gamma\right)$ worsen the agreement with experiment compared to the SM limit (i.e., compared to taking $v_{\chi} \rightarrow 0$ or $m_{3} \rightarrow \infty$ ). As seen in Sec. 3.2.5, the SM limits for these observables are respectively already $0.8 \sigma, 1.0 \sigma$, and 1 to $1.5 \sigma$ away from the experimental measurements. For this reason, we also consider a second, more conservative approach to constraining the parameter space for these three observables: we require that the GM model prediction lie within $2 \sigma$ of the best-fit value obtainable in the GM model, again combining the experimental and GM theoretical uncertainties in quadrature. This best-fit point is the SM-limit for $R_{b}, \overline{\mathrm{BR}}\left(B_{s}^{0} \rightarrow \mu^{+} \mu^{-}\right)$, and $\operatorname{BR}\left(\bar{B} \rightarrow X_{s} \gamma\right)$, and this $2 \sigma$ bound is taken relative to the SM prediction. We will 

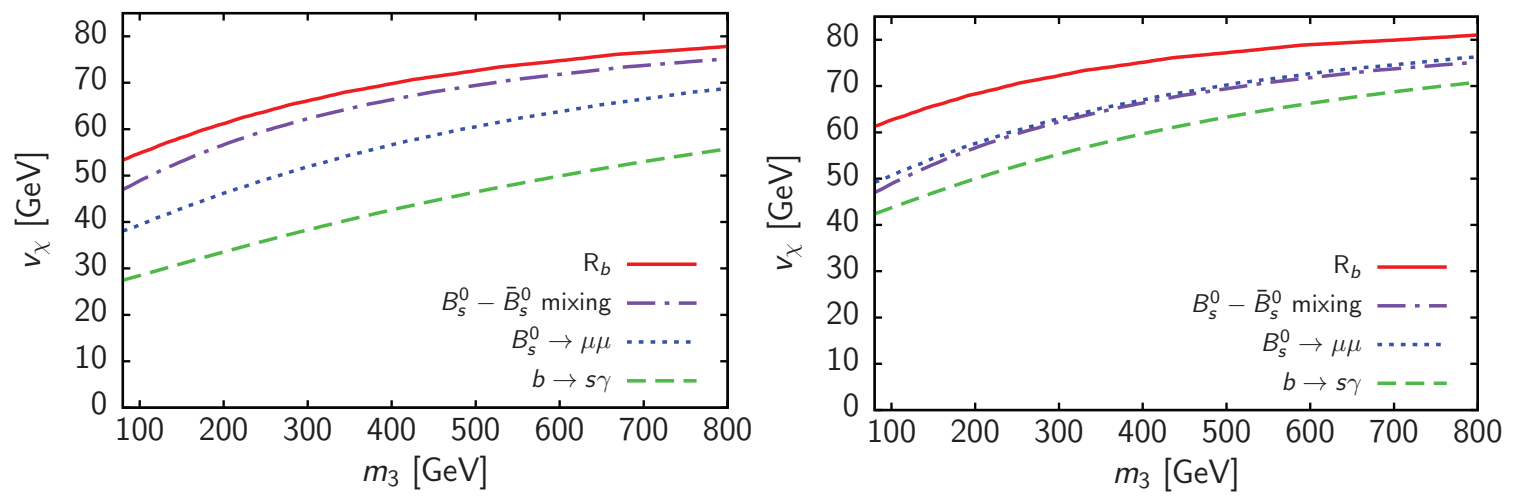

Figure 7.11: Constraints on $m_{3}$ and $v_{\chi}$ in the GM model from $R_{b}, B_{s}^{0}-\bar{B}_{s}^{0}$ mixing, $\overline{\mathrm{BR}}\left(B_{s}^{0} \rightarrow \mu^{+} \mu^{-}\right)$, and $\mathrm{BR}\left(\bar{B} \rightarrow X_{s} \gamma\right)$. The region above each curve is excluded. Left: Experimental constraints. Right: Best-fit constraints.

refer to these more conservative constraints as 'best-fit' constraints. For $B_{s}^{0}-\bar{B}_{s}^{0}$ the best-fit point is the experimental result, and so the best-fit constraint is the same as the experimental constraint.

The left-hand (right-hand) panel of Fig. 7.11 shows the experimental (bestfit) constraints for all observables. ${ }^{9}$ In both cases the tightest constraint arises from $b \rightarrow s \gamma$. The more conservative of the $b \rightarrow s \gamma$ constraints is the best-fit bound taken relative to the $\mathrm{SM}$ prediction, which requires that $v_{\chi} \leq 45 \mathrm{GeV}$ for $m_{3}=100 \mathrm{GeV}$ $v_{\chi} \leq 70 \mathrm{GeV}$ for $m_{3}=800 \mathrm{GeV}$. Details on each process follow.

\section{Radiative bottom quark decay, $b \rightarrow s \gamma$}

The branching ratio $B R\left(\bar{B} \rightarrow X_{s} \gamma\right)$ is defined in Eq. (3.100). The charged Higgs contributions for this branching ratio in the Type-I 2HDM were first calculated by

\footnotetext{
${ }^{9}$ With the exception of top quark pole mass $m_{t}$, we choose the input parameters for all our numerical results from the 2014 Review of Particle Physics [43]. For $m_{t}$, we use the first combination of Tevatron and LHC measurements of the top quark mass [150]. In particular, we set $G_{F}=$ $1.1663787 \times 10^{-5} \mathrm{GeV}^{-2}, \alpha_{\mathrm{em}}=1 / 127.94, \alpha_{s}=0.1184, \bar{m}_{c}\left(m_{c}\right)=1.275 \mathrm{GeV}, \bar{m}_{b}\left(m_{b}\right)=4.18 \mathrm{GeV}$, $m_{Z}=91.1876 \mathrm{GeV}$ and $m_{t}=172.9 \mathrm{GeV}$. In addition, we obtain the dependent parameters $m_{W}=$ $79.83 \mathrm{GeV}$ and $s_{W}^{2}=0.2336$ at tree level. The input files of SuperIso v3.3 (which by default uses inputs from the 2011 Review of Particle Physics [151]) have been edited accordingly.
} 
Ref. [152], and are now known up to NLO in QCD [153]. The relevant contributions from BSM states are those that affect the effective Wilson coefficient, $C_{7, \mathrm{BSM}}^{(0) e f f}\left(\mu_{b}\right)$ (see Eq. (3.101)). As indicated in Sec. 3.2.5, this effective coefficient defined at scale $\mu_{b} \sim m_{b}$ may be related to other Wilson coefficients at the renormalization scale $\mu_{W} \sim m_{W}$. As a result, the additional contributions of the BSM states to all Wilson coefficients $C_{i}\left(\mu_{W}\right)$ may be relevant, depending on the order of the calculation.

To leading order, only contributions to $C_{7}^{(0)}\left(\mu_{W}\right)$ and $C_{8}^{(0)}\left(\mu_{W}\right)$ are relevant. In the GM model, the additional contributions to these coefficients are as follows:

$$
\delta C_{7,8}^{(0, \mathrm{GM})}\left(\mu_{W}\right)=\tan ^{2} \theta_{H}\left(\frac{F_{7,8}^{(1)}\left(x_{t 3}\right)}{3}-F_{7,8}^{(2)}\left(x_{t 3}\right)\right),
$$

where $x_{t W}=\bar{m}_{t}\left(\mu_{W}\right)^{2} / m_{W}^{2}, x_{t 3}=\bar{m}_{t}\left(\mu_{W}\right)^{2} / m_{3}^{2}$, and $\bar{m}_{t}\left(\mu_{W}\right)$ is the $\overline{\mathrm{MS}}$ top quark running mass renormalized at scale $\mu_{W}$. The functions $F_{7,8}^{(1)}$ are defined in Eqs. (3.1033.104), and the functions $F_{7,8}^{(2)}$ are defined as

$$
\begin{aligned}
& F_{7}^{(2)}(x)=\frac{x(3-5 x)}{12(x-1)^{2}}+\frac{x(3 x-2)}{6(x-1)^{3}} \ln x, \\
& F_{8}^{(2)}(x)=\frac{x(3-x)}{4(x-1)^{2}}-\frac{x}{2(x-1)^{3}} \ln x .
\end{aligned}
$$

Equations (7.112)-(7.114) may be used with Eqs. (3.101)-(3.104) to obtain a LO prediction for $B R\left(\bar{B} \rightarrow X_{s} \gamma\right)$ in the GM model.

However, because this observable will provide the most stringent constraint on the GM model parameter space, we will make use of the full implementation of the SM and 2HDM contributions in the public code SuperIso v3.3 [154-156], which is based on the calculations in Refs. [112,157]. SuperIso calls the code 2HDMC v1.6.4 [158] for spectrum calculations within the Type-I 2HDM.

The error on this prediction can be estimated by scaling the error on the SM prediction by the ratio $R_{b \rightarrow s \gamma}^{\mathrm{GM}}$ of the BSM prediction to the SM prediction (as defined 
in Eq. (3.101)). For consistency, we will also use the equivalent SM $b \rightarrow s \gamma$ prediction from SuperIso, rather than the predictions of Eq. (3.106). In the SM limit of $v_{\chi} \rightarrow 0$ (or $\left.m_{3} \rightarrow \infty\right)$ the calculation of $\mathrm{BR}\left(\bar{B} \rightarrow X_{s} \gamma\right)$ by SuperIso yields a prediction of

$$
\mathrm{BR}\left(\bar{B} \rightarrow X_{s} \gamma\right)_{\text {SM limit }}=3.11 \times 10^{-4},
$$

where we have used the input parameters given in footnote 9 . The difference compared to the SM predictions quoted in Eq. (3.106) is primarily due to differences in the input parameters - particularly $m_{b}$ and $m_{c}[159]$ — and is within the $\pm 3 \%$ parametric uncertainty [112]. We take the total theoretical uncertainty on this SM prediction to be $\pm 0.23 \times 10^{-4}$ from Ref. [112]. Combining this in quadrature with the experimental uncertainty yields a total uncertainty of $\pm 0.34 \times 10^{-4}$.

The charged Higgs contribution to $\mathrm{BR}\left(\bar{B} \rightarrow X_{s} \gamma\right)$ in the GM model interferes destructively with the SM contribution, leading to a smaller predicted value for $\mathrm{BR}\left(\bar{B} \rightarrow X_{s} \gamma\right)$ than in the SM. Because the SM prediction is already below the experimental central value, the best agreement with the experimental measurement in the GM model occurs in the limit $v_{\chi} \rightarrow 0$ or $m_{3} \rightarrow \infty$ (i.e., the SM limit). As the SM limit of Eq. (7.115) yields a prediction that is $1.3 \sigma$ from the experimental measurement given in Eq. (3.105), the resulting experimental bound on the GM $\left(m_{3}, v_{\chi}\right)$ plane is quite strong, as can be seen in the left panel of Fig. 7.11. This $2 \sigma$ experimental constraint corresponds to $\operatorname{BR}\left(\bar{B} \rightarrow X_{s} \gamma\right)>2.88 \times 10^{-4}$. In comparison, the $2 \sigma$ best-fit constraint with respect to the the SM limit (Eq. (7.115)) yields $\operatorname{BR}\left(\bar{B} \rightarrow X_{s} \gamma\right)>2.48 \times 10^{-4}$. This is shown in the right panel of Fig. 7.11 together with the best-fit constraints from the other observables. In either case, $\operatorname{BR}\left(\bar{B} \rightarrow X_{s} \gamma\right)$ is the strongest constraint on these parameters.

Because of the large theoretical uncertainty on $\operatorname{BR}\left(\bar{B} \rightarrow X_{s} \gamma\right)$ and the sensitivity of the resulting constraint to the particular choice of input parameters and 
the handling of partial higher-order corrections, we consider it safer to take the more conservative approach and apply the best-fit constraint from $\operatorname{BR}\left(\bar{B} \rightarrow X_{s} \gamma\right)$ as our primary constraint on the $\left(m_{3}, v_{\chi}\right)$ plane. We will nevertheless also show the effect of applying the experimental $b \rightarrow s \gamma$ constraint in our numerical scans.

\section{$B$ meson mixing}

The oscillation frequency of a $B_{q}^{0}$ meson due to meson-antimeson mixing is defined in Eq. (3.84). As indicated by Eq. (3.86), the effect of charged scalars on $B_{q}^{0}-\bar{B}_{q}^{0}$ mixing predominantly affects the kinematic function $I$. The particular case of $B_{d}^{0}$ mixing in a Type-I 2HDM has been studied in Ref. [160] by adapting results of similar processes on $K^{0}-\bar{K}^{0}$ mixing [161]. These results can be extended to the $B_{s}^{0}-\bar{B}_{s}^{0}$ system, which is more constraining than the $B_{d}^{0}-\bar{B}_{d}^{0}$ system due to smaller uncertainties in both the experimental measurement and the SM prediction [162].

To leading order, in the GM model the function $I$ becomes

$$
I_{\mathrm{GM}}=I_{W W}\left(x_{t W}\right)+\tan ^{2} \theta_{H} I_{W H}\left(x_{t W}, x_{t 3}, x_{3 W}\right)+\tan ^{4} \theta_{H} I_{H H}\left(x_{t 3}\right) .
$$

Here $x_{3 W}=m_{3}^{2} / m_{W}^{2}, x_{t W}=\bar{m}_{t}^{2}\left(\mu_{t}\right) / m_{W}^{2}$, and $x_{t 3}=\bar{m}_{t}^{2}\left(\mu_{t}\right) / m_{3}^{2}$. We set the top mass renormalization scale $\mu_{t}=m_{t}$, where $m_{t}$ is the top quark pole mass. The Inami-Lim functions $I_{H H}$ and $I_{W H}$ are given by [98]

$$
\begin{aligned}
I_{H H}(x) & =x\left[\frac{1+x}{(1-x)^{2}}+\frac{2 x \log x}{(1-x)^{3}}\right] \\
I_{W H}(x, y, z) & =y\left[\frac{(2 z-8) \log y}{(1-y)^{2}(1-z)}+\frac{6 z \log x}{(1-x)^{2}(1-z)}-\frac{8-2 x}{(1-y)(1-x)}\right] .
\end{aligned}
$$

The function $I_{W W}$ is the SM contribution to $I$, and is defined in Eq. (3.85).

Under the assumption that the overall coefficients do not vary substantially due to new scalar contributions, a prediction for $\Delta m_{B_{s}}^{\mathrm{GM}}$ in the GM model may be 
obtained using the ratio defined in Eq. (3.86),

$$
R_{\Delta m}^{\mathrm{GM}} \equiv \frac{\Delta m_{B_{s}}^{\mathrm{GM}}}{\Delta m_{B_{s}}^{\mathrm{SM}}}=1+\frac{\tan ^{2} \theta_{H} I_{W H}\left(x_{t W}, x_{t 3}, x_{3 W}\right)+\tan ^{4} \theta_{H} I_{H H}\left(x_{t 3}\right)}{I_{W W}\left(x_{t W}\right)} .
$$

Since $I_{W W}, I_{W H}$ and $I_{H H}$ are all positive, the GM model contribution interferes constructively with the SM contribution. Because the theoretical uncertainty on the mass splitting is due almost entirely to uncertainties in the coefficients of $I$ in Eq. (3.84), we scale the SM theoretical uncertainty $\delta \Delta m_{B_{s}}^{\mathrm{SM}}$ by $R_{\Delta m}^{\mathrm{GM}}$ to obtain the theoretical uncertainty in the GM model, i.e., $\delta \Delta m^{\mathrm{GM}}=R_{\Delta m}^{\mathrm{GM}} \cdot \delta \Delta m^{\mathrm{SM}}$.

In the case of $B_{s}^{0}-\bar{B}_{s}^{0}$ mixing, the charged Higgs contributions in the GM model increase the predicted value of $R_{\Delta m}$, so that the best-fit value and the experimental central value are the same. Therefore the best-fit and experimental bounds are both given by Eq. (3.90), $R_{\Delta m}^{\mathrm{GM}} \leq 1.46$. The resulting constraint on the $\left(m_{3}, v_{\chi}\right)$ plane is shown in the left and right panels of Fig. 7.11. It is slightly more constraining than the bounds from $R_{b}$, and is about the same as the experimental bound from $B_{s}^{0} \rightarrow \mu^{+} \mu^{-}$. In both cases the bound is weaker than that from $b \rightarrow s \gamma$.

In these calculations we have used the SM prediction given by Eq. (3.88), which has an error of $\pm 2.6 \mathrm{ps}^{-1}$ as discussed in Sec. (3.2.5). If we were to use the less-conservative error of $\pm 1.5 \mathrm{ps}^{-1}$ obtained in Ref. [102], the uncertainty on $R_{\Delta m}^{\exp }$ in Eq. (3.89) becomes 0.089 and the bound would tighten to match the experimental bound from $B_{s}^{0} \rightarrow \mu^{+} \mu^{-}$in the left-hand panel. If we were instead to use the central value $\Delta m_{B_{s}}^{\mathrm{SM}}=21.7 \pm 2.6 \mathrm{ps}^{-1}$, the best-fit reference point would become the SM prediction, $R_{\Delta m}^{\exp }=0.817 \pm 0.098$, and the experimental and best-fit bounds would each be slightly stronger than the corresponding bounds from $b \rightarrow s \gamma$. This variability illustrates the very large remaining theoretical uncertainty in this observable. An improvement in the constraint from $B_{s}^{0}-\bar{B}_{s}^{0}$ mixing relies on an improved lattice QCD determination of the parameters $f_{B_{s}} B_{B_{s}}^{1 / 2}$. 


\section{$B$ meson decays to $\mu^{+} \mu^{-}$}

The branching ratio of $B_{s}^{0} \rightarrow \mu^{+} \mu^{-}$is defined in Eq. (3.91). The contribution from the new GM states can be defined as a ratio to the SM as in Eq. (3.94); it consists of a ratio $\bar{R}_{B_{s}^{0} \rightarrow \mu^{+} \mu^{-}}^{\mathrm{GM}}=\left|C_{10}^{\mathrm{GM}} / C_{10}^{\mathrm{SM}}\right|^{2}$ of the Wilson coefficients $C_{10}$ in the GM and $\mathrm{SM}$, where $C_{10}^{\mathrm{SM}}$ is defined in Eq. (3.95). A full leading-order computation of the average time-integrated branching ratio $\overline{\mathrm{BR}}\left(B_{s}^{0} \rightarrow \mu^{+} \mu^{-}\right)$in the Aligned 2HDM [163] was recently performed in Ref. [105]. The calculation can be easily specialized to the Type-I $2 \mathrm{HDM}$ and hence to the GM model; the Wilson coefficient $C_{10}$ in the GM model is then given by

$$
C_{10}^{\mathrm{GM}}=C_{10}^{\mathrm{SM}}+\tan ^{2} \theta_{H} \frac{x_{t W}}{8}\left[\frac{x_{t 3}}{1-x_{t 3}}+\frac{x_{t 3} \log x_{t 3}}{\left(1-x_{t 3}\right)^{2}}\right]
$$

with $x_{t W}=\bar{m}_{t}^{2}\left(\mu_{t}\right) / m_{W}^{2}$ and $x_{t 3} \equiv m_{t}^{2}\left(\mu_{t}\right) / m_{3}^{2}$ as before. Here $\mu_{t}=m_{t}$ is set to the top quark pole mass. The theoretical uncertainty on the resulting GM branching ratio is taken to be $\delta \overline{\mathrm{BR}}\left(B_{s}^{0} \rightarrow \mu^{+} \mu^{-}\right)_{\mathrm{GM}}=\bar{R}_{B_{s}^{0} \rightarrow \mu^{+} \mu^{-}}^{\mathrm{GM}} \cdot \delta \overline{\mathrm{BR}}\left(B_{s}^{0} \rightarrow \mu^{+} \mu^{-}\right)_{\mathrm{SM}}$.

The $2 \sigma$ experimental constraint of $\bar{R}_{B_{s}^{0} \rightarrow \mu^{+} \mu^{-}}^{\mathrm{GM}} \leq 1.21$ (given by Eq. (3.99)) is shown in the left-hand panel of Fig. 7.11; it is stronger than the corresponding constraints from $R_{b}$ and $B_{s}^{0}-\bar{B}_{s}^{0}$ mixing, but remains weaker than the experimental $b \rightarrow s \gamma$ constraint. However, as discussed in Sec. 3.2.5, this observable has the best prospects for improvement in the near future. Results from the LHCb experiment are expected to reduce the uncertainty from $25 \%$ to less than $10 \%$ after 10 years of running. In comparison, the $b \rightarrow s \gamma$ uncertainty is only expected to improve to $7 \%$ from $7.2 \%[113]$.

The GM model contribution to $C_{10}$ in Eq. (7.120) is always negative, leading to constructive interference with the SM contribution and increasing the prediction for $\bar{R}_{B_{s}^{0} \rightarrow \mu^{+} \mu^{-}}$compared to its value in the SM. As the SM value is already $1.0 \sigma$ 
larger than the experimental value $\left(\bar{R}_{B_{s}^{0} \rightarrow \mu^{+} \mu^{-}}^{\exp }<1\right)$, the best agreement with the experimental measurement of $\overline{\mathrm{BR}}\left(B_{s}^{0} \rightarrow \mu^{+} \mu^{-}\right)$in the GM model occurs in the limit $v_{\chi} \rightarrow 0$ or $m_{3} \rightarrow \infty$ (i.e., the SM limit). The best-fit $2 \sigma$ bound taken relative to the SM prediction yields a best-fit constraint of $\bar{R}_{B_{s}^{0} \rightarrow \mu^{+} \mu^{-}} \leq 1.43$, which is shown in the right-hand panel of Fig. 7.11. The best-fit constraint from $\overline{\mathrm{BR}}\left(B_{s}^{0} \rightarrow \mu^{+} \mu^{-}\right)$is stronger than that from $R_{b}$, comparable to $\bar{B}_{s}^{0}-B_{s}^{0}$ mixing, and weaker than that from $\operatorname{BR}(b \rightarrow s \gamma)$.

\section{$Z$-decay to $b \bar{b}\left(\mathbf{R}_{b}\right)$}

The correction $\delta R_{b}$ to the Z-pole observable $R_{b}$ defined in Eq. (3.79) has been calculated in the 2HDM in Refs. $[164,165]$. The results for the Type-I 2HDM have previously been adapted to the GM model in Refs. [94,144]. Following Ref. [94], the one-loop charged Higgs correction to $R_{b}^{\mathrm{SM}}$ (the $\delta R_{b}^{\mathrm{GM}}$ of Eq. (3.80)) can be written as

$$
\begin{aligned}
\delta R_{b}^{\mathrm{GM}} & \approx-0.7785 \delta g_{\mathrm{GM}}^{L} \\
& \simeq \frac{0.7785}{64 \pi^{2}}\left(\frac{e^{3}}{s_{W}^{3} c_{W}}\right) \tan ^{2} \theta_{H} x_{t W}\left(\frac{x_{t 3}}{1-x_{t 3}}+\frac{x_{t 3} \log x_{t 3}}{\left(1-x_{t 3}\right)^{2}}\right),
\end{aligned}
$$

where $x_{t W}=\bar{m}_{t}^{2}\left(\mu_{t}\right) / m_{W}^{2}, x_{t 3}=\bar{m}_{t}^{2}\left(\mu_{t}\right) / m_{3}^{2}$, and we neglect $m_{Z}$ in the loop calculation. ${ }^{10}$ Here $\bar{m}_{t}$ is the $\overline{\mathrm{MS}}$ running mass evaluated at $\mu_{t}=m_{Z}$. We neglect $\delta g^{R}$ in Eq. (7.121) because $\delta g_{\mathrm{GM}}^{R}$ is suppressed by a factor of $m_{b}^{2} / m_{t}^{2}$ compared to $\delta g_{\mathrm{GM}}^{L}[94,144]$.

One may note that the structure of Eq. (7.121) is very similar to that for the $H_{3}^{+}$contribution to the $C_{10}^{\mathrm{GM}}$ Wilson coefficient in the $\overline{\mathrm{BR}}\left(B_{s}^{0} \rightarrow \mu^{+} \mu^{-}\right)$prediction (see Eq. (7.120)); they have the same dependence on $m_{3}$ and $\tan \theta_{H}$. This is because the charged Higgs contribution to $C_{10}^{\mathrm{GM}}$ comes from the same $Z$ penguin diagrams

\footnotetext{
${ }^{10}$ Full expressions including the $m_{Z}$ dependence have been given in Refs. $[94,144]$.
} 
as in $R_{b}$, but with a generation-changing $H^{+} \bar{t}_{R} s_{L}$ vertex in place of the generationconserving $H^{+} \bar{t}_{R} b_{L}$ vertex (and $p_{Z}^{2}=M_{B_{s}}^{2} \simeq 0$ ).

The experimental measurement and SM prediction are listed in Eqs. (3.81) and (3.82). The 2-sigma experimental bound is $R_{b}^{\mathrm{GM}}=R_{b}^{\mathrm{SM}}+\delta R_{b}^{\mathrm{GM}}>0.21495$ as given by Eq. (3.83). In this case we combine the experimental and SM prediction uncertainties in quadrature, as we estimate the uncertainty on the GM prediction to be the same as that of the SM. However, one will note that the SM prediction is $0.8 \sigma$ below the measured value; as the GM correction interferes destructively with the SM contribution, it increases the discrepancy between theory and experiment. The best agreement with the experimental measurement of $R_{b}$ in the GM model occurs in the SM limit $\left(v_{\chi} \rightarrow 0, m_{3} \rightarrow \infty\right)$. Requiring that the GM prediction for $R_{b}$ lie within $2 \sigma$ of the SM value yields a best-fit constraint of $R_{b}^{\mathrm{GM}}>0.21443$.

As we see in Fig. 7.11, $R_{b}$ provides the weakest non-oblique experimental and best-fit constraints. Furthermore, substantial improvement in the $R_{b}$ experimental measurement is unlikely in the near future, as this would require better $Z$-pole measurements using a next-generation $e^{+} e^{-}$collider such as the International Linear Collider (ILC) with the GigaZ option [96].

\subsubsection{Numerical results from theoretical and indirect exper- imental constraints}

The theoretical, $S$-parameter, $b \rightarrow s \gamma$, and $B_{s}^{0} \rightarrow \mu^{+} \mu^{-11}$ constraints considered in Secs. 7.2 and 7.4 have been implemented in a public code called GMCalc [166].

\footnotetext{
${ }^{11}$ The $B_{s}^{0} \rightarrow \mu^{+} \mu^{-}$constraint has been implemented in GMCalc in anticipation of new results from LHCb, which may make this bound stronger than the $b \rightarrow s \gamma$ constraint.
} 

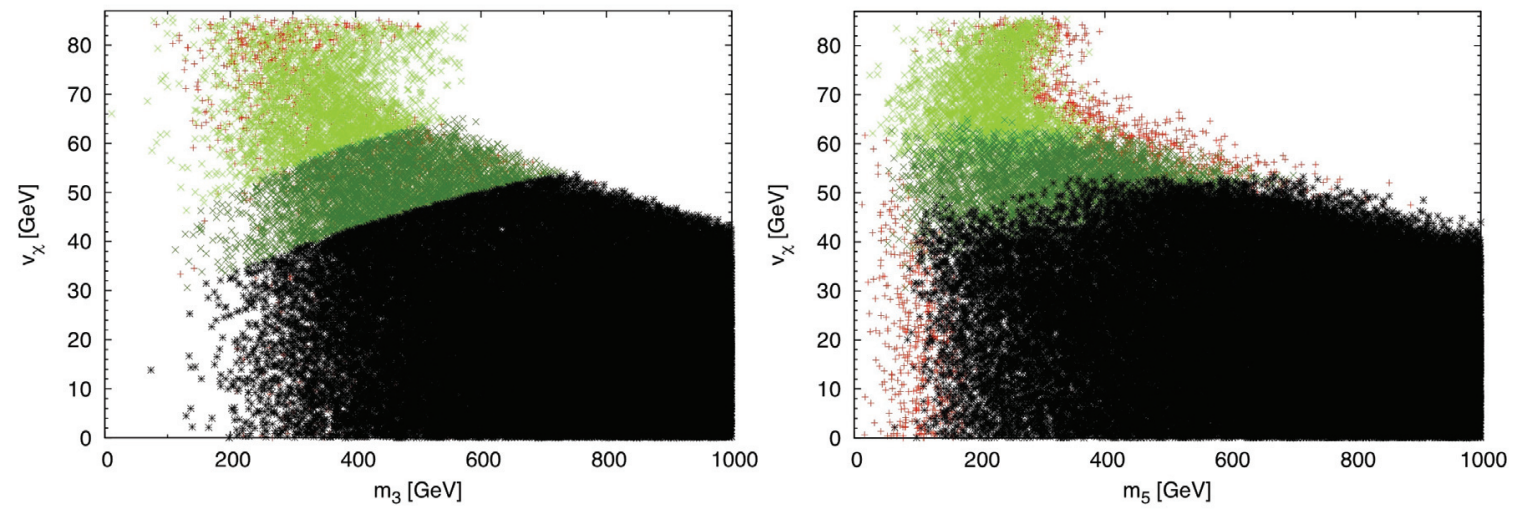

Figure 7.12: Effect of the experimental constraints on $\mathrm{BR}(b \rightarrow s \gamma)$ and the $S$ parameter on $v_{\chi}$, as a function of $m_{3}$ (left) and $m_{5}$ (right). The black points are allowed. The red (medium gray) +-shaped points are eliminated by the $S$ parameter constraint. The light green (light gray) $\times$-shaped points are eliminated by the best-fit $b \rightarrow s \gamma$ constraint. The dark green (dark gray) $\times$-shaped points would be eliminated by the experimental $b \rightarrow s \gamma$ constraint.

Using this code, we illustrate the effects of the theoretical, $S$-parameter, and $b \rightarrow$ $s \gamma$ constraints on the parameter space of the GM model in Figs. 7.12-7.16. Each constraint is applied according to the methodology discussed in the relevant section. We require that either $h$ or $H$ has mass $125 \mathrm{GeV}$ and set the SM Higgs vev $v$ using $G_{F}$. We take $\mu_{3}^{2} \leq(1200 \mathrm{GeV})^{2}$, which fully populates the mass ranges shown in the figures below. Additional points generated by a dedicated scan with $\mu_{3}^{2} \leq(200 \mathrm{GeV})^{2}$ are included in Fig. 7.16 in order to better populate the low-mass region.

We start by showing the effect of the $b \rightarrow s \gamma$ measurement on the $\left(m_{3}, v_{\chi}\right)$ plane in the left panel of Fig. 7.12. We see that, due to its interplay with the decoupling effect of falling $v_{\chi}$ with increasing triplet masses, the experimental $b \rightarrow s \gamma$ constraint eliminates all model points with $v_{\chi} \gtrsim 54 \mathrm{GeV}$ and the best-fit $b \rightarrow s \gamma$ constraint eliminates all model points with $v_{\chi} \gtrsim 65 \mathrm{GeV}$. We will take this latter, more conservative constraint for our conclusions.

This is reflected in the right panel of Fig. 7.12, where we plot $v_{\chi}$ as a function 

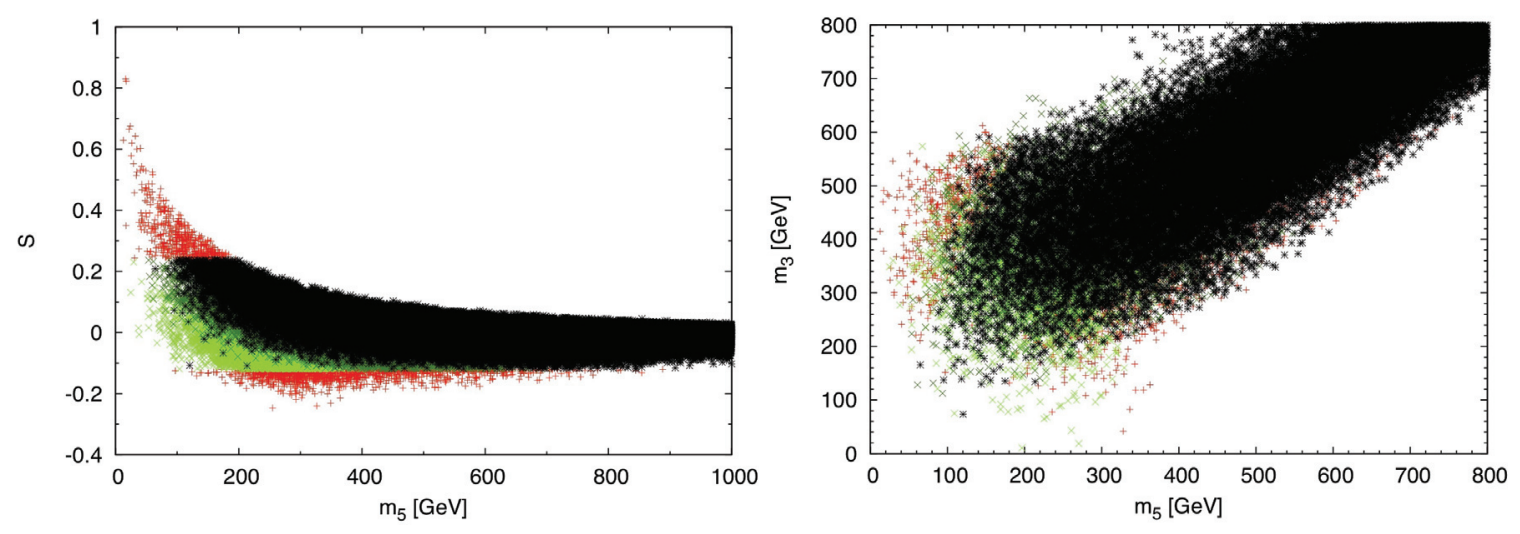

Figure 7.13: Effect of the experimental constraints on $\mathrm{BR}(b \rightarrow s \gamma)$ and the $S$ parameter as a function of $m_{5}$. The colour codes are the same as in Fig. 7.12.

of $m_{5}$. Because $m_{5} \neq m_{3}$ in general, values of $v_{\chi}$ up to the limit of $\sim 65 \mathrm{GeV}$ are allowed even for $m_{5}$ masses as low as $100 \mathrm{GeV}$ under the best-fit $b \rightarrow s \gamma$ constraint. In the right panel of Fig. 7.12 we also see the effect of the $S$ parameter constraint, which eliminates a few model points at very low $m_{5}$, as well as moderate to high $m_{5}$ and high $v_{\chi}$. This is illustrated in more detail in Fig. 7.13. In particular, points with very low values of $m_{5}$ tend to have a large splitting between $m_{5}$ and $m_{3}$, which leads to large positive values of the $S$ parameter.

In Fig. 7.14 we show the effect of the constraints from $\operatorname{BR}(b \rightarrow s \gamma)$ and the $S$ parameter on the allowed ranges of the couplings of the $125 \mathrm{GeV}$ Higgs boson $h$ to $W$ and $Z$ boson pairs and to fermion pairs. ${ }^{12}$ In particular, for maximal $v_{\chi}$ and $|\sin \alpha| \sim 1$ (corresponding to $h$ being entirely composed of triplet states), $\kappa_{V}$ can be as large as 1.6. This maximal value is reduced to $\kappa_{V} \lesssim 1.36$ by the upper bound on $v_{\chi}$

\footnotetext{
${ }^{12}$ For a small number of points in our scan, the $125 \mathrm{GeV}$ Higgs boson is $H$, and the lighter custodial singlet $h$ has a mass below $125 \mathrm{GeV}$. In these cases, we plot the coupling scaling factors $\kappa_{V}$ and $\kappa_{f}$ that represent the $H V V(V=W, Z)$ and $H \bar{f} f$ couplings, respectively, normalized to their SM values. These couplings are given in this case by

$$
\kappa_{V}=\sin \alpha \frac{v_{\phi}}{v}+\frac{8}{\sqrt{3}} \cos \alpha \frac{v_{\chi}}{v}, \quad \kappa_{f}=\sin \alpha \frac{v}{v_{\phi}} .
$$



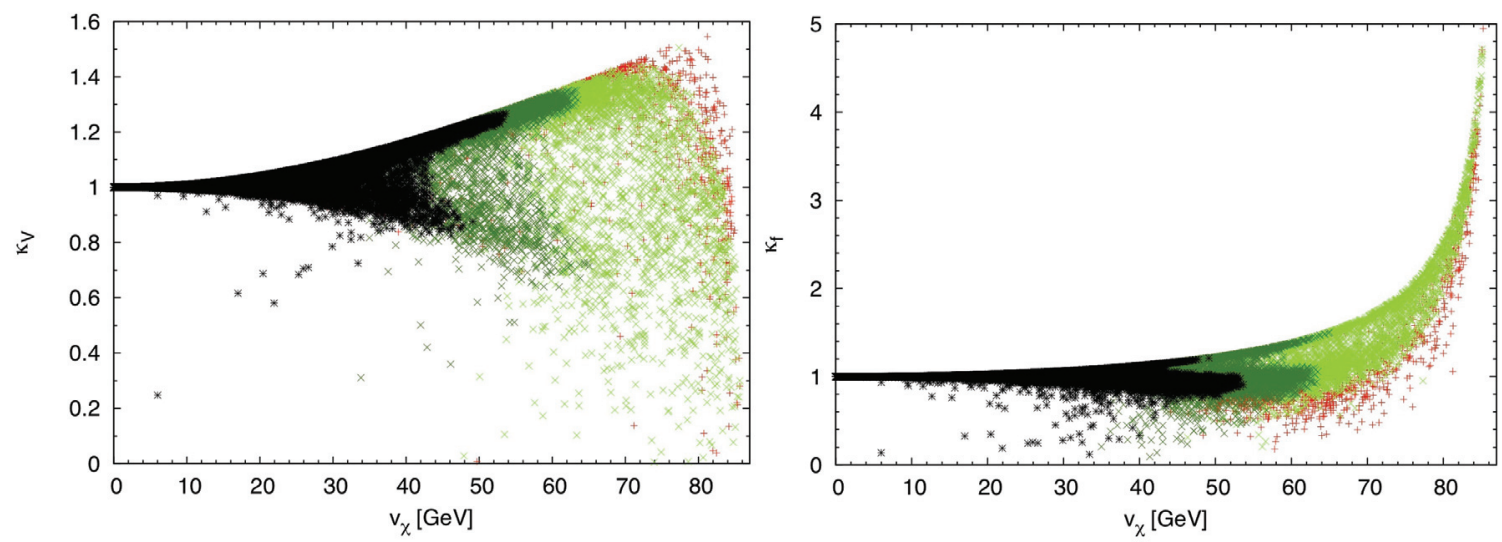

Figure 7.14: Effect of the experimental constraints on $\mathrm{BR}(b \rightarrow s \gamma)$ and the $S$ parameter on the couplings of the $125 \mathrm{GeV}$ Higgs boson $h$ to $W$ and $Z$ boson pairs (left) and fermion pairs (right), shown as a function of $v_{\chi} \cdot \kappa_{V}\left(\kappa_{f}\right)$ is defined as the coupling of $h$ to $V V(\bar{f} f)$ normalized to the corresponding SM Higgs boson coupling. The colour codes are the same as in Fig. 7.12.

imposed by the best-fit $b \rightarrow s \gamma$ constraint, as shown in the left panel of Fig. 7.14. It would be reduced even further to $\kappa_{V} \lesssim 1.28$ by the experimental $b \rightarrow s \gamma$ constraint.

Similarly, $\kappa_{f}$ can be significantly enhanced in the GM model if $v_{\phi}$ is small. By limiting the maximum size of $v_{\chi}$, the best-fit $b \rightarrow s \gamma$ constraint puts a lower bound on $v_{\phi}$, and thereby imposes an upper bound on $\kappa_{f}$ of $\kappa_{f} \lesssim 1.49$, as shown in the right panel of Fig. 7.14. The experimental constraint on $b \rightarrow s \gamma$ would reduce this further to $\kappa_{f} \lesssim 1.20$. The $S$ parameter measurement does not further constrain the allowed ranges of either $\kappa_{V}$ or $\kappa_{f}$ in a significant way once either of the $b \rightarrow s \gamma$ constraints have been applied.

Finally we show the allowed range of correlations between $\kappa_{V}$ and $\kappa_{f}$ in Fig. 7.15. We note in particular that the GM model can accommodate simultaneous enhancements of both $\kappa_{V}$ and $\kappa_{f}$. Such enhancements are constrained by the best-fit $b \rightarrow s \gamma$ bound to lie below $\kappa_{V} \simeq \kappa_{f} \simeq 1$.18. The experimental $b \rightarrow s \gamma$ constraint would reduce this to about 1.09. As discussed at the beginning of the chapter, such a 


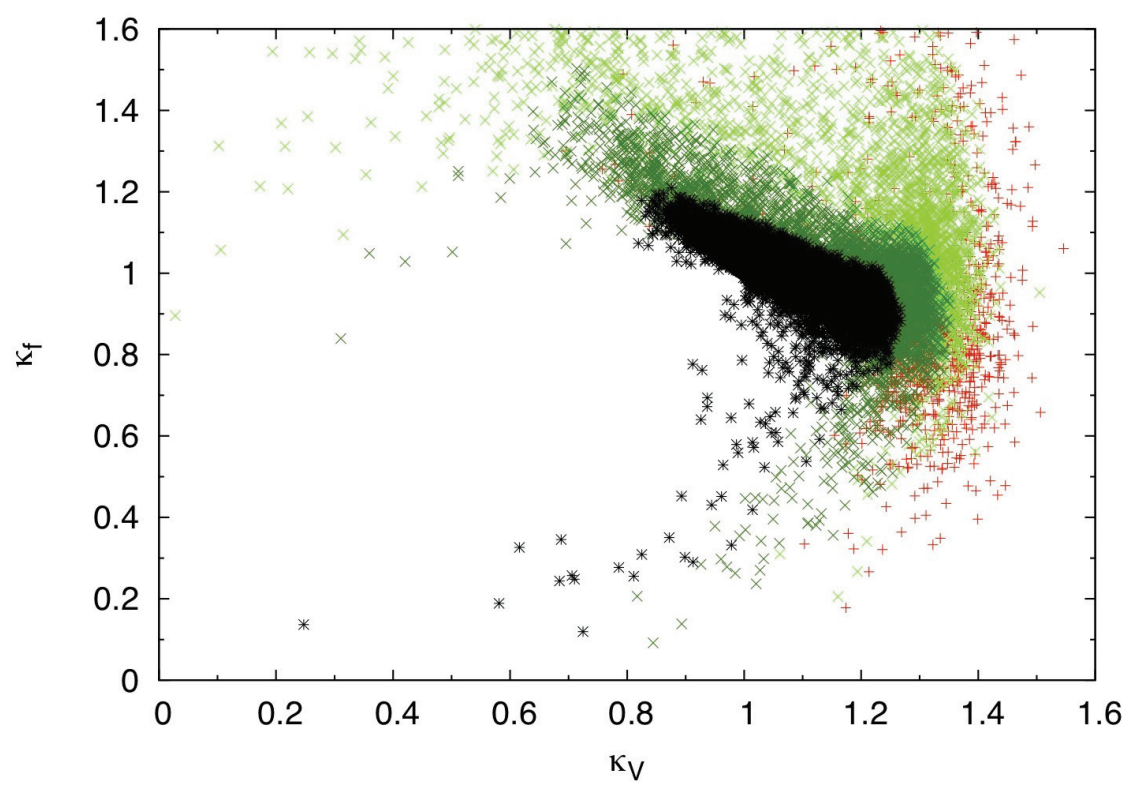

Figure 7.15: The allowed correlations between $\kappa_{V}$ and $\kappa_{f}$ after applying the constraints from $\mathrm{BR}(b \rightarrow s \gamma)$ and the $S$ parameter. The colour codes are the same as in Fig. 7.12.

simultaneous enhancement of all Higgs couplings to fermions and vector bosons has the potential to hide the BSM effects in the current experimental data if it is appropriately balanced by an increase in the branching ratio to unobserved decay modes (see Eq. (7.6)). In other words, the Higgs coupling fits from LHC data have a flat direction [34] unless additional theory assumptions are imposed. For example, one can forbid new, unobserved Higgs decay modes [34] or require that $\kappa_{V} \leq 1$, which is a valid condition for models with scalar electroweak singlets or doublets [167]. ${ }^{13}$ The GM model provides a concrete example of a model that violates this second $\kappa_{V} \leq 1$ assumption while being consistent with other experimental constraints.

However, it is important to note that the simultaneous enhancement of $\kappa_{V}$

\footnotetext{
${ }^{13}$ The flat direction could also be restricted by constraining the total Higgs width through measurements of off-shell $g g \rightarrow h^{*} \rightarrow Z Z[168,169]$. However, the interpretation of this measurement in terms of a Higgs width constraint is itself model-dependent [170] and it is not yet clear what effect the presence of additional Higgs states will have.
} 

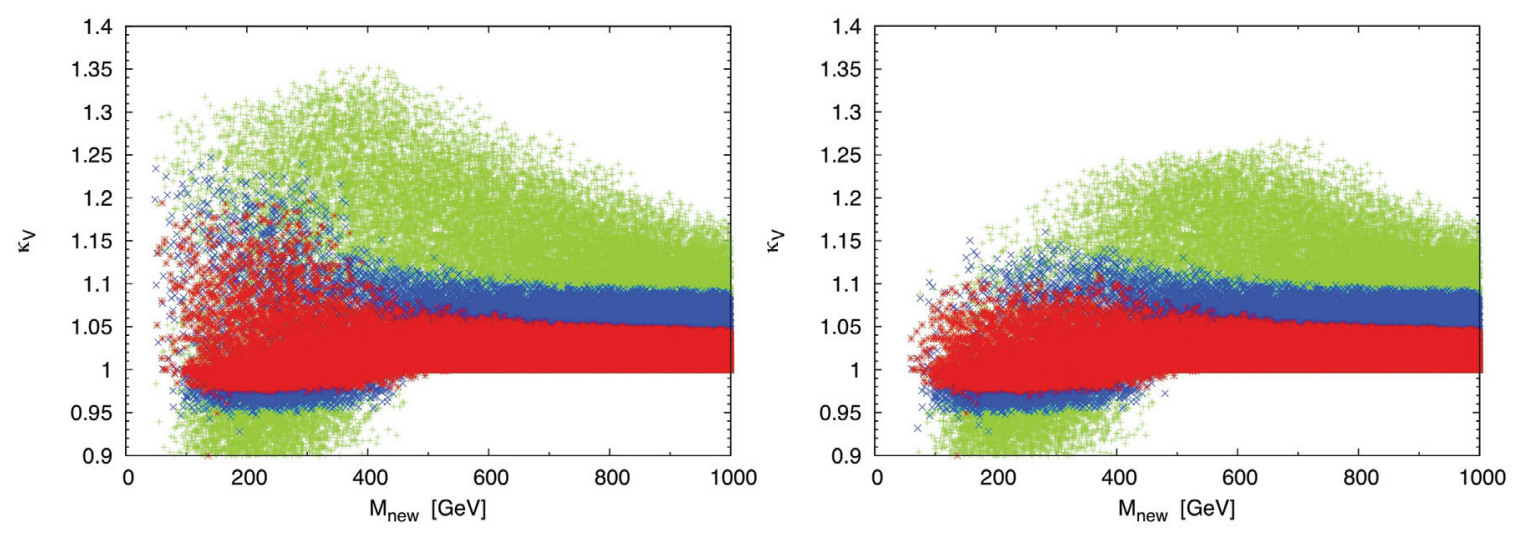

Figure 7.16: $\kappa_{V}$ as a function of the mass of the lightest new scalar, after imposing the constraint from the $S$ parameter and the best-fit (left) and experimental (right) constraint from $b \rightarrow s \gamma$. Points for which $\left|\kappa_{f} / \kappa_{V}-1\right|<5 \%$ are shown in red (medium gray), points for which $\left|\kappa_{f} / \kappa_{V}-1\right|<10 \%$ are shown in blue (dark gray), and the remaining points are shown in green (light gray).

and $\kappa_{f}$ occurs only when the new scalars are relatively light. This is illustrated in Fig. 7.16, where we plot $\kappa_{V}$ as a function of the mass of the lightest new scalar, for $\kappa_{f}$ within $5 \%$ (red) or $10 \%$ (blue) of $\kappa_{V}$. The remaining points are shown in green. Under the best-fit $b \rightarrow s \gamma$ constraint and with the requirement that $\kappa_{f}$ be within $5 \%$ of $\kappa_{V}$, an $18 \%$ enhancement of these couplings is possible only when at least one of the BSM scalar masses is below about $375 \mathrm{GeV}$. As this mass scale is well within the reach of the LHC, we expect that direct searches will be able to significantly constrain this coupling-enhancement mechanism.

\subsection{Constraints from direct collider searches}

The scalars of the GM model are subject to constraints from a variety of collider measurements and direct experimental searches for BSM physics. As usual, the most general bound arises from the LEP measurement discussed in Sec. 3.2.1. Since $H_{3}^{ \pm}$, 
$H_{5}^{ \pm}$, and $H_{5}^{ \pm \pm}$couple to the $Z$ boson they are constrained to be heavier than $45 \mathrm{GeV}$.

One of the clearest signatures for the model is the existence of the doubly charged scalar $H_{5}^{ \pm \pm}$. Evidence for the existence of such a particle would clearly favour the GM model over the SM and doublet models such as the 2HDM. Collider searches for this doubly-charged scalar depend strongly on its preferred decay channels. For example, LHC searches have constrained doubly-charged scalars to be heavier than about $400 \mathrm{GeV}$ if they decay dominantly to leptons, $H^{++} \rightarrow \ell^{+} \ell^{+}$[171]. However, the GM model generally favours the $H_{5}^{++} \rightarrow W^{+} W^{+}$decay mode. As a result, Ref. [36] has placed a lower bound of $m_{5} \leq 76 \mathrm{GeV}$ on the GM custodial fiveplet masses by adapting a constraint on $H^{ \pm \pm} \rightarrow W^{ \pm} W^{ \pm} \rightarrow \mu^{ \pm} \mu^{ \pm} \nu \nu$ from like-sign dimuon search data at ATLAS.

Furthermore, in Ref. [146] Chiang et al have also applied bounds to the $H_{5}^{++} \rightarrow W^{+} W^{+}$decay scenario by recasting an ATLAS measurement [172] of the like-sign $W W j j$ cross section in $8 \mathrm{TeV}$ LHC data in the context of the GM model. In this process, the doubly charged scalar $H_{5}^{++}$is produced in the $s$-channel through vector boson fusion, and may subsequently decay back to $W^{+} W^{-}$. Their results therefore exclude an additional portion of the $\left(m_{5}, v_{\chi}\right)$ parameter space. This excluded region is triangular in shape - narrowing as $v_{\chi}$ becomes smaller - and excludes $m_{5} \simeq 120 \mathrm{GeV}$ to $610 \mathrm{GeV}$ at $v_{\chi}=65 \mathrm{GeV}$, down to $v_{\chi} \simeq 33 \mathrm{GeV}$ at $m_{5} \simeq 200 \mathrm{GeV}$. This constraint provides a nice complementarity to the theoretical and indirect experimental constraints on the $\left(m_{5}, v_{\chi}\right)$ plane shown in the right-hand panel of Fig. 7.12. The constraint from Chiang et al substantially strengthens the bound on $v_{\chi}$ for $m_{5}$ near $200 \mathrm{GeV}$, while the theoretical and indirect constraints are stronger for higher masses of the doubly-charged scalar. Similar bounds on $v_{\chi}$ have been obtained in Ref. [148] by studying various GM scalar decay processes (notably 
$\left.H_{5}^{0} \rightarrow Z Z \rightarrow 4 \ell\right)$ in the context of ATLAS results.

The ATLAS Collaboration has also recently published the results of direct searches for a charged scalar boson produced through vector boson fusion and which has a $100 \%$ branching fraction of $H^{+} \rightarrow W^{+} Z$ [173]. This study specifically considered the effect of the search on the $\left(\sin \theta_{H}, m_{5}\right)$ plane of the GM model. The results exclude $240 \leq m_{5} \leq 700 \mathrm{GeV}$ for $\sin \theta_{H}=1$, and roughly $300 \leq m_{5} \leq 740 \mathrm{GeV}$ for $\sin \theta_{H}=0.8$. The excluded range reaches lower limits of about $\sin \theta_{H} \simeq 0.6$. As this corresponds to $v_{\chi} \simeq 52 \mathrm{GeV}$, most of the region of parameter space excluded by this direct search corresponds to the region excluded by the theoretical constraints (see Sec. 7.2) and $b \rightarrow s \gamma$ constraint (see Sec. 7.4.2).

ATLAS has also released data from a search for a neutral CP-odd scalar decaying to $Z h$ [174], analogous to $H_{3}^{0}$ in the GM model. As the results were interpreted in the context of a $2 \mathrm{HDM}$, a dedicated study would be required to precisely apply these results to the GM model. However, a naive translation of the effects of this search on the $2 \mathrm{HDM}$ parameter space suggest that these results may also roughly constrain $v_{\chi} \leq 71 \mathrm{GeV}$ for $240 \leq m_{3} \leq 350 \mathrm{GeV}$.

\subsection{Loop-decays in the Georgi-Machacek model}

As discussed in Sec. 7.5, many of the most promising avenues for further investigation of the GM model phenomenology will require model-specific collider studies of the direct search results and data being produced at the LHC. The branching ratios of particle decay modes are important in order to correctly interpret rates measured at these collider experiments. An accurate evaluation of the branching ratios for the GM scalars requires a calculation of the decays through electroweak loop processes 
to $\gamma \gamma, Z \gamma$, and $W \gamma$. These loop decay modes will be particularly important for the fermiophobic scalars $H_{5}^{0}, H_{5}^{ \pm}$and $H_{5}^{ \pm \pm}$.

The general formula for the decay width $\Gamma\left(H_{i} \rightarrow V \gamma\right)$ of a parent scalar $H_{i}$ to daughter vector bosons $V \gamma(V=\gamma, Z, W)$ is [175]

$$
\Gamma\left(H_{i} \rightarrow V \gamma\right)=\frac{m_{H_{i}}^{3}}{32 \pi \eta_{V \gamma}}\left[1-\frac{m_{V}^{2}}{m_{H_{i}}^{2}}\right]^{3}\left(\left|A_{t o t}^{V \gamma}\right|^{2}+\left|\tilde{A}_{t o t}^{V \gamma}\right|^{2}\right)
$$

where $\eta_{V \gamma}$ is a symmetry factor accounting for identical particles in the final state, i.e. $\eta_{\gamma \gamma}=2$ and all other $\eta_{V \gamma}=1$. The form factors $A_{t o t}$ and $\tilde{A}_{t o t}$ are the components of the amplitude $\mathcal{M}$ defined by

$$
\mathcal{M}=\left[\left(g^{\mu \nu} q_{\gamma} \cdot q_{V}-q_{\gamma}^{\mu} q_{V}^{\nu}\right) A_{t o t}+i \epsilon^{\mu \nu \alpha \beta} q_{\gamma \alpha} q_{V \beta} \tilde{A}_{t o t}\right] \varepsilon_{\mu}^{*}\left(q_{V}\right) \varepsilon_{\nu}^{*}\left(q_{\gamma}\right)
$$

where $q_{\gamma}$ and $q_{V}$ are the respective outgoing four-momenta of the photon and the vector boson $V$. For CP-even neutral decays to $\gamma \gamma$ and $Z \gamma \tilde{A}_{t o t}=0$, while for CP-odd neutral scalar decays, $A_{t o t}=0$.

Due to the custodial symmetry of the GM model, in the case of the neutral singlet states $h$ and $H$ only a single species of particle may propagate in the loop decays to $\gamma \gamma$ and $Z \gamma$, and so the amplitudes are described by the usual formulas in Sec. 3.2.4. However, in the case of the $H_{3}$ and $H_{5}$ scalars, additional diagrams may contribute to the loop decays in which more than one type of particle may propagate within a single loop diagram. As a result, a calculation of these amplitudes requires new loop functions not present in the SM.

In calculating these contributions, we have made use of the method of Pich and Ilisie [175]; noting that the $A_{t o t}$ contribution of the matrix element is proportional to $\left(g^{\mu \nu} q_{\gamma} \cdot q_{V}-q_{\gamma}^{\mu} q_{V}^{\nu}\right)$, as seen in Eq. (7.124), one may neglect all matrix element terms proportional to $g^{\mu \nu}$ and calculate $A_{\text {tot }}$ by considering only the contributions to $\mathcal{M}$ 


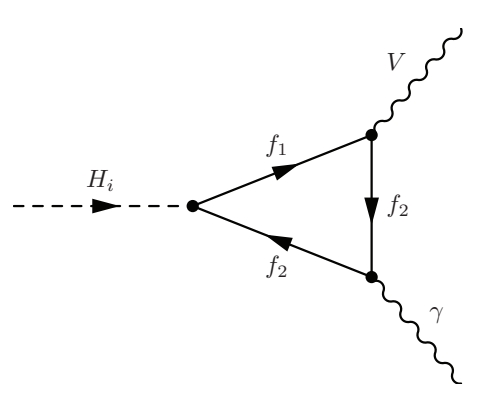

(a)

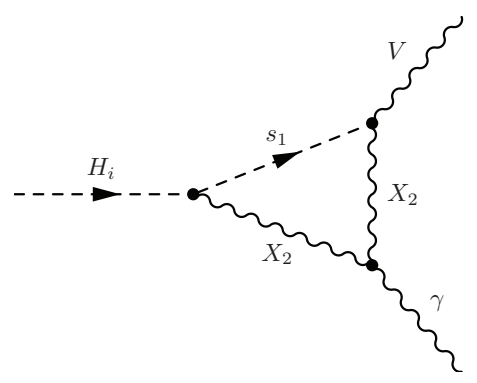

(d)

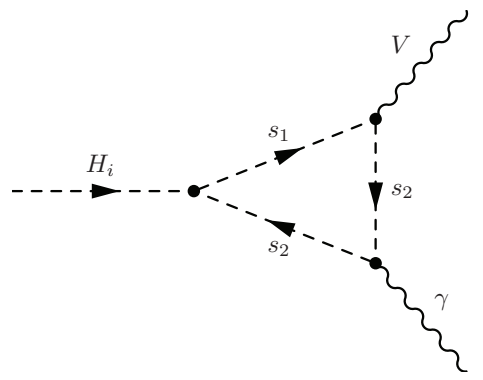

(b)

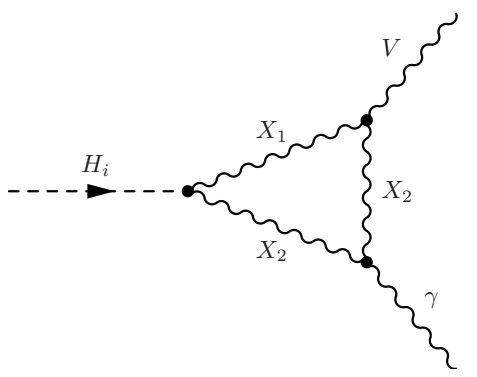

(e)

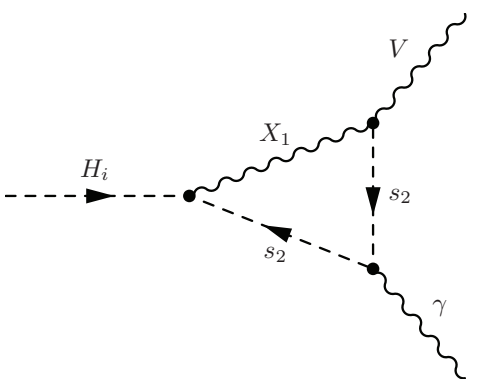

(c)

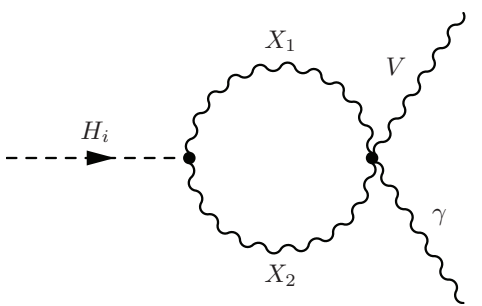

(f)

Figure 7.17: Diagrams contributing to $h \rightarrow V \gamma$.

that are proportional to $q_{\gamma}^{\mu} q_{V}^{\nu}$. In this method, the only new diagrams which must be considered are illustrated generically in Fig. 7.17.

One can see that the new diagrams fall into the following categories based on the particles propagating in the loop:

- Fermion loops: two species of fermion $f_{1}$ and $f_{2}$ ( $f$ diagrams, Fig. 7.17a),

- Scalar loops: two species of scalar $s_{1}$ and $s_{2}$ (sss diagrams, Fig. 7.17b),

- Scalar-vector loops: two scalars $s_{2}$ and one vector boson $X_{1}$ (ssX, Fig. 7.17c),

- Vector-scalar loops: two vector bosons $X_{2}$ and one scalar $s_{1}(s X X$, Fig. 7.17d),

- Vector loops: two species of vector boson $X_{1}$ and $X_{2}$ (XXX, Figs. 7.17e-7.17f). 
One will note that in following the Pich and Ilisie method only triangle diagrams are relevant for the first four categories of diagrams, as seen in Figs. 7.17a-7.17d. However, in the case of the purely gauge boson loops the two-point loop diagram of Fig. 7.17f also contributes. In general, when distinguishing between two species of particles propagating in the loop we will label the particle propagating between the incoming scalar and the outgoing vector boson $V$ with a subscript 1 , while the particle propagating through the remainder of the loop (i.e. through the photon coupling) will be labeled with a 2 .

The result for the scalar diagram (Fig. 7.17b) has previously been presented in Ref. [175] for the Aligned 2HDM in the limit that $H_{i}=s_{2}$, as has the result for the vector-scalar diagram (Fig. 7.17d) in the limit that $V \rightarrow W^{+}, X \rightarrow W^{-}$. However, to our knowledge the results for the scalar-vector and vector diagrams (Figs. 7.17c and 7.17e-7.17f) have not previously appeared in the literature.

\subsubsection{Scalar decays to photons}

The neutral GM scalars $h, H, H_{3}^{0}$, and $H_{5}^{0}$ may all decay to two photons. Due to the diagonality of the photon couplings, the loops in these decays may contain only one species of propagator. As a result, the contributions from scalars, fermions, and vector

bosons propagating in these loops are described by the usual $A_{S, F, W}^{H_{i} \gamma \gamma}$ form factors ${ }^{14}$ given in Eqs. (3.59-3.61). As both the $h$ and $H$ scalars couple to all charged particles, $A_{t o t}^{h \gamma \gamma}$ is defined identically to the usual Eq. (3.58) and $A_{t o t}^{H \gamma \gamma}$ similarly becomes

$$
A_{t o t}^{H \gamma \gamma}=\frac{\alpha_{\mathrm{em}} g}{4 \pi m_{W}}\left(\beta_{f}^{H} A_{F}^{H \gamma \gamma}+\beta_{W}^{H} A_{W}^{H \gamma \gamma}+A_{S}^{H \gamma \gamma}\right)
$$

where $\alpha_{\mathrm{em}}=e^{2} / 4 \pi$ is the fine structure constant. The beta factors are defined in

\footnotetext{
${ }^{14}$ Note that any properties of the Higgs $h$ must be replaced with those of the appropriate parent scalar $H_{i}$, i.e. the mass $m_{h} \rightarrow m_{H_{i}}$ and couplings $C_{h j k} \rightarrow C_{H_{i} j k}$.
} 
analogy to $\kappa_{f, V}$ as

$$
\beta_{f}^{H_{i}}=-\frac{C_{H_{i} f f^{*}} v}{m_{f}} \quad \beta_{W}^{H_{i}}=\frac{C_{H_{i} W W^{*}} e^{2}}{g m_{W}}=\frac{2 s_{W}^{2} C_{H_{i} W W^{*}}}{v},
$$

for a propagating fermion $f$ or $W$ boson respectively.

In the case of the $H_{5}^{0} \rightarrow \gamma \gamma$ decay, there is no loop contribution from fermions, as the $H_{5}$ states are fermiophobic. As a result, the form factor takes the form

$$
A_{t o t}^{H_{5}^{0} \gamma \gamma}=\frac{\alpha_{\mathrm{em}} g}{4 \pi m_{W}}\left(\beta_{W}^{H_{5}^{0}} A_{W}^{H_{5}^{0} \gamma \gamma}+A_{S}^{H_{5}^{0} \gamma \gamma}\right)
$$

where the scalar and vector boson components $A_{S}^{H_{5}^{0} \gamma \gamma}$ and $A_{W}^{H_{5}^{0} \gamma \gamma}$ are respectively identical in form to Eqs. (3.59) and (3.60).

In the case of $H_{3}^{0}$, only fermions may propagate in the loop. As $H_{3}^{0}$ is a pseudo-scalar, this contribution is not defined by Eq. (3.61), but is instead given by ${ }^{15}$

$$
\tilde{A}_{t o t}^{H_{3}^{0} \gamma \gamma}=\frac{\alpha_{\mathrm{em}} g}{4 \pi m_{W}}\left(\beta_{f}^{H_{3}^{0}} \tilde{A}_{A}^{H_{3}^{0} \gamma \gamma}\right)
$$

with $A_{t o t}=0$ and where

$$
\tilde{A}_{A}^{H_{i} \gamma \gamma}=-2 \sum_{f} N_{c, f} Q_{f}^{2} \tau_{f} f\left(\tau_{f}\right)
$$

\subsubsection{Scalar decays to a photon and a $Z$}

All of the neutral scalars in the GM model may also decay to $Z \gamma$. Similarly to the $\gamma \gamma$ decay, only a single species of scalar, fermion or vector boson may propagate in the loops of $h$ or $H$ decays. Therefore the form factor $A_{t o t}^{h Z \gamma}$ is defined identically to Eq. (3.69), and $A_{t o t}^{H Z \gamma}$ is similarly given by

$$
A_{t o t}^{H Z \gamma}=-\frac{\alpha_{\mathrm{em}} g}{4 \pi m_{W}}\left(\beta_{f}^{H} A_{F}^{H Z \gamma}+\beta_{W}^{H} A_{W}^{H Z \gamma}+A_{S}^{H Z \gamma}\right)
$$

\footnotetext{
${ }^{15}$ In Appendix A.3.3 we have defined the pseudoscalar couplings $C_{H_{3}^{0} \bar{f} f}$ to be consistent with $\beta_{f}^{H_{i}}$.
} 
where $\beta_{f, W}$ are defined in Eq. (7.126).

In the case of $H_{5}^{0} \rightarrow Z \gamma$ decay, there will be contributions from single-species loops of scalars and vector bosons which will be described by the same formulas $A_{S, W}^{H_{5}^{0} Z_{\gamma}}$ defined in Eqs. (3.70) and (3.71). As $H_{5}^{0}$ is fermiophobic, there is no contribution from fermions. However, there will be additional contributing diagrams: scalar-vector loops with two $H_{5}^{ \pm}$and one $W^{ \pm}$(see Fig. 7.17c), and vector-scalar loops with two $W^{ \pm} \mathrm{s}$ and one $H_{5}^{ \pm}$(see Fig. $7.17 \mathrm{~d}$ ). As such, the total form factor becomes

$$
A_{t o t}^{H_{5}^{0} Z \gamma}=-\frac{\alpha_{\mathrm{em}} g}{4 \pi m_{W}}\left(\beta_{W}^{H_{5}^{0}} A_{W}^{H_{5}^{0} Z \gamma}+A_{S}^{H_{5}^{0} Z \gamma}\right)+2\left(A_{H_{5}^{+} W^{+} W^{+}}^{H_{0}^{0} Z_{\gamma}}+A_{H_{5}^{+} H_{5}^{+} W^{+}}^{H_{0}^{0} Z_{\gamma}}\right)
$$

Here the factor of two over the last two terms accounts for the diagrams of opposite sign, $A_{H_{5}^{-} W^{-} W^{-}}^{H_{0}^{0} Z_{\gamma}}$ and $A_{H_{5}^{-} H_{5}^{-} W^{-}}^{H^{0}{ }^{16}}$ For a parent scalar $H_{i}$, daughter vector bosons $V \gamma$, and propagating scalar $s$ and vector boson $X$, the vector-scalar contribution $A_{H_{5} W W}^{H_{5}^{0} Z \gamma}$ is given by

$$
A_{s X X}^{H_{i} V \gamma}=\frac{\alpha_{\mathrm{em}}^{2} Q_{X} C_{X H_{i} s^{*}} C_{s X^{*} V^{*}}}{2 m_{X}^{2}} \int_{0}^{1} d x \int_{0}^{1} d z \frac{I_{s X X}^{H_{i} V \gamma}}{\Delta_{s X X}}
$$

where $C_{X H_{i} s^{*}}$ and $C_{s X^{*} V^{*}}$ are defined without factors of $e$ (see Appendix A.3), ${ }^{17}$

$$
\begin{aligned}
\Delta_{s X X}= & -x(1-x) m_{V}^{2}+(1-x) m_{s}^{2}+x m_{X}^{2}-x z(1-x)\left(m_{H_{i}}^{2}-m_{V}^{2}\right), \text { and } \\
I_{s X X}^{H_{i} V \gamma}= & {\left[\frac{2}{3} x^{4}(z-2)(z-1)+\frac{4}{3} x^{3}\left(2 z^{2}-3 z+1\right)+x^{2}(z-1)\right] m_{V}^{2} } \\
& +\left[-\frac{2}{3} x^{4} z(1+z)+\frac{8}{3} x^{3} z(2-z)-3 x^{2} z\right] m_{H_{i}}^{2}+\left[-\frac{2}{3} x^{3}(1+4 z)+x^{2}(6 z-1)\right] m_{s}^{2} \\
& +\left[\frac{2}{3} x^{3}(4 z+1)+3 x^{2}(3-2 z)\right] m_{X}^{2} .
\end{aligned}
$$

Eq. 7.132 is consistent with the Ref. [175] result when $V=W^{+}, X=W^{-} .{ }^{18}$

\footnotetext{
${ }^{16}$ Unlike the formulas for $A_{S, W, f}^{H_{i} V \gamma}$, the form factors $A_{s s X}^{H_{i} V \gamma}$ and $A_{s X X}^{H_{i} V \gamma}$ defined in Eqs. (7.132-7.134) do not account for diagrams of opposite sign.

${ }^{17}$ Note that in our notation the order of the scalars in couplings of the form $C_{V s_{1} s_{2}}$ matters, as swapping $s_{1}$ and $s_{2}$ introduces an overall minus sign.

${ }^{18}$ However, $I_{s X X}$ is defined such that our integral differs from theirs by a factor of 4 .
} 
Similarly, the scalar-vector contribution $A_{H_{5} H_{5} W}^{H_{5}^{0} Z_{\gamma}}$ is described by

$$
A_{s s X}^{H_{i} V \gamma}=-\frac{\alpha_{\mathrm{em}}^{2} Q_{s} C_{X^{*} H_{i} s} C_{s^{*} X V^{*}}}{m_{X}^{2}} \int_{0}^{1} d x \int_{0}^{1} d z \frac{I_{s s X}^{H_{i} V \gamma}}{\Delta_{s s X}}
$$

where $\Delta_{s s X}=-x(1-x) m_{V}^{2}+(1-x) m_{X}^{2}+x m_{s}^{2}-x z(1-x)\left(m_{H_{i}}^{2}-m_{V}^{2}\right)$, and

$$
\begin{aligned}
I_{s s X}^{H_{i} V \gamma}= & x^{2} z\left[\frac{2}{3} x^{2}(1+z)+\frac{2}{3} x(1-2 z)-1\right] m_{H_{i}}^{2}+x^{2}(z-1)\left[\frac{2}{3} x(2-x)(z-2)+1\right] m_{V}^{2} \\
& +x\left[\frac{2}{3}(z+1) x^{2}-5 x+4\right] m_{X}^{2}+x^{2}\left[-\frac{2}{3}(1+z) x+1\right] m_{s}^{2} .
\end{aligned}
$$

The form factor for the $H_{3}^{0} \rightarrow Z \gamma$ decay contains only contributions from fermions. Because $H_{3}^{0}$ is a pseudo-scalar, this fermion term contributes to $\tilde{A}_{t o t}$ while $A_{t o t}=0$. The anti-symmetric form factor takes the form

$$
\tilde{A}_{t o t}^{H_{3}^{0} Z \gamma}=-\frac{\alpha_{\mathrm{em}} g}{4 \pi m_{W}}\left(\beta_{f}^{H_{3}^{0}} \tilde{A}_{A}^{H_{3}^{0} Z \gamma}\right)
$$

where - with $I_{2}$ from Eq. (3.76), and $\beta_{f}$ from Eq. (7.126) — we define

$$
A_{A}^{H_{i} Z \gamma}=\sum_{f} N_{c f} \frac{-2 Q_{f}\left(T_{f}^{3 L}-2 Q_{f} \sin ^{2} \theta_{W}\right)}{\sin \theta_{W} \cos \theta_{W}}\left[-I_{2}\left(\tau_{f}, \lambda_{f}\right)\right] .
$$

\subsubsection{Scalar decays to a photon and a $W$}

The charged scalars $H_{3}^{+}$and $H_{5}^{+}$may also decay through loops to $W^{+} \gamma$. Because $W^{+}$ is electrically charged, these loops always contain more than one species of propagating particle. The scalar-vector and vector-scalar contributions are given by the same formulas previously seen above (Eqs. (7.134)-(7.132)). Both the $H_{3}^{+}$and $H_{5}^{+}$may receive contributions from the scalar-vector $\left(A_{s s X}^{H_{i} V \gamma}\right)$ and vector-scalar $\left(A_{s X X}^{H_{i} V \gamma}\right)$ diagrams described by Eqs. (7.134) and (7.132) above, as well as from mixed scalar diagrams $\left(A_{s_{1} s_{2} s_{2}}^{H_{i} V \gamma}\right)$. Additionally, the fermiophobic $H_{5}^{+}$receives a contribution from a mixed vector diagram $\left(A_{X_{1} X_{2} X_{2}}^{H_{i} V \gamma}\right.$, see Figs. 7.17e-7.17f), while the fermiophilic $H_{3}^{+}$receives a contribution from mixed fermion diagrams $\left(A_{f}^{H_{i} V \gamma}\right.$ and $\tilde{A}_{f}^{H_{i} V \gamma}$, see Fig. 7.17a). 
The form-factor for mixed scalar diagrams is given by

$$
A_{s_{1} s_{2} s_{2}}^{H_{i} V \gamma}=-\frac{\alpha_{\mathrm{em}} Q_{s_{2}}}{\pi} C_{H_{i} s_{1}^{*} s_{2}} C_{V^{*} s_{1} s_{2}^{*}} \int_{0}^{1} d x \int_{0}^{1} d z \frac{x^{2} z(1-x)}{\Delta_{s_{1} s_{2} s_{2}}}
$$

where $\Delta_{s_{1} s_{2} s_{2}}=m_{V}^{2} x(x-1)+m_{s_{1}}^{2}(1-x)+m_{s_{2}}^{2} x+\left(m_{V}^{2}-m_{H_{i}}^{2}\right) x z(1-x)$, The $A_{s_{1} s_{2} s_{2}}^{H_{i} V \gamma}$ result is consistent with that of Ref. [175]. We have further checked that it is consistent with Eq. (3.70) in the limit that $m_{s_{1}}=m_{s_{2}} \cdot{ }^{19}$

The contribution of the vector loops to the form factor $A_{t o t}$ is described by

$$
A_{X_{1} X_{2} X_{2}}^{H_{i} V \gamma}=\frac{\alpha_{\mathrm{em}}^{2} Q_{X_{2}} C_{X_{1} V^{*} X_{2}^{*}} C_{H_{i} X_{2} X_{1}^{*}}}{2} \int_{0}^{1} d x \int_{0}^{1} d z \frac{I_{X_{1} X_{2} X_{2}}^{H_{i} V \gamma}}{\Delta_{X_{1} X_{2} X_{2}}},
$$

where $\Delta_{X_{1} X_{2} X_{2}}=m_{V}^{2} x(x-1)+m_{X_{1}}^{2}(1-x)+m_{X_{2}}^{2} x+\left(m_{V}^{2}-m_{H_{i}}^{2}\right) x z(1-x)$, and the numerator of the integrand is given by

$$
\begin{aligned}
& I_{X_{1} X_{2} X_{2}}^{H_{i} V \gamma}=\frac{x}{3 m_{X_{1}}^{2} m_{X_{2}}^{2}}\left[m_{X_{1}}^{4}(x[117-6 x(34+16 x[z-1]-29 z)-72 z]-12)\right. \\
& \quad+m_{X_{2}}^{2}\left(m_{X_{2}}^{2} x[9+6 x(z-6-16 x[z-1])]+m_{X_{1}}^{2}[36+6 x(x[40-18 z]\right. \\
& \left.\left.\left.\quad+32 x^{2}[z-1]-3[3+4 z]\right)\right]\right)+m_{H_{i}}^{2} x\left(m_{H_{i}}^{2} x[x z(2-24 x[11+5 x(z-1)-9 z] z\right. \\
& \quad-90[z-2] z)-4 z(1+9 z)]+m_{X_{2}}^{2}[x(6 z[13+2 z]-2-12 x[23+18 x(z-1) \\
& \left.\quad-16 z] z)-9 z]+m_{X_{1}}^{2}[57 z+x(2+12 x[39+18 x(z-1)-32 z] z+6 z[26 z-51])]\right) \\
& \quad+m_{V}^{2}\left(m_{X_{2}}^{2} x[9 z-12+x(64+12 x[23+18 x(z-1)-16 z](z-1)-2 z[29+6 z])]\right. \\
& \quad+m_{V}^{2} x\left[3-3 z-2 x(z-1)^{2}\left(20+60 x^{3}[z-1]-12 x^{2}[9 z-11]+x[45 z-88]\right)\right] \\
& \quad+m_{X_{1}}^{2}[x(72-57 z+x[(454-156 z) z-304-12 x(39+18 x[z-1]-32 z)(z-1)]) \\
& \quad-12]+m_{H_{i}}^{2} x\left[3 z+x\left(2+z[76 z-78]+4 x[z-1]\left[1+\left(132 x-60 x^{2}-89\right) z\right.\right.\right. \\
& \left.\left.\left.\left.\left.\quad+3\left(15-36 x+20 x^{2}\right) z^{2}\right]\right)\right]\right)\right] .
\end{aligned}
$$

\footnotetext{
${ }^{19}$ Note that there is a factor of 2 difference between Eq. (7.138) in this limit and Eq. (3.70) as the latter accounts for the crossed diagram that exists when $m_{s_{1}}=m_{s_{2}}$ (i.e. the diagram with propagators of opposite sign).
} 
For the appropriate combination of SM gauge bosons, the coupling $C_{X_{1} V^{*} X_{2}^{*}}=$ $C_{Z W^{-} W^{+}}=c_{W} / s_{W}$. We have checked that this formula reduces to Eq. (3.71) with the appropriate choice of gauge bosons, masses, and couplings. ${ }^{20}$

The contributions from loops with mixed fermion species (see Fig. 7.17a) are the only diagrams to contribute to both the $A$ and $\tilde{A}$ form factors. These contributions may be adapted from those in Ref. [175] — where they were derived for the Aligned $2 \mathrm{HDM}-$ with a mixing angle translation of $\cot \beta \rightarrow \tan \theta$,

$$
\begin{aligned}
A_{f}^{H_{3}^{+} W \gamma}= & \frac{\alpha_{\mathrm{em}} N_{c}\left|V_{t b}\right|^{2}}{2 \pi v s_{W}} \tan \theta_{H} \int_{0}^{1} d x \int_{0}^{1} d z\left[Q_{t} x+Q_{b}(1-x)\right] \\
& \times \frac{-m_{t}^{2} x(2 x z-2 z+1)+m_{b}^{2}(1-x)(1-2 x z)}{m_{W}^{2} x(x-1)+m_{b}^{2}(1-x)+m_{t}^{2} x+\left(m_{W}^{2}-m_{3}^{2}\right) x z(1-x)}, \\
\tilde{A}_{f}^{H_{3}^{+} W \gamma}= & \frac{\alpha_{\mathrm{em}} N_{c}\left|V_{t b}\right|^{2}}{2 \pi v s_{W}} \tan \theta_{H} \int_{0}^{1} d x \int_{0}^{1} d z\left[Q_{t} x+Q_{b}(1-x)\right] \\
& \times \frac{m_{t}^{2} x+m_{b}^{2}(1-x)}{m_{W}^{2} x(x-1)+m_{b}^{2}(1-x)+m_{t}^{2} x+\left(m_{W}^{2}-m_{3}^{2}\right) x z(1-x)} .
\end{aligned}
$$

Note that these equations account for contributions from propagating particles $t b$, and from propagating anti-particles $\bar{b} \bar{t}$.

The full form factors $A$ for the $H_{3}^{+} \rightarrow W^{+} \gamma$ and $H_{5}^{+} \rightarrow W^{+} \gamma$ decays are therefore given by

$$
\begin{aligned}
& A_{t o t}^{H_{5}^{+} W \gamma}=\sum A_{s_{1} s_{2} s_{2}}^{H_{+}^{+} W \gamma}+\sum A_{s s X}^{H_{5}^{+} W \gamma}+\sum A_{s X X}^{H_{5}^{+} W \gamma}+A_{Z W W}^{H_{5}^{+} W \gamma} \\
& A_{t o t}^{H_{3}^{+} W \gamma}=\sum A_{s_{1} s_{2} s_{2}}^{H_{3}^{+} W \gamma}+\sum A_{s s X}^{H_{3}^{+} W \gamma}+\sum A_{s X X}^{H_{3}^{+} W \gamma}+A_{f}^{H_{3}^{+} W \gamma},
\end{aligned}
$$

while $\tilde{A}_{t o t}^{H_{5}^{+} W \gamma}=0$ and $\tilde{A}_{t o t}^{H_{3}^{+} W \gamma}=\tilde{A}_{f}^{H_{3}^{+} W \gamma}$. The possible propagating states that are included in each sum for a given parent $H_{i}(i=3,5)$ are tabulated in Table 7.7.

\footnotetext{
${ }^{20}$ Note that there is a factor of 2 difference between Eq. (7.139) in this limit and Eq. (3.71) as the latter accounts for the additional crossed diagram.
} 
Table 7.7: Combinations of propagating particles for the $W^{+} \gamma$ decay calculations.

\begin{tabular}{cccc}
\hline \hline Parent & $A^{H_{i}^{+} W \gamma}$ & Equation & Propagating $p_{1} p_{2}$ \\
\hline$H_{3}^{+}$ & $s_{1} s_{2} s_{2}$ & 7.138 & $h H_{3}^{+*}, H H_{3}^{+*}, H_{5}^{0} H_{3}^{+*}, H_{3}^{0} H_{5}^{+*}, H_{5}^{++} H_{3}^{+}, H_{3}^{+*} H_{5}^{++*}$ \\
& $s s X$ & 7.134 & $Z H_{5}^{-}, W^{-} H_{5}^{--}$ \\
& $s X X$ & 7.132 & $h W^{-}, H W^{-}, H_{5}^{0} W^{-}, H_{5}^{++} W^{+}$ \\
& $f$ & $7.141-7.142$ & $\bar{b} \bar{t}, t b$ \\
\hline \hline$H_{5}^{+}$ & $s_{1} s_{2} s_{2}$ & 7.138 & $H_{3}^{0} H_{3}^{+*}, H_{5}^{0} H_{5}^{+*}, H_{5}^{+*} H_{5}^{++*}, H_{5}^{++} H_{5}^{+}$ \\
& $s s X$ & 7.134 & $Z H_{5}^{+*}, W^{-} H_{5}^{++*}$ \\
& $s X X$ & 7.132 & $H_{5}^{0} W^{-}, H_{5}^{++} W^{+}$ \\
& $X_{1} X_{2} X_{2}$ & 7.139 & $Z W^{-}$ \\
\hline \hline
\end{tabular}

\subsubsection{Decay branching ratios of the $H_{3}$ and $H_{5}$ scalars}

As the $H_{5}$ scalars are fermiophobic, they will decay to gauge bosons when lighter scalars are not kinematically available. The dominant decay modes will generally be to $W W, Z Z$, and $W Z$. However, electroweak loop decays to $\gamma \gamma, Z \gamma$ and $W \gamma$ will be important for the $H_{5}^{0}$ and $H_{5}^{+}$scalars $^{21}$ at low energies where $Z Z, W W$ and $W Z$ decays are off-shell. In general, the electroweak loop decay modes are not as important for the decays of the $H_{3}^{0}$ and $H_{3}^{ \pm}$scalars; they decay dominantly to fermions and $Z h$ or $W^{+} h$, and the $H_{3}^{0}$ pseudoscalar may also decay through a strongly-coupled loop to gluons. However, the electroweak loop decays may contribute more substantially in cases where the couplings to fermions are suppressed.

Figure 7.18 illustrates the branching ratios for the scalars $H_{3}^{0}, H_{3}^{+}, H_{5}^{0}$ and $H_{5}^{+}$. The starting benchmark point used for these plots is the same as that considered in the decoupling Case A of Sec. 7.3; it corresponds to the potential parameters $\lambda_{2,3,4,5}=0.1$ and $M_{1,2}=100 \mathrm{GeV}$, with a mass scale of $\mu_{3}^{2}=(500 \mathrm{GeV})^{2} \cdot{ }^{22}$ This choice

\footnotetext{
${ }^{21}$ Due to charge conservation, the $H_{5}^{++}+$scalar state will decay dominantly to $W^{+} W^{+}$when lighter scalar states are kinematically unavailable.

${ }^{22}$ The default parameter point used in the GMCalc program uses the same $\lambda_{2,3,4,5}$ and $M_{1,2}$ values,
} 


\begin{tabular}{cccccc}
\hline$m_{h}$ & $m_{H}$ & $m_{3}$ & $m_{5}$ & $\alpha$ & $\sin \theta_{H}$ \\
\hline 125 & 501 & 507 & 518 & -0.0886 & 0.0685 \\
\hline
\end{tabular}

Table 7.8: Masses (in GeV) and mixing angles for the Fig. 7.18 benchmark point.

of parameters corresponds to the masses and mixing angles outlined in Table 7.8. In producing these plots, we have allowed the mass of the parent particle - either $m_{3}$ or $m_{5}$ — to vary while keeping all other masses and mixing angles fixed. Furthermore, we set the parameters $M_{1}$ and $M_{2}$ to vary with $m_{3}$ and $m_{5}$ such that all of the particle couplings remain fixed at their original benchmark values. As such, Figure 7.18 demonstrates the variance of the loop decay modes with the decay kinematics. The formulas for the competing decay modes can be found in Appendix B.

As one would expect, the electroweak loop decays dominate for $H_{5}^{0, \pm}$ when the mass $m_{5}$ is relatively light. In the benchmark case, the $H_{5}^{0}$ decay to $\gamma \gamma$ dominates up to masses of $m_{5} \simeq 140 \mathrm{GeV}$, while $Z \gamma$ accounts for up to $5 \%$ of the decays over a comparable region (see the top right-hand panel). Similarly, the $H_{5}^{+}$loop decay to $W^{+} \gamma$ dominates up to masses of $m_{5} \leq 125 \mathrm{GeV}$ (bottom right-hand panel).

In comparison, the electroweak loop decay modes are negligible for the $H_{3}^{0}$ decays (top left-hand panel), which are dominated by decays to fermions and $Z h$ with a small contribution from gluon decay (as expected). Because $H_{3}^{0}$ is CP-odd, only fermions may propagate in the loops of the $\gamma \gamma$ and $Z \gamma$ processes. These loop decay rates are controlled by the same $H_{3}^{0} \bar{f} f$ couplings as the decays to fermions and gluons, and have very similar structure with respect to the production of fermions. In fact, above the threshold for decays to the top quark, the ratios of $\Gamma\left(H_{3}^{0} \rightarrow \gamma \gamma\right)$ and $\Gamma\left(H_{3}^{0} \rightarrow Z \gamma\right)$ to $\Gamma\left(H_{3}^{0} \rightarrow \bar{f} f\right)$ each approach a constant value as the parent mass $m_{3}$ but a different mass scale of $\mu_{3}^{2}=(300 \mathrm{GeV})^{2}[166]$. 

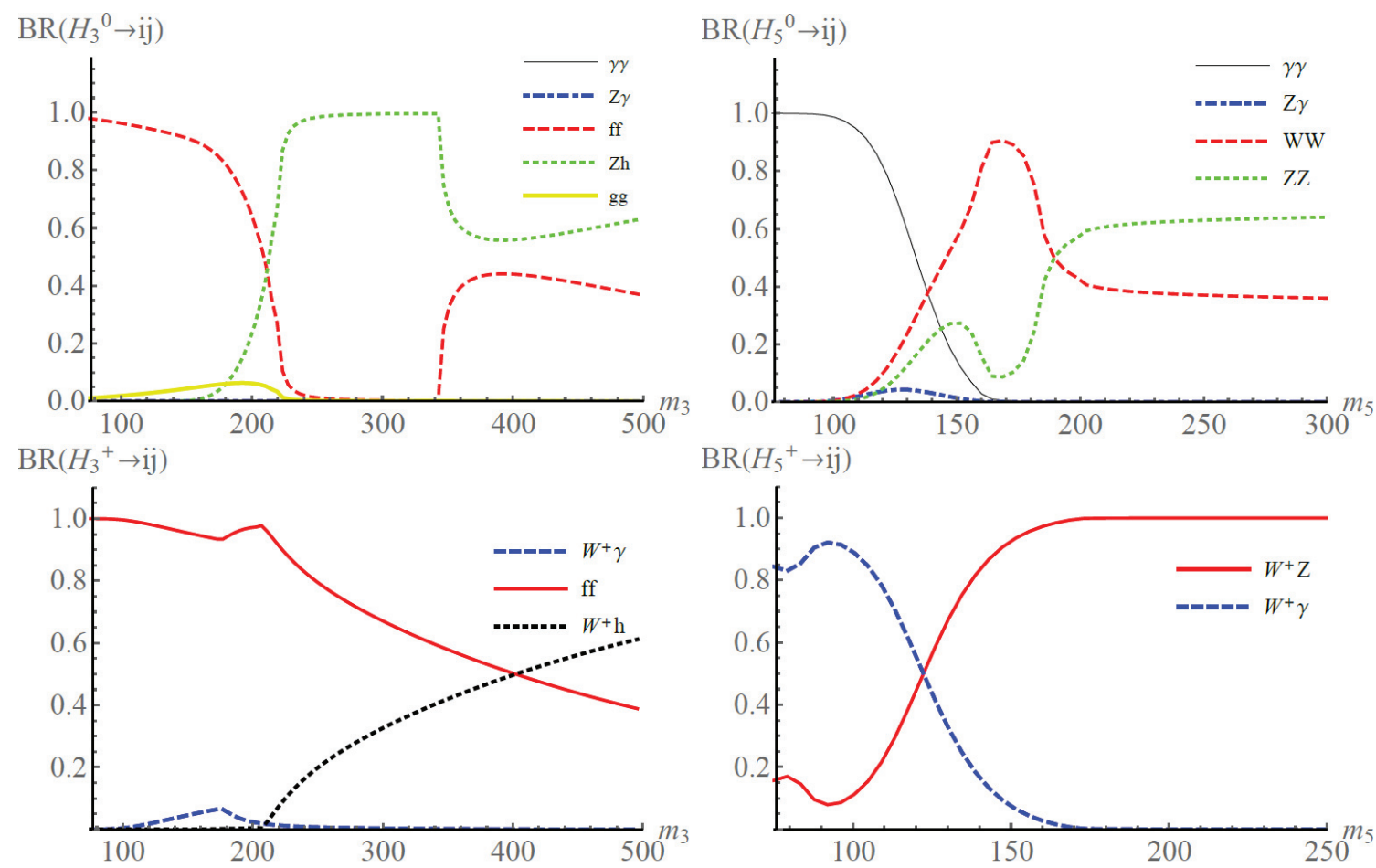

Figure 7.18: Branching ratios as functions of the mass of the parent particle. All particle couplings are held constant.

increases. $^{23}$ As the loop decays to $\gamma \gamma$ and $Z \gamma$ are additionally suppressed by extra couplings and loop factors, they will therefore always be negligible in comparison with the dominant tree-level $H_{3}^{0} \rightarrow \bar{f} f$ decay mode.

However, in the case of $H_{3}^{+}$decay the $W^{+} \gamma$ mode contributes up to $7 \%$ for parent masses $m_{3} \leq 200 \mathrm{GeV}$ in the benchmark case (see the lower left panel of Fig. 7.18). This is largely due to the fact that our chosen benchmark point corresponds to a small value of $\sin \theta_{H}=0.0685$, as seen in Table 7.8 ; in cases where $\theta_{H}$ is larger, the $W^{+} \gamma$ branching ratio would be negligible in comparison. Small values of $\sin \theta_{H}$ correspond to a suppression of the $H_{3}^{0, \pm} f_{1} \bar{f}_{2}$ couplings, which are proportional to $\tan \theta_{H}$. Unlike the $H_{3}^{0}$ case, the $H_{3}^{+}$electroweak loop decays receive contributions from

\footnotetext{
${ }^{23}$ Similarly, as seen in Appendix B, the loop decay to gluons is given by the same $\gamma \gamma$ formula scaled by $9 \alpha_{s}^{2} / 16 \alpha_{\mathrm{em}}^{2}>1$, and so $\Gamma\left(H_{3}^{0} \rightarrow \gamma \gamma\right) \leq \Gamma\left(H_{3}^{0} \rightarrow g g\right) \leq \Gamma\left(H_{3}^{0} \rightarrow \bar{f} f\right)$.
} 
diagrams with propagating scalars and vector bosons that need not be suppressed as $\sin \theta_{H}$ becomes small. As a result, a suppression of the fermion couplings can prevent the $H_{3}^{+} \rightarrow f_{1} \bar{f}_{2}$ decay from completely dominating the $W^{+} \gamma$ decay mode, as seen in the benchmark case.

As demonstrated by Figure 7.18, the loop processes $\gamma \gamma, Z \gamma$ and $W^{+} \gamma$ can play an important role in the decays of the GM triplet and fiveplet scalars $H_{3,5}$, depending on the decay kinematics of the model under consideration. As such, a clear understanding of these decay processes will be important for future GM collider studies, and a full parameter scan of the branching ratios merits further investigation. 


\section{Chapter 8}

\section{Conclusions and Summary}

Throughout this thesis, we have considered theoretical and experimental constraints that may be placed upon models which extend the Standard Model (SM) by one or more electroweak $S U(2)_{L}$ scalar multiplets that are larger than doublets $(n \geq$ 3). In Chapter 4 we determined the maximum isospin $T$ (or size $n=2 T+1$ ) and hypercharge $Y$ permitted to such multiplets by applying the limits of tree-level perturbative unitarity to the electroweak interactions of the beyond-the-SM (BSM) scalars with vector bosons. We concluded that an $S U(2)_{L}$ scalar multiplet may have a maximum isospin of $T=7 / 2(T=4)$ if the multiplet is complex (real). We further found that the hypercharge may have a maximum value of $19.8 / n^{1 / 4}$.

Throughout Chapters 5 and 6 we considered the extension of the SM by a single $S U(2)_{L}$ scalar multiplet larger than a quartet $(n \geq 5)$. In Chp. 5 we considered a general class of these models that preserve a $U(1)$ symmetry in the scalar potential, while in Chp. 6 we considered a $Z_{2}$ symmetric class of models. In these extensions the BSM multiplet does not acquire a vacuum expectation value, and the neutral scalar in the multiplet is required to be stable in order to provide a dark matter candidate. 
We applied constraints to these models using tree-level partial-wave unitarity, the oblique parameters, $h \rightarrow \gamma \gamma$, the observed thermal relic density of dark matter, and direct searches for dark matter.

After the application of these constraints, we concluded that three of the four possible $U(1)$-symmetric models — where the multiplet size $n=6,7,8$ - are completely excluded by experimental data, as the $Z$-exchange contribution to the nucleon scattering cross section of the dark matter candidate is too large to be consistent with the current limits from direct-detection experiments. However, the fourth $n=5 U(1)$ model may avoid these bounds and remain viable due to an additional operator in the scalar potential that permits the neutral scalar to decay. This $n=5$ model merits further investigation in the future.

In the $Z_{2}$-symmetric case, the breaking of the $U(1)$ symmetry down to $Z_{2}$ induces mixing in the scalar sector. As a result, the $Z$-exchange contribution to the nucleon scattering cross section becomes kinematically unfavourable. This saves the $Z_{2}$ models from exclusion by direct-detection experiments; in fact, the allowed parameter space after constraints from perturbative unitarity, oblique parameters and $h \rightarrow \gamma \gamma$ is almost entirely unconstrained by the current direct-detection limits. As a result, both of the possible models $-n=6,8$ - remain viable after the application of all constraints. However, neither model provides a compelling explanation for dark matter, as they cannot account for more than $1 \%$ of the observed relic abundance of dark matter at masses below $1 \mathrm{TeV}$. In order to account for the full observed relic abundance, the dark matter candidate would have to be on the order of 10-30 TeV in mass. We expect that the viable parameter space of these models will become increasingly limited as results arise from next-generation dark matter experiments such as Xenon-1T (see Fig. 6.11). 
In Chapter 7, we considered the Georgi-Machacek (GM) model, which extends the SM with two scalar triplets (one real and one complex). In this model both triplets acquire a vacuum expectation value and the triplet states mix with the states of the SM doublet, resulting in an SM-like neutral scalar $h$ and nine additional BSM scalars $H, H_{3}^{0, \pm}$, and $H_{5}^{0, \pm, \pm \pm}$. An application of constraints from the requirements of treelevel perturbative unitarity and vacuum stability in the scalar potential allowed us to determine maximal ranges for the parameters $\lambda_{2,3,4,5}$ and $M_{1,2}$ (see Table 7.3).

By analysing the decoupling limit of the model, we concluded that the rate at which the BSM states will decouple — leaving a low-energy SM-like theory — depends strongly on the dimensionful parameter $M_{1}$. If $M_{1}$ is a fixed constant, most of the tree-level GM couplings (e.g. trilinear Higgs couplings, and ratios $\kappa_{f}$ and $\kappa_{V}$ ) reduce to those of the SM very quickly, at a rate inversely proportional to the mass scale of the new scalars to the fourth power, $M_{\text {new }}^{-4}$. However, if $M_{1}$ is proportional to the mass scale, these couplings decouple much more slowly, inversely proportional only to the square of the BSM mass scale, $M_{\text {new }}^{-2}$. Furthermore, the leading order decoupling term in the Higgs couplings to fermions (vector bosons) is negative (positive), indicating that these couplings will generally be suppressed with $\kappa_{f} \leq 1$ (enhanced with $\kappa_{V} \geq 1$ ) in comparison with the SM, particularly at higher mass scales $M_{\text {new }}$. These decoupling patterns differentiate the GM phenomenology from that of the 2HDM, where Higgs couplings to fermions decouple much more slowly than couplings to vector bosons proportional to $M_{\text {new }}^{-2}$ and $M_{\text {new }}^{-4}$ respectively - and tend to be enhanced $\left(\kappa_{f} \geq 1\right)$ while the vector boson couplings are suppressed $\left(\kappa_{V} \leq 1\right)$ at higher mass scales.

We have also applied constraints from oblique parameters and interaction processes involving fermions $\left(R_{b}, B_{s}^{0} \rightarrow \mu^{+} \mu^{-}, B_{s}^{0}-\bar{B}_{s}^{0}\right.$ mixing, and $\left.b \rightarrow s \gamma\right)$. By numerically scanning the parameter space under both the theoretical bounds and these 
experimental constraints, we conclude that $B R\left(\bar{B} \rightarrow X_{s} \gamma\right)$ bounds the GM triplet vev $v_{\chi} \leq 65 \mathrm{GeV}$. The couplings of the SM-like Higgs boson $h$ to fermions may be enhanced in comparison with the corresponding SM coupling by almost $50 \%$, $\kappa_{f} \leq 1.49$, while the Higgs coupling to vector bosons may be enhanced by up to $36 \%$, $\kappa_{V} \leq 1.36$. Furthermore, both types of couplings may be enhanced together by up to $18 \%, \kappa_{f}=\kappa_{V}=1.18$. This corresponds to an increase of up to $39 \%$ in all Higgs production cross sections. The GM model therefore provides a valuable benchmark as a model which may enhance all of the Higgs couplings to SM particles simultaneously. As discussed in Sec. 7.4.3 and at the beginning of Chp. 7, an understanding of this mechanism is important for the identification of Higgs models, as it may be able to suppress the effects of BSM physics in collider data [34].

In future work, dedicated collider studies of various production processes will be important in order to identify clear experimental signatures of the GM model, to obtain further constraints by recasting experimental results, and to further investigate the mechanism of enhanced Higgs couplings. To facilitate these studies, the theoretical and experimental constraints considered in Chp. 7 have been implemented in a publicly available code GMCalc [166]. However, such studies will require a clear understanding of the decay branching ratios of the GM scalars, including loop decay channels that have yet to be implemented in this code. In Sec. 7.6, we have calculated the decay rates for the GM BSM scalars to $\gamma \gamma, Z \gamma$, and $W^{+} \gamma$ and demonstrated their contribution to $H_{5}^{0}, H_{3}^{+}$, and $H_{5}^{+}$decays in a benchmark case. These channels can be important in regions of parameter space where the parent scalar is relatively light, or where the couplings controlling competing decay modes are suppressed. These decay channels will be implemented in an upcoming GMCalc update, and merit further investigation. 


\section{Appendix A}

\section{Feynman rules}

In the following we collect the relevant Feynman rules for the couplings of the new scalars in the models under consideration. In general, coupling coefficients will be defined by $C_{i j k}$ or $C_{i j k l}$, where the subscripts denote the particles involved. In all cases we will define the couplings with all particles and momenta incoming.

The Feynman rules for couplings involving only scalar particles are derived from the scalar Lagrangian $\mathcal{L} \supset-V$, where $V$ is the scalar potential that is defined for each model (see Eqs. (5.2), (6.1) and (7.10)). We will define the coupling coefficients of trilinear and quartic scalar couplings originating from this scalar potential without an overall minus sign, such that the Feynman rules are given by $-i C_{s_{1} s_{2} s_{3}}$ and $-i C_{s_{1} s_{2} s_{3} s_{4}}$ respectively. The coupling for a corresponding set of antiparticles may be obtained using $C_{s_{1}^{*} s_{2}^{*} s_{3}^{*}}=\left(C_{s_{1} s_{2} s_{3}}\right)^{*}$ and $C_{s_{1}^{*} s_{2}^{*} s_{3}^{*} s_{4}^{*}}=\left(C_{s_{1} s_{2} s_{3} s_{4}}\right)^{*}$.

The Feynman rules for the couplings of new scalars to gauge bosons come from the gauge-kinetic terms in the Lagrangian, $\mathcal{L} \supset \eta\left(\mathcal{D}_{\mu} S\right)^{\dagger}\left(\mathcal{D}^{\mu} S\right)$, where $\eta=1$ $(1 / 2)$ for a complex (real) scalar multiplet $S$ (similarly to Eq. (4.2)). The covariant 
derivative is defined by one of Eqs. (2.4-2.6), depending on the chosen basis of gauge bosons. In general, the coefficients of trilinear and quartic couplings of scalars to gauge bosons will be defined without factors of $e$, such that the Feynman rules are given by $i$ e $C_{s_{1} s_{2} V_{1}}\left(p_{1}-p_{2}\right), i e^{2} C_{s_{1} V_{1} V_{2}} g_{\mu \nu}$, and $i e^{2} C_{s_{1} s_{2} V_{1} V_{2}} g_{\mu \nu}$, where $s_{1,2}$ are scalars and $V_{1,2}$ are gauge bosons. Here we have defined $p_{1}\left(p_{2}\right)$ as the incoming momentum of the first scalar $s_{1}$ (second scalar $s_{2}$ ). Note that couplings for the corresponding set of anti-particles may be obtained using $C_{s_{1}^{*} V_{1}^{*} V_{2}^{*}}=\left(C_{s_{1} V_{1} V_{2}}\right)^{*}, C_{s_{1}^{*} s_{2}^{*} V_{1}^{*} V_{2}^{*}}=\left(C_{s_{1} s_{2} V_{1} V_{2}}\right)^{*}$, and $C_{s_{1}^{*} s_{2}^{*} V_{1}^{*}}=\left(C_{s_{2} s_{1} V_{1}}\right)^{*}=-\left(C_{s_{1} s_{2} V_{1}}\right)^{*}$.

\section{A.1 $U(1)$ Models}

Here we summarize the relevant Feynman rules involving the scalars in the models with a large multiplet invariant under a global $U(1)$ symmetry, which was studied in Chp. 5. In the following we take $Q \geq 0$ and denote the antiparticle of $\chi^{Q}$ as $\chi^{Q *}$.

The Feynman rules for the couplings of two scalars $s_{1}$ and $s_{2}$ to one or two Higgs bosons $h$ are respectively given by $-i C_{s_{1} s_{2} h}$ and $-i C_{s_{1} s_{2} h h}$, where

$$
\begin{aligned}
C_{\chi^{Q *} \chi^{Q h}} & =v\left[\lambda_{2}-\frac{1}{2} \lambda_{3}\left(Q-\frac{n-1}{2}\right)\right]=v \Lambda_{Q}, \\
C_{\chi^{Q *} \chi^{Q} h h} & =\left[\lambda_{2}-\frac{1}{2} \lambda_{3}\left(Q-\frac{n-1}{2}\right)\right]=\Lambda_{Q} .
\end{aligned}
$$

The Feynman rules for the couplings of two scalars $s_{1}$ and $s_{2}$ to one gauge boson $V_{\mu}$ are defined as $i$ e $C_{s_{1} s_{2} V}\left(p_{1}-p_{2}\right)_{\mu}$, where

$$
C_{\chi^{Q *} \chi^{Q} A}=Q
$$




$$
\begin{aligned}
C_{\chi^{Q *} \chi^{Q} Z} & =\frac{1}{s_{W} c_{W}}\left[c_{W}^{2} Q-\frac{n-1}{2}\right], \\
C_{\chi^{Q *} \chi^{Q+1} W^{-}} & =\frac{1}{s_{W}} \sqrt{\frac{(Q+1)(n-1-Q)}{2}}
\end{aligned}
$$

The Feynman rules for the couplings of two scalars $s_{1}$ and $s_{2}$ to two gauge bosons $V_{1 \mu}$ and $V_{2 \nu}$ are given by $i e^{2} C_{s_{1} s_{2} V_{1} V_{2}} g_{\mu \nu}$, where

$$
\begin{aligned}
C_{\chi^{Q *} \chi^{Q} W^{+} W^{-}} & =\frac{1}{s_{W}^{2}}\left[(2 Q+1) \frac{(n-1)}{2}-Q^{2}\right] \\
C_{\chi^{(Q+2) *} \chi^{Q} W^{+} W^{+}} & =\frac{1}{s_{W}^{2}} \sqrt{(Q+2)(n-2-Q)(Q+1)(n-1-Q)} \\
C_{\chi^{Q *} \chi^{Q} A A} & =2 Q^{2} \\
C_{\chi^{Q *} \chi^{Q} Z Z} & =\frac{2}{s_{W}^{2} c_{W}^{2}}\left[c_{W}^{2} Q-\frac{n-1}{2}\right]^{2}, \\
C_{\chi^{Q *} \chi^{Q+1} W^{-A}} & =\frac{1}{s_{W}} \sqrt{\frac{(Q+1)(n-1-Q)}{2}}(1+2 Q) \\
C_{\chi^{Q *} \chi^{Q+1} W^{-Z}} & =\frac{1}{s_{W}^{2} c_{W}} \sqrt{\frac{(Q+1)(n-1-Q)}{2}}\left[(1+2 Q) c_{W}^{2}-n+1\right] \\
C_{\chi^{Q *} \chi^{Q} Z A} & =\frac{2 Q}{s_{W} c_{W}}\left[c_{W}^{2} Q-\frac{n-1}{2}\right] .
\end{aligned}
$$

\section{A.2 $\quad Z_{2}$ Models}

Here we summarize the relevant Feynman rules for the two models with a large multiplet invariant under a $Z_{2}$ symmetry, which was studied in Chp. 6 .

\section{A.2.1 Higgs boson couplings to scalar pairs}

The Feynman rule for the coupling $s_{1} s_{2} h$ is given by $-i C_{s_{1} s_{2}}$, where 


$$
\begin{aligned}
C_{\zeta^{0, r} \zeta^{0, r} h} & =v\left(\lambda_{2}+\frac{1}{4} \lambda_{3}+\frac{n}{2}(-1)^{n / 2+1} \lambda_{4}\right), \\
C_{\zeta^{0, i} \zeta^{0, i} h} & =v\left(\lambda_{2}+\frac{1}{4} \lambda_{3}+\frac{n}{2}(-1)^{n / 2} \lambda_{4}\right), \\
C_{H_{1}^{Q} H_{1}^{-Q} h} & =v\left(\lambda_{2}+\frac{1}{4} \lambda_{3}-\frac{1}{2} \sqrt{Q^{2} \lambda_{3}^{2}+\left(n^{2}-4 Q^{2}\right) \lambda_{4}^{2}}\right), \\
C_{H_{2}^{Q} H_{2}^{-Q} h} & =v\left(\lambda_{2}+\frac{1}{4} \lambda_{3}+\frac{1}{2} \sqrt{Q^{2} \lambda_{3}^{2}+\left(n^{2}-4 Q^{2}\right) \lambda_{4}^{2}}\right), \\
C_{\zeta^{n / 2} \zeta^{-n / 2} h} & =v\left(\lambda_{2}-\frac{2 Q-1}{4} \lambda_{3}\right) .
\end{aligned}
$$

The Feynman rule for the coupling $s_{1} s_{2} h h$ is given by $-i C_{s_{1} s_{2} h h}$, where

$$
\begin{aligned}
C_{\zeta^{0, r} \zeta^{0, r} h h} & =\lambda_{2}+\frac{1}{4} \lambda_{3}+\frac{n}{2}(-1)^{n / 2+1} \lambda_{4}, \\
C_{\zeta^{0, i} \zeta^{0, i} h h} & =\lambda_{2}+\frac{1}{4} \lambda_{3}+\frac{n}{2}(-1)^{n / 2} \lambda_{4}, \\
C_{H_{1}^{Q} H_{1}^{-Q} h h} & =\lambda_{2}+\frac{1}{4} \lambda_{3}-\frac{1}{2} \sqrt{Q^{2} \lambda_{3}^{2}+\left(n^{2}-4 Q^{2}\right) \lambda_{4}^{2}}, \\
C_{H_{2}^{Q} H_{2}^{-Q} h h} & =\lambda_{2}+\frac{1}{4} \lambda_{3}+\frac{1}{2} \sqrt{Q^{2} \lambda_{3}^{2}+\left(n^{2}-4 Q^{2}\right) \lambda_{4}^{2}}, \\
C_{\zeta^{n / 2} \zeta^{-n / 2} h h} & =\lambda_{2}-\frac{2 Q-1}{4} \lambda_{3} .
\end{aligned}
$$

\section{A.2.2 Gauge boson couplings to scalar pairs}

\section{Couplings to one or two photons}

The Feynman rule for the coupling $s s^{*} A_{\mu}$ is $i e C_{s s^{*} A}\left(p_{1}-p_{2}\right)_{\mu}$, where $C_{s s^{*} A}=Q_{s}$ and $Q_{s}$ is the electric charge of scalar $s$ in units of $e$. Similarly, the Feynman rule for the

coupling $s s^{*} A_{\mu} A_{\nu}$ is $i e^{2} C_{s s^{*} A A} g_{\mu \nu}$, where $C_{s s^{*} A A}=2 Q_{s}^{2}$. There are no off-diagonal couplings, in accordance with the conservation of the electromagnetic current. 


\section{Couplings to one $Z$ boson}

The Feynman rule for the coupling $s_{1} s_{2} Z_{\mu}$ is given by $i e C_{s_{1} s_{2} Z}\left(p_{1}-p_{2}\right)_{\mu}$, where

$$
\begin{aligned}
C_{\zeta^{0, r} \zeta^{0, i} Z} & =\frac{i}{2 s_{W} c_{W}}, \\
C_{H_{1}^{Q} H_{1}^{-Q} Z} & =\frac{1}{s_{W} c_{W}}\left[\left(Q-\frac{1}{2}\right) \cos ^{2} \alpha_{Q}+\left(Q+\frac{1}{2}\right) \sin ^{2} \alpha_{Q}-Q s_{W}^{2}\right], \\
C_{H_{2}^{Q} H_{2}^{-Q} Z} & =\frac{1}{s_{W} c_{W}}\left[\left(Q-\frac{1}{2}\right) \sin ^{2} \alpha_{Q}+\left(Q+\frac{1}{2}\right) \cos ^{2} \alpha_{Q}-Q s_{W}^{2}\right], \\
C_{H_{1}^{Q} H_{2}^{-Q} Z} & =C_{H_{2}^{Q} H_{1}^{-Q}}=\frac{1}{s_{W} c_{W}} \sin \alpha_{Q} \cos \alpha_{Q}, \\
C_{\zeta^{n / 2} \zeta^{-n / 2} Z} & =\frac{1}{s_{W} c_{W}}\left[\frac{n-1}{2}-\frac{n}{2} s_{W}^{2}\right] .
\end{aligned}
$$

Note that the diagonal couplings $C_{\zeta^{0, r} \zeta^{0, r} Z}=C_{\zeta^{0, i} \zeta^{0, i} Z}=0$ due to parity conservation.

\section{Couplings to $Z Z$}

The Feynman rule for the coupling $s_{1} s_{2} Z_{\mu} Z_{\nu}$ is given by $i e^{2} C_{s_{1} s_{2} Z Z} g_{\mu \nu}$, where

$$
\begin{aligned}
C_{\zeta^{0, r} \zeta^{0, r} Z Z} & =C_{\zeta^{0, i} \zeta^{0, i} Z Z}=\frac{1}{2 s_{W}^{2} c_{W}^{2}}, \\
C_{H_{1}^{Q} H_{1}^{-Q} Z Z} & =\frac{2}{s_{W}^{2} c_{W}^{2}}\left[\left(Q c_{W}^{2}-\frac{1}{2}\right)^{2} \cos ^{2} \alpha_{Q}+\left(Q c_{W}^{2}+\frac{1}{2}\right)^{2} \sin ^{2} \alpha_{Q}\right], \\
C_{H_{1}^{Q} H_{2}^{-Q} Z Z} & =C_{H_{2}^{Q} H_{1}^{-Q}}=\frac{4 Q}{s_{W}^{2} c_{W}^{2}}\left(1-s_{W}^{2}\right) \sin \alpha_{Q} \cos \alpha_{Q}, \\
C_{H_{2}^{Q} H_{2}^{-Q} Z Z} & =\frac{2}{s_{W}^{2} c_{W}^{2}}\left[\left(Q c_{W}^{2}-\frac{1}{2}\right)^{2} \sin ^{2} \alpha_{Q}+\left(Q c_{W}^{2}+\frac{1}{2}\right)^{2} \cos ^{2} \alpha_{Q}\right], \\
C_{\zeta^{n / 2} \zeta^{-n / 2} Z Z} & =\frac{2}{s_{W}^{2} c_{W}^{2}}\left[\frac{n-1}{2}-\frac{n}{2} s_{W}^{2}\right]^{2} .
\end{aligned}
$$

Note that the off-diagonal coupling $\zeta^{0, r} \zeta^{0, i} Z Z$ is zero.

\section{Couplings to one $W$ boson}

The Feynman rule for the coupling $s_{1} s_{2} W_{\mu}^{ \pm}$is given by $i e C_{s_{1} s_{2} W^{ \pm}}\left(p_{1}-p_{2}\right)_{\mu}$, where 


$$
\begin{aligned}
C_{\zeta^{0, r} H_{1}^{-} W^{+}} & =\frac{1}{2 s_{W}}\left[\frac{n}{2} \cos \alpha_{1}-T_{-1}^{+} \sin \alpha_{1}\right] \\
C_{\zeta^{0, r} H_{2}^{-} W^{+}} & =\frac{1}{2 s_{W}}\left[-\frac{n}{2} \sin \alpha_{1}-T_{-1}^{+} \cos \alpha_{1}\right] \\
C_{\zeta^{0, i} H_{1}^{-} W^{+}} & =\frac{i}{2 s_{W}}\left[\frac{n}{2} \cos \alpha_{1}+T_{-1}^{+} \sin \alpha_{1}\right] \\
C_{\zeta^{0, i} H_{2}^{-} W^{+}} & =\frac{i}{2 s_{W}}\left[-\frac{n}{2} \sin \alpha_{1}+T_{-1}^{+} \cos \alpha_{1}\right] \\
C_{H_{1}^{Q} H_{1}^{-(Q+1)} W^{+}} & =\frac{1}{\sqrt{2} s_{W}}\left[T_{Q}^{+} \cos \alpha_{Q} \cos \alpha_{Q+1}-T_{-Q-1}^{+} \sin \alpha_{Q} \sin \alpha_{Q+1}\right] \\
C_{H_{1}^{Q} H_{2}^{-(Q+1)} W^{+}} & =\frac{1}{\sqrt{2} s_{W}}\left[-T_{Q}^{+} \cos \alpha_{Q} \sin \alpha_{Q+1}-T_{-Q-1}^{+} \sin \alpha_{Q} \cos \alpha_{Q+1}\right], \\
C_{H_{2}^{Q} H_{1}^{-(Q+1)} W^{+}} & =\frac{1}{\sqrt{2} s_{W}}\left[-T_{Q}^{+} \sin \alpha_{Q} \cos \alpha_{Q+1}-T_{-Q-1}^{+} \cos \alpha_{Q} \sin \alpha_{Q+1}\right], \\
C_{H_{2}^{Q} H_{2}^{-(Q+1)} W^{+}} & =\frac{1}{\sqrt{2} s_{W}}\left[T_{Q}^{+} \sin \alpha_{Q} \sin \alpha_{Q+1}-T_{-Q-1}^{+} \cos \alpha_{Q} \cos \alpha_{Q+1}\right] \\
C_{H_{1}^{n / 2-1} \zeta^{-n / 2} W^{+}} & =\frac{1}{\sqrt{2} s_{W}} T_{n / 2-1}^{+} \cos \alpha_{n / 2-1} \\
C_{H_{2}^{n / 2-1} \zeta^{-n / 2} W^{+}} & =\frac{1}{\sqrt{2} s_{W}} T_{n / 2-1}^{+} \sin \alpha_{n / 2-1} .
\end{aligned}
$$

For compactness, we have defined the following coefficients for a given value of $n$ :

$$
\begin{aligned}
T_{Q}^{+} & =\frac{1}{2} \sqrt{n^{2}-4 Q^{2}}, \\
T_{Q}^{-} & =\frac{1}{2} \sqrt{n^{2}-4(Q-1)^{2}} .
\end{aligned}
$$

Note that all the couplings $C_{s_{1} s_{2} W^{+}}$are real except for those that involve one $\zeta^{0, i}$, which are imaginary.

\section{Couplings to $W^{+} W^{-}$}

The Feynman rule for the coupling $s_{1} s_{2} W_{\mu}^{+} W_{\nu}^{-}$is given by $i e^{2} C_{s_{1} s_{2} W^{+} W^{-}} g_{\mu \nu}$, where

$$
\begin{aligned}
C_{\zeta^{0, r} \zeta^{0, r} W^{+} W^{-}} & =C_{\zeta^{0, i} \zeta^{0, i} W^{+} W^{-}}=\frac{1}{2 s_{W}^{2}} T_{0}^{+-}, \\
C_{H_{1}^{Q} H_{1}^{-Q} W^{+} W^{-}} & =\frac{1}{2 s_{W}^{2}}\left[T_{Q}^{+-} \cos ^{2} \alpha_{Q}+T_{Q}^{-+} \sin ^{2} \alpha_{Q}\right],
\end{aligned}
$$




$$
\begin{aligned}
C_{H_{2}^{Q} H_{2}^{-Q} W^{+} W^{-}} & =\frac{1}{2 s_{W}^{2}}\left[T_{Q}^{+-} \sin ^{2} \alpha_{2}+T_{Q}^{-+} \cos ^{2} \alpha_{2}\right], \\
C_{H_{1}^{Q} H_{2}^{-Q} W^{+} W^{-}} & =C_{H_{2}^{Q} H_{1}^{-Q} W^{+} W^{-}}=\frac{2 Q}{s_{W}^{2}} \sin \alpha_{Q} \cos \alpha_{Q}, \\
C_{\zeta^{n / 2} \zeta^{-n / 2} W^{+} W^{-}} & =\frac{1}{2 s_{W}^{2}} T_{n / 2}^{+-} .
\end{aligned}
$$

Again, for compactness we have defined

$$
\begin{aligned}
& T_{Q}^{+-}=T_{Q}^{+} T_{Q+1}^{-}+T_{Q}^{-} T_{Q-1}^{+}=\frac{n^{2}-2}{2}-2 Q(Q-1), \\
& T_{Q}^{-+}=T_{Q}^{-} T_{Q-1}^{+}+T_{Q}^{+} T_{Q+1}^{-}=\frac{n^{2}-2}{2}-2 Q(Q+1) .
\end{aligned}
$$

for a given value of $n$. Note that the off-diagonal coupling $\zeta^{0, r} \zeta^{0, i} W_{\mu}^{+} W_{\nu}^{-}$is zero.

Further couplings to $Z \gamma, W^{ \pm} \gamma, W^{ \pm} W^{ \pm}$, and $W^{ \pm} Z$ can be found in the appendix of Ref. [3].

\section{A.3 Georgi-Machacek}

Here we summarize the relevant Feynman rules for the Georgi-Machacek model, which was studied in Chp. 7. Additional rules (involving Goldstone bosons and couplings of form $s_{1} s_{2} V_{1} V_{2}$ ) can be found in Ref. [4].

\section{A.3.1 Triple-scalar couplings}

\section{Couplings involving $h$}

The Feynman rules for three-scalar couplings involving $h$ are given by $-i C_{s_{1} s_{2} h}$, with all particles incoming and the couplings defined as follows: 


$$
\begin{aligned}
& C_{h h h}= 24 \lambda_{1} c_{\alpha}^{3} v_{\phi}-6 s_{\alpha} c_{\alpha}\left(\sqrt{3} c_{\alpha} v_{\chi}-s_{\alpha} v_{\phi}\right)\left(2 \lambda_{2}-\lambda_{5}\right)-8 \sqrt{3} s_{\alpha}^{3} v_{\chi}\left(\lambda_{3}+3 \lambda_{4}\right) \\
&+\frac{3 \sqrt{3}}{2} M_{1} c_{\alpha}^{2} s_{\alpha}-4 \sqrt{3} M_{2} s_{\alpha}^{3} \\
& C_{H h h}= 24 \lambda_{1} c_{\alpha}^{2} s_{\alpha} v_{\phi}+2\left[\sqrt{3} c_{\alpha} v_{\chi}\left(3 c_{\alpha}^{2}-2\right)+s_{\alpha} v_{\phi}\left(1-3 c_{\alpha}^{2}\right)\right]\left(2 \lambda_{2}-\lambda_{5}\right) \\
&+8 \sqrt{3} c_{\alpha} s_{\alpha}^{2} v_{\chi}\left(\lambda_{3}+3 \lambda_{4}\right)-\frac{\sqrt{3}}{2} M_{1} c_{\alpha}\left(3 c_{\alpha}^{2}-2\right)-4 \sqrt{3} M_{2} c_{\alpha} s_{\alpha}^{2} \\
& C_{H H h}= 24 \lambda_{1} c_{\alpha} s_{\alpha}^{2} v_{\phi}+2\left[\sqrt{3} s_{\alpha} v_{\chi}\left(3 c_{\alpha}^{2}-1\right)+c_{\alpha} v_{\phi}\left(3 c_{\alpha}^{2}-2\right)\right]\left(2 \lambda_{2}-\lambda_{5}\right) \\
&-8 \sqrt{3} c_{\alpha}^{2} s_{\alpha} v_{\chi}\left(\lambda_{3}+3 \lambda_{4}\right)-\frac{\sqrt{3}}{2} M_{1} s_{\alpha}\left(3 c_{\alpha}^{2}-1\right)+4 \sqrt{3} M_{2} c_{\alpha}^{2} s_{\alpha} \\
& C_{H_{3}^{0} H_{3}^{0} h}= C_{H_{3}^{+}} H_{3}^{+*} h^{2}=64 \lambda_{1} c_{\alpha} \frac{v_{\chi}^{2} v_{\phi}}{v^{2}}-\frac{8}{\sqrt{3}} \frac{v_{\phi}^{2} v_{\chi}}{v^{2}} s_{\alpha}\left(\lambda_{3}+3 \lambda_{4}\right)+\frac{2 \sqrt{3} M_{2} v_{\phi}^{2}}{v^{2}} s_{\alpha} \\
&-\frac{16}{\sqrt{3}} \frac{v_{\chi}^{3}}{v^{2}} s_{\alpha}\left(6 \lambda_{2}+\lambda_{5}\right)-\frac{4}{\sqrt{3}} \frac{v_{\chi} M_{1}}{v^{2}}\left(s_{\alpha} v_{\chi}-\sqrt{3} c_{\alpha} v_{\phi}\right) \\
&-\frac{8}{\sqrt{3}} \lambda_{5} \frac{v_{\chi} v_{\phi}}{v^{2}}\left(s_{\alpha} v_{\phi}-\sqrt{3} c_{\alpha} v_{\chi}\right)-c_{\alpha} \frac{v_{\phi}^{3}}{v^{2}}\left(\lambda_{5}-4 \lambda_{2}\right) \\
&= C_{H_{5}^{+}} H_{5}^{+*} h=C_{H_{5}^{++}} H_{5}^{++*} h \\
&=-8 \sqrt{3}\left(\lambda_{3}+\lambda_{4}\right) s_{\alpha} v_{\chi}+\left(4 \lambda_{2}+\lambda_{5}\right) c_{\alpha} v_{\phi}-2 \sqrt{3} M_{2} s_{\alpha} \\
& C_{H_{5}^{0} H_{5}^{0} h}
\end{aligned}
$$

Here we use $s_{\alpha} \equiv \sin \alpha$ and $c_{\alpha} \equiv \cos \alpha$. Accounting for the mixing $\phi^{0, r}, H_{1}^{0 \prime} \rightarrow h, H$ and the difference in notation, the couplings agree with those of Ref. [137] in the $M_{1}=M_{2}=0$ limit (after correcting a small typo in the $H_{1}^{0} H_{3}^{+} H_{3}^{-}$coupling).

\section{Couplings involving $H$}

The rules for three-scalar couplings involving $H$ are given by $-i C_{s_{1} s_{2} H}$, where

$$
\begin{aligned}
C_{H H H}= & 24 \lambda_{1} s_{\alpha}^{3} v_{\phi}+6 s_{\alpha} c_{\alpha}\left(\sqrt{3} s_{\alpha} v_{\chi}+c_{\alpha} v_{\phi}\right)\left(2 \lambda_{2}-\lambda_{5}\right)+8 \sqrt{3} c_{\alpha}^{3} v_{\chi}\left(\lambda_{3}+3 \lambda_{4}\right) \\
& -\frac{3 \sqrt{3}}{2} M_{1} s_{\alpha}^{2} c_{\alpha}-4 \sqrt{3} M_{2} c_{\alpha}^{3}
\end{aligned}
$$




$$
\begin{aligned}
C_{H_{3}^{0} H_{3}^{0} H}= & C_{H_{3}^{+} H_{3}^{+*} H} \\
= & 64 \lambda_{1} s_{\alpha} \frac{v_{\chi}^{2} v_{\phi}}{v^{2}}+\frac{8}{\sqrt{3}} \frac{v_{\phi}^{2} v_{\chi}}{v^{2}} c_{\alpha}\left(\lambda_{3}+3 \lambda_{4}\right)+\frac{4}{\sqrt{3}} \frac{v_{\chi} M_{1}}{v^{2}}\left(c_{\alpha} v_{\chi}+\sqrt{3} s_{\alpha} v_{\phi}\right) \\
& +\frac{16}{\sqrt{3}} \frac{v_{\chi}^{3}}{v^{2}} c_{\alpha}\left(6 \lambda_{2}+\lambda_{5}\right)+s_{\alpha} \frac{v_{\phi}^{3}}{v^{2}}\left(4 \lambda_{2}-\lambda_{5}\right)-\frac{2 \sqrt{3} M_{2} v_{\phi}^{2}}{v^{2}} c_{\alpha} \\
& +\frac{8}{\sqrt{3}} \lambda_{5} \frac{v_{\chi} v_{\phi}}{v^{2}}\left(c_{\alpha} v_{\phi}+\sqrt{3} s_{\alpha} v_{\chi}\right) \\
C_{H_{5}^{0} H_{5}^{0} H}= & C_{H_{5}^{+} H_{5}^{+*} H}=C_{H_{5}^{++}} H_{5}^{++*} H \\
= & 8 \sqrt{3}\left(\lambda_{3}+\lambda_{4}\right) c_{\alpha} v_{\chi}+\left(4 \lambda_{2}+\lambda_{5}\right) s_{\alpha} v_{\phi}+2 \sqrt{3} M_{2} c_{\alpha}
\end{aligned}
$$

\section{Couplings involving $H_{3}$ and $H_{5}$}

The Feynman rules for three-scalar couplings involving scalars from the custodial

triplet and fiveplet are given by $-i C_{s_{1} s_{2} s_{3}}$, with all particles incoming and the couplings defined as follows:

$$
\begin{aligned}
C_{H_{3}^{0} H_{3}^{0} H_{5}^{0}} & =-\frac{2 \sqrt{2}}{\sqrt{3} v^{2}}\left(-8 \lambda_{5} v_{\chi}^{3}+4 M_{1} v_{\chi}^{2}+\left(-4 \lambda_{5}+2 \lambda_{3}\right) v_{\phi}^{2} v_{\chi}+3 M_{2} v_{\phi}^{2}\right) \\
C_{H_{3}^{+} H_{3}^{+*} H_{5}^{0}} & =\frac{\sqrt{2}}{\sqrt{3} v^{2}}\left(-8 \lambda_{5} v_{\chi}^{3}+4 M_{1} v_{\chi}^{2}+\left(-4 \lambda_{5}+2 \lambda_{3}\right) v_{\phi}^{2} v_{\chi}+3 M_{2} v_{\phi}^{2}\right), \\
C_{H_{3}^{0} H_{3}^{+} H_{5}^{+*}} & =-i \frac{\sqrt{2}}{v^{2}}\left(-8 \lambda_{5} v_{\chi}^{3}+4 M_{1} v_{\chi}^{2}+\left(-4 \lambda_{5}+2 \lambda_{3}\right) v_{\phi}^{2} v_{\chi}+3 M_{2} v_{\phi}^{2}\right), \\
C_{H_{3}^{+} H_{3}^{+} H_{5}^{++*}} & =-\frac{2}{v^{2}}\left(-8 \lambda_{5} v_{\chi}^{3}+4 M_{1} v_{\chi}^{2}+\left(-4 \lambda_{5}+2 \lambda_{3}\right) v_{\phi}^{2} v_{\chi}+3 M_{2} v_{\phi}^{2}\right), \\
C_{H_{5}^{0} H_{5}^{0} H_{5}^{0}} & =2 \sqrt{6}\left(2 \lambda_{3} v_{\chi}-M_{2}\right) \\
C_{H_{5}^{+} H_{5}^{+*} H_{5}^{0}} & =\sqrt{6}\left(2 \lambda_{3} v_{\chi}-M_{2}\right) \\
C_{H_{5}^{+} H_{5}^{+} H_{5}^{++*}} & =-6\left(2 \lambda_{3} v_{\chi}-M_{2}\right) \\
C_{H_{5}^{++} H_{5}^{++*} H_{5}^{0}} & =-2 \sqrt{6}\left(2 \lambda_{3} v_{\chi}-M_{2}\right) .
\end{aligned}
$$




\section{A.3.2 Scalar couplings to gauge bosons}

We note that the couplings listed in this subsection are consistent with those in Ref. [92] after taking into account the different sign convention in the covariant derivative and the different sign in the definition of the neutral custodial triplet state $H_{3}^{0}$, equal to $-H_{3}^{0}$ in our notation. Recall that $\phi$ and $\chi$ are complex but $\xi$ is real.

\section{Couplings of one scalar to two gauge bosons}

The Feynman rules for the vertices involving a scalar and two gauge bosons are defined as $i e^{2} C_{s V_{1} V_{2}} g^{\mu \nu}$. The couplings are given by

$$
\begin{aligned}
C_{h W^{+} W^{+*}} & =c_{W}^{2} C_{h Z Z}=-\frac{1}{6 s_{W}^{2}}\left(8 \sqrt{3} s_{\alpha} v_{\chi}-3 c_{\alpha} v_{\phi}\right), \\
C_{H W^{+} W^{+*}} & =c_{W}^{2} g_{H Z Z}=\frac{1}{6 s_{W}^{2}}\left(8 \sqrt{3} c_{\alpha} v_{\chi}+3 s_{\alpha} v_{\phi}\right), \\
C_{H_{5}^{0} W^{+} W^{+*}} & =\sqrt{\frac{2}{3}} \frac{1}{s_{W}^{2}} v_{\chi}, \\
C_{H_{5}^{0} Z Z} & =-\sqrt{\frac{8}{3}} \frac{1}{s_{W}^{2} c_{W}^{2}} v_{\chi}, \\
C_{H_{5}^{+} W^{+*} Z} & =-\frac{\sqrt{2} v_{\chi}}{c_{W} s_{W}^{2}} \\
C_{H_{5}^{++} W^{+*} W^{+*}} & =\frac{2 v_{\chi}}{s_{W}^{2}} .
\end{aligned}
$$

\section{Couplings of two scalars to one photon}

The Feynman rules for the vertices involving two scalars and a single photon $\gamma_{\mu}$ are defined as $i e C_{s_{1} s_{2} A}\left(p_{1}-p_{2}\right)_{\mu}$. The couplings are given by $C_{\gamma s s^{*}}=Q_{s}$, where $s_{1}=s$ and $s_{2}=s_{1}^{*}=s^{*}$ and $Q_{s}$ being the electric charge of the scalar $s$ in units of $e$. All off-diagonal couplings and neutral scalar couplings to the photon are zero. 


\section{Couplings of two scalars to one $Z$ boson}

The Feynman rules for the vertices involving two scalars and a single $Z$ boson are

defined as $i e C_{Z s_{1} s_{2}}\left(p_{1}-p_{2}\right)_{\mu}$, where again $p_{1}\left(p_{2}\right)$ is the incoming momentum of incoming scalar $s_{1}\left(s_{2}\right)$. The couplings are given by

$$
\begin{aligned}
C_{Z h H_{3}^{0}} & =-i \sqrt{\frac{2}{3}} \frac{1}{s_{W} c_{W}}\left(\sqrt{3} \frac{v_{\chi}}{v} c_{\alpha}+s_{\alpha} \frac{v_{\phi}}{v}\right), \\
C_{Z H H_{3}^{0}} & =i \sqrt{\frac{2}{3}} \frac{1}{s_{W} c_{W}}\left(c_{\alpha} \frac{v_{\phi}}{v}-\sqrt{3} \frac{v_{\chi}}{v} s_{\alpha}\right), \\
C_{Z H_{3}^{0} H_{5}^{0}} & =i \sqrt{\frac{1}{3}} \frac{1}{s_{W} c_{W}} \frac{v_{\phi}}{v}, \\
C_{Z H_{3}^{+} H_{3}^{+*}} & =C_{Z H_{5}^{+} H_{5}^{+*}}=C_{Z G^{+} G^{+*}}=\frac{e}{2 s_{W} c_{W}}\left(1-2 s_{W}^{2}\right), \\
C_{Z H_{5}^{++} H_{5}^{++*}} & =\frac{1}{s_{W} c_{W}}\left(1-2 s_{W}^{2}\right), \\
C_{Z H_{3}^{+} H_{5}^{+*}} & =-\frac{1}{2 s_{W} c_{W}} \frac{v_{\phi}}{v} .
\end{aligned}
$$

Note that all the diagonal couplings of $Z$ to charged scalars have the form

$$
C_{Z s s^{*}}=\frac{Q_{s}}{2 s_{W} c_{W}}\left(1-2 s_{W}^{2}\right)
$$

where $Q_{s}$ is the electric charge of $s$. This simple form is a consequence of custodial $\mathrm{SU}(2)$ symmetry.

\section{Couplings of two scalars to one $W$ boson}

The Feynman rules for the vertices involving two scalars and a single $W^{+}$boson are defined as $i C_{W^{+} s_{1} s_{2}}\left(p_{1}-p_{2}\right)_{\mu}$, where again $p_{1}\left(p_{2}\right)$ is the incoming momentum of incoming scalar $s_{1}\left(s_{2}\right)$. The couplings are given by

$$
C_{W^{+} h H_{3}^{+*}}=-\sqrt{\frac{2}{3}} \frac{1}{s_{W}}\left(\sqrt{3} c_{\alpha} \frac{v_{\chi}}{v}+s_{\alpha} \frac{v_{\phi}}{v}\right),
$$




$$
\begin{aligned}
C_{W^{+} H H_{3}^{+*}} & =-\sqrt{\frac{2}{3}} \frac{1}{s_{W}}\left(\sqrt{3} s_{\alpha} \frac{v_{\chi}}{v}-c_{\alpha} \frac{v_{\phi}}{v}\right), \\
C_{W^{+} H_{3}^{0} H_{3}^{+*}} & =-\frac{i}{2} \frac{1}{s_{W}} \\
C_{W^{+} H_{3}^{+*} H_{5}^{0}} & =-\frac{1}{2 \sqrt{3}} \frac{1}{s_{W}} \frac{v_{\phi}}{v}, \\
C_{W^{+} H_{5}^{+*} H_{5}^{0}} & =\frac{\sqrt{3}}{2} \frac{1}{s_{W}} \\
C_{W^{+} H_{3}^{0} H_{5}^{+*}} & =\frac{i}{2} \frac{1}{s_{W}} \frac{v_{\phi}}{v}, \\
C_{W^{+} H_{3}^{+} H_{5}^{++*}} & =\frac{1}{\sqrt{2}} \frac{1}{s_{W}} \frac{v_{\phi}}{v}, \\
C_{W^{+} H_{5}^{+} H_{5}^{++*}} & =\frac{1}{\sqrt{2}} \frac{1}{s_{W}} .
\end{aligned}
$$

\section{A.3.3 Scalar couplings to fermions}

The Feynman rules for the vertices involving a CP-even neutral scalar $s=h, H$ and two charged fermions $\bar{f} f$ are given by $i C_{s \bar{f} f}$, where

$$
C_{h \bar{f} f}=-\frac{m_{f}}{v} \frac{\cos \alpha}{\cos \theta_{H}}, \quad C_{H \bar{f} f}=-\frac{m_{f}}{v} \frac{\sin \alpha}{\cos \theta_{H}}
$$

Similarly, the rules for vertices involving a CP-odd neutral scalar $H_{3}^{0}$ and two quarks $q=u, d$ is given by $C_{H_{3}^{0} \bar{q} q} \gamma_{5},{ }^{1}$ where

$$
C_{H_{3}^{0} \bar{u} u}=\frac{m_{u}}{v} \tan \theta_{H}, \quad C_{H_{3}^{0} \bar{d} d}=-\frac{m_{d}}{v} \tan \theta_{H}
$$

Here $u$ stands for any up-type quark, and $d$ stands for any down-type quark or charged lepton.

The Feynman rules for the vertices involving a charged scalar $H_{3}^{+}$and two

\footnotetext{
${ }^{1}$ Note that in the $i\left(C^{S}+C^{P} \gamma_{5}\right)$ convention the CP-even couplings $C_{s \bar{f} f}=C^{S}$, while the CP-odd $C_{H_{3}^{0} \bar{q} q}=i C^{P}$.
} 
fermions $\bar{f}_{1} f_{2}$ are given by $i\left(C_{H_{3}^{+} \bar{f}_{1} f_{2}}^{S}+C_{H_{3}^{+} \bar{f}_{1} f_{2}}^{P} \gamma_{5}\right)$, where

$$
\begin{aligned}
C_{H_{3}^{+} \bar{u} d}^{S} & =-\frac{\sqrt{2}}{2 v} V_{u d} \tan \theta_{H}\left(m_{u}-m_{d}\right) \\
C_{H_{3}^{+} \bar{u} d}^{P} & =-\frac{\sqrt{2}}{2 v} V_{u d} \tan \theta_{H}\left(-m_{u}-m_{d}\right) \\
C_{H_{3}^{+*} \bar{d} u}^{S} & =-\frac{\sqrt{2}}{2 v} V_{u d}^{*} \tan \theta_{H}\left(m_{u}-m_{d}\right) \\
C_{H^{+*} \bar{d} u}^{P} & =-\frac{\sqrt{2}}{2 v} V_{u d}^{*} \tan \theta_{H}\left(m_{u}+m_{d}\right) \\
C_{H_{3}^{+} \bar{\nu} \ell}^{S} & =\frac{\sqrt{2}}{2 v} \tan \theta_{H} m_{\ell} \\
C_{H_{3}^{+} \bar{\nu} \ell}^{P} & =\frac{\sqrt{2}}{2 v} \tan \theta_{H} m_{\ell} \\
C_{H_{3}^{+*} \bar{\ell} \nu}^{S} & =\frac{\sqrt{2}}{2 v} \tan \theta_{H} m_{\ell} \\
C_{H_{3}^{+*} \bar{\ell} \nu}^{P} & =-\frac{\sqrt{2}}{2 v} \tan \theta_{H} m_{\ell} .
\end{aligned}
$$

Here $V_{u d}$ is the appropriate element of the CKM matrix.

The custodial fiveplet states do not couple to fermions. 


\section{Appendix B}

\section{Scalar decays in the GM model}

\section{B.1 Direct decays to $W$ and $Z$ vector bosons}

The tree-level decays of a scalar $H_{i}$ to two vector bosons $V_{1} V_{2}$ - where $V_{1} V_{2}=$ $Z Z, W W, W Z$ - are described by [166]

$$
\begin{aligned}
\Gamma\left(H_{i} \rightarrow V_{1} V_{2}\right)= & S_{V} \frac{\left|e C_{H_{i} V_{1} V_{2}}\right|^{2} m_{H_{i}}^{3}}{64 \pi m_{V_{1}}^{2} m_{V_{1}}^{2}} \lambda^{\frac{1}{2}}\left(\frac{m_{V_{1}}^{2}}{m_{H_{i}}^{2}}, \frac{m_{V_{2}}^{2}}{m_{H_{i}}^{2}}\right) \\
& \times\left[1-2 \frac{m_{V_{1}}^{2}+m_{V_{2}}^{2}}{m_{H_{i}}^{2}}+\frac{10 m_{V_{1}}^{2} m_{V_{2}}^{2}+m_{V_{1}}^{4}+m_{V_{2}}^{4}}{m_{H_{i}}^{4}}\right]
\end{aligned}
$$

where $S_{V}=1(1 / 2)$ for $W^{+} W^{-}$and $W^{+} Z(Z Z)$, and the kinematic factor

$$
\lambda(x, y)=(1-x-y)^{2}-4 x y .
$$

\section{B.2 Decays to off-shell vector bosons}

In decays to two vector bosons, in order to account for decays where one or both of the outgoing gauge bosons are off-shell we make use of $[176,177]$ 


$$
\begin{aligned}
\Gamma\left(H_{i} \rightarrow V_{1}^{*} V_{2}^{*}\right)= & \frac{1}{\pi^{2}} \int d Q_{1}^{2} \frac{\Gamma_{V_{1}}}{m_{V_{1}}} \frac{Q_{1}^{2}}{\left(Q_{1}^{2}-m_{V_{1}}^{2}\right)^{2}+m_{V_{1}}^{2} \Gamma_{V_{1}}^{2}} \\
& \times \int d Q_{2}^{2} \frac{\Gamma_{V_{2}}}{m_{V_{2}}} \frac{Q_{2}^{2}}{\left(Q_{2}^{2}-m_{V_{2}}^{2}\right)^{2}+m_{V_{2}}^{2} \Gamma_{V_{2}}^{2}} \Gamma^{H_{i} V_{1} V_{2}}\left(Q_{1}^{2}, Q_{2}^{2}\right),
\end{aligned}
$$

where $\Gamma_{V_{i}}$ is the total width of $V_{i}$ and $\Gamma^{H_{i} V_{1} V_{2}}\left(Q_{1}^{2}, Q_{2}^{2}\right)$ is the on-shell decay width for $H_{i} \rightarrow V_{1} V_{2}$ where the invariant masses for the gauge bosons $V_{1}$ and $V_{2}$ have been replaced by $Q_{1}$ and $Q_{2}$. Similarly, if only one gauge boson is off-shell, then

$$
\Gamma\left(H_{i} \rightarrow V_{1}^{*} V_{2}\right)=\frac{1}{\pi} \int d Q_{1}^{2} \frac{\Gamma_{V_{1}}}{m_{V_{1}}} \frac{Q_{1}^{2} \Gamma^{H_{i} V_{1} V_{2}}\left(Q_{1}^{2}, Q_{2}^{2}\right)}{\left(Q_{1}^{2}-m_{V_{1}}^{2}\right)^{2}+m_{V_{1}}^{2} \Gamma_{V_{1}}^{2}} .
$$

In the case of loop decays to $Z \gamma$ and $W^{+} \gamma$, when the $Z$ or $W$ boson is offshell additional non-resonant diagrams will contribute at the same order. As we have not considered these contributions, the off-shell results for the loop decays should be considered an approximation.

\section{B.3 Decays to gluons}

The $H_{3}^{0}$ CP-odd scalar may also decay to two gluons through a fermion loop, as described by

$$
\tilde{A}_{t o t}^{H_{3}^{0} g g}=\frac{\alpha_{s} g}{4 \pi m_{W}}\left(-2 \beta_{f}^{H_{3}^{0}} \sum_{f} \tau_{f} f\left(\tau_{f}\right)\right) .
$$

Note that the argument under the sum is the same as that of the $\gamma \gamma$ decay given by Eqs. (7.128) and (7.129), except without the factor of $N_{c, f} Q_{f}^{2}$ and with the exchange of the electroweak coupling constant for the strong coupling constant $\alpha_{\mathrm{em}} \rightarrow \alpha_{s}$. The value of $\alpha_{s}$ may be found for different scales $\mu$ using

$$
\alpha_{s}(\mu)=\frac{\alpha_{s}\left(m_{Z}\right)}{v_{s}(\mu)}\left(1-\frac{\beta_{1}}{\beta_{0}} \frac{\alpha_{s}\left(m_{Z}\right)}{4 \pi} \frac{\ln v_{s}(\mu)}{v_{s}(\mu)}\right),
$$


where $\alpha_{s}\left(m_{Z}\right)=0.11856[43]$ and

$$
v_{s}(\mu)=1+\beta_{0} \frac{\alpha_{s}\left(m_{Z}\right)}{2 \pi} \ln \frac{\mu}{m_{Z}}, \quad \beta_{0}=11-\frac{2}{3} n_{f} \quad, \quad \beta_{1}=102-\frac{38}{3} n_{f} .
$$

Here $n_{f}$ is the number of effective active quark flavours, which we take to be five. We evaluate $\alpha_{s}$ at the scale of the parent particle's mass.

Keeping only the top quark contribution, we note that $h \rightarrow g g$ has the same functional structure as $h \rightarrow \gamma \gamma$. Specifically, $B R\left(H_{3}^{0} \rightarrow g g\right) / B R\left(H_{3}^{0} \rightarrow \gamma \gamma\right)=$ $9 \alpha_{s}^{2} / 16 \alpha_{\mathrm{em}}^{2}>1$. Therefore the decay to gluons will always dominate the diphoton decay for the CP-odd neutral scalar $H_{3}^{0}$.

\section{B.4 Decays to one scalar and one vector boson}

The decays of a scalar $H_{i}$ to a scalar $s$ and a vector boson $V$ are described by [166]

$$
\Gamma\left(H_{i} \rightarrow V s\right)=\frac{\left|C_{V H_{i} s^{*}}\right|^{2} m_{V}^{2}}{16 \pi m_{H_{i}}} \lambda\left(\frac{m_{H_{i}}^{2}}{m_{V}^{2}}, \frac{m_{s}^{2}}{m_{V}^{2}}\right) \lambda^{\frac{1}{2}}\left(\frac{m_{V}^{2}}{m_{H_{i}}^{2}}, \frac{m_{s}^{2}}{m_{H_{i}}^{2}}\right)
$$

where $\lambda(x, y)$ is defined by Eq. B.2. If the vector boson is off-shell, this becomes [166]

$$
\Gamma\left(H_{i} \rightarrow V^{*} s\right)=3 \delta_{V} \frac{\left|C_{V H_{i} s^{*}}\right|^{2} m_{V}^{2} m_{H_{i}}}{16 \pi^{3} v^{2}} G_{s V}
$$

Here we have $\delta_{W}=3 / 2, \delta_{Z}=3\left(7 / 12-10 / 9 s_{W}^{2}+40 / 27 s_{W}^{4}\right)$, and

$$
\begin{aligned}
G_{i j}= & \frac{1}{4}\left\{2\left(-1+k_{i}-k_{j}\right) \sqrt{\lambda_{i j}}\left[\frac{\pi}{2}+\arctan \left(\frac{k_{j}\left(1-k_{j}+k_{i}\right)-\lambda_{i j}}{\left(1-k_{i}\right) \sqrt{\lambda_{i j}}}\right)\right]\right. \\
& \left.\left.+\lambda_{i j}-2 k_{i}\right) \log k_{i}+\frac{1}{3}\left(1-k_{i}\right)\left[5\left(1+k_{i}\right)-4 k_{j}+\frac{2 \lambda_{i j}}{k_{j}}\right]\right\}
\end{aligned}
$$

where $k_{\ell}=m_{\ell}^{2} / m_{H_{i}}^{2}$ and $\lambda_{i j}=-1+2 k_{i}+2 k_{j}-\left(k_{i}-k_{j}\right)^{2}$. 


\section{B.5 Decays to fermions}

The decay of a scalar particle $H_{i}$ to fermions $\bar{f}_{1} f_{2}$ is described by [166]

$$
\Gamma\left(H_{i} \rightarrow \bar{f}_{1} f_{2}\right)=\frac{N_{c} m_{H}}{8 \pi}\left\{\left[1-\left(x_{1}+x_{2}\right)^{2}\right]\left|C^{S}\right|^{2}+\left[1-\left(x_{1}-x_{2}\right)^{2}\right]\left|C^{P}\right|^{2}\right\} \lambda^{\frac{1}{2}}\left(x_{1}^{2}, x_{2}^{2}\right)
$$

where $N_{c}=3$ for quarks (1 for leptons), $x_{1,2}=m_{f_{1,2}} / m_{H_{i}}$, and $\lambda(x, y)$ is defined by Eq. B.2. The scalar-fermion coupling is defined by $i\left(C^{S}+C^{P} \gamma_{5}\right)$, as in Appendix A.3.3. We do not implement QCD corrections in our results; a discussion of these corrections is available in Ref. [166]. 


\section{References}

[1] K. Hally, H. E. Logan, and T. Pilkington, "Constraints on large scalar multiplets from perturbative unitarity," Phys. Rev. D 85 (2012) 095017, [arXiv: 1202.5073].

[2] K. Earl, K. Hartling, H. E. Logan, and T. Pilkington, "Constraining models with a large scalar multiplet," Phys. Rev. D 88 (2013) 015002, [arXiv: 1303.1244].

[3] K. Earl, K. Hartling, H. E. Logan, and T. Pilkington, "Two viable large scalar multiplet models with a Z2 symmetry," Phys. Rev. D 90 (2014) 055029, [arXiv: 1311.3656].

[4] K. Hartling, K. Kumar, and H. E. Logan, "The decoupling limit in the Georgi-Machacek model," Phys. Rev. D 90 (2014) 015007, [arXiv: 1404.2640].

[5] M. Aoki and S. Kanemura, "Unitarity bounds in the Higgs model including triplet fields with custodial symmetry," Phys. Rev. D 77 (2008) 095009, [arXiv:0712.4053]. Erratum Phys. Rev. D 89, 059902 (2014).

[6] C.-W. Chiang, A.-L. Kuo, and K. Yagyu, "Enhancements of weak gauge boson scattering processes at the CERN LHC," JHEP 1310 (2013) 072, [arXiv: 1307.7526].

[7] K. Hartling, K. Kumar, and H. E. Logan, "Indirect constraints on the Georgi-Machacek model and implications for Higgs boson couplings," Phys. Rev. D 91 (2015) 015013, [arXiv: 1410.5538]. 
[8] G. Branco, P. Ferreira, L. Lavoura, M. Rebelo, M. Sher, et al., "Theory and phenomenology of Two-Higgs-Doublet models," Phys. Rept. 516 (2012) 1-102, [arXiv:1106.0034].

[9] S. P. Martin, "A supersymmetry primer," Adv. Ser. Direct. High Energy Phys. 21 (2010) 1-153, [hep-ph/9709356].

[10] J. Kalinowski, "SUSY theory review," Acta Physica Polonica B 38 (2007) 0531 .

[11] A. Djouadi, "Implications of the Higgs discovery for supersymmetry," EPJ Web Conf. 95 (2015) 03006.

[12] N. G. Deshpande and E. Ma, "Pattern of symmetry breaking with two Higgs doublets," Phys. Rev. D 18 (1978) 2574.

[13] C. Burgess, M. Pospelov, and T. ter Veldhuis, "The minimal model of nonbaryonic dark matter: a singlet scalar," Nucl. Phys. B 619 (2001) 709-728, [hep-ph/0011335].

[14] M. Cirelli, N. Fornengo, and A. Strumia, "Minimal dark matter," Nucl. Phys. B 753 (2006) 178-194, [hep-ph/0512090].

[15] M. Cirelli and A. Strumia, "Minimal Dark Matter: Model and results," New J. Phys. 11 (2009) 105005, [arXiv:0903.3381].

[16] Y. Cai, W. Chao, and S. Yang, "Scalar septuplet dark matter and enhanced $h \rightarrow \gamma \gamma$ decay rate," JHEP 1212 (2012) 043, [arXiv:1208.3949].

[17] F.-X. Josse-Michaux and E. Molinaro, "Triplet scalar dark matter and leptogenesis in an inverse seesaw model of neutrino mass generation," Phys. Rev. D 87 (2013) 036007, [arXiv:1210.7202].

[18] K. L. McDonald, "Minimal tree-level seesaws with a heavy intermediate fermion," JHEP 1307 (2013) 020, [arXiv: 1303.4573].

[19] K. Babu, S. Nandi, and Z. Tavartkiladze, "New mechanism for neutrino mass generation and triply charged Higgs bosons at the LHC," Phys. Rev. D 80 (2009) 071702, [arXiv:0905.2710].

[20] I. Picek and B. Radovcic, "Novel TeV-scale seesaw mechanism with Dirac mediators," Phys. Lett. B 687 (2010) 338-341, [arXiv:0911.1374]. 
[21] K. Kumericki, I. Picek, and B. Radovcic, "Exotic seesaw-motivated heavy leptons at the LHC," Phys. Rev. D 84 (2011) 093002, [arXiv:1106.1069].

[22] K. Kumericki, I. Picek, and B. Radovcic, "TeV-scale seesaw with quintuplet fermions," Phys. Rev. D 86 (2012) 013006, [arXiv:1204.6599].

[23] B. Ren, K. Tsumura, and X.-G. He, "A Higgs quadruplet for Type III Seesaw and implications for $\mu \rightarrow e \gamma$ and $\mu-e$ conversion," Phys. Rev. D 84 (2011) 073004, [arXiv:1107.5879].

[24] S. S. Law and K. L. McDonald, "Generalized inverse seesaw mechanisms," Phys. Rev. D 87 (2013) 113003, [arXiv:1303.4887].

[25] S. S. Law and K. L. McDonald, "A class of inert N-tuplet models with radiative neutrino mass and dark matter," JHEP 1309 (2013) 092, [arXiv: 1305.6467].

[26] C.-S. Chen, C.-Q. Geng, D. Huang, and L.-H. Tsai, "Many high-charged scalars in LHC searches and Majorana neutrino mass generations," Phys. Rev. D 87 (2013) 077702, [arXiv:1212.6208].

[27] K. L. McDonald, "Probing exotic fermions from a seesaw/radiative model at the LHC," JHEP 1311 (2013) 131, [arXiv:1310.0609].

[28] N. A. Ky and N. T. H. Van, "Scalar sextet in the 331 model with right-handed neutrinos," Phys. Rev. D 72 (2005) 115017, [hep-ph/0512096].

[29] M. Catano, R. Martinez, and F. Ochoa, "Neutrino masses in a 331 model with right-handed neutrinos without doubly charged Higgs bosons via inverse and double seesaw mechanisms," Phys. Rev. D 86 (2012) 073015, [arXiv: 1206.1966].

[30] H. Georgi and M. Machacek, "Doubly charged Higgs bosons," Nucl. Phys. B $262(1985) 463$.

[31] J. Hisano and K. Tsumura, "Higgs boson mixes with an SU(2) septet representation," Phys. Rev. D 87 (2013) 053004, [arXiv:1301.6455].

[32] S. Kanemura, M. Kikuchi, and K. Yagyu, "Probing exotic Higgs sectors from the precise measurement of Higgs boson couplings," Phys. Rev. D 88 (2013) 015020, [arXiv:1301.7303]. 
[33] C. Alvarado, L. Lehman, and B. Ostdiek, "Surveying the scope of the $S U(2)_{L}$ scalar septet sector," JHEP 1405 (2014) 150, [arXiv: 1404.3208].

[34] D. Zeppenfeld, R. Kinnunen, A. Nikitenko, and E. Richter-Was, "Measuring Higgs boson couplings at the CERN LHC," Phys. Rev. D 62 (2000) 013009, [hep-ph/0002036].

[35] P. Cushman, C. Galbiati, D. McKinsey, H. Robertson, T. Tait, et al., "Working Group Report: WIMP Dark Matter Direct Detection," arXiv: 1310.8327.

[36] H. E. Logan and V. Rentala, "All the generalized Georgi-Machacek models," arXiv: 1502.01275.

[37] S. Chang and J. G. Wacker, "Little Higgs and custodial SU(2)," Phys. Rev. D 69 (2004) 035002, [hep-ph/0303001].

[38] S. Chang, "A 'Littlest Higgs' model with custodial SU(2) symmetry," JHEP 0312 (2003) 057, [hep-ph/0306034].

[39] L. Cort, M. Garcia, and M. Quiros, "Supersymmetric custodial triplets," Phys. Rev. D 88 (2013) 075010, [arXiv: 1308.4025].

[40] M. Garcia-Pepin, S. Gori, M. Quiros, R. Vega, R. Vega-Morales, et al., "Supersymmetric custodial Higgs triplets and the breaking of universality," Phys. Rev. D 91 (2015) 015016, [arXiv:1409.5737].

[41] A. Delgado, M. Garcia-Pepin, and M. Quiros, "GMSB with Light Stops," arXiv:1505.07469.

[42] S. Godunov, M. Vysotsky, and E. Zhemchugov, "Double Higgs production at LHC, see-saw type II and Georgi-Machacek model," J. Exp. Theor. Phys. 120 (2015) 369-375, [arXiv: 1408.0184].

[43] Particle Data Group, K. Olive et al., "Review of particle physics," Chin. Phys. C 38 (2014) 090001.

[44] ATLAS and CMS Collaborations, G. Aad et al., "Combined measurement of the Higgs boson mass in $p p$ collisions at $\sqrt{s}=7$ and $8 \mathrm{TeV}$ with the ATLAS and CMS experiments," Phys. Rev. Lett. 114 (2015) 191803, [arXiv: 1503.07589]. 
[45] P. Langacker, "Structure of the Standard Model," hep-ph/0304186.

[46] S. Dawson, "Introduction to electroweak symmetry breaking," hep-ph/9901280.

[47] D. Griffiths, Introduction to elementary particles. Wiley-VCH, Weinheim, Germany, second ed., 2008.

[48] M. Peskin and D. Schroeder, An Introduction to Quantum Field Theory. Advanced book classics. Addison-Wesley Publishing Company, 1995.

[49] G. Marchesini, "QCD review," Int. J. Mod. Phys. A 22 (2007) 5443-5452, [hep-ph/0611115].

[50] J. Goldstone, "Field theories with superconductor solutions," Il Nuovo Cimento (1955-1965) 19 (1961) 154-164.

[51] J. Goldstone, A. Salam, and S. Weinberg, "Broken Symmetries," Phys. Rev. 127 (1962) 965-970.

[52] M. Schmaltz and D. Tucker-Smith, "Little Higgs review," Ann. Rev. Nucl. Part. Sci. 55 (2005) 229-270, [hep-ph/0502182].

[53] K. D. Lane, "An Introduction to technicolor," in Theoretical Advanced Study Institute (TASI 93) in Elementary Particle Physics: The Building Blocks of Creation - From Microfermius to Megaparsecs Boulder, Colorado, June 6-July 2, 1993, 1993. hep-ph/9401324.

[54] V. A. Miransky, M. Tanabashi, and K. Yamawaki, "Dynamical electroweak symmetry breaking with large anomalous dimension and t quark condensate," Physics Letters B 221 (1989), No. 2 177-183.

[55] C. T. Hill, "Topcolor: top quark condensation in a gauge extension of the standard model," Physics Letters B 266 (1991) 419-424.

[56] C. T. Hill, M. A. Luty, and E. A. Paschos, "Electroweak symmetry breaking by fourth-generation condensates and the neutrino spectrum," Phys. Rev. D 43 (May, 1991) 3011-3025.

[57] W. J. Marciano, "Exotic new quarks and dynamical symmetry breaking," Phys. Rev. D 21 (Apr, 1980) 2425-2428. 
[58] N. Arkani-Hamed, S. Dimopoulos, G. Dvali, and N. Kaloper, "Infinitely large new dimensions," Phys. Rev. Lett. 84 (2000) 586-589, [hep-th/9907209].

[59] L. Randall and R. Sundrum, "A large mass hierarchy from a small extra dimension," Phys. Rev. Lett. 83 (1999) 3370-3373, [hep-ph/9905221].

[60] L. Randall and R. Sundrum, "An alternative to compactification," Phys. Rev. Lett. 83 (1999) 4690-4693, [hep-th/9906064].

[61] O. Cremonesi, "Neutrino masses and neutrinoless double beta decay: status and expectations," arXiv:1002.1437.

[62] Snowmass 2013 Cosmic Frontier Working Groups 14, D. Bauer et al., "Dark Matter in the Coming Decade: Complementary Paths to Discovery and Beyond," Phys.Dark Univ. 7-8 (2013) 16-23, [arXiv: 1305.1605].

[63] S. Godunov, A. Rozanov, M. Vysotsky, and E. Zhemchugov, "Extending the Higgs sector: an extra singlet," arXiv:1503.01618.

[64] O. Fischer and J. J. van der Bij, "The Scalar Singlet-Triplet Dark Matter Model," JCAP 1401 (2014) 032, [arXiv:1311.1077].

[65] R. N. Mohapatra and G. Senjanovic, "Neutrino Mass and Spontaneous Parity Violation," Phys. Rev. Lett. 44 (1980) 912.

[66] R. N. Mohapatra and G. Senjanovic, "Neutrino Masses and Mixings in Gauge Models with Spontaneous Parity Violation," Phys. Rev. D23 (1981) 165.

[67] N. Arkani-Hamed, A. Cohen, E. Katz, and A. Nelson, "The Littlest Higgs," JHEP 0207 (2002) 034, [hep-ph/0206021].

[68] T. Araki, C. Geng, and K. I. Nagao, "Dark matter in Inert Triplet Models," Phys. Rev. D 83 (2011) 075014, [arXiv:1102.4906].

[69] A. Arhrib, R. Benbrik, M. Chabab, G. Moultaka, M. Peyranere, et al., "The Higgs potential in the Type II Seesaw Model," Phys. Rev. D 84 (2011) 095005, [arXiv:1105.1925].

[70] M. S. Chanowitz and M. Golden, "Higgs boson triplets with $M_{W}=M_{Z} \cos \theta_{W}, "$ Phys. Lett. B 165 (1985) 105. 
[71] ATLAS Collaboration, G. Aad et al., "Observation of a new particle in the search for the Standard Model Higgs boson with the ATLAS detector at the LHC," Phys. Lett. B716 (2012) 1-29, [arXiv:1207.7214].

[72] CMS, S. Chatrchyan et al., "Observation of a new boson at a mass of 125 GeV with the CMS experiment at the LHC," Phys. Lett. B716 (2012) 30-61, [arXiv: 1207.7235].

[73] CMS Collaboration, "Combination of Standard Model Higgs boson searches and measurements of the properties of the new boson with a mass near 125 GeV," CMS-PAS-HIG-13-005.

[74] ATLAS Collaboration, "Combined coupling measurements of the Higgs-like boson with the ATLAS detector using up to $25 \mathrm{fb}^{-1}$ of proton-proton collision data," ATLAS-CONF-2013-034.

[75] ATLAS Collaboration, "Measurements of the Higgs boson production and decay rates and coupling strengths using pp collision data at $\sqrt{s}=7$ and 8 $\mathrm{TeV}$ in the ATLAS experiment," ATLAS-CONF-2015-007.

[76] CMS Collaboration, V. Khachatryan et al., "Precise determination of the mass of the Higgs boson and tests of compatibility of its couplings with the standard model predictions using proton collisions at 7 and $8 \mathrm{TeV}$," Eur. Phys. J. C 75 (2015) 212, [arXiv:1412.8662].

[77] SLD Electroweak Group, DELPHI, ALEPH, SLD, SLD Heavy Flavour Group, OPAL, LEP Electroweak Working Group, L3, S. Schael et al., "Precision electroweak measurements on the $Z$ resonance," Phys. Rept. 427 (2006) 257-454, [hep-ex/0509008].

[78] ATLAS Collaboration, "Search for an invisibly decaying Higgs boson produced via vector boson fusion in $p p$ Collisions at $\sqrt{s}=8 \mathrm{TeV}$ using the ATLAS detector at the LHC," ATLAS-CONF-2015-004.

[79] S. Dittmaier, C. Mariotti, G. Passarino, R. Tanaka, et al., "Handbook of LHC Higgs Cross Sections: 2. Differential Distributions," arXiv:1201.3084.

[80] DELPHI Collaboration, M. Espirito Santo, K. Hultqvist, P. Johansson, and A. Lipniacka, "Search for neutralino pair production at s**(1/2) from 192-GeV to 208-GeV," DELPHI-2003-002. 
[81] OPAL Collaboration, G. Abbiendi et al., "Search for chargino and neutralino production at $\mathrm{s}^{* *}(1 / 2)=192 \mathrm{GeV}$ to $209 \mathrm{GeV}$ at LEP," Eur. Phys. J. C35 (2004) 1-20, [hep-ex/0401026].

[82] DELPHI, OPAL, ALEPH, LEP Working Group for Higgs Boson Searches, L3, S. Schael et al., "Search for neutral MSSM Higgs bosons at LEP," Eur. Phys. J. C47 (2006) 547-587, [hep-ex/0602042].

[83] ALEPH, DELPHI, L3, OPAL, and LEP Collaborations, G. Abbiendi et al., "Search for Charged Higgs bosons: Combined Results Using LEP Data," Eur.Phys.J. C73 (2013) 2463, [arXiv:1301.6065].

[84] M. E. Peskin and T. Takeuchi, "A new constraint on a strongly interacting Higgs sector," Phys. Rev. Lett. 65 (1990) 964-967.

[85] M. E. Peskin and T. Takeuchi, "Estimation of oblique electroweak corrections," Phys. Rev. D 46 (1992) 381-409.

[86] I. Maksymyk, C. P. Burgess, and D. London, "Beyond S, T and U," Phys. Rev. D50 (1994) 529-535, [hep-ph/9306267].

[87] C. P. Burgess, S. Godfrey, H. Konig, D. London, and I. Maksymyk, "A Global fit to extended oblique parameters," Phys. Lett. B326 (1994) 276-281, [hep-ph/9307337].

[88] L. Lavoura and L.-F. Li, "Making the small oblique parameters large," Phys. Rev. D 49 (1994) 1409-1416, [hep-ph/9309262].

[89] Gfitter Group, M. Baak et al., "The global electroweak fit at NNLO and prospects for the LHC and ILC," Eur. Phys. J. C 74 (2014) 3046, [arXiv: 1407.3792].

[90] CMS Collaboration, V. Khachatryan et al., "Observation of the diphoton decay of the Higgs boson and measurement of its properties," Eur. Phys. J. C 74 (2014) 3076, [arXiv: 1407.0558].

[91] CMS Collaboration, S. Chatrchyan et al., "Search for a Higgs boson decaying into a $\mathrm{Z}$ and a photon in pp collisions at $\sqrt{s}=7$ and $8 \mathrm{TeV}$," Phys. Lett. B 726 (2013) 587-609, [arXiv:1307.5515].

[92] J. Gunion, H. Haber, G. Kane, and S. Dawson, Higgs Hunters Guide. Addison-Wesley Publishing Company, 1990. 
[93] LHC Higgs Cross Section Working Group, A. David et al., "LHC HXSWG interim recommendations to explore the coupling structure of a Higgs-like particle," arXiv:1209.0040.

[94] H. E. Haber and H. E. Logan, "Radiative corrections to the $Z b \bar{b}$ vertex and constraints on extended Higgs sectors," Phys. Rev. D 62 (2000) 015011, [hep-ph/9909335].

[95] U. Haisch and S. Westhoff, "Massive color-octet bosons: bounds on effects in top-quark pair production," JHEP 1108 (2011) 088, [arXiv:1106.0529].

[96] H. Baer, T. Barklow, K. Fujii, Y. Gao, A. Hoang, et al., "The International Linear Collider Technical Design Report - Volume 2: Physics," arXiv:1306. 6352.

[97] A. Wahab El Kaffas, P. Osland, and O. M. Ogreid, "Constraining the Two-Higgs-Doublet-Model parameter space," Phys. Rev. D 76 (2007) 095001, [arXiv:0706.2997].

[98] F. Mahmoudi and O. Stal, "Flavor constraints on the Two-Higgs-Doublet model with general Yukawa couplings," Phys. Rev. D 81 (2010) 035016, [arXiv:0907.1791].

[99] T. Inami and C. Lim, "Effects of superheavy quarks and leptons in low-energy weak processes $K_{L} \rightarrow \mu \bar{\mu}, K^{+} \rightarrow \pi^{+} \nu \bar{\nu}$ and $K_{0} \rightarrow \bar{K}_{0}$," Prog. Theor. Phys. 65 (1981) 297. Erratum: Prog. Theor. Phys. 65, 1772 (1981).

[100] A. Lenz, U. Nierste, J. Charles, S. Descotes-Genon, H. Lacker, et al., "Constraints on new physics in $B-\bar{B}$ mixing in the light of recent $\mathrm{LHCb}$ data," Phys. Rev. D 86 (2012) 033008, [arXiv:1203.0238].

[101] CKMfitter results are summarized at http://ckmfitter.in2p3.fr. The Rfit method used by CKMfitter is described in J. Charles et al. [CKMfitter Group Collaboration], Eur. Phys. J. C 41, 1 (2005) [hep-ph/0406184].

[102] U. Nierste, "B mixing in the Standard Model and beyond," arXiv:1212.5805.

[103] C. Bouchard, E. Freeland, C. Bernard, A. El-Khadra, E. Gamiz, et al., "Neutral $B$ mixing from $2+1$ flavor lattice-QCD: the Standard Model and beyond," PoS LATTICE2011 (2011) 274, [arXiv:1112.5642]. 
[104] A. Lenz and U. Nierste, "Numerical updates of lifetimes and mixing parameters of B mesons," arXiv:1102.4274.

[105] X.-Q. Li, J. Lu, and A. Pich, " $B_{s, d}^{0} \rightarrow \ell^{+} \ell^{-}$decays in the Aligned Two-Higgs-Doublet Model," JHEP 1406 (2014) 022, [arXiv:1404.5865].

[106] CMS and LHCb Collaborations, "Combination of results on the rare decays $B_{(s)}^{0} \rightarrow \mu^{+} \mu^{-}$from the CMS and LHCb experiments," CMS-PAS-BPH-13-007, LHCb-CONF-2013-012, CERN-LHCb-CONF-2013-012.

[107] C. Bobeth, M. Gorbahn, T. Hermann, M. Misiak, E. Stamou, et al., " $B_{s, d}->l+l-$ in the Standard Model with reduced theoretical uncertainty," Phys. Rev. Lett. 112 (2014) 101801, [arXiv:1311.0903].

[108] R. Aaij et al., "Implications of LHCb measurements and future prospects," Eur. Phys. J. C 73 (2013) 2373, [arXiv: 1208.3355].

[109] A. J. Buras, A. Kwiatkowski, and N. Pott, "On the scale uncertainties in the $B \rightarrow X(s) \gamma$ decay," Phys. Lett. B 414 (1997) 157-165, [hep-ph/9707482].

[110] D. Asner et al., "Averages of $b$-hadron, $c$-hadron, and $\tau$-lepton properties," arXiv: 1010.1589.

[111] J. Lees et al., "Measurement of $\mathrm{B}\left(B \rightarrow X_{s} \gamma\right)$, the $B \rightarrow X_{s} \gamma$ photon energy spectrum, and the direct CP asymmetry in $B \rightarrow X_{s+d} \gamma$ decays," Phys. Rev. D 86 (2012) 112008, [arXiv:1207.5772].

[112] M. Misiak, H. Asatrian, K. Bieri, M. Czakon, A. Czarnecki, et al., "Estimate of $\operatorname{BR}(\bar{B} \rightarrow X(s) \gamma)$ at $\mathrm{O}\left(\alpha(s)^{2}\right)$," Phys. Rev. Lett. 98 (2007) 022002, [hep-ph/0609232].

[113] T. Becher and M. Neubert, "Analysis of $\operatorname{Br}(\bar{B} \rightarrow X(s) \gamma)$ at NNLO with a cut on photon energy," Phys. Rev. Lett. 98 (2007) 022003, [hep-ph/0610067].

[114] T. Aushev, W. Bartel, A. Bondar, J. Brodzicka, T. Browder, et al., "Physics at Super B Factory," arXiv:1002.5012.

[115] G. Steigman, B. Dasgupta, and J. F. Beacom, "Precise relic WIMP abundance and its impact on searches for dark matter annihilation," Phys. Rev. D 86 (2012) 023506, [arXiv: 1204.3622].

[116] DMTools website, http://dmtools.brown.edu. 
[117] XENON1T Collaboration, E. Aprile, "The XENON1T dark matter search experiment," Springer Proc. Phys. 12-02-22 (2013) 93-96, [arXiv: 1206.6288].

[118] XENON100 Collaboration, E. Aprile et al., "Dark matter results from 225 live days of XENON100 data," Phys. Rev. Lett. 109 (2012) 181301, [arXiv: 1207.5988].

[119] LUX Collaboration, D. Akerib et al., "The Large Underground Xenon (LUX) Experiment," Nucl. Instrum. Meth. A 704 (2013) 111-126, [arXiv: 1211.3788].

[120] LUX Collaboration, D. Akerib et al., "First results from the LUX dark matter experiment at the Sanford Underground Research Facility," Phys. Rev. Lett. 112 (2014) 091303, [arXiv:1310.8214].

[121] DEAP Collaboration, M. Boulay, "DEAP-3600 dark matter search at SNOLAB," J. Phys. Conf. Ser. 375 (2012) 012027, [arXiv: 1203.0604].

[122] P. A. Amaudruz, M. Batygov, B. Beltran, J. Bonatt, M. Boulay, et al., "DEAP-3600 Dark Matter Search," arXiv:1410.7673.

[123] J. Cooley, "Overview of non-liquid noble direct detection dark matter experiments," Phys. Dark Univ. 4 (2014) 92-97, [arXiv:1410.4960].

[124] M. Baak, M. Goebel, J. Haller, A. Hoecker, D. Kennedy, et al., "The electroweak fit of the Standard Model after the discovery of a new boson at the LHC," Eur. Phys. J. C 72 (2012) 2205, [arXiv:1209.2716].

[125] J. Kopp, E. T. Neil, R. Primulando, and J. Zupan, "From gamma ray line signals of dark matter to the LHC," Phys. Dark Univ. 2 (2013) 22-34, [arXiv: 1301.1683].

[126] ATLAS Collaboration, "Measurements of the properties of the Higgs-like boson in the two photon decay channel with the ATLAS detector using $25 \mathrm{fb}^{1}$ of proton-proton collision data," ATLAS-CONF-2013-012.

[127] CMS Collaboration, "Updated measurements of the Higgs boson at $125 \mathrm{GeV}$ in the two photon decay channel," CMS-PAS-HIG-13-001. 
[128] S. S. AbdusSalam and T. A. Chowdhury, "Scalar representations in the light of electroweak phase transition and cold dark matter phenomenology," JCAP 1405 (2014) 026, [arXiv:1310.8152].

[129] H. E. Logan, T. A. Martin, and T. Pilkington, "LHC constraints on large scalar multiplet models with a $Z_{2}$ symmetry," arXiv:1507.01887.

[130] J. R. Ellis, A. Ferstl, and K. A. Olive, "Reevaluation of the elastic scattering of supersymmetric dark matter," Phys. Lett. B 481 (2000) 304-314, [hep-ph/0001005].

[131] J. Hisano, K. Ishiwata, N. Nagata, and T. Takesako, "Direct detection of electroweak-interacting dark matter," JHEP 1107 (2011) 005, [arXiv: 1104.0228].

[132] J. Gunion, R. Vega, and J. Wudka, "Higgs triplets in the Standard Model," Phys.Rev. D42 (1990) 1673-1691.

[133] J. Gunion, R. Vega, and J. Wudka, "Naturalness problems for rho $=1$ and other large one loop effects for a Standard Model Higgs sector containing triplet fields," Phys. Rev. D 43 (1991) 2322-2336.

[134] A. Akeroyd, "Cascade decays of triplet Higgs bosons at LEP-2," Phys.Lett. B442 (1998) 335-343, [hep-ph/9807409].

[135] S. Godfrey and K. Moats, "Exploring Higgs triplet models via vector boson scattering at the LHC," Phys. Rev. D 81 (2010) 075026, [arXiv: 1003.3033].

[136] D. Carmi, A. Falkowski, E. Kuflik, T. Volansky, and J. Zupan, "Higgs after the discovery: a status report," JHEP 1210 (2012) 196, [arXiv:1207.1718].

[137] S. Chang, C. A. Newby, N. Raj, and C. Wanotayaroj, "Revisiting Theories with Enhanced Higgs Couplings to Weak Gauge Bosons," Phys. Rev. D 86 (2012) 095015, [arXiv:1207.0493].

[138] C. Englert, E. Re, and M. Spannowsky, "Triplet Higgs boson collider phenomenology after the LHC," Phys. Rev. D 87 (2013) 095014, [arXiv: 1302.6505].

[139] C. Englert, E. Re, and M. Spannowsky, "Pinning down Higgs triplets at the LHC," Phys. Rev. D 88 (2013) 035024, [arXiv:1306.6228]. 
[140] A. Efrati and Y. Nir, "What if $\lambda_{h h h} \neq 3 m_{h}^{2} / v$," arXiv:1401.0935.

[141] G. Belanger, B. Dumont, U. Ellwanger, J. Gunion, and S. Kraml, "Global fit to Higgs signal strengths and couplings and implications for extended Higgs sectors," Phys. Rev. D 88 (2013) 075008, [arXiv: 1306.2941].

[142] I. Low and J. Lykken, "Revealing the electroweak properties of a new scalar resonance," JHEP 10 (2010) 053, [arXiv: 1005.0872].

[143] H. E. Logan and M.-A. Roy, "Higgs couplings in a model with triplets," Phys. Rev. D82 (2010) 115011, [arXiv: 1008.4869].

[144] C.-W. Chiang and K. Yagyu, "Testing the custodial symmetry in the Higgs sector of the Georgi-Machacek model," JHEP 1301 (2013) 026, [arXiv: 1211.2658].

[145] C.-W. Chiang and T. Yamada, "Electroweak phase transition in Georgi-Machacek model," Phys. Lett. B 735 (2014) 295-300, [arXiv: 1404.5182].

[146] C.-W. Chiang, S. Kanemura, and K. Yagyu, "Novel constraint on the parameter space of the Georgi-Machacek model with current LHC data," Phys. Rev. D 90 (2014) 115025, [arXiv: 1407.5053].

[147] R. Killick, K. Kumar, and H. E. Logan, "Learning what the Higgs boson is mixed with," Phys. Rev. D 88 (2013) 033015, [arXiv:1305.7236].

[148] C.-W. Chiang and K. Tsumura, "Properties and searches of the exotic neutral Higgs bosons in the Georgi-Machacek model," JHEP 1504 (2015) 113, [arXiv: 1501.04257 .

[149] J. F. Gunion and H. E. Haber, "The CP-conserving Two Higgs Doublet Model: the approach to the decoupling limit," Phys. Rev. D 67 (2003) 075019, [hep-ph/0207010].

[150] ATLAS, CDF, CMS, and D0 Collaborations, "First combination of Tevatron and LHC measurements of the top-quark mass," arXiv:1403.4427.

[151] K. Nakamura et al., "Review of particle physics," J.Phys. G37 (2010) 075021. 
[152] V. D. Barger, J. Hewett, and R. Phillips, "New Constraints on the Charged Higgs Sector in Two Higgs Doublet Models," Phys. Rev. D 41 (1990) 3421-3441.

[153] M. Ciuchini, G. Degrassi, P. Gambino, and G. Giudice, "Next-to-leading QCD corrections to $\bar{B} \rightarrow X(s) \gamma$ : Standard Model and Two Higgs Doublet Model," Nucl. Phys. B 527 (1998) 21-43, [hep-ph/9710335].

[154] F. Mahmoudi, "SuperIso: a program for calculating the isospin asymmetry of $B \rightarrow K \gamma$ in the MSSM," Comput. Phys. Commun. 178 (2008) 745-754, [arXiv:0710.2067].

[155] F. Mahmoudi, "SuperIso v2.3: a program for calculating flavor physics observables in Supersymmetry," Comput. Phys. Commun. 180 (2009) 1579-1613, [arXiv:0808.3144].

[156] F. Mahmoudi, "Superiso v3.0, flavor physics observables calculations: extension to \{NMSSM\}," Comput. Phys. Commun. 180 (2009), No. 91718 1719.

[157] M. Misiak and M. Steinhauser, "NNLO QCD corrections to the $\bar{B} \rightarrow X(s) \gamma$ matrix elements using interpolation in m(c)," Nucl. Phys. B 764 (2007) 62-82, [hep-ph/0609241].

[158] D. Eriksson, J. Rathsman, and O. Stal, "2HDMC: Two-Higgs-Doublet Model Calculator physics and manual," Comput. Phys. Commun. 181 (2010) 189-205, [arXiv:0902.0851].

[159] F. Mahmoudi, private communication.

[160] G. G. Athanasiu, P. J. Franzini, and F. J. Gilman, "Restrictions on two Higgs models from heavy quark systems," Phys. Rev. D 32 (1985) 3010-3019.

[161] L. Abbott, P. Sikivie, and M. B. Wise, "Constraints on charged Higgs couplings," Phys. Rev. D 21 (1980) 1393.

[162] A. Lenz, "Theoretical update of B-Mixing and Lifetimes," arXiv:1205.1444.

[163] A. Pich and P. Tuzon, "Yukawa alignment in the Two-Higgs-Doublet Model," Phys. Rev. D 80 (2009) 091702, [arXiv:0908.1554]. 
[164] A. Denner, R. Guth, W. Hollik, and J. H. Kuhn, "The Z width in the Two Higgs Doublet Model," Z. Phys. C 51 (1991) 695-705.

[165] A. K. Grant, "The heavy top quark in the Two Higgs Doublet Model," Phys. Rev. D 51 (1995) 207-217, [hep-ph/9410267].

[166] K. Hartling, K. Kumar, and H. E. Logan, "GMCALC: a calculator for the Georgi-Machacek model," arXiv:1412.7387.

[167] M. Duhrssen, S. Heinemeyer, H. Logan, D. Rainwater, G. Weiglein, et al., "Extracting Higgs boson couplings from CERN LHC data," Phys. Rev. D 70 (2004) 113009, [hep-ph/0406323].

[168] F. Caola and K. Melnikov, "Constraining the Higgs boson width with ZZ production at the LHC," Phys. Rev. D 88 (2013) 054024, [arXiv:1307.4935].

[169] CMS Collaboration, "Constraints on the Higgs boson width from off-shell production and decay to ZZ to $\ell \ell \ell$ and $\ell \ell \nu \nu, "$ CMS-PAS-HIG-14-002.

[170] C. Englert and M. Spannowsky, "Limitations and opportunities of off-shell coupling measurements," Phys. Rev. D 90 (2014) 053003, [arXiv:1405.0285].

[171] ATLAS, G. Aad et al., "Search for anomalous production of prompt same-sign lepton pairs and pair-produced doubly charged Higgs bosons with $\sqrt{s}=8 \mathrm{TeV}$ pp collisions using the ATLAS detector," JHEP 03 (2015) 041, [arXiv: 1412.0237].

[172] ATLAS Collaboration, "Evidence for electroweak production of $W^{ \pm} W^{ \pm} j j$ in $p p$ collisions at $\sqrt{s}=8 \mathrm{TeV}$ with the ATLAS detector," ATLAS-CONF-2014-013.

[173] ATLAS Collaboration, G. Aad et al., "Search for a charged Higgs boson produced in the vector-boson fusion mode with decay $H^{ \pm} \rightarrow W^{ \pm} Z$ using $p p$ collisions at $\sqrt{s}=8 \mathrm{TeV}$ with the ATLAS experiment," Phys. Rev. Lett. 114 (2015) 231801, [arXiv: 1503.04233].

[174] ATLAS Collaboration, G. Aad et al., "Search for a CP-odd Higgs boson decaying to $\mathrm{Zh}$ in pp collisions at $\sqrt{s}=8 \mathrm{TeV}$ with the ATLAS detector," Phys. Lett. B 744 (2015) 163-183, [arXiv:1502.04478].

[175] V. Ilisie and A. Pich, "Low-mass fermiophobic charged Higgs phenomenology in Two-Higgs-Doublet models," JHEP 1409 (2014) 089, [arXiv: 1405.6639]. 
[176] J. C. Romao and S. Andringa, "Vector boson decays of the Higgs boson," Eur. Phys. J. 77 (1999) 631-642, [hep-ph/9807536].

[177] R. Contino, M. Ghezzi, C. Grojean, M. Mhlleitner, and M. Spira, "eHDECAY: an implementation of the Higgs Effective Lagrangian into HDECAY," Comput. Phys. Commun. 185 (2014) 3412-3423, [arXiv:1403.3381]. 